\title{
Energiebilanz bei Forstwirten
}

\section{Diskrepanz zwischen Energieumsatz und Nährstoffaufnahme} bei unterschiedlichen Anforderungssituationen

\author{
Dissertation \\ zur Erlangung des Doktorgrades \\ der Sozialwissenschaftlichen Fakultät \\ der Georg-August-Universität Göttingen
}

vorgelegt von

Stefanie Gramkow

geboren in Stade

Göttingen, 2015 
Betreuungsausschuss

Erstbetreuer: $\quad$ Prof. Dr. Dr. A. Niklas

Weitere Betreuer: Prof. Dr. A. Krüger

Prof. Dr. Dr. H.-W. Buhmann

Tag der mündlichen Prüfung: 03. November 2015 


\section{$\underline{\text { Inhaltsverzeichnis }}$}

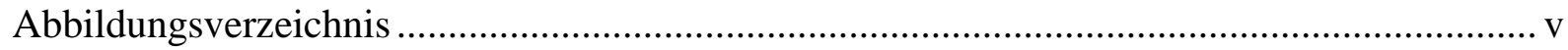

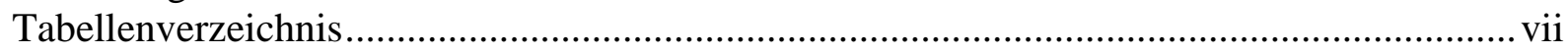

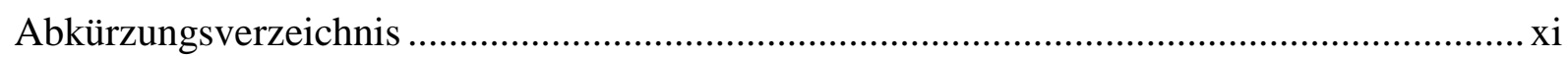

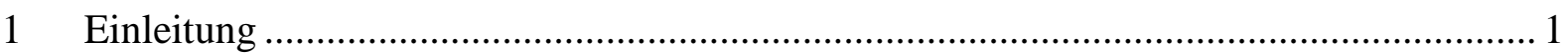

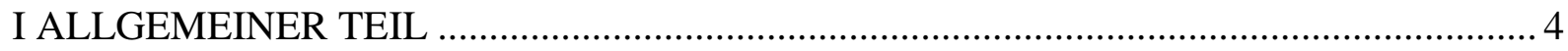

2 Energieaufnahme

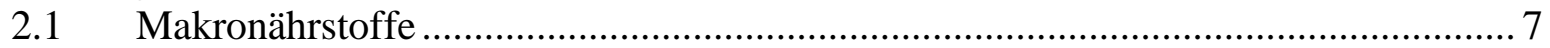

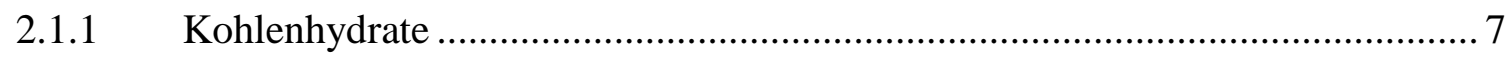

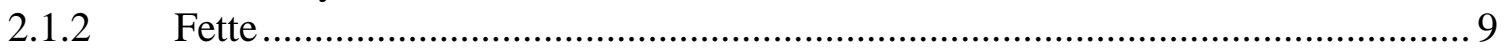

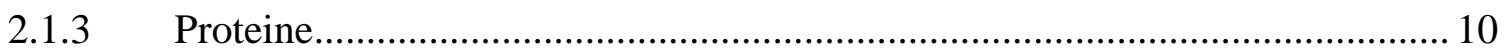

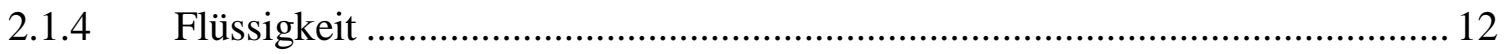

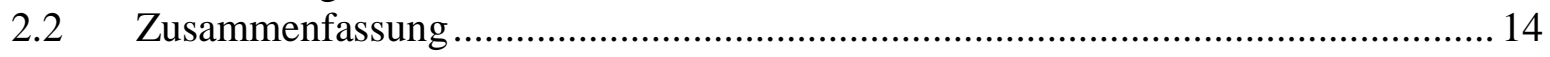

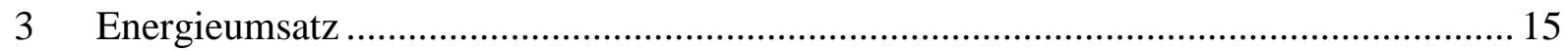

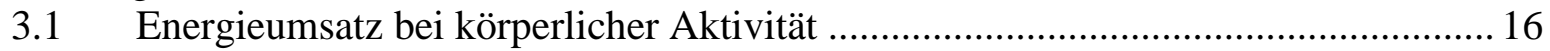

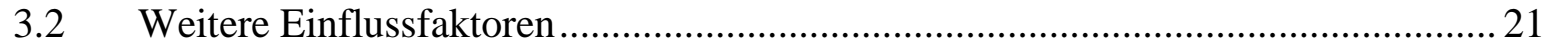

3.3 Wirkungsgrad und Respiratorischer Quotient ....................................................... 21

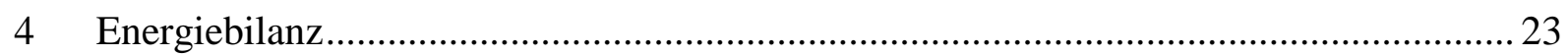

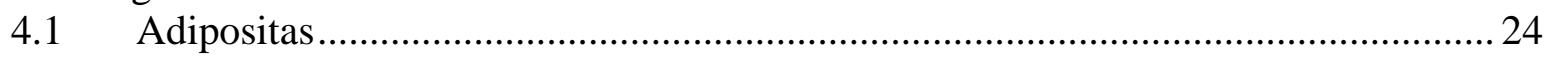

4.2 Klassifizierung von Adipositas: Body Mass Index vs. Körperfettanteil .................. 26

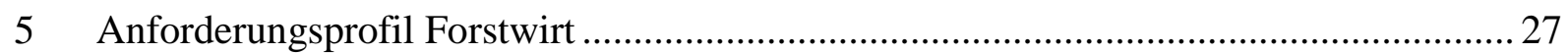

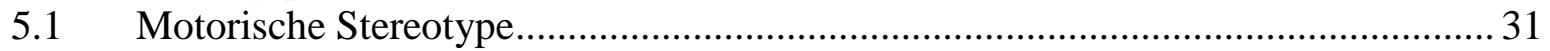

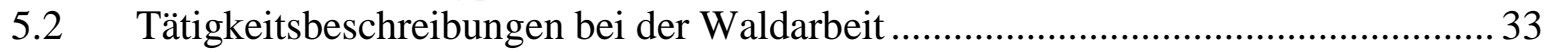

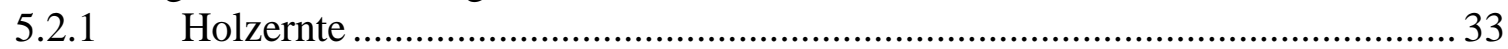

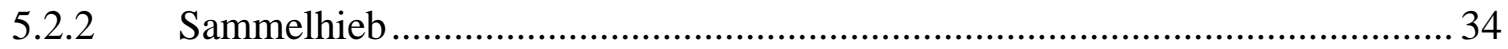

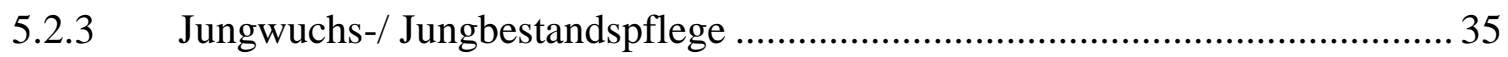

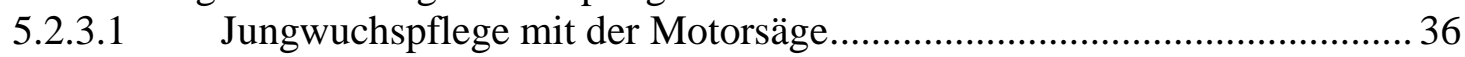

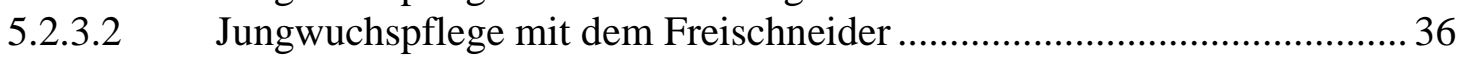

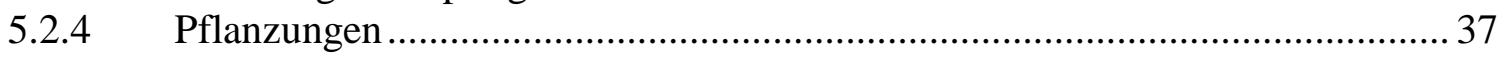

5.2.5 Bau von Geländern ............................................................................. 40

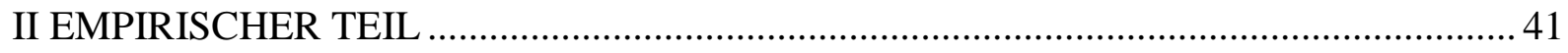

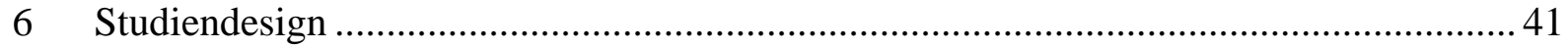

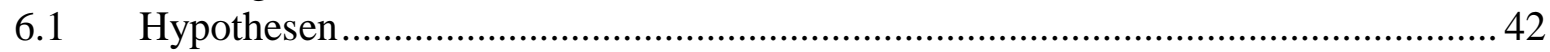

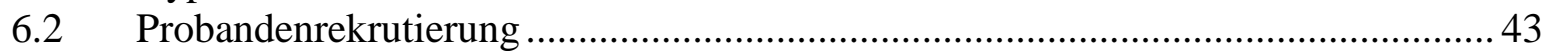

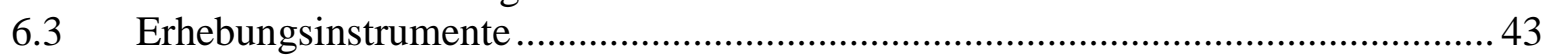

6.3.1 Soziodemografische und anthropometrische Daten ................................... 44

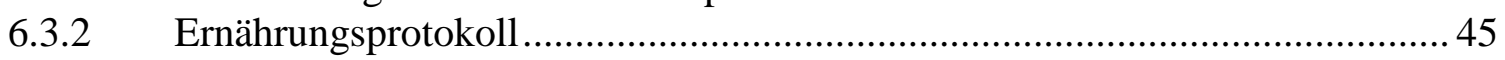

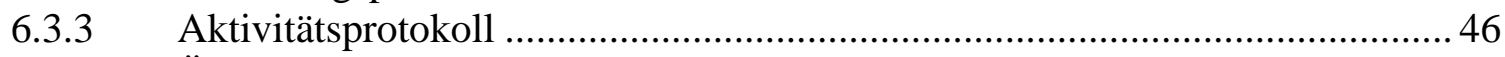

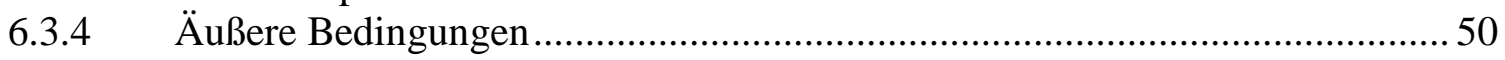

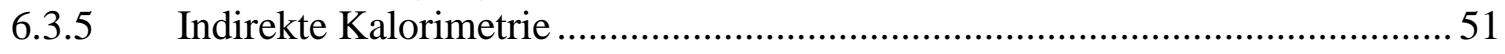




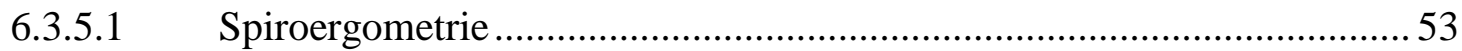

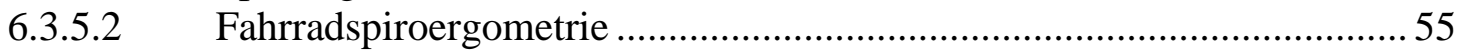

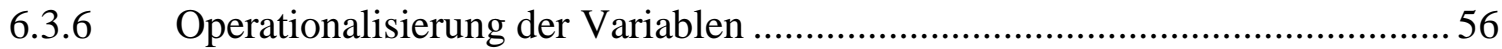

6.3.7 Statistische Auswertung der Ergebnisse ........................................................... 58

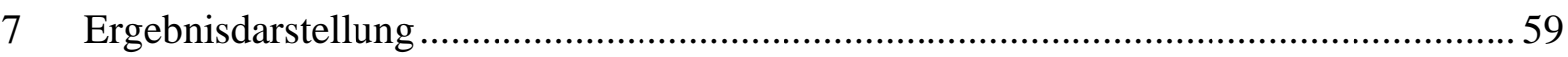

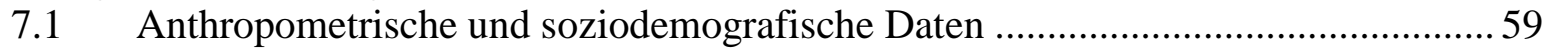

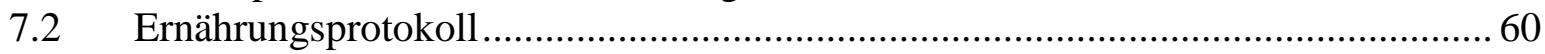

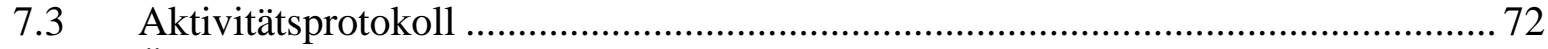

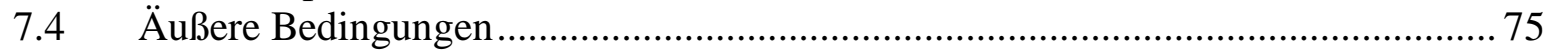

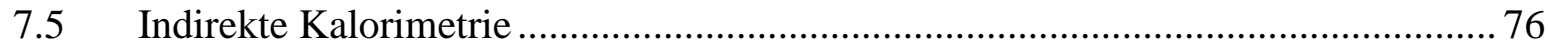

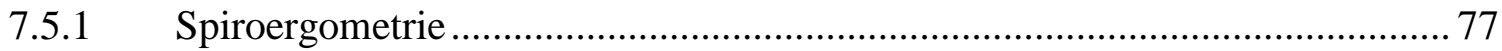

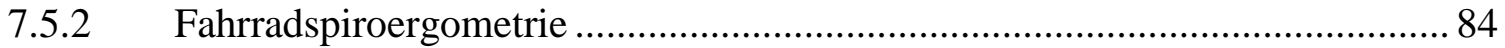

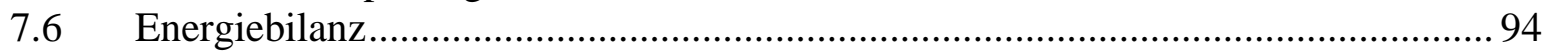

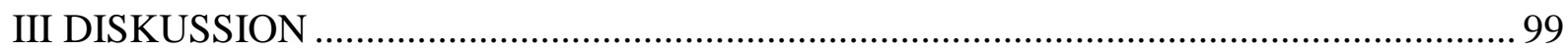

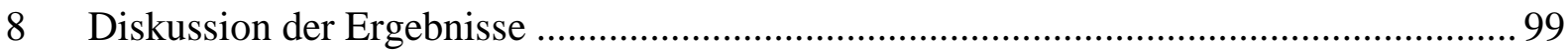

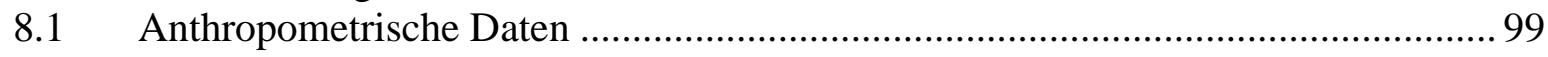

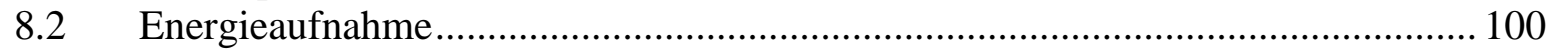

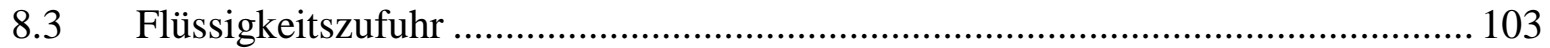

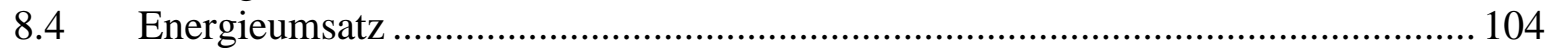

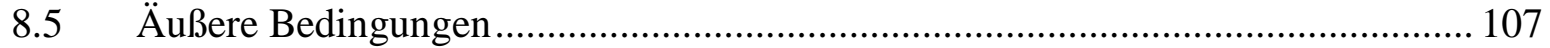

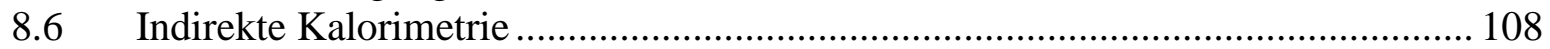

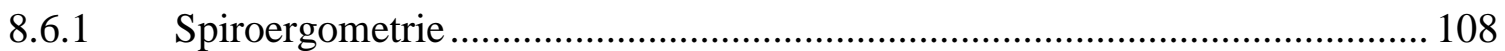

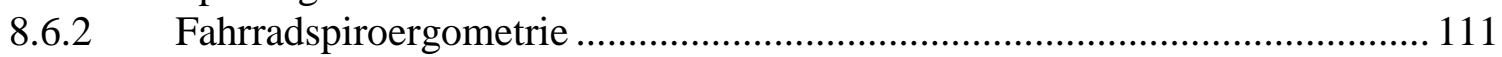

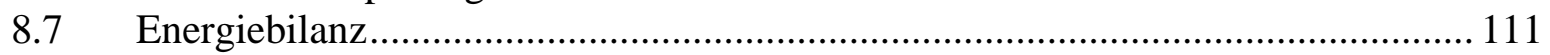

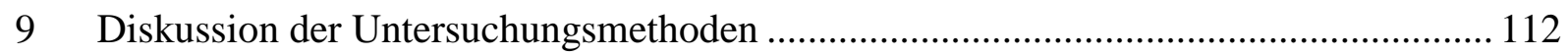

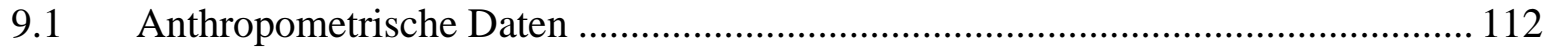

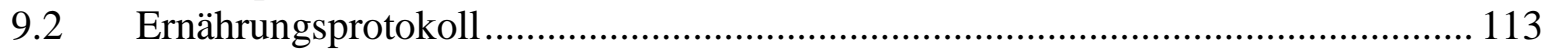

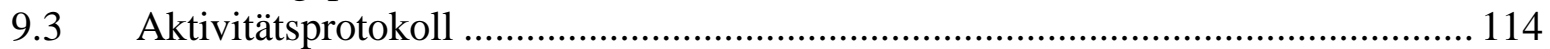

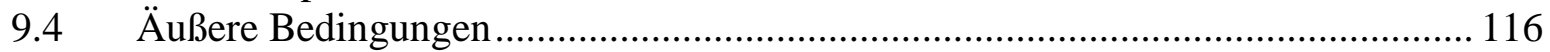

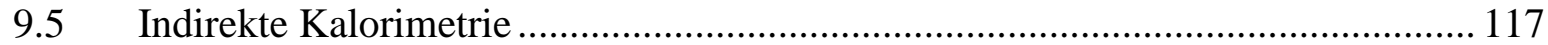

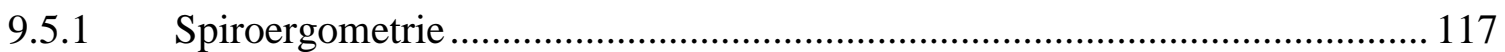

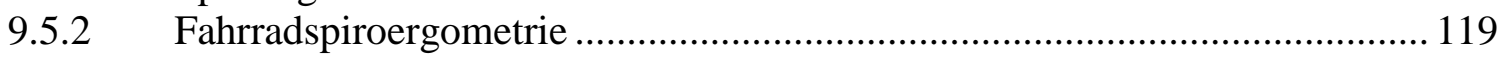

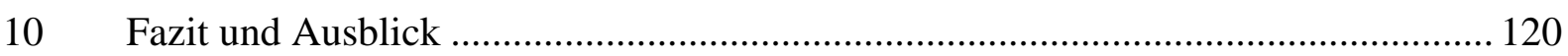

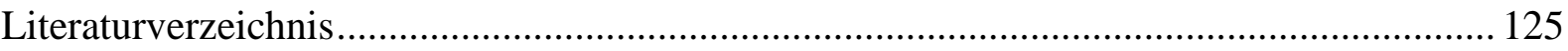




\section{Abbildungsverzeichnis}

Abbildung 1: Aufteilung des täglichen Energiebedarfs bei leichter, mittelschwerer, schwerer u. schwerster Arbeit mit Angaben der Dauerleistungsgrenze.

Abbildung 2: Forstwirt bei motormanueller Holzernte........................................................ 34

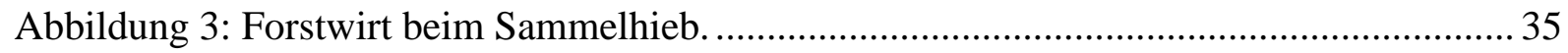

Abbildung 4: Forstwirt bei der Jungwuchspflege mit der leichten Motorsäge. ....................... 36

Abbildung 5: Forstwirt bei der Jungwuchspflege mit dem Freischneider. ............................ 37

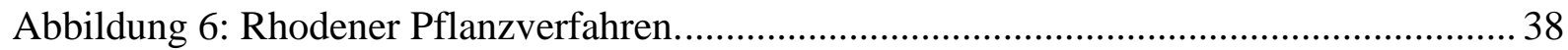

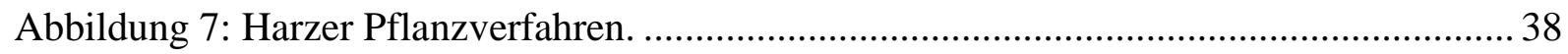

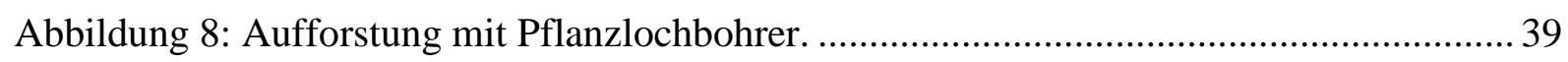

Abbildung 9: Forstwirte beim Gießen eines Fundaments................................................. 40

Abbildung 10: Forstwirt mit angelegtem Spiroergometrie-System und speziellem

Gesichtsschutz.

Abbildung 11: Empfohlene Ernährungszusammensetzung und Zusammensetzung der Ernährung in t1.

Abbildung 12: Empfohlene Ernährungszusammensetzung und Zusammensetzung der Ernährung in t2.

Abbildung 13: Empfohlene Ernährungszusammensetzung und Zusammensetzung der Ernährung in $\mathrm{t} 3$

Abbildung 14: Zivilisationskost und durchschnittliche Ernährungsanteile in der Stichprobe. 66

Abbildung 15: Durchschnittlicher Anteil von Kohlenhydraten, Fett und Eiweiß in t1 pro Person (in \%).

Abbildung 16: Durchschnittlicher Anteil von Kohlenhydraten, Fett und Eiweiß in t2 pro Person (in \%).

Abbildung 17: Durchschnittlicher Anteil von Kohlenhydraten, Fett und Eiweiß in t3 pro Person (in \%)

Abbildung 18: Sauerstoffaufnahme in $1 /$ min bei Spiroergometrie. .79

Abbildung 19: Relativer Energieumsatz in kcal/ kg KG/ min bei Spiroergometrie. 80

Abbildung 20: RQ bei Spiroergometrie. 80 
Abbildung 21: Relativer Energieumsatz FW01 bei Spiroergometrie und

Fahrradspiroergometrie (FE)

Abbildung 22: Relativer Energieumsatz FW03 bei Spiroergometrie und

Fahrradspiroergometrie (FE)

Abbildung 23: Relativer Energieumsatz FW04 bei Spiroergometrie und

Fahrradspiroergometrie (FE).

Abbildung 24: Relativer Energieumsatz FW05 bei Spiroergometrie und

Fahrradspiroergometrie (FE)

Abbildung 25: Sauerstoffaufnahme FW01 bei Spiroergometrie und Fahrradspiroergometrie

(FE).

Abbildung 26: Sauerstoffaufnahme FW03 bei Spiroergometrie und Fahrradspiroergometrie (FE)

Abbildung 27: Sauerstoffaufnahme FW04 bei Spiroergometrie und Fahrradspiroergometrie $(\mathrm{FE})$

Abbildung 28: Sauerstoffaufnahme FW05 bei Spiroergometrie und Fahrradspiroergometrie (FE)

Abbildung 29: Energiebilanz in kcal 


\section{Tabellenverzeichnis}

Tabelle 1: Exemplarische Energieumsätze.

Tabelle 2: Arbeitsenergieumsatz ( $\mathrm{kcal} / \mathrm{min}$ ) bei beruflichen Tätigkeiten in der

Forstwirtschaft.

Tabelle 3: Produktive Arbeitsstunden bei der Waldarbeit.

Tabelle 4: MET-Werte für körperliche Aktivitäten bei Forstwirten.

Tabelle 5: Allgemeine Zeit und Reine Arbeitszeit für forstwirtschaftliche Tätigkeiten.

Tabelle 6: Kategorisierung der Hangneigung.

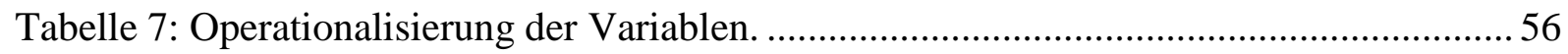

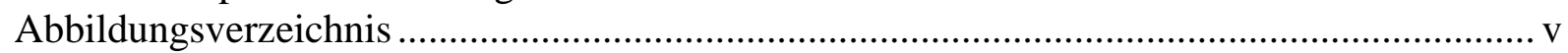

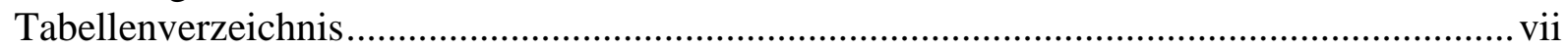

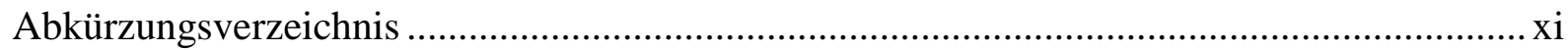

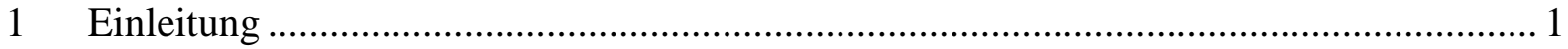

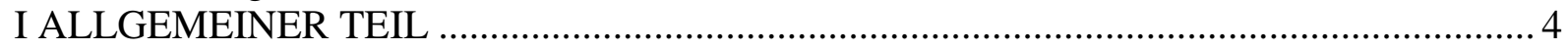

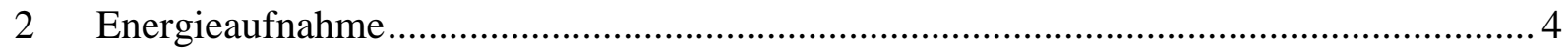

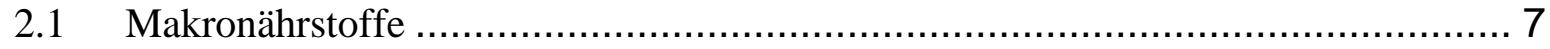

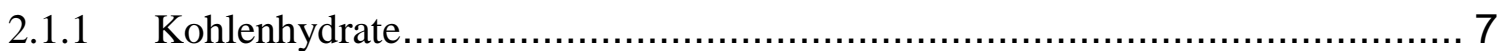

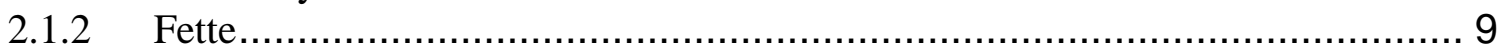

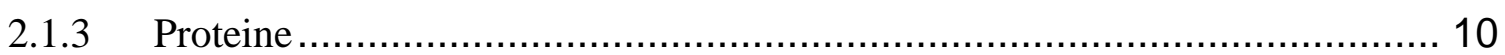

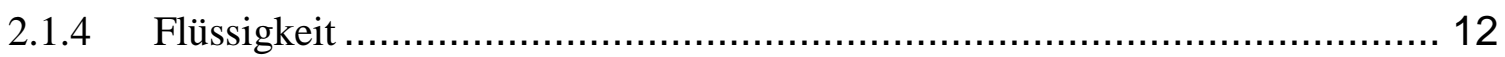

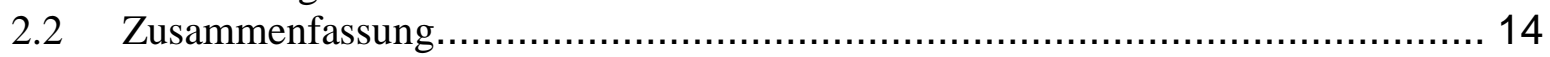

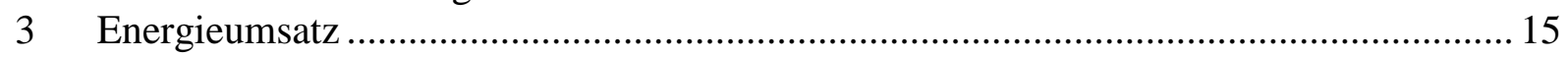

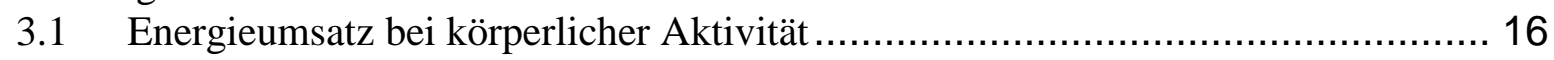

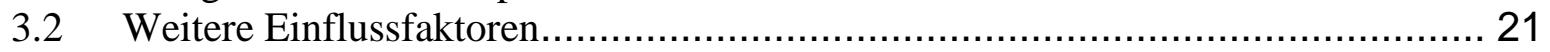

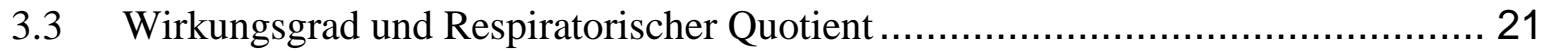

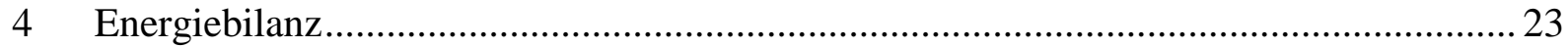

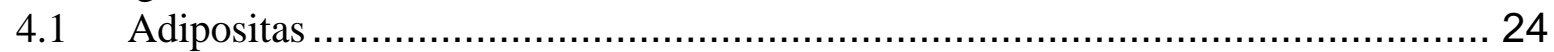

4.2 Klassifizierung von Adipositas: Body Mass Index vs. Körperfettanteil ................ 26

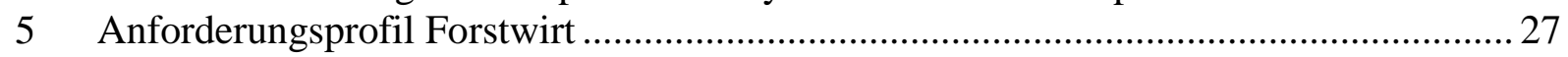

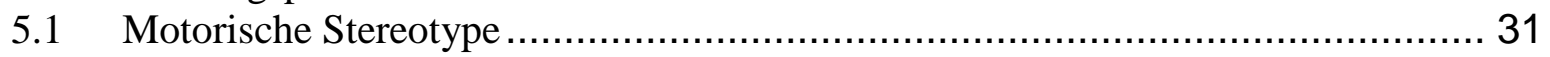

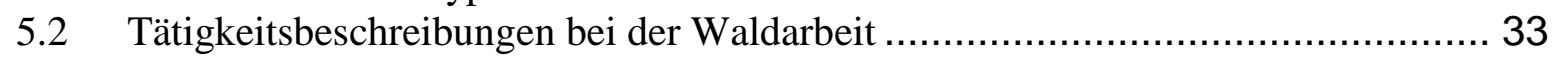

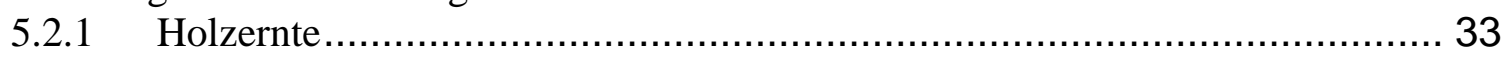

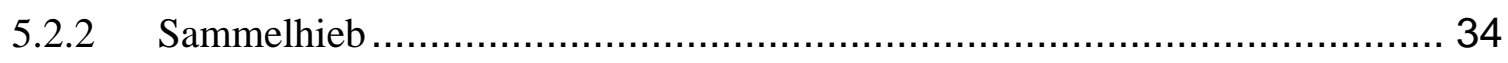

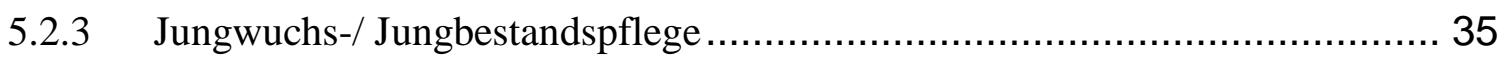

5.2.3.1 Jungwuchspflege mit der Motorsäge ........................................ 36

5.2.3.2 Jungwuchspflege mit dem Freischneider ................................. 36

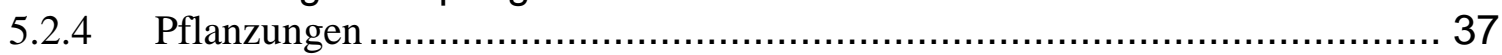

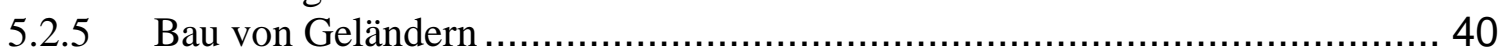

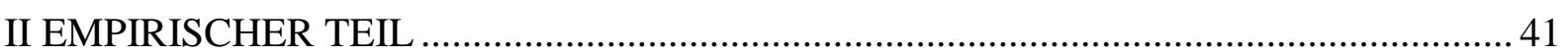

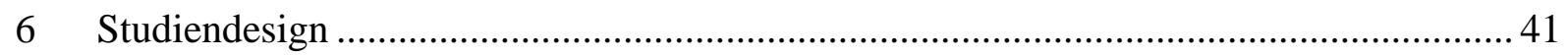

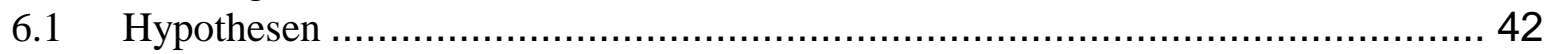

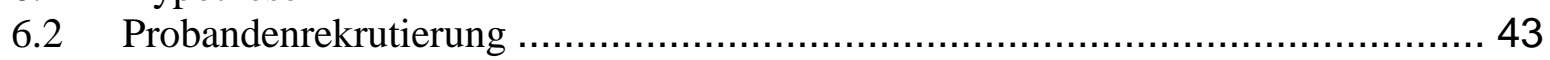

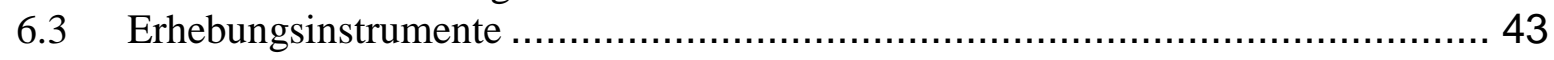


6.3.1 Soziodemografische und anthropometrische Daten ........................... 44

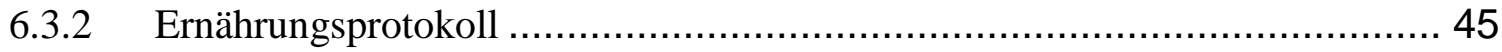

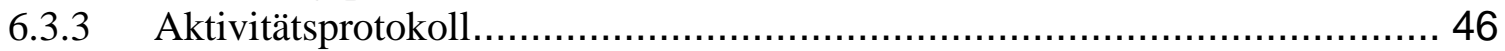

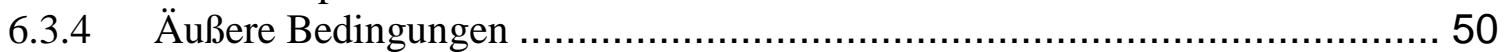

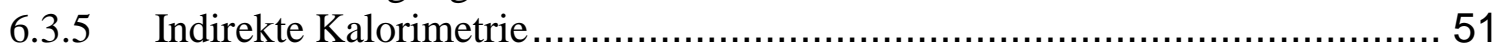

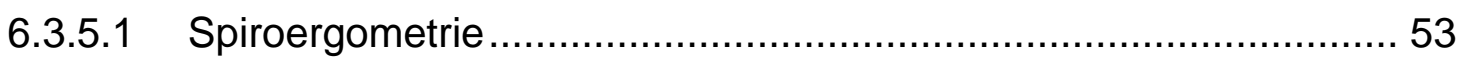

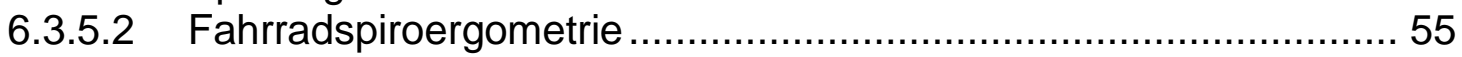

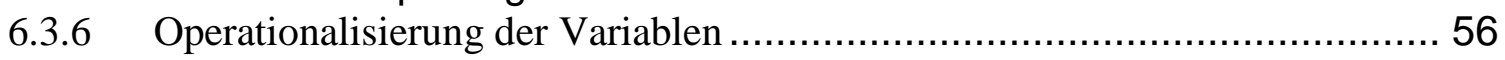

6.3.7 Statistische Auswertung der Ergebnisse ........................................ 58

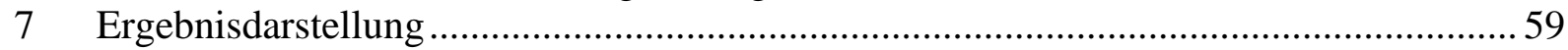

7.1 Anthropometrische und soziodemografische Daten.................................. 59

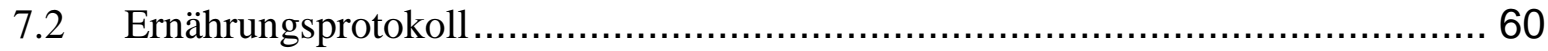

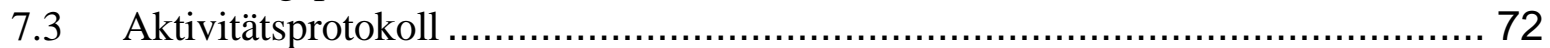

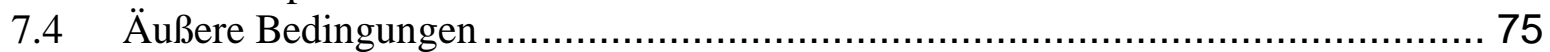

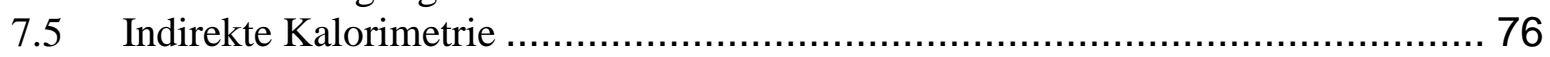

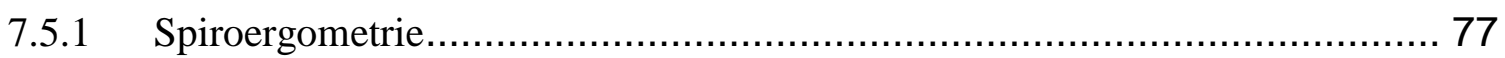

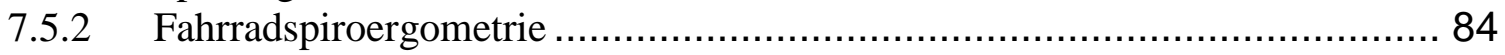

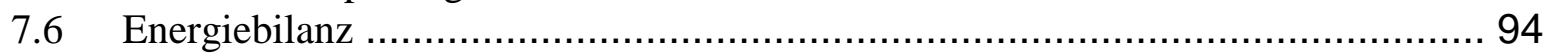

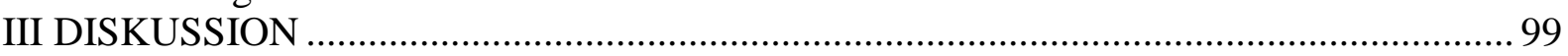

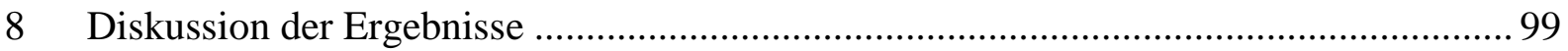

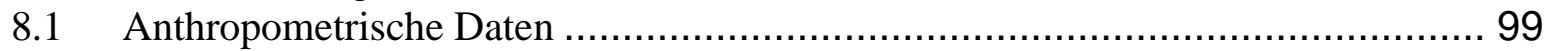

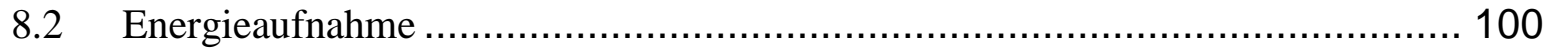

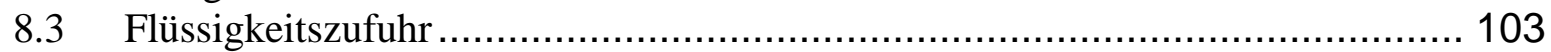

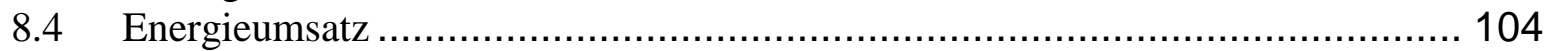

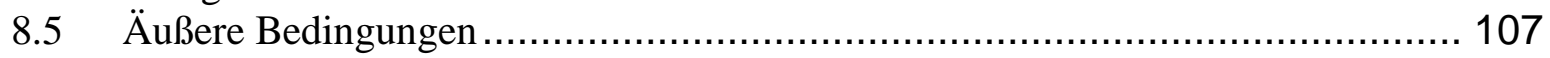

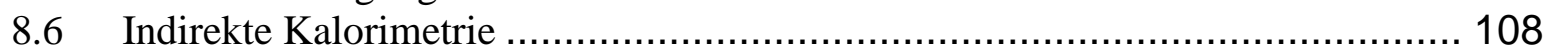

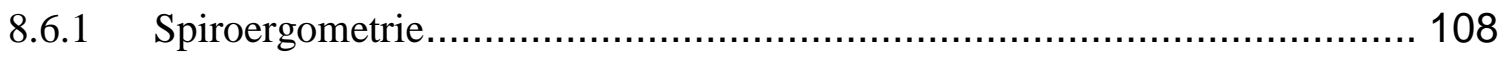

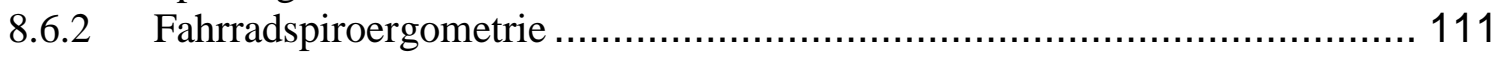

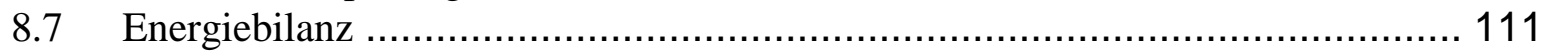

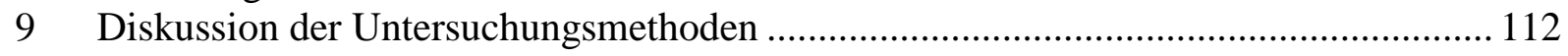

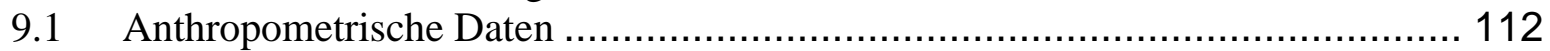

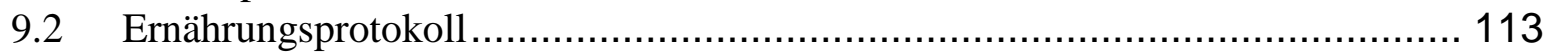

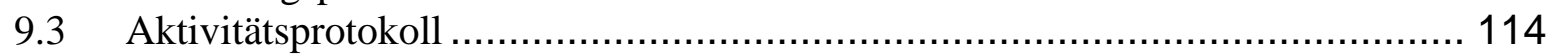

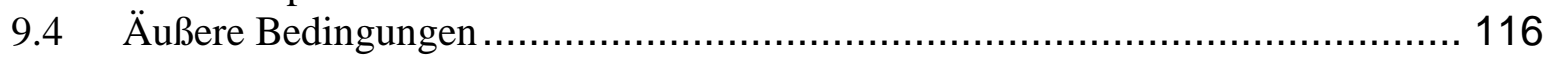

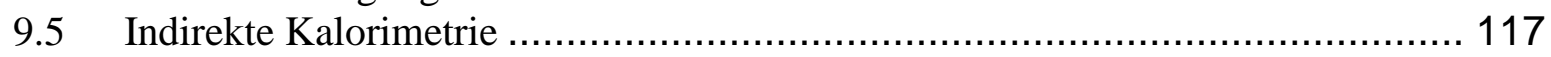

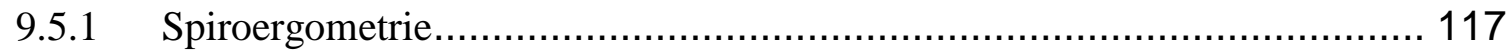

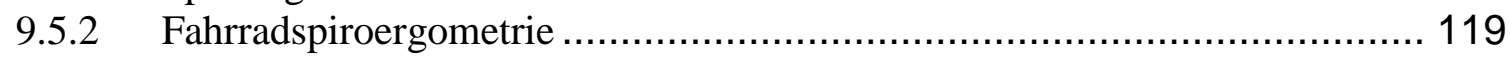

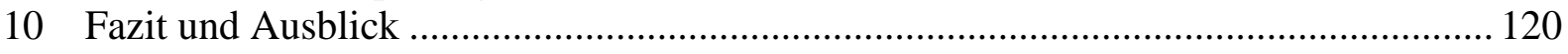

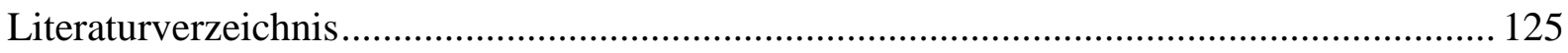

Tabelle 8: Häufigkeitstabelle zu den Items Alter, Größe, Gewicht und BMI......................... 60

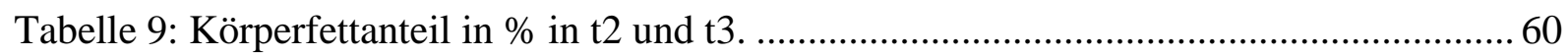

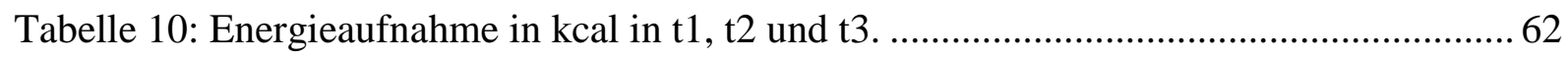

Tabelle 11: Vergleich der durchschnittlichen Energieaufnahmen zu verschiedenen

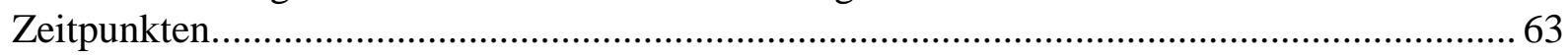

Tabelle 12: Überblick über die Zusammensetzung der Ernährung (in \%)..............................66 
Tabelle 13: Auflistung der prozentualen Anteile von Kohlenhydraten, Fett und Eiweiß in t1t3.

Tabelle 14: Wasser- (in 1) und Alkoholkonsum (in g) im Durchschnitt. ................................. 71

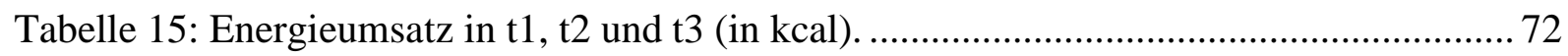

Tabelle 16: Energieumsatz in der Freizeit in t1, t2 und $\mathrm{t} 3$ (in kcal).................................... 73

Tabelle 17: Energieumsatz durch sportliche Aktivität (in kcal). ........................................ 73

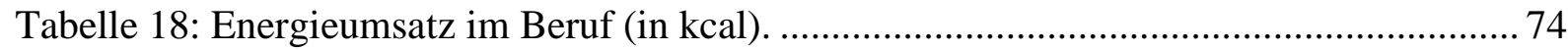

Tabelle 19: Deskriptive Statistik der äußeren Bedingungen................................................... 75

Tabelle 20: Korrelation Energieumsatz und Sauerstoffverbrauch - äußere Bedingungen. ..... 76

Tabelle 21: Kalorische Äquivalente für die Umrechnung des Energieumsatzes. .................... 77

Tabelle 22: Deskriptive Statistik Spiroergometrie ............................................................ 78

Tabelle 23: Paarvergleich Sauerstoffaufnahme (Spiroergometrie)....................................... 81

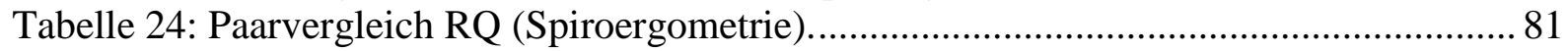

Tabelle 25: Paarvergleich relativer Energieumsatz (Spiroergometrie) ................................ 81

Tabelle 26: Zuordnung von Energieumsatz und Tätigkeiten der Forstwirte. .......................... 82

Tabelle 27: MET der einzelnen gemessenen Tätigkeiten. .................................................. 82

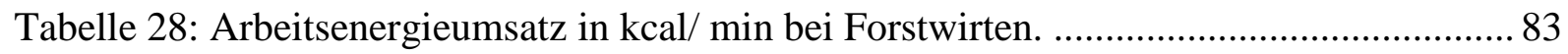

Tabelle 29: Gemessene RMR und errechnete RMR und BMR (in kcal/ min). ......................83

Tabelle 30: Leistungen in Watt der Forstwirte bei der Fahrradspiroergometrie...................... 84

Tabelle 31: Relativer Energieumsatz bei Fahrradspiroergometrie in kcal/ kg KG/ min......... 85

Tabelle 32: Zugeordnete Leistung in Watt zum relativen Energieumsatz und zur

Sauerstoffaufnahme.

Tabelle 33: Zuordnung des relativen Energieumsatzes (in kcal/ kg KG/ min) bei beruflicher

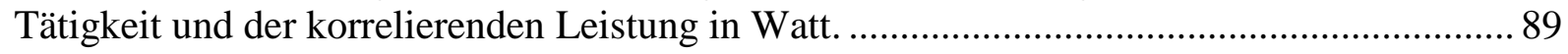

Tabelle 34: Sauerstoffaufnahme bei Fahrradspiroergometrie............................................ 90

Tabelle 35: Sauerstoffaufnahme bei beruflicher Tätigkeit und korrelierende Leistungen in Watt. 
Tabelle 37: T-Test zwischen den Mittelwerten der Energiebilanzen in t1, t2 und t3. .96

Tabelle 38: Korrelationen zwischen der Energiebilanz und dem Energieumsatz...................97 


\section{Abkürzungsverzeichnis}

\begin{tabular}{ll} 
AZ & Allgemeine Zeit \\
BHD & Brusthöhendurchmesser \\
BMI & Body Mass Index \\
bzw. & beziehungsweise \\
C & Celsius \\
ca. & circa \\
d & Tag \\
ebd. & ebenda \\
et al. & et alii/ et aliae (= und andere) \\
g & Gramm \\
kcal & Kilokalorie \\
kJ & Kilojoule \\
kg & Kilogramm \\
l & Liter \\
MET & Metabolic Equivalent of Task \\
ml & Milliliter \\
o. g. & oben genannt \\
PAL & Physical Activity Level \\
RAZ & Reine Arbeitszeit \\
RQ & Respiratorischer Quotient \\
s. o. & siehe oben \\
u. a. & unter anderem \\
u. U. & unter Umständen \\
v. a. & vor allem \\
vgl. & vergleiche \\
W & Watt \\
WHO & World Health Organisation \\
z. B. & zum Beispiel \\
z. T. & zum Teil \\
& \\
\hline &
\end{tabular}




\section{$1 \quad$ Einleitung}

„Bewegungsmangel gilt heute als größtes Gesundheitsrisiko“ (Persson 2007). Zahlreiche Untersuchungen zu diesem Thema bestätigen die Relevanz der körperlichen Aktivität für die Gesundheit. Durch die zunehmende Mechanisierung der Arbeitsplätze und die zunehmende Anzahl der sitzenden Tätigkeiten im Beruf sinkt die Alltagsaktivität. Eine ungünstige Ernährung, wie sie häufig in der Zivilisationskost vorliegt, stellt mit der körperlichen Inaktivität „Risikoverhaltensweisen dar, welche langfristig die Gesundheit beeinträchtigen können“ (Niermann 2011).

Vor diesem Hintergrund hat sich in den letzten Jahren ein Forschungsschwerpunkt herausgebildet, der sich mit dem Bewegungsmangel in der Bevölkerung und seinen Folgen beschäftigt. Einen weiteren Forschungsschwerpunkt bildet die Ernährung. Dabei konnten Einflüsse der Ernährung auf unterschiedliche Faktoren wie z. B. allgemeiner Gesundheitszustand, sportliche Leistungsfähigkeit und Produktivität am Arbeitsplatz aufgezeigt werden (Stone 1994a; Çalişkan \& Çağlar 2010). Konsequenz dieser Forschungsergebnisse ist es, Interventionen und Verhaltensweisen zu fördern, die sich der Problematik des Bewegungsmangels annehmen und körperliche Aktivität und bedarfsangepasste Ernährung thematisieren. Ziel solcher Interventionen ist eine Steigerung des Energieumsatzes durch körperliche Freizeitaktivität und durch die Umgestaltung der Arbeitsplätze für mehr Bewegung (Saris et al. 2003; Berg \& König 2005; Straker \& Mathiassen 2009). Konsequenz solcher Interventionen ist eine ausgewogene oder negative Energiebilanz, um u. a. ein konstantes oder sinkendes Körpergewicht zu erreichen.

Grundlage für diese Analyse und die daraus folgende Konzipierung der Interventionen bildet aufgrund der starken Verbreitung die Bevölkerungsgruppe mit Bewegungsmangel. Folglich geraten bei der Forschung Berufsgruppen in den Hintergrund, die einer hohen körperlichen Belastung ausgesetzt sind. Wenn auch der allgemeine Trend in den Industrieländern zu vermindertem Energieverbrauch geht (Wirth 2003; Straker \& Mathiassen 2009), so bestehen trotz fortschreitender Mechanisierung weiterhin Berufsgruppen mit hoher körperlicher Beanspruchung, zu denen u. a. die Berufsgruppe der Forstwirte zählt. Aus der hohen körperlichen Aktivität im Beruf darf nicht fälschlicherweise die Schlussfolgerung gezogen werden, dass hieraus automatisch eine gesündere Lebensweise resultiert. Durch die meist statische und einseitige Belastung bewirkt die berufliche körperliche Aktivität bei Schwerarbeitern keine positiven gesundheitlichen Auswirkungen (Hollmann et al. 2006). 
In der Berufsgruppe der Forstwirte konnte Rudolph (2013) nachweisen, dass eine bewegungsbezogene Intervention bei körperlich aktiven Arbeitern notwendig ist, um physische und psychosoziale Aspekte aufzugreifen und zu verbessern. U. a. konnte ein saisonales Belastungsprofil erkannt werden, das Belastungsspitzen mit entsprechenden Auswirkungen auf motorische Beanspruchungsformen einschließt.

Die in der Studie erhobenen Messdaten umfassen u. a. Körpergewicht und Körpergröße der Probanden, um den Body Mass Index (= BMI) berechnen zu können. Die Probanden wiegen im Durchschnitt 94,5 kg bei einer durchschnittlichen Größe von 1,79 m. Dies entspricht einem BMI von 29,4. Dieser Wert ist in den leicht übergewichtigen Bereich einzuordnen, der die Werte 26 bis 30 einschließt, so dass eine Gewichtsreduzierung empfohlen wird (Geiss \& Hamm 1992). Neben dem Aspekt, dass eine ausgewogene Ernährung zur Erhaltung der Gesundheit und der Leistungsfähigkeit führt (Sluik et al. 2008), ist ein erhöhter BMI mit steigenden Gesundheitskosten verbunden (Clark et al. 2015). Trotz der hohen körperlichen Aktivität im Beruf und einem vermutlich hohen Energieumsatz liegt bei den Forstwirten ein Übergewicht vor. Da das Anforderungsprofil der Forstwirte eine hohe körperliche Beanspruchung umfasst, ist von einem erhöhten Energiebedarf auszugehen. Es stellt sich somit die Frage, ob das saisonale Belastungsprofil neben den Auswirkungen auf das muskuloskelettale System auch Auswirkungen auf die Energiebilanz der Forstwirte hat.

Das Ziel der vorliegenden Arbeit ist eine Analyse der Energiebilanz bei Forstwirten. Das bei Rudolph (2013) erhobene Übergewicht der Forstwirte steht im Widerspruch zu deren hohen körperlichen Aktivität im Beruf. Durch die hohe Beanspruchung lässt sich ein hoher Energieverbrauch vermuten. Um trotz dieses hohen Energieverbrauchs eine positive Energiebilanz und somit eine Gewichtszunahme zu erreichen, müsste die Energieaufnahme über dem Energieverbrauch liegen.

Um die Energiebilanz zu untersuchen, ist eine Gegenüberstellung von Energieumsatz durch körperliche Aktivität und Energieaufnahme durch die Ernährung notwendig. Berücksichtigung finden unterschiedliche Parameter, z. B. die verschiedenen beruflichen Tätigkeiten der Forstwirte zu unterschiedlichen Jahreszeiten und die äußeren Einflüsse auf die körperliche Aktivität. Aktuelle Werte des Energieumsatzes bei den beruflichen Tätigkeiten werden mittels indirekter Kalorimetrie erhoben, so dass sie mit anderen bereits erhobenen Werten vergleichbar sind. Durch die Datenerhebung soll neben der Energiebilanz der Forstwirte geprüft werden, ob sich die saisonalen Belastungsprofile auch auf den Energieumsatz auswirken. Des Weiteren wird der Frage nachgegangen, ob es bei unterschiedlicher körperlicher Aktivität zu einer Anpassung der Ernährung an den Energieumsatz kommt. Die Erhebung der Körper- 
komposition ermöglicht eine Kontrolle über mögliche Auswirkungen der Energiebilanzen. Grundlegend werden über einen Zeitraum von einem dreiviertel Jahr in drei Messzeitpunkten verschiedene Daten erhoben. Im Fokus der Untersuchung steht die Betrachtung, wie sich die Energiebilanz bei Forstwirten zu unterschiedlichen Jahreszeiten und somit unterschiedlichen beruflichen Tätigkeiten gestaltet.

Die Arbeit gliedert sich in drei Hauptteile. Im ersten Teil werden die theoretischen Grundlagen auf dem aktuellen Stand der Forschung dargelegt. Nach der Darstellung von Energieaufnahme (Kapitel 2) und Energieumsatz (Kapitel 3) werden diese beiden Themen in Kapitel 4 zur Energiebilanz miteinander verknüpft, wobei speziell auf Adipositas als Folge einer positiven Energiebilanz eingegangen wird. Mit der Beschreibung und Analyse des Anforderungsprofils Forstwirt (Kapitel 5) schließt der allgemeine Teil der Arbeit ab, so dass die Ergebnisse der Untersuchung auf die forstwirtspezifische Beanspruchung rückbezogen werden können.

Der empirische und somit zweite Teil der Arbeit beginnt mit der Hypothesenformulierung, die auf der theoretischen Grundlage erfolgt (Kapitel 6). Nach der Darstellung der Erhebungsinstrumente und der Methoden werden die Ergebnisse der einzelnen Untersuchungen dargestellt (Kapitel 7). Die Hypothesenüberprüfung findet integrativ in der Ergebnisdarstellung statt.

Eine ausführliche Diskussion unter Einbezug des aktuellen Forschungsstands findet im dritten Teil der Arbeit statt. Hierbei werden neben der Diskussion der Ergebnisse (Kapitel 8) auch Grenzen und Möglichkeiten der Untersuchungsmethoden diskutiert (Kapitel 9). Den Abschluss dieser Arbeit bilden das Fazit und ein Ausblick (Kapitel 10). 


\section{ALLGEMEINER TEIL}

Im ersten Teil der Arbeit werden der theoretische Hintergrund und der aktuelle Forschungsstand dargelegt. Neben einer allgemeinen Abhandlung der Themen wird auch im Speziellen Bezug auf das Berufsbild Forstwirt genommen. Nachdem zunächst die Themen Energieaufnahme (Kapitel 2) und Energieumsatz (Kapitel 3) betrachtet werden, werden diese anschlieBend im Abschnitt zur Energiebilanz zueinander in Beziehung gesetzt. Um die Energiebilanz bei Forstwirten analysieren zu können, endet der allgemeine Teil mit einer Betrachtung des beruflichen Anforderungsprofils der Forstwirte.

\section{Energieaufnahme}

Die lebensnotwendige Energie gewinnt der menschliche Organismus aus der Nahrung über das Verdauungssystem. Der für die Verarbeitung notwendige Sauerstoff wird über das HerzLungen-Gefäßsystem aufgenommen. Mittels der Verdauungsprozesse sowie der Umbau- und Aufbauprozesse werden den Nahrungsmitteln die wichtigen Nahrungsstoffe entnommen und den Stoffwechselprozessen in den jeweiligen Körperzellen zugeführt. Energie wird durch die Oxidation von Kohlenhydraten, Fetten und Proteinen gewonnen. Der Wirkungsgrad dieses Vorgangs beträgt nie $100 \%$, da der Stoffwechsel neben der mechanischen Energie Wärme erzeugt (Atzler 1927; Åstrand 1987). Der äußere Stoffwechsel bezeichnet hierbei die Vorgänge der Aufnahme oder Abgabe von Stoffen zwischen dem lebenden Organismus und seiner Umgebung, der intermediäre Stoffwechsel umfasst die chemischen Umsetzungen, die innerhalb des Organismus ablaufen (Kraut \& Keller 1961). Durch die Nahrungszufuhr wird der Energiebedarf gedeckt, um in Form von energiespendenden Substanzen dem Aufbau verschiedener Gewebe sowie dem Aufbau von Enzymen und dem Ersetzen wichtiger Stoffe zu dienen (Åstrand 1987).

Eine hormonelle Steuerung der Energieaufnahme findet über das appetitanregende Hormon Ghrelin und über das Proteohormon Leptin, welches das Auftreten von Hungergefühl hemmt und den Fettstoffwechsel reguliert, statt (Shephard \& Aoyagi 2009). Des Weiteren koordinieren Hunger- und Sattheitszentren im Hypothalamus die Nahrungsaufnahme, indem sie ein Hunger- bzw. Sättigungsgefühl auslösen (Schusdziarra 2003; Golenhofen 2006; Friedrich 2007). Diese biologischen Faktoren (Füllungszustand des Magens, hormonelle Regulation, Hunger und Sättigung) zur Beeinflussung des Ernährungsverhaltens werden vervollständigt durch psychologische (emotionale und kognitive) und soziokulturelle Faktoren, womit die Anpassung an soziale und kulturelle Normen und soziodemografische Variablen zu verstehen 
sind (Pietrowsky 2006). Ernährungsgewohnheiten sind somit als stabil und schwierig veränderbar zu bezeichnen (Pudel \& Westenhöfer 2003; Schwarzer 2004).

Der Gesamtenergiebedarf eines Organismus definiert sich als Summe aus Grundumsatz und Leistungsumsatz (Kraut \& Keller 1961; Rost 2001; Krink \& Kolschewski 2011). Als Grundumsatz wird der minimale Energieumsatz des Körpers im Liegen bezeichnet, der bei neutraler Umgebungstemperatur $\left(2{ }^{\circ} \mathrm{C}\right)$ und nach zwölfstündigem Fasten gemessen wird (Rost 2001). Der Vollständigkeit halber sei der Energiebedarf für die spezifisch-dynamische Wirkung der Makronährstoffe und für den Verdauungsverlust (Konopka 2009) genannt, der allerdings aufgrund seines geringen Anteils in der gängigen Literatur nicht speziell erwähnt wird. Definiert ist der minimale Energiebedarf mit 1 kcal/ kg Körpergewicht pro Stunde (Gibala 2005). Das Maß der Energie ist kcal bzw. kJ und bezeichnet die Energie, die benötigt wird, um 1 kg Wasser um $1{ }^{\circ} \mathrm{C}$ zu erhöhen (Atzler 1927; Stone 1994b), wobei $1 \mathrm{kcal}$ in etwa 4,184 kJ entspricht (Montoye et al. 1996; Kasper 2000; Schauder 2006; Konopka 2009; Krink \& Kolschewski 2011; Graf et al. 2012).

Als Energiequellen dienen hauptsächlich Kohlenhydrate und Fette, Proteine und Alkohol zu geringerem Anteil. Eine optimale Nährstoffrelation besteht zu $60 \%$ aus Kohlenhydraten, zu $30 \%$ aus Fetten und zu $10 \%$ aus Proteinen (Kraut \& Keller 1961; Kasper 2000; DGE 2001; Rost 2001; Friedrich 2007; Konopka 2009; Krink \& Kolschewski 2011; Graf et al. 2012). Bezogen auf die Nährstoffe der einzelnen Nahrungsbestandteile gibt der physiologische Brennwert die Energiemenge an, die bei der Verbrennung von $1 \mathrm{~g}$ des betreffenden Nährstoffs im Organismus freigesetzt wird. Diese Brenn- oder Energiewerte betragen für Kohlenhydrate 4,1 kcal/ g, für Fette 9,3 kcal/ g, für Proteine 4,1 kcal/ g und für Alkohol 7,1 kcal/ g (Atzler 1927; Kraut \& Keller 1961; Valentin et al. 1979). Eine ausreichende Menge an Vitaminen, Elektrolyten und Flüssigkeit ist ebenso entscheidend für eine gesunde Ernährung, diese Nahrungsbestandteile dienen jedoch nicht als Energieträger (Rost 2001).

Wird der Energiebedarf von Faktoren wie Alter, Geschlecht, Körpergröße und körperliche Aktivität beeinflusst (Manore 2004; König \& Berg 2010; Krink \& Kolschewski 2011), entwickelt sich ein individuelles Essverhalten, das durch äußere Einflüsse geprägt wird und nicht mehr auf die Grundbedürfnisse des Organismus abgestimmt ist. So verwundert es nicht, dass in der Literatur unterschiedliche Angaben zur saisonalen Energieaufnahme gemacht werden. Ma (2006) stellt fest, dass im Herbst verglichen mit dem Frühling eine höhere Energieaufnahme von $86 \mathrm{kcal} / \mathrm{d}$ stattfindet. Andere Studien erheben im Winter eine niedrigere Energieaufnahme als im Sommer (Westerterp, Plasqui \& Goris 2005). Bei der Zusammensetzung der Nahrung konnte Ma (2006) feststellen, dass im Frühjahr mehr Kohlenhydrate und im Herbst 
mehr Fette konsumiert werden. Ein Unterschied zwischen dem Ernährungsverhalten an Werktagen und am Wochenende konnte wiederholt nachgewiesen werden (Hartman et al. 1990; Winkler et al. 1991; Haines et al. 2003; Cai et al. 2004; Schneider \& Heseker 2006). Dabei liegt die Energieaufnahme am Wochenende über der Energieaufnahme an Werktagen.

Während Cai et al. (2004) bei chinesischen Frauen kulturelle Bräuche und saisonales Angebot von Gemüse als Haupteinflüsse belegen konnten, so beeinflusst in den westlichen Industrieländern zunehmend die Globalisierung das Ernährungsverhalten. Resultat der Zivilisationskost ist eine Energiezufuhr, die über dem Energiebedarf liegt und die sich durch eine hohe Energiedichte, einen hohen Gehalt an Fett, raffinierten Kohlenhydraten, tierischem Protein, Zucker und Alkohol und nur einen geringen Gehalt an Ballaststoffen auszeichnet (Kasper 2000; de Marées 2003; Schusdziarra 2003; Konopka 2009). Konopka (2009) spricht von einer Überernährung bei gleichzeitiger Fehlernährung, die durch zu viele leere Kalorien, durch den Mangel an ballaststoffreichen Nahrungsmitteln und sekundären Pflanzenstoffen entsteht. Neben dem Angebot an Nahrungsmitteln, das nach Aussehen, Beschaffenheit und Geschmack sondiert wird (Vaclavik \& Christian 2008), orientiert sich die Entwicklung von Ernährungsmustern stark an Bezugspersonen (Benton 2004).

Die genannten Faktoren beeinflussen neben den physiologischen Faktoren die Nahrungsaufnahme, so dass eine ausgewogene Ernährung bewusst gesteuert werden muss, um sowohl quantitativ als auch qualitativ den individuellen Energiebedarf zu decken.

„Das ausschlaggebende Kriterium für eine gesundheitsgerechte, ausgewogene Ernährung liegt in der adäquaten Bedarfsdeckung an Energie und Nährstoffen. [...] Erst die Kombination und Dosierung von verschiedenen Lebensmitteln kann zu einer bedarfsgerechten Ernährung führen.“ (Pudel 1998).

Dabei werden durch eine bedarfsorientierte und aktivitätsadaptierte Auswahl der Lebensmittel Defizite in der Energieversorgung vermindert, die Belastbarkeit verbessert, leistungsmindernde Mangelerscheinungen verhindert und ein leistungsgerechtes Körpergewicht aufrecht erhalten (König \& Berg 2010). Das richtige Verhältnis von leistungsfördernden Nahrungsmitteln wird in diesem Zusammenhang als ,,individuelle Leistungskost“" bezeichnet (Konopka 2009). Da für die Frage nach der Energiebilanz nur die Nährstoffe, die als Energieträger dienen, von entscheidender Relevanz sind, wird im Folgenden auf die Makronährstoffe Kohlenhydrate, Fette und Proteine eingegangen. Ein weiterer Fokus wird auf den Flüssigkeitshaushalt gelegt, da im Allgemeinen und im Besonderen bei einer beruflichen Tätigkeit, die Witterungseinflüssen unterliegt und eine hohe körperliche Aktivität impliziert, eine ausreichende Flüssigkeitszufuhr von Bedeutung ist (McArdle et al. 2000; Hollmann \& Hettinger 2000; Manore 2004; Oberriter 2006; Graf et al. 2012). 


\subsection{Makronährstoffe}

Der Begriff Nährstoffe bezeichnet alle Substanzen, die zum Aufbau und Erhalt von Körpersubstanz, zur Energielieferung oder zum Ablauf intermediärer Stoffwechselvorgänge beitragen (Kraut \& Keller 1961) und somit dem „Betrieb der energieabhängigen zell- und gewebespezifischen Aufgaben dienen“ (Biesalski 2007). Den Nährstoffen können drei Hauptaufgaben zugeschrieben werden.

Proteine, aber auch Wasser und Eisen dienen dem Zell- und Gewebeaufbau. Für den Aufbau von Enzymen, die direkte Stoffwechselregulation und die Aufrechterhaltung der Leistungsfähigkeit sind Vitamine, Mineralstoffe und Spurenelemente zuständig. Kohlenhydrate und Fette sind die Hauptenergielieferanten und Wasser fungiert als Transportmittel und Temperaturregulator (Hollmann \& Hettinger 2000; Konopka 2009).

Neben der Unterteilung der Nährstoffe in essenzielle (Nährstoffe, die nicht vom Körper selbst gebildet werden können) und nicht essenzielle Nährstoffe, lassen sich die Nährstoffe in Makro- und Mikronährstoffe einteilen. Zu den Makronährstoffen gehören Kohlenhydrate, Proteine und Fette sowie Wasser, das jedoch in der Literatur und somit auch in der vorliegenden Arbeit gesondert betrachtet wird, da es keinen Nährwert für den Organismus besitzt (Kraut \& Keller 1961; Valentin et al. 1979; McArdle et al. 2000; de Marées 2003; Biesalski 2007; Konopka 2009; König \& Berg 2010).

In den folgenden Kapiteln werden die Funktionen von Kohlenhydraten, Fetten und Proteinen dargelegt. Neben den Referenzwerten ${ }^{1}$ der Nährstoffe wird die Relation der Nährstoffe bei der Nahrungsaufnahme unter erhöhter körperlicher Aktivität erörtert, um eine Diskussionsgrundlage für eine „,bedarfsorientierte und aktivitätsadaptierte“ (König \& Berg 2010) Ernährung zu bilden.

\subsubsection{Kohlenhydrate}

Kohlenhydrate sind die Hauptnahrungs- und Energiequelle für den menschlichen Organismus. Ihre Funktionen beinhalten Energielieferung und -speicherung (in Form von Glykogen), Freisetzung von Proteinen, Anregung des Stoffwechsels und Gewebeaufbau (z. B. Zellmembranen und Bindegewebe) (McArdle et al. 2000; Biesalski 2007; Vaclavik \& Christian 2008; Krink \& Kolschewski 2011). Kohlenhydrate werden in einfache (Monosaccharide) und komplexe (Di-, Oligo- und Polysaccharide) unterteilt, wobei Mono- und Disaccharide eine gerin-

\footnotetext{
${ }^{1}$ Der Referenzwert eines Nährstoffes gibt die Menge an, die als ausreichend erachtet wird, "bei nahezu allen gesunden Personen der Bevölkerung die lebenswichtigen metabolischen, physischen und psychischen Funktionen sicherzustellen". Hierbei handelt es sich immer um Empfehlungen, Schätzwerte und Richtwerte, die einem Defizit vorbeugen sollen (Biesalski 2007).
} 
gere Nährstoffdichte aufweisen und deshalb einen geringeren Anteil bei der Nahrungsaufnahme ausmachen sollten, um eine qualitativ optimale Zusammensetzung der Kohlenhydrate zu gewährleisten (Stone 1994a; de Marées 2003; Oberriter 2006; Vaclavik \& Christian 2008; Graf et al. 2012). Komplexe Kohlenhydrate befinden sich z. B. in Gemüse, Vollkornprodukten, ungeschältem Reis. Der Vorteil von einfachen Kohlenhydraten (z. B. Brötchen mit Marmelade) liegt jedoch darin, dass der Organismus sofort Energie gewinnen kann. Ein hieraus resultierender Nachteil ist ein Blutzuckerabfall ca. zwei Stunden nach Nahrungsaufnahme (Rost 2001).

Der Kohlenhydratanteil in der Ernährung sollte ca. $50 \%$ betragen, bei erhöhter körperlicher Tätigkeit bis zu $60 \%$, da bei hoher körperlicher Aktivität der Energiegewinn aus den Kohlenhydraten ansteigt (Åstrand 1987; Stone 1994a; Kasper 2000; McArdle et al. 2000; Manore 2004; Friedrich 2007; Konopka 2009). Laut Konopka (2009) liegt der durchschnittliche prozentuale Anteil der Kohlenhydrate der Grundnährstoffe in der Zivilisationskost mit ca. $35 \%$ deutlich unter dem empfohlenen Wert. Auch bei griechischen Forstwirten wird von Gallis \& Panagopoulou (2007) eine zu geringe Kohlenhydratzufuhr festgestellt. Referenzwerte für Kohlenhydrate differieren in der Literatur. Eine Menge von 4 g/ kg Körpergewicht (mit dem Verweis auf die Relevanz der Beachtung der qualitativen Diskrepanz zwischen einfachen und komplexen Kohlenhydraten) (Schauder 2006) bildet den niedrigsten Wert, gefolgt von 5-6 g/ kg Körpergewicht bei aktiven Menschen (Gibala 2005) und 5-7 g/ kg Körpergewicht bei moderater Aktivität bis hin zu 7-12 g/ kg Körpergewicht bei hoher Aktivität (Manore 2004). Sollte es zu einer zu hohen Kohlenhydrataufnahme kommen, so werden überflüssige Kohlenhydrate nur in begrenztem Maß als Glykogen und überwiegend in umgewandelter Form als Fett eingelagert (̊̊strand 1987; Stone 1994a).

Eine Wiederauffüllung des Glykogendepots findet über die Nahrungsaufnahme statt und ist erstens abhängig vom Zeitpunkt der Kohlenhydrataufnahme. Eine Aufnahme vor der körperlichen Beanspruchung erweist sich dabei als optimal (Drew \& Cleary 2005). Einen zweiten Aspekt stellt die Menge der zugeführten Kohlenhydrate dar (s. o.). Der dritte Aspekt verweist auf die Art der Kohlenhydrate und ihren glykämischen Index ${ }^{2}$, wobei ein hoher glykämischer Index für die Wiederauffüllung des Glykogendepots besonders während der Regeneration von Vorteil ist (Hollmann \& Hettinger 2000; Burke et al. 2004).

\footnotetext{
${ }^{2}$ Der Glykämische Index ist ein Maß zur Bestimmung der Potenz eines Kohlenhydrates, eine bestimmte Menge an Blutzucker frei zu setzen. Der vermehrte Konsum von Kohlenhydraten mit hohem glykämischen Index (z. B. Zucker, Weißmehl, Mais) führt zu einer gestörten Glukosetoleranz (Zalpour 2002).
} 


\subsubsection{Fette}

Die Bedeutung der Fette liegt in ihrer Eigenschaft als größter und wichtigster Energiespeicher des Organismus. Des Weiteren gibt Fett den Zellmembranen die Struktur, ist Lieferant essenzieller Fettsäuren, dient dem Transport fettlöslicher Vitamine, synthetisiert andere biologisch wirksame Substanzen (z. B. Cholesterin und Steroidhormone), dient als Geschmacks- und Texturstoff, als Wärmeschutz und als mechanischer Schutz für empfindliche Organe (Stone 1994a; Kasper 2000; McArdle 2000; Rost 2001; Biesalski 2007; Vaclavik \& Christian 2008; Konopka 2009; Krink \& Kolschewski 2011). Die Energiegewinnung findet über die Oxidation von Fettsäuren besonders in Ruhe und bei langandauernder körperlicher Arbeit statt (Hollmann \& Hettinger 2000).

Fette werden in ungesättigte (v. a. pflanzliche Fette), gesättigte (in tierischen Fetten vermehrt vorhanden) und in Transfettsäuren (synthetische Herstellung) eingeteilt. Bei der Ernährung sollte auf die Relation der Fettsäuren geachtet werden, da der Verzehr von gesättigten Fettsäuren zu einer Erhöhung des Cholesterinspiegels und der Verzehr von Transfettsäuren zu einer Erhöhung des LDL-Cholesterin-Spiegels im Blut führen kann und somit langfristig das Risiko für koronare Herzerkrankungen steigt (Rost 2001; Koh-Banerjee et al. 2003; Gibala 2005).

Ähnlich wie bei den Kohlenhydraten kommt es bei den Fetten auf die richtige Dosierung an. Der prozentuale Anteil von Fett an den Grundnährstoffen sollte bei ca. 30 \% liegen. Angaben in der Literatur bewegen sich zwischen 20 und $35 \%$ der Energieaufnahme (Stone 1994a; Kaspar 2000; Manore 2004; Schauder 2006; Biesalski 2007; Friedrich 2007; Konopka 2009). Der Ist-Zustand des prozentualen Anteils beträgt in der Zivilisationskost 40-45 \% (Konopka 2009) und liegt somit deutlich über dem Referenzwert. Überschüssiges Fett wird im Unterhautfettgewebe gespeichert und kann nach der Aufspaltung als Fettsäure und Glycerol gespeichert werden (Åstrand 1987). Das aufgenommene Fett sollte sich zu zwei Dritteln aus ungesättigten und zu einem Drittel aus gesättigten Fettsäuren zusammensetzen (Stone 1994a; de Marées 2003), wobei bezogen auf das Körpergewicht eine Menge von 1 g/ kg als Referenzwert dient (Schauder 2006).

Jedoch spielt bei den Fetten die qualitative Zusammensetzung im Vergleich zu den Kohlenhydraten eine unwichtigere Rolle. Relevant ist die Quantität, da eine uneingeschränkte Menge mit einem hohen Energiegehalt vorhanden ist, die Freisetzung der Energie allerdings sehr langsam stattfindet und abhängig von den Kohlenhydraten ist (Graf et al. 2012).

Ein Anstieg der Fettoxidation kann durch eine verminderte Nahrungszufuhr erreicht werden, mit der die Kohlenhydratoxidation abnimmt und die Energie aus den Energiespeichern in Form von Fett gewonnen wird (Hall 2010). Des Weiteren wird eine eher geringere Fettzufuhr 
bei erhöhter körperlicher Aktivität empfohlen (de Marées 2003; Konopka 2009), um den notwendigen höheren prozentualen Anteil der Kohlenhydrate zu ermöglichen. Bei Schwerstarbeit jedoch bedarf es einer fettreichen Nahrungszufuhr, um den sehr hohen Energieverbrauch durch energiereiche Nahrung decken zu können (Golenhofen 2006).

\subsubsection{Proteine}

Ebenfalls zur Energiegewinnung im menschlichen Organismus tragen die Proteine, bestehend aus Aminosäuren (Stone 1994a; Vaclavik \& Christian 2008), bei. Im Gegensatz zu den Kohlenhydraten ist die Energiegewinnung aus Proteinen mit einem hohen Energieaufwand verbunden und wird daher nur in Ausnahmefällen zur Energiegewinnung herangezogen (Stone 1994a; Rost 2001; Krink \& Kolschewski 2011; Graf et al. 2012). Hauptsächlich dienen Proteine als Funktionsstoffe. Zum einen fungieren sie als Strukturbestandteile in Zellen und Geweben und sind wichtig für den Ersatz und den Neuaufbau eiweißhaltiger Substanzen wie z. B. der kontraktilen Elemente der Muskulatur (Stone 1994a; Kasper 2000; McArdle et al. 2000; Rost 2001; Oberriter 2006; Konopka 2009; Krink \& Kolschewski 2011; Graf et al. 2012; Koch 2012). Zum anderen dienen Proteine als Stoffwechselregulatoren in Form von Hormonen und Enzymen und steuern vermehrt bei körperlicher Aktivität verschiedenste Stoffwechselvorgänge (Kasper 2000; McArdle et al. 2000; Rost 2001; Oberriter 2006; Vaclavik \& Christian 2008; Konopka 2009; Krink \& Kolschewski 2011; Graf et al. 2012). Mit dem Aufbau von Antikörpern kommt den Proteinen auch bei der Immunfunktion eine große Bedeutung zu (McArdle et al. 2000; Rost 2001; Konopka 2009; Krink \& Kolschewski 2011; Graf et al. 2012). Darüber hinaus sind Proteine Trägerstoffe und transportieren verschiedene Substanzen wie z. B. Triglyzeride, fettlösliche Vitamine und Eisen (McArdle et al. 2000; Rost 2001; Krink \& Kolschewski 2011), haben eine Pufferfunktion im Säure-Basen-Haushalt inne und regulieren den Flüssigkeitshaushalt u. a. über den kolloidosmotischen Druck (Rost 2001; Oberriter 2006).

Hinsichtlich der Eiweißaufnahme gibt es ein Bilanzminimum, das die minimale Eiweißmenge angibt, die der Mensch zu sich nehmen muss, um eine ausgeglichene Bilanz zwischen Eiweißaufnahme und -abbau zu erreichen. Dieses Bilanzminimum beträgt 0,5 g/ kg Körpergewicht pro Tag (Golenhofen 2006). Die Angaben für eine optimale Eiweißzufuhr bei einem durchschnittlichen Bedarf liegen bei 0,8 g/ kg Körpergewicht pro Tag (Hollmann \& Hettinger 2000; Kasper 2000; McArdle et al. 2000; Rost 2001; de Marées 2003; Manore 2004; Gibala 2005; Schauder 2006; Biesalski 2007; Friedrich 2007;Konopka 2009; Koch 2012), wobei der prozentuale Anteil an der Gesamtenergiemenge 10-15\% betragen sollte (Stone 1994a; McArdle et al. 2000; Manore 2004; Friedrich 2007; Konopka 2009). 
Die optimale Zufuhr von Proteinen steigt mit der Erhöhung der körperlichen Aktivität aufgrund des Verlusts an strukturellen und enzymatischen Proteinen (Stone 1994a) und der Vergrößerung der aktiven Zellmasse (Kraut \& Keller 1961). So kann bei griechischen Forstwirten eine erhöhte Proteinzufuhr festgestellt werden (Gallis \& Panagopoulou 2007). Eine zu hohe Eiweißzufuhr (nicht mehr als 3 g/ kg Körpergewicht pro Tag) sollte jedoch vermieden werden, da sie gesundheitsschädigend auf den Organismus wirkt und die Aufspaltungsprodukte überschüssiger Proteine zum Aufbau von Glykogen und Fetten verwendet werden und auch in dieser Form gelagert werden, so dass sich die Körperfettmasse erhöht (Åstrand 1987; Stone 1994a; Graf et al. 2012). Um einem Abbau von Muskelgewebe vorzubeugen, sollte auf eine ausreichende Zufuhr von biologisch hochwertigem Eiweiß und Kombinationen aus pflanzlichen und tierischen Eiweißen geachtet werden (Wirth 2003).

Der Proteinbedarf erhöht sich bei körperlicher Belastung, um zerstörte Muskelfasern wieder aufbauen zu können, für einen Muskelzuwachs zu sorgen und um die erforderliche Energie bereit zu stellen (Lemon 1998; Manore 2004). Aufgrund der insgesamt ansteigenden Ernährungszufuhr bei körperlicher Belastung bleibt der prozentuale Anteil der Proteine mit 10-15 $\%$ des Gesamtkalorienumsatzes konstant (Hollmann \& Hettinger 2000). Für Menschen mit mehreren Stunden (Trainings-) Belastung am Tag entspricht dies einem Wert von 1,41,7 g/ kg Körpergewicht (Hollmann \& Hettinger 2000). Bei der Belastungsart wird unterschieden in Ausdauerbelastungen mit einer durchschnittlichen empfohlenen Proteinzufuhr von bis zu 1,5 g/ kg Körpergewicht (Lemon 1991; Stone 1994a; McArdle et al. 2000; Rost 2001; de Marées 2003; Manore 2004; Gibala 2005; Konopka 2009; Graf et al. 2012) und Kraftsport. Die Empfehlung für Kraftsportler liegt zwischen 1,5-2,0 g/ kg Körpergewicht (Lemon 1991; Stone 1994a; de Marées 2003; Gibala 2005; Konopka 2009), wobei Gewichthebern zu früherem Zeitpunkt Werte von bis zu 4,0 g/ kg Körpergewicht empfohlen wurden (Stone 1994a; Spendlove et al. 2015). Neuere Studien haben jedoch ergeben, dass selbst bei schwerer körperlicher Belastung die Proteinzufuhr einen Wert von 2,0 g/ kg Körpergewicht nicht überschreiten sollte, um gesundheitsschädigende Konsequenzen wie z. B. Arteriosklerose und Nierensteine zu vermeiden und weil es keine wissenschaftliche Begründung gibt, dass eine noch höhere Proteinzufuhr den Muskelzuwachs oder die Kraftleistungsfähigkeit fördert (Rost 2001; Berg \& König 2006; Graf et al. 2012). Hieran knüpft die Forderung nach wissenschaftlich fundierten Untersuchungen zu dieser Thematik (Spendlove et al. 2015).

Die biologische Wertigkeit von Proteinen gibt an, wie viel Gramm Körperstickstoff durch 100 g resorbierten Nahrungsstickstoff ersetzt oder gebildet werden können bzw. wie viel Gramm Körpereiweiß durch $100 \mathrm{~g}$ des betreffenden Nahrungseiweißes aufgebaut werden 
können (Kasper 2000; Konopka 2009). Dementsprechend ist die biologische Wertigkeit hoch, wenn alle essenziellen Aminosäuren ausreichend in Proteinen vorhanden sind, die für die Synthese von eiweißhaltigen Substanzen notwendig sind (Stone 1994a; Golenhofen 2006; Biesalski 2007). Folglich ist neben der Menge der Proteinzufuhr auch die Qualität der zugeführten Proteine relevant. Tierische Proteine sind hochwertiger als pflanzliche, jedoch steigt die Wertigkeit bei einer Mischung von Eiweißstoffen verschiedener Nahrungsstoffe, so dass eine Kombination aus tierischen und pflanzlichen Proteinen zu jeweils $50 \%$ empfohlen wird (Kraut \& Keller 1961; Stone 1994a; de Marées 2003; Burke et al. 2004; Tipton \& Wolfe 2004; Schauder 2006; Konopka 2009). Darüber hinaus scheint der Zeitpunkt der Proteinaufnahme gekoppelt mit der Aufnahme von Kohlenhydraten eine Auswirkung auf die optimale Verwendung der Proteine im Organismus zu haben (Burke et al. 2004; Tipton \& Wolfe 2004). Der Zeitpunkt der Mahlzeiten sollte an die körperliche Anforderung angepasst werden, die Proteinzufuhr möglichst nach körperlicher Beanspruchung stattfinden (Biolo et al. 1995; Drew \& Cleary 2005; Etheridge et al. 2008). Studien belegen, dass eine höhere Eiweißzufuhr mit einer erhöhten Muskelkraft korreliert (Thompson et al. 2015). Eine Proteinzufuhr direkt nach einer Belastung führt zu einer Kraftsteigerung und zu einer schnelleren Erholung der Muskulatur (Biolo et al. 1995; Etheridge et al. 2008; Thompson et al. 2015). Darüber hinaus besitzen Proteine mit $30 \%$ eine hohe spezifisch-kalorische Wirkung, so dass ein hoher energetischer Anteil der Nahrungsstoffe für die Wärmebildung genutzt werden kann. Aus diesem Grund ist eine eiweißreiche Ernährung besonders bei Kälte günstig (Golenhofen 2006).

Proteine sind somit Funktionsstoffe, die sowohl für die Energiebereitstellung als auch für den Aufbau eiweißhaltiger Substanzen zuständig sind (Graf et al. 2012). Die Regulation der Zellmasse im menschlichen Organismus wird durch die Proteinsynthese und den Proteinabbau gesteuert, so dass der Proteinbestand konstant ist, sich jedoch im ständigen Umsatz befindet (Koch 2012). Um ein Gleichgewicht in diesem System zu gewährleisten, ist eine ausreichende Deckung des Kalorien- und des Proteinbedarfs (besonders bei körperlicher Mehrbeanspruchung) notwendig (Kraut \& Keller 1961).

\subsubsection{Flüssigkeit}

Der Wasserhaushalt ist neben den Spurenelementen wichtig für die Leistungsfähigkeit des Menschen und die Steuerung und Aufrechterhaltung des Stoffwechsels (Åstrand 1987; Manore 2004; Konopka 2009). Als konkrete Funktionen sind die Transportfunktion, die Diffusion von Gasen über Wasser, das Ausscheiden von Stoffwechselendprodukten, die Temperaturregulation und die Formgebung zu nennen (McArdle et al. 2000). 
Der Flüssigkeitsbedarf eines Menschen hängt von der Körpermasse, der körperlichen Aktivität und der Umgebungstemperatur ab. Bei normaler körperlicher Aktivität und mittlerer Temperatur geht man von einem Bedarf von $1 \mathrm{ml} /$ Kalorie Energieaufnahme aus, wobei ca. 1 Liter Flüssigkeit über Lebensmittel und Stoffwechselwasser aufgenommen wird und die restliche Menge über das Trinken dem Körper zugeführt wird (Rost 2001; Graf et al. 2012). Ein Zusammenhang zwischen körperlicher Aktivität und Wasserverlust konnte bei Männern in einer Studie belegt werden (Westerterp et al. 2005). Die Flüssigkeitszufuhr sollte über Leitungswasser, Mineralwasser, Früchte- und Kräutertees und verdünnte Obst- und Gemüsesäfte stattfinden (Grunewald 2006). Bei körperlicher Arbeit ist sowohl die Quantität der Flüssigkeitszufuhr (Hollmann \& Hettinger 2000; McArdle et al. 2000; Manore 2004; Oberriter 2006; Graf et al. 2012) zu erhöhen als auch die Art der Getränke anzupassen. Es sollte darauf geachtet werden, dass neben den o. g. Getränken (Wasser und mineralstoffreiche Getränke wie z. B. Cola, Limonade, Tee) auch flüssigkeitsreiches Obst aufgenommen wird, um den Bedarf an Mineralstoffen und Spurenelementen zu decken (Drew \& Cleary 2005; Konopka 2009). Während des Trainings bzw. der Belastung empfiehlt Gibala (2005) eine Flüssigkeitszufuhr von 150-350 ml in einem Abstand von 15-20 Minuten. Bates et al. (2013) konnten bei Holzfällern eine Dehydrierung während der Arbeitszeit und einen Konsum nicht geeigneter Flüssigkeiten zum Ausgleich des Wasserhaushaltes feststellen.

In den Bereich der Flüssigkeitszufuhr fällt auch die Menge an konsumierten alkoholischen Getränken. Der Konsum von Alkohol birgt grundsätzlich ein Risiko für die Entstehung alkoholbedingter gesundheitlicher Störungen. Es liegen jedoch Angaben zu Grenzwerten für den Alkoholkonsum vor, bei denen dieses Risiko relativ niedrig ist. Diese Definition riskanter Alkoholmengen liegt für Männer bei 24 g/ d (BZGA 2011). Erhöhter Alkoholkonsum kann neben psychischen, körperlichen und sozialen Störungen (ebd.) zu einer Minderung der Leistungsfähigkeit führen (WHO 2014), weshalb eine Betrachtung der konsumierten Alkoholmenge in dieser Untersuchung relevant ist.

Es bleibt festzuhalten, dass eine konstante Flüssigkeitsbilanz ausschlaggebend für die Leistungsfähigkeit des menschlichen Organismus ist. Bei einer negativen Bilanz sinken die maximale erreichbare Belastungsstufe und die mögliche Arbeitsausdauer (Hollmann \& Hettinger 2000), so dass zum einen Studien zum Thema Dehydrierung bei Berufen mit hoher körperlicher Beanspruchung (wie z. B. Forstwirte) wünschenswert wären (Wästerlund 1998) und zum anderen eine ständige Balance zwischen Flüssigkeitsabgabe und -zufuhr besonders bei körperlicher Aktivität von Bedeutung ist. 


\subsection{Zusammenfassung}

Zivilisationskost ist laut Konopka (2009) mit einer zu geringen Kohlenhydrataufnahme und einer zu hohen Fettaufnahme ein Beispiel für eine schlechte Ernährungsweise. Die Empfehlung für die Nährstoffrelation der Gesamtenergiemenge wird in der Literatur mit 55-60\% aus Kohlenhydraten, 25-30 \% aus Fetten und 10-15\% aus Proteinen angegeben (Hollmann \& Hettinger 2000; de Marées 2003; Gibala 2005; Golenhofen 2006; Oberriter 2006). Für eine ausgewogene Ernährung ist neben der Quantität auch die Qualität der Nährstoffe relevant. Es sollten bevorzugt Kohlenhydrate als Stärke mit einem hohen Anteil an Ballaststoffen aufgenommen werden; bei der Proteinzufuhr ist ein gutes Mischungsverhältnis zwischen den einzelnen Aminosäuren und bei den Fetten der Gehalt an gesättigten Fettsäuren, der maximal ein Drittel der Fettsäuren ausmachen sollte, ausschlaggebend für eine optimale Ernährung (Golenhofen 2006).

Bei einer aktiven Lebensweise sollte die Kohlenhydratzufuhr auf Kosten der Protein- und Fettzufuhr leicht erhöht werden (Berg \& König 2006; Konopka 2009). Der Richtwert für die Energiezufuhr liegt bei $2400 \mathrm{kcal} / \mathrm{d}$ und nimmt im Alter leicht ab. Bei Mittelschwerarbeitern (zusätzlich 600 kcal), Schwerarbeitern (zusätzlich 1200 kcal) und Schwerstarbeitern (zusätzlich $1600 \mathrm{kcal}$ ) kommt es zu einem Anstieg des Energiebedarfs, der im Bereich der Schwerarbeit mit dem Energiebedarf eines Sportlers verglichen werden kann (Hollmann \& Hettinger 2000; Kasper 2000).

Unter einer ausgewogenen Ernährung versteht man eine bedarfsangepasste und vollwertige Nahrungszufuhr. Wichtige Aspekte hierbei sind die Gewährleistung einer ausgeglichenen Energiebilanz (vgl. Kapitel 4), Ausschluss von Mangelsituationen und die Zufuhr aller lebensnotwendigen Nährstoffe, angepasst an den individuellen Bedarf (de Marées 2003; Vaclavik \& Christian 2008; Krink \& Kolschewski 2011).

Grundsätzlich steigert eine optimale Ernährung die Gesundheit und verbessert die Leistungsfähigkeit (Sluik et al. 2008; Konopka 2009; Çalişkan \& Çağlar 2010). Bei körperlicher Belastung sollte beachtet werden, dass die Ernährung fettarm und reich an biologisch hochwertigem Eiweiß ist. Des Weiteren sollte unter Betrachtung der regenerativen Wirkung von Ernährung auf den Zeitpunkt der Nahrungsaufnahme geachtet werden (Konopka 2009).

Unter den Voraussetzungen einer an die körperliche Aktivität angepassten optimalen Ernährung befasst sich das folgende Kapitel mit dem Energieverbrauch und seinen bestimmenden Faktoren. 


\section{Energieumsatz}

Für die Erhaltung der Körpertemperatur, die unwillkürlichen Muskelkontraktionen (z. B. Atmung), die Stoffwechsel- und Verdauungsvorgänge und für die Muskelaktivität verbraucht der menschliche Organismus Energie (Montoye et al. 1996).

Physikalisch betrachtet ist der Energieumsatz eine Leistung (in W), die als Arbeit pro Zeit definiert ist (Heck 1990; Hollmann \& Hettinger 2000; de Marées 2003; Golenhofen 2006; Persson 2007), die in Abhängigkeit zur Körpergröße, Bewegungsintensität, Körperkomposition, Mobilisation der Substrate, Änderung der Blutfette und zur kardiovaskulären Funktion steht (Stone 1994b). Die Energie ist dabei die Fähigkeit, Arbeit zu verrichten, wobei die Arbeit das Produkt aus Kraft und Weg ist und in Joule angegeben wird (Heck 1990; Persson 2007; Kroidl et a. 2010).

Betrachtet man die Leistung aus Sicht des biopsychosozialen Modells, so werden bei dieser Perspektive „die komplex miteinander verknüpften physisch-energetischen, motorischen und technisch-koordinativen Faktoren ebenso wie affektiv, psychosozial und äußerlich (sozial, kulturell, klimatisch) determinierten Einflüsse“ (Koch 2012) berücksichtigt. Die Leistungsfähigkeit eines Menschen hängt somit vom Leistungsvermögen und von der Leistungsbereitschaft ab und wird von der Art und Umsetzung der Aktivität determiniert (Hollmann \& Hettinger 2000; Golenhofen 2006; Koch 2012).

Im Bereich der Forstwirtschaft wird die Normalleistung eines Forstwirtes als die Leistung definiert,

\footnotetext{
"die von jedem geeigneten, geübten und voll eingearbeiteten Waldarbeiter mit ordnungsgemäßen Werkzeug und im zweckmäßigen Arbeitsablauf unter Wahrung der Betriebssicherheit ohne Gesundheitsschädigung auf Dauer des Arbeitslebens im Durchschnitt der täglichen Arbeitszeit erreicht und erwartet werden kann, wenn die Arbeitszeit und die den Vorgabezeiten enthaltenen Verteil- und Erholungszeiten eingehalten werden" (REFA 2004, zitiert in Sohns 2012).
}

Der Gesamtumsatz eines Menschen bildet sich aus der Summe von Grundumsatz und Arbeitsumsatz (Lehmann 1961c, Persson 2007). Der Grundumsatz ist die Energie, die zur Aufrechterhaltung der physiologischen Homöostase benötigt wird (Erhalt der Körpertemperatur, unwillkürliche Muskelkontraktionen) (Kreymann 2006) und beträgt ca. 1 W/ kg Körpergewicht pro Tag (Golenhofen 2006; Friedrich 2007; Persson 2007) bzw. einer Sauerstoffaufnahme ( $\left.\mathrm{VO}_{2}\right)$ von 3,5 ml/ kg Körpergewicht/ min (Montoye et al. 1996; Platen 2001; Arena et al. 2007; Kroidl et al. 2010). Er wird unter folgenden standardisierten Bedingungen gemessen: morgens, bei einer Nahrungskarenzzeit von zwölf Stunden, liegend, bei psychischer Indifferenz, bei indifferenter Raumtemperatur (Lehmann 1961c; Kasper 2000; McArdle et al. 
2000; Golenhofen 2006; Kreymann 2006; Konopka 2009). Der bei aktiven Menschen höhere gemessene Grundumsatz resultiert aus gesteigerten regenerativen Prozessen (Konopka 2009). Darüber hinaus wird ein erhöhter Grundumsatz nach ungewohnten Belastungen, die zur Verletzung von Muskelfasern führen, gemessen. Grund für den erhöhten Grundumsatz ist auch hier die Regeneration in Form von der Zersetzung und Resynthetisierung der zerstörten Fasern (Burt et al. 2013).

Der Ruheenergieumsatz wird hingegen unter weniger standardisierten Bedingungen gemessen (körperliche Ruhe, längere Nahrungskarenz) und beträgt ca. $10 \%$ mehr als der Grundumsatz (Montoye et al. 1996; de Marées 2003; Golenhofen 2006; Kreymann 2006). Die sich daraus ergebende Sauerstoffaufnahme beträgt $3,85 \mathrm{ml} / \mathrm{kg} \mathrm{KG/} \mathrm{min.} \mathrm{Um} \mathrm{hieraus} \mathrm{einen} \mathrm{Energieum-}$ satz in kcal berechnen zu können, wird der $\mathrm{O}_{2}$-Verbrauch pro Minute mit dem kalorischen Äquivalent $(\mathrm{K} \ddot{\mathrm{A}})^{3}$ multipliziert, wobei das Mengenverhältnis der Nahrung berücksichtigt wird. Legt man die empfohlene Nahrungszusammensetzung von $10 \%$ Eiweiß, $30 \%$ Fett und $60 \%$ Kohlenhydrate zugrunde, so ergibt sich folgendes kalorisches Äquivalent (vgl. de Marées 2003):

$$
\left(0,6 * 21 \mathrm{~kJ} / 1 \mathrm{O}_{2}\right)+\left(0,3 * 19,7 \mathrm{~kJ} / 1 \mathrm{O}_{2}\right)+\left(0,1 * 18,8 \mathrm{~kJ} / 1 \mathrm{O}_{2}\right)=20,39 \mathrm{~kJ} / 1 \mathrm{O}_{2}
$$

In kcal umgerechnet ergibt dies einen Wert von 4,877 kcal/ $1 \mathrm{O}_{2}$. Mit dem Wissen über die Zusammensetzung der Nahrung lassen sich somit aus der durchschnittlichen $\mathrm{O}_{2}$-Aufnahme von 3,85 ml/ kg KG/ min der Grund- als auch der Ruhenergieumsatz abschätzen. Bei einem $80 \mathrm{~kg}$ schweren Mann beträgt der Grundumsatz $280 \mathrm{ml} / \mathrm{min}$ bzw. 1,36 kcal/ min und der Ruheumsatz $308 \mathrm{ml} / \mathrm{min}$ bzw. 1,41 kcal/ min.

Umsatzsteigerungen können durch psychische Anspannung, allgemeine Beschleunigung der Stoffwechselprozesse und bei hohen Temperaturen durch die Steigerung der Herzleistung eintreten (Golenhofen 2006). Der größte Einfluss auf den Energieumsatz entsteht jedoch durch körperliche Aktivität, womit sich das folgende Kapitel eingehend befasst.

\subsection{Energieumsatz bei körperlicher Aktivität}

Körperliche Aktivität ist die Hauptursache für einen Anstieg des Energieumsatzes. Gekennzeichnet ist ein solcher Anstieg durch einen erhöhten Sauerstoffverbrauch infolge muskulärer Beanspruchung, der auch als „Arbeit im biologischen Sinne“ (Hollmann \& Hettinger 2000)

\footnotetext{
${ }^{3}$ Das kalorische Äquivalent bezeichnet diejenige Energiemenge in Joule, „die bei der Reaktion der betreffenden Substanz mit 1 Liter Sauerstoff freigesetzt wird“ (de Marées 2003). Um eine Vergleichbarkeit mit weiteren erhobenen Werten zu ermöglichen, wird dieser Wert in dieser Untersuchung in kcal umgewandelt. Für eine durchschnittliche Nahrungszufuhr wird ein kalorisches Äquivalent von 4,85 kcal/ $1 \mathrm{O}_{2}$ angegeben (ScharhagRosenberger \& Schommer 2013; vgl. auch Atzler 1927).
} 
bezeichnet wird. Körperliche Aktivität kann in Watt, in Zeit und in Anzahl von Bewegungen ausgedrückt werden (Montoye et al. 1996). Dabei ist der Energieumsatz bei körperlicher Belastung abhängig von Belastungsdauer, -frequenz, -intensität, -art als auch von den Umständen der Aktivität wie z. B. individuellen Voraussetzungen (Körpermasse, Bewegungsökonomie) und äußeren Einflüssen (klimatische Bedingungen, Luftwiderstand, Bodenbeschaffenheit, Geländeprofil) (Montoye et al. 1996; McArdle et al. 2000; de Marées 2003).

Es können bei schwerer körperlicher Arbeit Werte erreicht werden, die das 20fache des Grundumsatzes ausmachen (Hallhuber 1971; Golenhofen 2006; Kreymann 2006; Persson 2007). Körperliche Höchstleistungen können jedoch nicht über einen längeren Zeitraum ausgeübt werden (Lehmann 1961c; Kreymann 2006). „Die Leistungsfähigkeit des Organismus ist durch die Bereitstellung von Energie und Sauerstoff für den Muskel begrenzt“" (Hick \& Hick 2002; vgl. auch Atzler 1927; Lehmann 1961c; Mellerowicz 1979). Die Dauerleistungsgrenze trennt die Schwere der statischen als auch dynamischen Arbeit in zwei Bereiche (vgl. Abbildung 1). Der Bereich unterhalb der Dauerleistungsgrenze beschreibt die Arbeit, die ohne muskuläre Ermüdung über mindestens acht Stunden ausgeübt werden kann. Dies ist nur möglich, wenn eine Balance zwischen Verbrauch und Versorgung des Muskels mit Substraten vorliegt (ebd.). Findet die muskuläre Arbeit oberhalb der Dauerleistungsgrenze statt, fehlt diese Balance. Aufgrund der fehlenden Balance ist die Arbeit zeitlich begrenzt. Diese Grenze tritt früher ein, wenn die Intensität der Arbeit steigt (Hick \& Hick 2002; Boutellier \& Ulmer 2005). Abbildung 1 zeigt die Aufteilung des täglichen Energiebedarfs bei unterschiedlich schwerer Arbeit. Die Höhe unterschiedlicher Belastungen wird ins Verhältnis zum Grundumsatz gesetzt, so dass anhand eines Multiplikationsfaktors ${ }^{4}$ (Metabolic Equivalent of Task = MET) gegebene Belastungen berechnet werden können (Ainsworth 1993; Montoye et al. 1996).

\footnotetext{
${ }^{4}$ Der Schätzwert des Grundumsatzes von 3,5 $\mathrm{ml} \mathrm{O}_{2}$ pro kg Körpergewicht pro Minute entspricht 1 MET (Mellerowicz 1979; Platen 2001; Arena et al. 2007; Fialka-Moser 2013).
} 


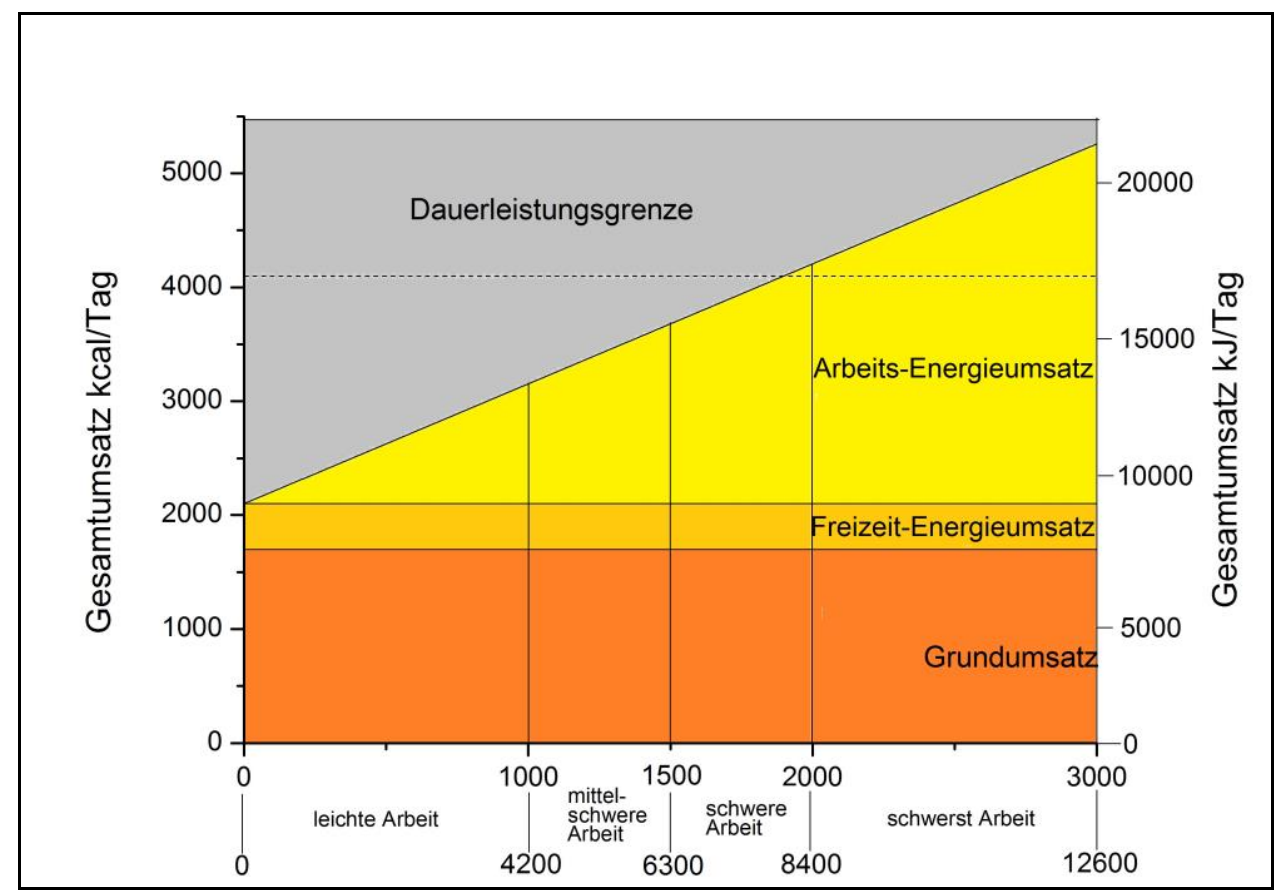

Abbildung 1: Aufteilung des täglichen Energiebedarfs bei leichter, mittelschwerer, schwerer u. schwerster Arbeit mit Angaben der Dauerleistungsgrenze. ${ }^{5}$

Für die Berechnung des Energieumsatzes bzw. um $\mathrm{VO}_{2}$-Schätzwerte mittels MET bilden zu können, benötigt man den Ruheumsatz ${ }^{6}$ einer Person (Pettitt et al. 2007). Bei der indirekten Kalorimetrie wird zwar zu allen Messzeitpunkten zu Beginn der Messung der Proband in sitzender Position gemessen. Allerdings entspricht dieser Wert weder dem Grundumsatz noch dem Ruheumsatz, da die Probanden zu Beginn ihres Arbeitstages keine Nahrungskarenz von zwölf Stunden aufweisen und z. T. bereits körperlich aktiv waren. Hinzu kommt die psychische Komponente, resultierend aus der für die Probanden ungewohnten Situation. Aus diesem Grund wird der Ruheumsatz anhand einer Formel berechnet. Formeln, die den Körperfettanteil berücksichtigen, überschätzen den Ruheumsatz und zeichnen sich hauptsächlich bei ausdauertrainierten Athleten aus (Thompson \& Manore 1996; de Lorenzo et al. 1999; McArdle et al. 2000; Kreymann 2006; Dunford \& Doyle 2007; Laquale 2007; Koshimizu et al. 2012). Für die Berechnung des Ruheumsatzes (RMR = Resting Metabolic Rate) der Forstwirte wird deshalb die Formel (für Männer):

$$
\mathrm{RMR}=10 * \mathrm{Wt}(\mathrm{kg})+6,25 \mathrm{Ht}(\mathrm{cm})-5 * \text { age }(\mathrm{yrs})+5
$$

nach Mifflin et al. (1990) verwendet, welche auch die Basis für die Formel der WHO bildet und welche sich in einer vergleichenden Untersuchung als die exakteste Formel zur Berechnung des Ruheumsatzes herausgestellt hat (Frankenfield et al. 2003). Wt steht in der Formel

\footnotetext{
5 Nach Hollmann \& Hettinger 2000; vgl. auch Lehmann 1961c.

${ }^{6}$ Der Begriff des Ruheumsatzes (RMR = Resting Metabolic Rate) meint in der Literatur oftmals den Grundumsatz. So ist die angeführte Berechnung der RMR an dieser Stelle als Wert für den Grundumsatz der Probanden zu verstehen.
} 
für das Körpergewicht in kg, Ht gibt die Körpergröße in $\mathrm{cm}$ an und age bezeichnet das Alter in Jahren. Der berechnete Ruheumsatz entspricht dabei 1 MET, so dass mit Hilfe der Tabellen nach Ainsworth $(1993,2000,2011)$ die entsprechenden Energieumsätze für die einzelnen Probanden geschätzt werden können. Mit dieser Berechnung werden individuelle Werte für den Energieumsatz erzielt, die als Grundlage für die Berechnung des täglichen Energieumsatzes der einzelnen Probanden dient.

Eine weitere Möglichkeit zur Klassifizierung des Energieumsatzes liegt in der Bestimmung der Intensität der körperlichen Aktivität nach PAL (= physical activity level) (McArdle et al. 2000; Oberriter 2006). PAL kennzeichnet den durchschnittlichen täglichen Energiebedarf für körperliche Aktivität als Mehrfaches des Grundumsatzes (Krink \& Kolschewski 2011). Die bei solch einer vorgenommenen Berechnung zugrunde gelegten Grundumsätze werden entweder gemessen oder anhand einer Formel geschätzt (Montoye et al. 1996).

Bei leichter bis mittelschwerer Arbeit wird ein zweifacher Grundumsatz (PAL $=2)$ erreicht. Die Grenze zur Schwerstarbeit liegt beim dreifachen des Grundumsatzes (PAL = 3) und gilt für einen Acht-Stunden-Arbeitstag. Die Werte des Energieumsatzes sind sehr individuell und können Höchstwerte erreichen, die das Sechsfache des Grundumsatzes (PAL = 6) umfassen (Friedrich 2007).

Bereits durchgeführte Studien zum Sauerstoffverbrauch bei Forstwirten während der Holzernte (Kukkonen-Harjula \& Rauramaa 1984; Hagen et al. 1993) ergaben einen durchschnittlichen $\mathrm{VO}_{2}$-Wert von 1,8 1/ min, was bei einem $80 \mathrm{~kg}$ schweren Mann mit einem Grundumsatz von $280 \mathrm{ml} / \mathrm{min}$ einem PAL von 6,4 entspricht.

In Tabelle 1 werden Energieumsätze unter verschiedenen Bedingungen und zulässige Höchstwerte für berufliche Arbeit abgebildet.

Tabelle 1: Exemplarische Energieumsätze. ${ }^{7}$

\begin{tabular}{|l|c|c|c|c|}
\hline \hline \multicolumn{1}{|c|}{ Bedingung } & Energieumsatz in W & \multicolumn{2}{c|}{ VO in ml/ min bei einer männlichen Person } \\
\cline { 3 - 5 } & & $\mathbf{7 0 ~} \mathbf{~ k g}$ & $\mathbf{8 0} \mathbf{~ k g}$ & $\mathbf{9 0} \mathbf{~ k g}$ \\
\hline Grundumsatz & 85 & 245 & 280 & 315 \\
\hline Freizeitumsatz & 115 & 330 & 377 & 424 \\
\hline $\begin{array}{l}\text { Zulässige Höchstwerte für jahrelange } \\
\text { berufliche Arbeit, pro Tag }\end{array}$ & 240 & 690 & 788 & 887 \\
\hline $\begin{array}{l}\text { Zulässige Höchstwerte für jahrelange } \\
\text { berufliche Arbeit, pro Arbeitszeit }\end{array}$ & 490 & 1400 & 1600 & 1800 \\
\hline $\begin{array}{l}\text { Arbeitsumsatz bei Ausdauerleistungen } \\
\text { (Leistungssportler) }\end{array}$ & 1200 & 3400 & 3885 & 4371 \\
\hline \hline
\end{tabular}

\footnotetext{
${ }^{7}$ Angelehnt an Persson 2007.
} 
Körperlich anstrengende berufliche Arbeit, so auch das Berufsbild Forstwirt, wird mit einem PAL von 2,0-2,4 berechnet (FAO/ WHO/ UNU 2001; Gibala 2005; Oberriter 2006; Sluik et al. 2008; Krink \& Kolschewski 2011), so dass ein täglicher Energieverbrauch von bis zu $4500 \mathrm{kcal}$ erreicht wird (McArdle et al. 2000). Im Vergleich hierzu werden bei mittlerer Arbeit 2800-4000 kcal, bei sitzender Arbeit $2200 \mathrm{kcal}$ verbraucht (Atzler 1927; Åstrand 1987; Hollmann \& Hettinger 2000; Konopka 2009). Die nachfolgende Tabelle gibt eine Übersicht über bereits erhobene Werte zum Energieumsatz in der Forstwirtschaft. Bei der Betrachtung der zusammengetragenen Werte sollte das Alter der Quellen und die damit einhergehende Entwicklung der Arbeitsabläufe berücksichtigt werden.

Tabelle 2: Arbeitsenergieumsatz (kcal/ min) bei beruflichen Tätigkeiten in der Forstwirtschaft.

\begin{tabular}{|c|c|c|c|c|c|}
\hline Tätigkeit & $\begin{array}{l}\text { Spitzer- } \\
\text { Hettinger } \\
\text { (1969) }\end{array}$ & $\begin{array}{c}\text { Valentin } \\
(\mathbf{1 9 7 9})\end{array}$ & $\begin{array}{l}\text { Rost } \\
(2001)\end{array}$ & $\begin{array}{c}\text { FAO/ } \\
\text { WHO/UNU } \\
(2001) \\
\end{array}$ & $\begin{array}{c}\text { Kaminsky (1960) \& } \\
\text { Stentzel (1957) (zitiert } \\
\text { in Fleischer 2009) }\end{array}$ \\
\hline Gehen (ohne Last in der Ebene) & $2,1-5,0$ & 4,3 & 3,1 & & $3,2-4,3$ \\
\hline Steigen (ohne Last am Steilhang) & $7,2-14,4$ & & & & \\
\hline $\begin{array}{l}\text { Bergabgehen (ohne Last am Steil- } \\
\text { hang) }\end{array}$ & $2,8-9,4$ & & & & 5,3 \\
\hline Pflanzen in vorbereiteten Löchern & $2,2-3,3$ & & & 4,1 & \\
\hline Holzernte (Reine AZ) & & & & $5,4-8,0$ & $5,2-5,5$ \\
\hline Fällschnitt mit EMS & $4,3-6,5$ & & & 5,7 & $6,5-7,5$ \\
\hline Vermessen & & & & & 4,2 \\
\hline
\end{tabular}

Die aus der Tabelle ersichtlichen differierenden Energieumsätze bei ähnlichen Tätigkeiten unterstreichen die Individualität der Arbeitsabläufe und des Energiebedarfs und die Notwendigkeit, zur Ermittlung des Energieumsatzes individuelle Daten zu erheben. Trotz der individuellen Messung in der vorliegenden Studie sollte bei der Auswertung der Daten bedacht werden, dass die Messungen nur in einem kurzen Ausschnitt des Arbeitstages durchgeführt werden und dass ständig neue Situationen und Bedingungen in der Waldarbeit auftreten, auf die der Forstwirt sich einstellen muss und die er bewältigen muss. Aus diesem Grund kann es u. U. neben den interpersonellen Varianzen zu Varianzen im Energieumsatz kommen, die bei ein und derselben Person auftreten.

Bezüglich der Auswirkung körperlicher Aktivität auf die Gesundheit sollte bedacht werden, dass körperliche Aktivität im Beruf bei gleichzeitig fehlender Aktivität in der Freizeit allein für eine gesunde Lebensweise nicht ausreicht (Ilmarinen 1978). Die meisten Studien untersuchen heutzutage das Freizeitverhalten und dessen Auswirkung bei Berufsgruppen, die einer sitzenden Tätigkeit nachgehen. Hier konnte eine präventive und rehabilitative Wirkung regelmäßiger körperlicher Aktivität nachgewiesen werden (Lippke \& Vögele 2006; Biddle \& Mutrie 2008) mit einer positiven Beeinflussung der physischen als auch psychischen Gesund- 
heit (Hänsel 2007; Schlicht \& Brand 2007; Wagner \& Brehm 2008; Rudolph 2013). Darüber hinaus konnte ein Zusammenhang zwischen körperlicher Freizeitaktivität und Arbeitsqualität festgestellt werden (Pronk et al. 2004). Aus diesen Gründen sollte bei einer Erhebung der körperlichen Aktivität das Freizeitverhalten mit betrachtet werden.

\subsection{Weitere Einflussfaktoren}

Neben der körperlichen Aktivität gibt es weitere Faktoren, die den Energieumsatz beeinflussen. Zentrale und humorale Reaktionen wirken sich auf die Stoffwechselaktivität aus (Levine et al. 2005). Darüber hinaus kann ein Einfluss des Alters auf den Sauerstoffverbrauch verzeichnet werden (Maagh 2006). Bei einer Gruppe Forstwirte mit einem Altersdurchschnitt von 29 Jahren konnte bei motormanueller Holzernte ein $\mathrm{VO}_{2}$-Wert von $1,8 \mathrm{l} / \mathrm{min}(=49 \%$ von $\left.\mathrm{VO}_{2 \max }\right)$ gemessen werden. Hingegen wurde bei einem Altersdurchschnitt von 59 Jahren ein $\mathrm{VO}_{2}$-Wert von 1,5 1/ min (= $53 \%$ von $\mathrm{VO}_{2 \max }$ ) erhoben (Hagen et al. 1993). Zusätzliche Gewichte, das Schuhwerk und das Klima wirken sich ebenso auf den Energieumsatz aus (Esliger et al. 2005).

Eine Abhängigkeit vom Geschlecht konnte festgestellt werden, lässt sich jedoch mittels einer Umrechnung des Energieumsatzes auf die fettfreie Körpermasse (Körperkomposition) erklären (Åstrand 1987; Bitar et al. 1999). Eine geschlechtsspezifische Unterscheidung liegt nicht mehr vor, wenn der Energieumsatz auf die fettfreie Körpermasse (Körperkomposition) umgerechnet wird (Åstrand 1987).

Ein weiterer Aspekt für die Höhe des Energieumsatzes sind die Jahreszeiten. Es kann im Allgemeinen eine höhere körperliche Aktivität im Frühjahr festgestellt werden und eine geringere körperliche Aktivität im Herbst und Winter (Bitar et al. 1999; Santos et al. 2005; Ma et al. 2006). Diese Differenz bezieht sich bei Personen mit ausschließlich sitzender beruflicher Tätigkeit auf die unterschiedliche saisonabhängige Freizeitgestaltung. Auf Forstwirte bezogen konnten Kukkonen-Harjula und Rauramaa (1984) feststellen, dass bei der Holzernte im Herbst und im Winter gleiche Werte der Sauerstoffaufnahme erreicht werden. Neben der körperlichen Aktivität sollten somit die Jahreszeiten bei der Betrachtung des Energieumsatzes berücksichtigt werden, v. a. aus dem Grund, dass die Forstwirte verstärkt durch ihre unterschiedlichen Tätigkeiten ein saisonales Belastungsprofil aufweisen (Rudolph 2013).

\subsection{Wirkungsgrad und Respiratorischer Quotient}

Bei körperlicher Aktivität wird nur ein Teil des gesamten Energieumsatzes in eine mechanische Arbeit verwandelt. Dieses Verhältnis zwischen äußerer Arbeit und Gesamtumsatz in Prozent wird als mechanischer Wirkungsgrad (y) bezeichnet (Heck 1990; Hollmann \& Het- 
tinger 2000; de Marées 2003; Persson 2007; Kroidl et al. 2010) und mit der folgenden Formel berechnet:

$$
\mathrm{y}=\mathrm{A} \times 100 / \mathrm{E}-\mathrm{e} .
$$

Dabei ist A die verrichtete Arbeit (in kcal), E der Gesamtenergieumsatz (in kcal) und e der Ruheumsatz (in kcal) (Heck 1990; Hollmann \& Hettinger 2000). Bei dynamischer Arbeit mit großen Muskelgruppen, z. B. Ergometriearbeit, liegt der Wirkungsgrad zwischen 15 und $25 \%$ (Lehmann 1961c; Åstrand 1987; Hollmann \& Hettinger 2000; Persson 2007).

Zur Gewinnung der notwendigen Energie werden Fette, Proteine und Kohlenhydrate oxidiert (vgl. Kapitel 2). Der respiratorische Quotient (RQ) zeigt an, welche Nährstoffe oxidiert werden (Hall 2010). Der RQ ist ein dimensionsloser Quotient, „der das Verhältnis der pro Zeiteinheit abgegebenen Kohlendioxidmenge $\left(\mathrm{VCO}_{2}\right)$ zum aufgenommenen Sauerstoffvolumen $\left(\mathrm{VO}_{2}\right)$ angibt“" (Hollmann et al. 2006; vgl. auch Atzler 1927; Lehmann 1961c; Mellerowicz 1979; Rost \& Hollmann 1982; Åstrand 1987):

$$
\mathrm{RQ}=\mathrm{VCO}_{2} / \mathrm{VO}_{2}
$$

Anhand des RQ-Wertes, der die innere Atmung beschreibt (Atzler 1927), lässt sich feststellen, aus welchen Substraten die Energie bei der vorliegenden Belastung gewonnen wird. Bei einem RQ von 0,7 liegt eine reine Fettverbrennung vor. Ein RQ von 0,8 steht für Proteinverbrennung. Eine reine Kohlenhydratoxidation zur Energiegewinnung besteht bei einem RQ von 1,0 (Atzler 1927; Lehmann 1961c; Mellerowicz 1979; Åstrand 1987). Bei Hyperventilation kann ein RQ von > 1,0 erreicht werden, der durch die vermehrte Abatmung von $\mathrm{CO}_{2}$ erklärt werden kann (Mellerowicz 1979; Golenhofen 2006; Persson 2007).

Der RQ ist abhängig von der Intensität der Belastung und vom Zeitpunkt der letzten Nahrungsaufnahme (Manore 2004). Darüber hinaus bestimmen die Ernährung des Vortags, die Belastung am Vortag, der Zustand der Glykogenspeicher, die Speicherfähigkeit von Fettgewebe für $\mathrm{CO}_{2}$ und die Ventilation den RQ (Kroidl et al. 2010). Körperlich aktive Menschen gewinnen ihre Energie zu $60 \%$ aus der Oxidation komplexer Kohlenhydrate (McArdle et al. 2000).

In Kapitel 4 wird vor dem erarbeiteten theoretischen Hintergrund die Energiebilanz des Menschen unter Berücksichtigung verschiedener Faktoren erläutert. Besonderes Augenmerk wird dabei auf die Auswirkung der Ernährung und der körperlichen Aktivität auf die Energiebilanz gerichtet. 


\section{$4 \quad$ Energiebilanz}

Von einer Energiehomöostase spricht man, wenn die Energiezufuhr gleichzusetzen ist mit dem Energieverbrauch, der für den Grundumsatz, die Aufbereitung der Nahrung und körperliche und geistige Aktivität notwendig ist. Die Energienutzung kann nur nach einem oxidativen Abbau der Nahrungsstoffe zu $\mathrm{CO}_{2}$ und $\mathrm{H}_{2} \mathrm{O}$ und nach einer Transformation der Energie in Arbeit und Wärme stattfinden (Huth 2006). Die Energiebilanz wird definiert als:

$$
\mathrm{En}=\mathrm{Ea}+\mathrm{Ed},
$$

wobei En für den Energiegehalt der Nahrung, Ea für die vom Körper ausgegebene Energie und Ed für die Differenz des Energiegehalts des Körpers zwischen dem Beginn und dem Ende des beobachteten Zeitraums steht (Lehmann 1961c).

Eine Balance zwischen Energieverbrauch und -aufnahme findet beim menschlichen Organismus nicht pro Tag sondern in einem 7-Tage-Rhythmus statt (Kasper 2000). Das Verhältnis von Nahrungsaufnahme und Energieverbrauch hat Auswirkung auf das Körpergewicht und ist somit ein Spiegel von Energiebilanz und Trainingszustand (Tremblay \& Therrien 2006; Konopka 2009; Hall 2010). Bei einer Überernährung spricht man von einer positiven Energiebilanz, die einen Anstieg der Körpermasse durch Zunahme des Fettgewebes bewirkt (de Marées 2003; Friedrich 2007). Bei einer negativen Energiebilanz hingegen gewinnt der Organismus aus den Fettdepots und der Muskelmasse die notwendige Energie, so dass es zu einer Abnahme sowohl der Fettdepots als auch der Muskelmasse kommt (Manore 2004).

Um den hohen Energiebedarf bei gesteigerter körperlicher Aktivität zu decken, muss eine Nahrungszufuhr in ausreichender Menge gewährleistet sein. Eine Rolle spielt hierbei das Ernährungsverhalten, dass von Wuorinen (2014) wie folgt beschrieben wird: „People tend to eat the same volume of food each day, on days when they exercise, they will remain in an energy deficit“. Durch die Ernährungsgewohnheiten wird eine Balance folglich in eher längeren Zeiträumen hergestellt. Bei Dauerleistungen empfiehlt sich eine kohlenhydratreiche Kost, da eine erhöhte Glykogenkonzentration in der beanspruchten Muskulatur zu einer erhöhten Ausdauerbelastung führt (de Marées 2003). Eine fettangereicherte Kost sollte nur bei extrem hohem Energiebedarf zugeführt werden, da grundsätzlich eine fettarme Ernährung leistungsfördernd ist (Konopka 2009). Für Ausdauersportarten mit hohem Krafteinsatz wird eine kohlenhydratreiche, eiweißreiche und fettarme Kost empfohlen, wohingegen bei Sportarten mit azyklischem Bewegungsablauf und intervallartigen Belastungsspitzen die Auffüllung der Glykogenspeicher durch eine kohlenhydratreiche Ernährung im Fokus steht (ebd.). Auf eine ausreichende Zufuhr von Eiweiß mit hoher biologischer Wertigkeit sollte besonders bei Kraft- und Schnellkraftbelastungen geachtet werden (Biolo et al. 1995; Etheridge et al. 2008; Thompson 
et al. 2015). Allgemein gilt bei körperlicher Aktivität die Steigerung der Vitaminzufuhr als auch die Wasser- und Elektrolytzufuhr, um durch Schwitzen entstandene Verluste auszugleichen (de Marées 2003). Der durch körperliche Aktivität induzierte Mehrbedarf an Nährstoffen sollte so abgedeckt werden, dass die Körperkomposition konstant gehalten wird und keine Einschränkung der Leistungsfähigkeit und keine Beeinträchtigung der Gesundheit entstehen (König \& Berg 2010). An eine erhöhte Nahrungsaufnahme adaptiert sich der Grundumsatz, so dass Schwerstarbeiter einen täglichen Gesamtenergieverbrauch von $4500 \mathrm{kcal}$ erreichen (Lehmann 1961c; Konopka 2009).

Durch die Mechanisierung in der modernen Arbeitswelt reduziert sich die körperliche Belastung im Beruf. Kombiniert mit dem „Nahrungsüberangebot“ (Schusdziarra 2003) der Zivilisationskost (vgl. Kapitel 2) führen diese beiden Faktoren häufig zu einer positiven Energiebilanz. Um eine niedrigere Energiebilanz zu erlangen, ist es wichtig die Energiezufuhr zu senken bzw. den Nährstoffgehalt zu verbessern als auch den Energieumsatz durch körperliche Aktivität zu erhöhen (Åstrand 1987; Pfeiffer 2006). Da die Energiebilanz außerdem für die Auswirkung von körperlicher Aktivität auf die Fettoxidation relevant ist, sollte sie bei Interventionen zum Abbau der Fettdepots berücksichtigt werden (Melanson et al. 2009).

In den folgenden Kapiteln wird auf die Problematik Übergewicht als Folge einer positiven Energiebilanz eingegangen. Dabei wird zum einen der Fokus auf die Gründe und die Auswirkungen von Adipositas und zum anderen auf die Problematik der Klassifizierung von Adipositas gelegt.

\subsection{Adipositas}

Bei der Definition von Übergewicht wird das Körpergewicht ins Verhältnis zur Körpergröße gesetzt. Adipositas liegt vor, wenn der Anteil des Fettgewebes am Körpergewicht höher ist als normal (Pudel 1998). Adipositas ist immer die Folge einer langfristig positiven Energiebilanz, kann jedoch eine unterschiedliche Pathogenese haben (Mäueler 2006; Stensel 2008). Eine Gewichtszunahme kann einerseits durch konstante Nahrungsaufnahme und eingeschränkten Energieverbrauch, andererseits durch vermehrte Nahrungsaufnahme bei konstantem Energieverbrauch auftreten (Schusdziarra 2003).

Als Zivilisationskrankheit tituliert entsteht Adipositas durch die Anpassung an die durch Bewegungsarmut gekennzeichnete moderne Arbeitswelt und an die permanente Verfügbarkeit von Nahrungsmitteln mit hoher Energiedichte. Resultate dieser Anpassung sind körperliche Inaktivität bzw. Bewegungsmangel sowie eine Über- und Fehlernährung, die zu einer Erhöhung des Körpergewichts führen (Kasper 2000; Koh-Banerjee et al. 2003; de Marées 2003; 
Liebman et al. 2003; Golenhofen 2006; Oberriter 2006; Stensel 2008; Trembley \& Chaput 2008; Thiel 2011). Ein hoher Fettkonsum (Oberriter 2006), süße Getränke, große Portionen, geringe körperliche Aktivität und das Essen nebenbei (Liebman et al. 2003) sind spezifische Gründe für die Entstehung von Übergewicht. Des Weiteren konnte ein Zusammenhang zwischen Körperfettanteil und Bewegung festgestellt werden, so dass Levine et al. (2005) vermuten, dass Körperfett einen Faktor freisetzt, der körperliche Aktivität verringert. So entsteht ein Teufelskreis, in dem Bewegungsmangel kombiniert mit Über- bzw. Fehlernährung zu einem erhöhten Körperfettanteil führt, der wiederum die körperliche Aktivität herabsetzt. Übergewicht gilt als Indikator einer ungesunden Lebensweise. Die Zunahme an Körpergewicht gefährdet die Gesundheit und ist bei längerem Bestehen für Komorbiditäten verantwortlich (Hauner 1996; Schusdziarra 2003; Mäueler 2006; Tucker \& Gilliland 2007; Thiel 2011).

\begin{abstract}
„Wie bereits erwähnt spielt das Fettgewebe eine entscheidende Rolle im Intermediärstoffwechsel und ist an einer Reihe von Regulationsvorgängen des Körpers beteiligt. Diese Tatsache macht verständlich, dass eine Fehlfunktion des Fettgewebes (z. B. stark vermehrte Fettmasse (Adipositas)) vielfältige gesundheitliche Komplikationen bedingen kann.“" (Mäueler 2006).
\end{abstract}

Zu den durch Adipositas bedingten Krankheiten zählen Hypertonie, Hyperlipidämie, Diabetes mellitus, metabolisches Syndrom (Adipositas wird als wesentlicher pathogenetisch relevanter Faktor in 20-40 \% mit dem metabolischen Syndrom assoziiert) (Berg \& König 2005), degenerative Gelenkerkrankungen, Gallenerkrankungen, Atem- und Schlafstörungen, Venenleiden, bestimmte Karzinome (Pudel 1998; Hauner 1996). Des Weiteren können subjektive Beschwerden (z. B. Depressionen) als auch körperliche Beschwerden (z. B. Wirbelsäulen- und Kniegelenkbeschwerden) auftreten. Adipositas bildet zudem einen Risikofaktor für die Entwicklung von Herz- und Gefäßerkrankungen (Hauner 1996). Folge des Übergewichts ist somit eine höhere Anzahl an Arbeitsunfähigkeitstagen. Darüber hinaus konnte evaluiert werden, dass die Produktivität im Team bei übergewichtigen Menschen geringer ist (Pronk et al. 2004).

Die genannten Aspekte fordern sowohl präventive als auch therapeutische Maßnahmen für Adipositas. In den Vordergrund rücken hierbei die beiden Hauptfaktoren für Übergewicht: zum einen sollte körperliche Aktivität erhöht, zum anderen die Ernährung reduziert und umgestellt werden (di Pietro et al. 2004; Bowman \& Shanthy 2006; Tremblay \& Therrien 2006; Wareham 2007; Tremblay \& Chaput 2008; Thiel 2011). Bei der Nahrung sollte auf einen geringen Anteil an Fetten und süß schmeckenden Kohlenhydraten und zugleich auf eine ausreichende Zufuhr von hochwertigem Eiweiß, Vitaminen und Mineralien geachtet werden (de Marées 2003; Tremblay \& Chaput 2008). Neben den Dimensionen Bewegung und Ernäh- 
rung findet in aktuellen Maßnahmen Verhaltenstraining unter Einbeziehung des Umfelds Beachtung (Wirth 2008). Allein eine Erhöhung der alltäglichen Bewegung in Form von Treppe laufen statt Liftbenutzung oder laufen statt Bus-/ Autofahren ist laut Stensel (2008) genau so effektiv für den Gewichtsverlust wie aerobes Belastungstraining.

Eine Beurteilung des Körpergewichts sollte das Anforderungsprofil des Menschen berücksichtigen.

\footnotetext{
"A healthy weight is one that can be realistically maintained, allows for positive advances in exercise performance, minimizes the risk of injury or illness, is consistent with long-term good health, and reduces the risk factors for chronic disease."(ADA 2000; IOM 2002; zitiert in Manore 2004)
}

Hierauf lässt sich auch die These beziehen, dass fitte Übergewichtige ein vergleichbar geringeres Krankheitsrisiko haben als leistungsschwache Normalgewichtige (Warburton et al. 2006; zitiert in Thiel 2011). Die Problematik der Beurteilung des Körpergewichts, gerade bei Berufen mit körperlich schweren Tätigkeiten, wird im nächsten Kapitel thematisiert.

\subsection{Klassifizierung von Adipositas: Body Mass Index vs. Körperfettanteil}

Mittels der Erhebung der Daten zu Körpergröße und Körpermasse kann der Body Mass Index (= BMI) berechnet werden. Der BMI bezeichnet eine Messzahl zur Bestimmung des Normalgewichts, die sich aus dem Quotienten des Körpergewichts einer Person in Kilogramm und dem Quadrat der Körpergröße in Metern ergibt (Kasper 2000; Wirth 2003; Nowitzki-Grimm \& Grimm 2004; Thiel 2011). Laut WHO gilt bei Männern ein BMI zwischen 20 und 25 als normalgewichtig, Werte über 30 fallen in den Bereich von Adipositas (Roche Lexikon Medizin 1998; Kasper 2000; WHO 2000; de Marées 2003; Wirth 2003; Stensel 2008; Thiel 2011). Nach dieser Klassifizierung sind mehr als 19 \% der männlichen Bevölkerung in Deutschland adipös (Thiel 2011). Der BMI ist die aus wissenschaftlicher Sicht und laut WHO international anerkannte Angabe für Diagnose und Klassifikation des Übergewichts (Wenzel 2003; Oberriter 2006; Schauder \& Arends 2006).

Wird bei der Ermittlung des BMI das Körpergewicht ins Verhältnis zur Körpergröße gesetzt, wird noch keine Aussage über den Anteil des Fettgewebes am Körpergewicht erzielt. Z. B. verursacht eine große Muskelmasse bei gut trainierten Menschen einen hohen BMI, jedoch kann nicht auf Übergewicht geschlossen werden, da in diesem Fall der Körperfettanteil nicht erhöht ist (Semenick 1994b; Stone 1994b; Hollmann \& Hettinger 2000; de Marées 2003; Krink \& Kolschewski 2011; Platen 2012). Deshalb ist es notwendig, neben der Ermittlung des BMI zusätzlich eine Körperfettmessung durchzuführen, um eine bessere Bewertung des Körperbauzustandes und des Gesundheitsrisikos zu gewährleisten. 
Von den verschiedenen Methoden zur Messung der fettfreien Körpermasse hat sich die Kalipermetrie besonders bei Feldstudien bewährt (Parzikova 1974, zitiert in Hollmann \& Hettinger 2000; Herm 2003). Bei dieser anthropometrischen Methode handelt es sich um eine „metrische [...] Erfassung der Hautfaltendicke an verschiedenen Stellen des Körpers“ (Herm 2003). Mittels Messung mit einer speziellen Fettzange (= Kaliper) wird von der subkutanen Fettschichtdicke mehrerer Körperstellen auf die Körperfettmasse geschlossen (Tittel \& Wutscherk 1972; Wenzel 2003; Schauder \& Arends 2005). Da eine Zuordnung der gemessenen Werte zum prozentualen Körperfettanteil über komplizierte Formeln geschieht, kann bei dieser Messung nur von Schätzwerten gesprochen werden (Schusdziarra 2003; Schauder \& Arends 2006). Der Adipositas-Bereich liegt bei dieser Messmethode bei einem Körperfettanteil zwischen 20 und $25 \%$ (de Marées 2003).

Aufgrund der dargelegten Problematik befasst sich Kapitel 5 mit dem Anforderungsprofil von Forstwirten. Es soll ermittelt werden, inwieweit beim Berufsbild Forstwirt bzw. bei welchen Haupttätigkeiten von einer schweren körperlichen Arbeit gesprochen werden kann, ob saisonale Varianzen vorliegen und in welchem Umfang diese Aspekte eine kritische Betrachtung des BMI und des Körperfettanteils erfordern.

\section{$5 \quad$ Anforderungsprofil Forstwirt}

Forstwirte führen in ihrem Beruf unterschiedlichste Tätigkeiten durch. Aufgrund der großen Varianzen werden im Folgenden die Tätigkeiten analysiert und die daraus resultierenden Folgen aufgezeigt. Bei der Analyse sollen sowohl Belastung als auch Beanspruchung berücksichtigt werden. Belastung beschreibt dabei die Einflüsse auf den Arbeitenden, die aus der Art der Arbeitsaufgabe und deren Bedingungen resultieren. Unter Beanspruchung versteht man die Wirkung der Belastung auf den Arbeitenden. Hierbei werden sowohl Auswirkungen auf Organsysteme, auf das Belastungs- und Beanspruchungserleben und auf die Handlungsfähigkeit berücksichtigt (REFA-Verband für Arbeitsstudien und Betriebsorganisation 1991; Nowak 2003).

Waldarbeit zählt zu den Berufstätigkeiten mit dem höchsten Erkrankungsrisiko. „Trotz steigendem Maschineneinsatz ist ein großer Teil der Waldarbeit nach wie vor körperlicher Schwerarbeit und Witterungseinflüssen ausgesetzt.“ (Wirtschafts- und Forstverlag Euting KG 1996). Ursache hierfür sind Belastungen, die in unterschiedlichen Bereichen auftreten. Diese hohen körperlichen Beanspruchungen bilden die Grundlage für eine ausgeprägte körperliche Anstrengung und somit für einen hohen Energieumsatz während der Arbeit. Beeinflussende Faktoren sind hierfür die Bereiche Arbeitstätigkeiten, Witterungseinflüsse und Geländever- 
hältnisse. Die Arbeitsbedingungen, resultierend aus diesen Bereichen, ändern sich ständig. Ebenso spielen die Arbeitskraft und die handwerklichen Fähigkeiten der Forstwirte eine Rolle beim Ausführen der Tätigkeiten (ebd.; Stihl 1997; Sohns 2012).

Das Bestreben, im Sinne von Ergonomie ${ }^{8}$ und Arbeitssicherheit einen Arbeitsplatz optimal zu gestalten, ist im Bereich der Forstwirtschaft begrenzt, da der Forstwirt bei seiner Arbeit zahlreichen Umgebungseinflüssen ausgesetzt ist (Kuratorium für Waldarbeit und Forsttechnik 1999). Zwar nimmt ein Forstwirt verschiedene Körperhaltungen im Wechsel ein, jedoch sind ein möglichst körpernahes, beidarmiges Tragen von Lasten und Umsetzbewegungen ohne Rotation der Wirbelsäule (Nowak 2003) aufgrund der vorgegebenen Bewegungsabläufe nicht praktikabel. Aus diesem Grund ist es schwierig, ,das verwendete Arbeitsgerät und die Arbeit an die Fähigkeiten und Erfordernisse des Menschen anzupassen“ (Gilge et al. 2001). Aufgrund der äußeren Bedingungen lässt sich nur bedingt umsetzen bzw. erreichen, die Belastung im Beruf Forstwirt möglichst gering zu halten und gleichzeitig die Gesundheit und Leistungsfähigkeit zu erhalten (ebd.).

Die Vielfalt der auszuführenden Tätigkeiten erstreckt sich von Bestandsbegründung und -pflege über die Pflege und das Instandsetzen von Waldwegen bis hin zu Arbeiten im Jagdund Fischereibetrieb. Der wichtigste Aufgabenbereich findet sich allerdings in der Holzernte, die 40-50 \% der Arbeit eines Forstwirtes einnimmt (Gröger \& Lewark 2002). Die motormanuelle Holzernte zählt zu den sehr schweren körperlichen Arbeiten mit einem $\mathrm{VO}_{2}$-Wert $>$ 1,75 1/ min, was einer Leistung von ca. 125 Watt entspricht (Åstrand 1987; Sohns 2012).

Auffällig ist, dass in diesem Bereich drei Viertel aller Unfälle der Forstwirte stattfinden. Die nachfolgende Tabelle zeigt bei einer Auflistung der Arbeitsstunden in den unterschiedlichen Arbeitsbereichen einen noch höheren Anteil der Holzernte auf. Auch wenn die Anzahl der produktiven Arbeitsstunden im Jahr 2007 gegenüber 2006 um 96359 Stunden zurückgegangen ist, nimmt die Holzernte immer noch zwei Drittel der gesamten Arbeitsstunden ein.

Tabelle 3: Produktive Arbeitsstunden bei der Waldarbeit. ${ }^{9}$

\begin{tabular}{|l|r|r|r|}
\hline \hline Arbeitsbereich & $\begin{array}{l}\text { Produktive } \\
\text { Arbeitsstunden 2006 }\end{array}$ & $\begin{array}{l}\text { Produktive } \\
\text { Arbeitsstunden 2007 }\end{array}$ & $\begin{array}{l}\text { Veränderung } \\
\mathbf{2 0 0 7} \text { zu 2006 in \% }\end{array}$ \\
\hline Holzernte & 711.496 & 615.137 & $-13,5$ \\
\hline Holzbringung & 68.343 & 70.692 & $+3,4$ \\
\hline Waldverjüngung, Waldschutz & 153.967 & 151.891 & $-1,4$ \\
\hline
\end{tabular}

\footnotetext{
8 "Ergonomie (...) bezeichnet die Wissenschaft der menschengerechten Arbeitsgestaltung durch Anpassung der Arbeit und der Arbeitsgeräte an den Menschen." (Nowak 2003) Ziele der Ergonomie sind die Anpassung der Anforderungen der Arbeit an die Leistungsfähigkeit der Menschen (Vermeidung von Über- bzw. Unterbelastung), die Gestaltung von Maschinen etc., um eine fehler- und unfallfreie Bedienung zu ermöglichen, die Gestaltung der Arbeitsplätze, so dass natürliche Körperhaltungen gewährleistet werden und die Anpassung der Umweltbedingungen (ebd.).
}

\footnotetext{
${ }^{9}$ Ministerium für Ernährung und ländlicher Raum Baden Württemberg 2007.
} 
Witterungseinflüsse und Geländeverhältnisse wirken sich erschwerend auf die Arbeit der Forstwirte aus. Schlechte Arbeitshaltung und auch Stürze werden forciert durch ständig wechselnde äußere Bedingungen wie z. B. Hangneigung, Bodenbewuchs, Begehbarkeit und Witterungsverhältnisse (Stihl 1997; Gröger \& Lewark 2002). Diese Einflussfaktoren wirken sich ebenfalls auf den Energieumsatz der Forstwirte aus. So konnte bei Pflanzungen eine leichte Erhöhung der Hangneigung zu einem enormen Anstieg des Energieumsatzes führen (Jacke et al. 2013).

Des Weiteren ist ein Einfluss durch individuelle Leistungsfähigkeit vorhanden. Besonders beim Fällen ist die Produktivität stark abhängig von körperlichen und handwerklichen Eigenschaften des Forstwirts, zumal Leistungsschwankungen bei kurzen Arbeitsabläufen eher auftreten. Eine interindividuelle Leistungsstreuung nimmt somit mit wachsender Komplexität der Tätigkeiten zu (Reichel 1998), so dass bei der Untersuchung der Energiebilanz eine exakte individuelle Erhebung unterschiedlicher Daten notwendig ist.

Unterschieden werden muss bei der Forstarbeit zwischen der konventionellen und der hochmechanisierten. Ist die Wirbelsäule bei der konventionellen Arbeit im Wald besonders durch das Tragen und Heben schwerer Lasten, durch Zwangshaltungen und Arbeit in gebückter Körperstellung gekennzeichnet (Gröger \& Lewark 2002), so weist die mechanisierte bzw. motormanuelle Arbeit vermehrt statische Muskelarbeit auf. Zwar konnte durch die Einführung der Motorsäge die Effizienz der Waldarbeit gesteigert werden, jedoch nimmt dadurch die Belastung der Forstwirte nicht ab, sondern verlagert sich auf andere Ebenen (Stihl 1997). Gemeinsamkeiten lassen sich bei der konventionellen und der teilmechanisierten Waldarbeit in der Arbeit mit der Motorsäge finden. Ein Großteil der Waldarbeiter führt die Holzernte motormanuell aus (Gröger \& Lewark 2002). In Bezug auf die fehlende Dynamik im Arbeitsprozess ist festzuhalten, dass auch bei der konventionellen Waldarbeit statische Muskelarbeit geleistet wird, die sich im Zuge der fortschreitenden Technisierung vermehrt. Da die Waldarbeit heutzutage überwiegend teil- und hochmechanisiert organisiert ist (Gröger \& Lewark 2002), wächst die Bedeutung der statischen Muskelarbeit für das Berufsbild des Forstwirtes. Bei statischer Muskelbeanspruchung werden die Gefäße komprimiert und die Muskeldurchblutung vermindert. Ab einer Belastungsintensität von 50-60\% findet keine intramuskuläre Durchblutung statt, so dass es durch anaerobe Arbeit zu einem schnelleren Ermüdungseintritt kommt (Hollmann \& Hettinger 2000; Rost \& Graf 2001; Boutellier \& Ulmer 2005; Golenhofen 2006; Friedrich 2007; Persson 2007). Hohe Gewichte der Arbeitsgeräte und Arbeitsgegenstände, ein großer Anteil an Bückarbeit und unnatürlich verdrehte Arbeitshaltung auf- 
grund der Geländeverhältnisse addieren sich als weitere negative Belastungsformen bei der Forstwirtschaft (Gilge et al. 2001).

Obwohl Forstwirte durch die hohe körperliche Beanspruchung im Beruf eine hohe aerobe Kapazität aufweisen (Kurumatani et al. 1992), führen die aufgeführten Belastungen bei längerfristiger Ausführung zu einer Abnahme der Ermüdungstoleranz der Muskulatur. Die „Fähigkeit zur koordinierten, aktiven Führung der Wirbelsäule“ (Vollmer 1992) wird herabgesetzt, gleichzeitig erhöht sich jedoch die Gefahr unkoordinierter Bewegungen und somit auch das Verletzungsrisiko (Lilley et al. 2002). Die Ursachen sind der Waldarbeit z. T. immanent, jedoch auch anteilig in „objektiv vorhandenem oder subjektiv empfundenem Zeitdruck, Auslassen von Pausen und in körperlich und geistig ermüdenden, monotonen Arbeiten zu sehen“ (Vollmer 1992).

Die genannten Faktoren bedingen eine psychophysische stark ausgeprägte Belastung während der beruflichen Tätigkeit als Forstwirt (Stihl 1997; Yoshimura \& Acar 2004). Um dieser Belastung entgegenzuwirken, werden praktische Maßnahmen wie z. B. Arbeitsabwechslung, Maschinen-/ Geräte- und Werkzeugeinsatz, Pausengestaltung, richtige Körperhaltung (Motorsäge auf dem Oberschenkel abstützen, nah am Körper arbeiten) umgesetzt. Auch eine zweckmäßige Arbeitskleidung und eine persönliche Schutzausrüstung dienen dazu, die Gesundheit der Forstwirte zu schützen und deren Leistungsvermögen zu erhalten (Gilge et al. 2001). Dennoch lassen sich die hohen Beanspruchungsformen nur bedingt eindämmen. Die Möglichkeit, die körperliche Fitness durch körperlich anstrengende Arbeit zu verbessern (Straker \& Mathiassen 2009), trifft somit beim Berufsbild Forstwirt aufgrund der sehr hohen körperlichen Beanspruchung nicht zu.

Aufgrund der Verbindung zwischen physischen und psychosozialen Aspekten und muskuloskelettalen Erkrankungen sind Präventivprogramme im Forstbereich wünschenswert (Birger, Magnus \& Vetlesen 1998), die z. B. von den Niedersächsischen Landesforsten in den letzten Jahren in die Praxis umgesetzt werden (Rudolph 2013).

Berücksichtigt werden muss auch die Tatsache, dass es durch Mehrfachbelastungen häufig zu einer Überbeanspruchung und somit zu einer eingeschränkten Belastbarkeit im Waldarbeiterberuf kommt. Aus gesundheitlichen Gründen eingeschränkte Forstwirte können bei der Holzernte nicht mehr eingesetzt werden und führen „leichtere“ forstbetriebliche Aufgaben durch, so dass einsatzfähige Forstwirte die Holzernteeinsätze der Kollegen übernehmen müssen und somit eine Abwechslung mit anderen Tätigkeiten entfällt (Winterhoff 1991). Dies ist ein weiterer Grund, um präventive Maßnahmen im Betriebsbild zu verankern (Jones et al. 2013). 
Zusammenfassend lassen sich folgende Faktoren für steigende Anteile anspruchsvoller motormanueller Holzernte nennen:

1. Personalabbau

2. eingeschränkte Tätigkeitsbereiche von Teilen der Berufsgruppe

3. Hochmechanisierung in weniger anspruchsvollen, Teilmechanisierung vermehrt in sehr anspruchsvollen Geländeverhältnissen möglich

4. Holzmarkt mit Forderung nach mehr Kontinuität in der Belieferung (führt zu ausgedehnter Holzerntesaison).

Resultat der weiterhin bestehenden Belastung ist ein vermehrter Energiebedarf während der Ausübung der Arbeiten (vgl. Kapitel 3.1). Zu berücksichtigen ist auch, dass die Forstwirte stark differierende Belastungssituationen bewältigen. Eine Unterscheidung zwischen motormanueller und maschineller Holzernte, zwischen Pflanzungen, Waldpflege und Gehen (mit einer $6 \mathrm{~kg}$ schweren Motorsäge) muss bei der Betrachtung und bei der Messung des Energieverbrauchs beachtet werden. Zusätzlich beeinflussen Geländeverhältnisse den Energieverbrauch. Im Bergland ist das Einsetzen von Maschinen eingeschränkt, so dass zu erwarten ist, dass im Flachland weniger Energie verbraucht wird. Darüber hinaus wird die Produktivität (bzw. IST-Leistung) von der Erfahrung, der Motivation, der Leistungsfähigkeit und der Geschicklichkeit des einzelnen Forstwirts beeinflusst (Sohns 2012). Interindividuelle Unterschiede, die in Abhängigkeit zu Trainingszustand und leistungsrelevanten Persönlichkeitsmerkmalen stehen, wirken sich auf die Leistungsfähigkeit aus (Boutellier \& Ulmer 2005). Somit ist eine Messung des Energieverbrauchs in unterschiedlichen Anforderungssituationen notwendig, deren Ergebnisse individuell unter Berücksichtigung der o. g. Einflüsse betrachtet werden sollten.

\subsection{Motorische Stereotype}

Aus einseitigen Belastungen im Beruf des Forstwirts entstehen muskuläre Dysbalancen, die Haltungsschäden implizieren (Laube \& Hildebrandt 2000; Röwekamp 2002). Haltung und Bewegung sind multifaktoriell bedingt, determiniert durch die Körperstatik, die Muskelfunktion (Verspannungen, Abschwächungen, Inkoordination), die Flexibilität (Hyper-, Hypomobilität), durch psychische Faktoren und durch Sensomotorik (Alltagsstereotypen) (Bittmann \& Badtke 1994).

Der Forstwirt ist bei der Ausführung seiner beruflichen Aufgaben zu einer Fehlbelastung gezwungen (vgl. Kapitel 5), aus der sich unökonomische Bewegungsmuster entwickeln. Anzustreben ist jedoch eine realitätsnahe Haltung, die funktionelle Asymmetrien berücksichtigt. 
Koordination spielt für die Haltung und für die Ausführung von Bewegungen eine große Rolle, da der Körper immer bestrebt ist, ökonomisch zu arbeiten bzw. den Energieverbrauch gering zu halten (Bittmann \& Badtke 1994; Sparrow et al. 2000). Durch funktionelles Training wird ein Bewegungsablauf optimiert, neu erlernte Bewegungsmuster werden gespeichert, so dass unökonomische Stereotype durchbrochen und korrigiert werden und es aufgrund des besseren Wirkungsgrades der Muskulatur, der besseren Stoffwechselökonomie und der besseren Koordination und Technik zu einem reduzierten Sauerstoff- und Energiebedarf kommt (Lehmann 1961b; Mellerowicz 1979; Bittmann \& Badtke 1994; Conrooy \& Earle 1994; Hollmann \& Hettinger 2000; O’Dwyer \& Neilson 2000; Graf \& Rost 2001; Hollmann et al. 2006; Friedrich 2007; Persson 2007; Konopka 2009; Platen 2012). So vermuten auch Jacke et al. (2013) bei der Bewertung des Energieumsatzes eines Forstwirtes bei unterschiedlichen Pflanzverfahren einen Einfluss des Übungsgrades einer Technik auf den notwendigen Energieverbrauch.

Bei einem koordinativen Training sollte auf eine zweckmäßige Beanspruchung geachtet werden, um gezielte und funktionelle Trainingsreize zu setzen (Dudley \& Harris 1994; Boyle 2010). Für die Umsetzung von Haltungsregulation und zielgerichteter Bewegungsausführung ist eine ständige sensorische Kontrolle erforderlich, die auf Informationen verschiedener Wahrnehmungssysteme basiert (Dudley \& Harris 1994; Kondziella 2003; Streicher 2005; Haas et al. 2007; Hübscher 2011).

Problematisch ist das Training ökonomischer Stereotype bei der Optimierung beruflicher Tätigkeiten. Vorgegebene Arbeitsgeräte und äußere Bedingungen wie Geländeverhältnisse, Bodenbewuchs oder Gefahrensituationen bei der Forstarbeit lassen sich nicht an eine ökonomischere Arbeitsausführung adaptieren. Eine ideale Haltung lässt sich nur bedingt einnehmen, so dass statische Belastungen im Berufsbild Forstwirt nicht vermeidbar sind und somit der Energieverbrauch durch die z. T. statisch schlecht ausgeglichene Haltung höher ist. Aufgrund der meist statischen Beanspruchungsform bei körperlicher Schwerstarbeit, die durch ungünstige klimatische Bedingungen intensiviert wird, wird im Vergleich zu Leistungssportlern im Ausdauerbereich eine geringere aerobe Kapazität erreicht (Hollmann et al. 2006).

Dadurch, dass die von statischer Muskelarbeit geprägten Tätigkeiten einen ganzen Arbeitstag durchgeführt werden, erfordert die Arbeit der Forstwirte jedoch isometrische Kraft im Ausdauerbereich. Die Kraftkomponente rückt auch bei der Ausführung schwerer Arbeit in den Hintergrund, sensorische und motorische Leistung sind ausschlaggebend, um die stützenden Strukturen in möglichst optimalen Positionen zu halten (Bittmann \& Badtke 1994; Schlumberger \& Eder 2001; Streicher 2005; Hübscher 2011). Für das Training sowohl von isometri- 
scher Kraft als auch von Ausdauer nennen Hollmann \& Hettinger (2000) das Schnelligkeitstraining als verbindende Position, um gleichzeitig für beide Komponenten Trainingsreize zu setzen. Grundsätzlich gilt, motorische Stereotype funktionell zu trainieren mit einer größtmöglichen Orientierung an der Zielbewegung (Boyle 2010).

Je größer die Ökonomie einer Bewegung ist, umso geringer ist der benötigte Energieumsatz und umso geringer ist das subjektive Anstrengungsempfinden (Lehmann 1961a/ b). Durch das Einüben motorischer Stereotype und die neuromuskuläre Adaptation wird die Bewegungsausführung optimiert, Bewegungen können kontrollierter ausgeführt werden, so dass es zu einer „gleichgewichtsregulatorischen Alltagskompetenz“ (de Marées 2003) kommt, durch die Stürze oder Unfälle mit einhergehenden Verletzungen verringert werden können.

Im folgenden Kapitel werden die Tätigkeiten beschrieben, die ein Forstwirt hauptsächlich durchführt und die bei der vorliegenden Erhebung gemessen wurden. Aus den Bewegungsbeschreibungen lässt sich ableiten, ob und in welcher Form sich motorische Stereotype durch die Belastung entwickeln und wie diese sich auf die Haltung auswirken.

\subsection{Tätigkeitsbeschreibungen bei der Waldarbeit}

In den folgenden Kapiteln werden unterschiedliche Tätigkeiten im Beruf Forstwirt beschrieben und analysiert. Es findet eine Betrachtung der Tätigkeiten statt, die bei der Datenerhebung dieser Studie durchgeführt wurden. Eine Bewegungsbeschreibung ist wichtig für die Betrachtung der Energiebilanz, da Bewegung und körperliche Aktivität einen großen Einfluss auf den Energieverbrauch haben (Montoye et al. 1996, vgl. Kapitel 3). Mittels der biomechanischen Beschreibung bzw. der beschreibenden Bewegungsanalyse werden Bewegungen erklärt und die Belastungssituation betrachtet (Roth \& Willimczik 1999; Olivier \& Rockmann 2003).

Darüber hinaus muss berücksichtigt werden, dass eine detaillierte und komplette Bewegungsbeschreibung nicht möglich ist, da die Arbeiten im Feld durchgeführt werden und somit keine standardisierten Bedingungen geschaffen werden können.

\subsubsection{Holzernte}

Motormanuelle Holzernte nimmt den zeitlich umfangreichsten Arbeitsbereich im Beruf Forstwirt ein. Sie wird mit einer ca. 7,5 kg schweren Motorsäge durchgeführt und ist als körperlich schwer einzustufen und wird von äußeren Bedingungen beeinflusst. Während der reinen Arbeitszeit liegt ein Energieverbrauch von 5,2-5,5 kcal/ min vor (Stentzel 1957 und Kaminsky 1960, zitiert in Fleischer 2009). Dieser Energieverbrauch bezieht sich jedoch ausschließlich auf die Reine Arbeitszeit und nicht auf den gesamten Tag, so dass die Allgemeine 
Arbeitszeit bei der Bewertung des Energieverbrauchs des Arbeitstages berücksichtigt werden muss.

Auswirkung auf die Durchführbarkeit der motormanuellen Holzernte haben die Witterungsverhältnisse. Bei langanhaltenden Nässeperioden, extremen Schneelagen und plötzlichem Tauwetter kann die Holzernte entweder nicht durchgeführt werden oder die Arbeit wird erschwert bzw. die Leistung wird gemindert. Auf die Produktivität wirken sich außerdem Wege- und Rückezeiten aus (Sohns 2012). Ein weiterer wichtiger Aspekt ist die Geländeform, die den möglichen Mechanisierungsgrad vorgibt und eine erhöhte Anforderung an den Forstwirt stellt. Holzernte in Steillagen bedingt eine größere Anzahl an Stürzen, eine höhere Verletzungsgefahr und eine höhere Beanspruchung (Raab et al. 2002; LWF 2004; Sohns 2012). Allein beim Gehen am Steilhang mit Werkzeug werden von Forstwirten Spitzenwerte für den Gesamtumsatz erreicht (Fleischer 2009).

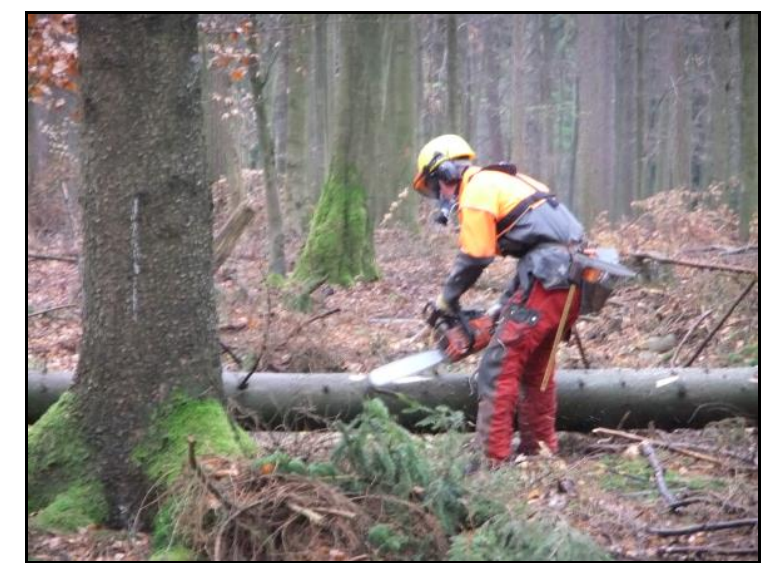

Abbildung 2: Forstwirt bei motormanueller Holzernte.

Das Arbeitsverfahren als Solches unterteilt sich in verschiedene Ablaufabschnitte. Diese sind das Aufsuchen des Baumes, der Fällvorgang, Entasten und Zopfen bei gleichzeitiger Längenvermessung, Wenden, Restentasten und buchmäßige Erfassung des aufgearbeiteten Holzes (Raab et al. 2002; Kieser 2009; Sohns 2012). Beim Fällvorgang und bei der Aufarbeitung muss der Forstwirt Zwangshaltungen einnehmen, so dass die erforderliche statische Muskelarbeit häufig in flektierter Wirbelsäulenhaltung erfolgt. Des Weiteren verlangt der unebene Waldboden und das Klettern über Stamm und Äste eine erhöhte Aufmerksamkeit und einen erhöhten Anspruch an koordinative Fähigkeiten.

\subsubsection{Sammelhieb}

Beim Sammelhieb handelt es sich ebenfalls um eine Arbeit mit der Motorsäge. Bäume, die durch Wind oder andere Ursachen umgestürzt sind, werden aufgearbeitet. 


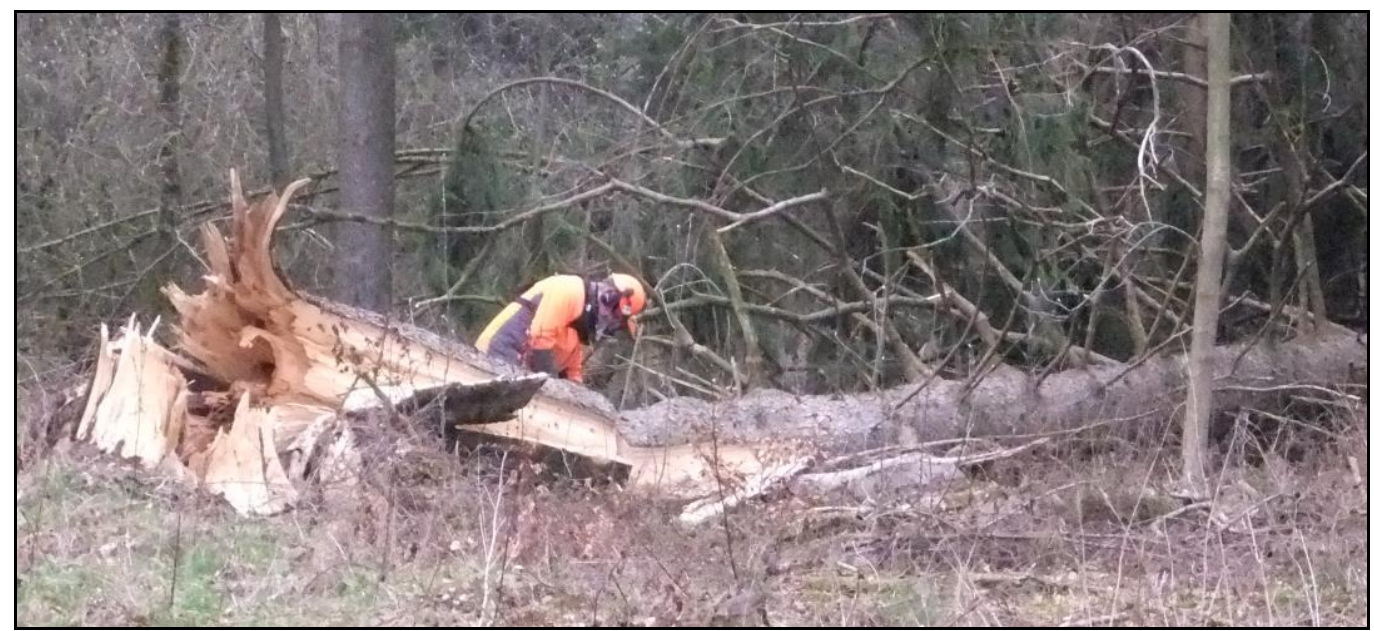

Abbildung 3: Forstwirt beim Sammelhieb.

Die Körperhaltung und Belastung sind zu vergleichen mit dem Profil der motormanuellen Holzernte, wobei unter erschwerenden (Baumstämme liegen übereinander, herrschende Spannungen im Holz) bzw. erleichternden Faktoren (längere Wege, kein Fällschnitt) gearbeitet wird. Auf unebenem Boden mit z. T. starkem Bodenbewuchs wird die Arbeit mit der Säge durchgeführt. Eine besondere Gefahr verbirgt sich beim Sammelhieb im Arbeitsgegenstand Holz. Durch die Position der Stämme und Äste und ggf. der Wurzelteller entstehen Spannungen im Holz, die bei der Aufarbeitung freigesetzt werden können (Stihl 1997).

\subsubsection{Jungwuchs-/ Jungbestandspflege}

Jungwuchs-/ Jungbestandspflege bzw. Läuterung umfasst Maßnahmen, die mit minimalen Eingriffen zur Mischungs- und Begleitwuchsregulierung den angestrebten Waldentwicklungstyp ermöglichen (Wenzel \& Stratmann 2006). Ziel dieser Maßnahmen ist die Sicherung der Qualität, Stabilität und Vitalität in Pflanzungen und Naturverjüngungen bis zum Beginn der Durchforstung (Raab 1981; Ministerium Ländlicher Raum Baden-Württemberg 1997; Wenzel \& Stratmann 2006; Rothkegel et al. 2012). Es wird zwischen manuellen und motormanuellen Arbeitsverfahren unterschieden. Arbeitsgeräte sind Handarbeitsgeräte (z. B. Sense, Schere, Heppe) und leichte Motorsägen und Freischneidegeräte. Eine persönliche Schutzausrüstung ist je nach Arbeitsverfahren zu wählen (Raab 1981; Ministerium Ländlicher Raum BadenWürttemberg 1997; Wenzel \& Stratmann 2006). Die Arbeitsmittelauswahl ist vom Brusthöhendurchmesser (BHD) der Bäume und von der weiteren Verwertung des Holzes abhängig (Feist 2012). In den vorliegenden Untersuchungen wurde die motormanuelle Jungwuchspflege mit der leichten Motorsäge und mit dem Freischneider durchgeführt. 


\subsubsection{Jungwuchspflege mit der Motorsäge}

Die motormanuelle Jungwuchspflege wird bei einem stärkeren Durchmesserbereich der Pflanzen (ab 7-8 cm) eingesetzt (Raab 1981; Wenzel \& Stratmann 2006). Gearbeitet wird mit leichteren, ca. $5 \mathrm{~kg}$ schweren Motorsägen.

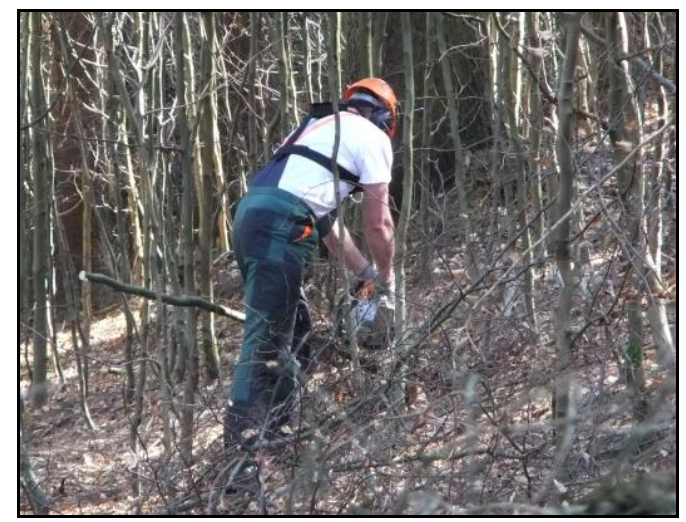

Abbildung 4: Forstwirt bei der Jungwuchspflege mit der leichten Motorsäge.

Das standardmäßige Fällen der Entnahmebäume findet mit Schrägschnitt oder Fallkerb statt. Anschließend werden die Bäume grob entastet, um nach dem Fällen den Bodenkontakt leichter herzustellen, der für die Verrottung notwendig ist. Der Forstwirt fällt den Baum auf Brusthöhe, um anschließend den stehen gebliebenen Stumpf bodennah abzusägen. Der abgesägte Kronenteil wird per Hand heruntergezogen. Ist dies nicht möglich, wird der Hänger vom Forstwirt per Klappschnitt zu Fall gebracht bzw. mehrfach gekappt, so dass der gesamte Kronenteil Kontakt zum Boden hat und verrotten kann. Diesen Vorgang bezeichnet man als ökologisches Ablegen (Ministerium Ländlicher Raum Baden-Württemberg 1997; Wenzel \& Stratmann 2006). Je nach Bestand und situativen Entscheidungen führt der Forstwirt die Arbeiten in gebückter und damit belastender Haltung durch. Die Jungwuchspflege wird in der Literatur als beliebt, jedoch ergonomisch belastend und gefährlich für den Forstwirt beschrieben (Feist 2012).

\subsubsection{Jungwuchspflege mit dem Freischneider}

Bis zu einer Bestandeshöhe von ca. 2,5 m wird die motormanuelle Jungwuchspflege mit Freischneidern ausgeführt. Ein Wurzelstockdurchmesser von 3-7 cm indiziert den Einsatz von Freischneidern, der vielfach in nicht differenzierten, überdichten Naturverjüngungen angebracht ist (Raab 1981; Ministerium Ländlicher Raum Baden-Württemberg 1997; Wenzel \& Stratmann 2006). 


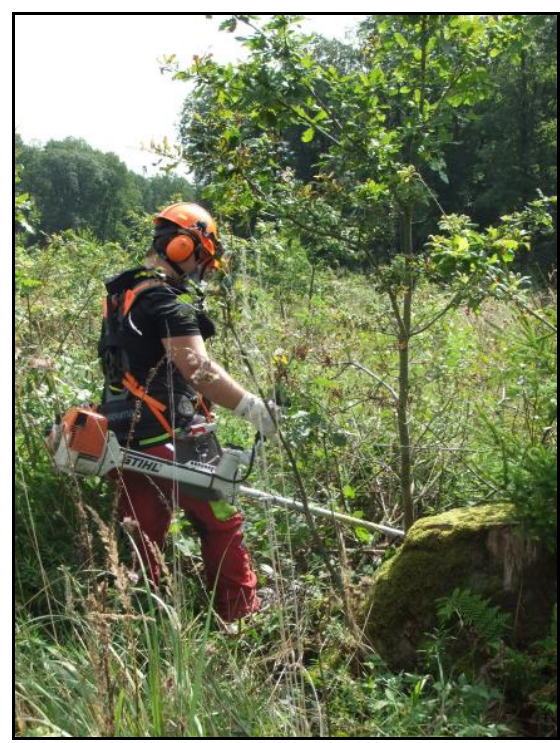

Abbildung 5: Forstwirt bei der Jungwuchspflege mit dem Freischneider.

Die Arbeit mit dem Freischneider wird durch verstellbare Tragegurte und breite Führungslenker erleichtert. Damit wird versucht, das Gewicht gleichmäßig zu verteilen und eine ergonomische Haltearbeit zu ermöglichen. Durch den meist starken Bodenbewuchs wird dem Forstwirt die Handhabung erschwert, so dass durch das Arbeiten im unwegsamen Gelände häufig Rotationsbewegungen mit gleichzeitigem Kraftaufwand in der Wirbelsäule stattfinden.

\subsubsection{Pflanzungen}

Im Frühjahr ist das Aufgabenfeld der Forstwirte von Pflanzungen geprägt, welches auch als schwere Arbeit bezeichnet werden kann (Sullmann \& Byers 2000). Jedoch ist eine andere Belastung als bei der Arbeit mit der Motorsäge vorzufinden (Rudolph 2013). Es gibt unterschiedliche Pflanzverfahren, weshalb im Folgenden lediglich die Pflanzverfahren mittels einer Bewegungsanalyse beschrieben werden, die bei der Datenerhebung dieser Studie gemessen wurden.

Beim Rhodener Pflanzverfahren handelt es sich um eine Spaltpflanzung, die mit einer speziellen Haue ausgeführt wird (Jacke \& Zimmer 2011). In einer Tasche führt der Forstwirt je nach Pflanzenart und Pflanzengröße 25-50 Pflanzen mit sich. 

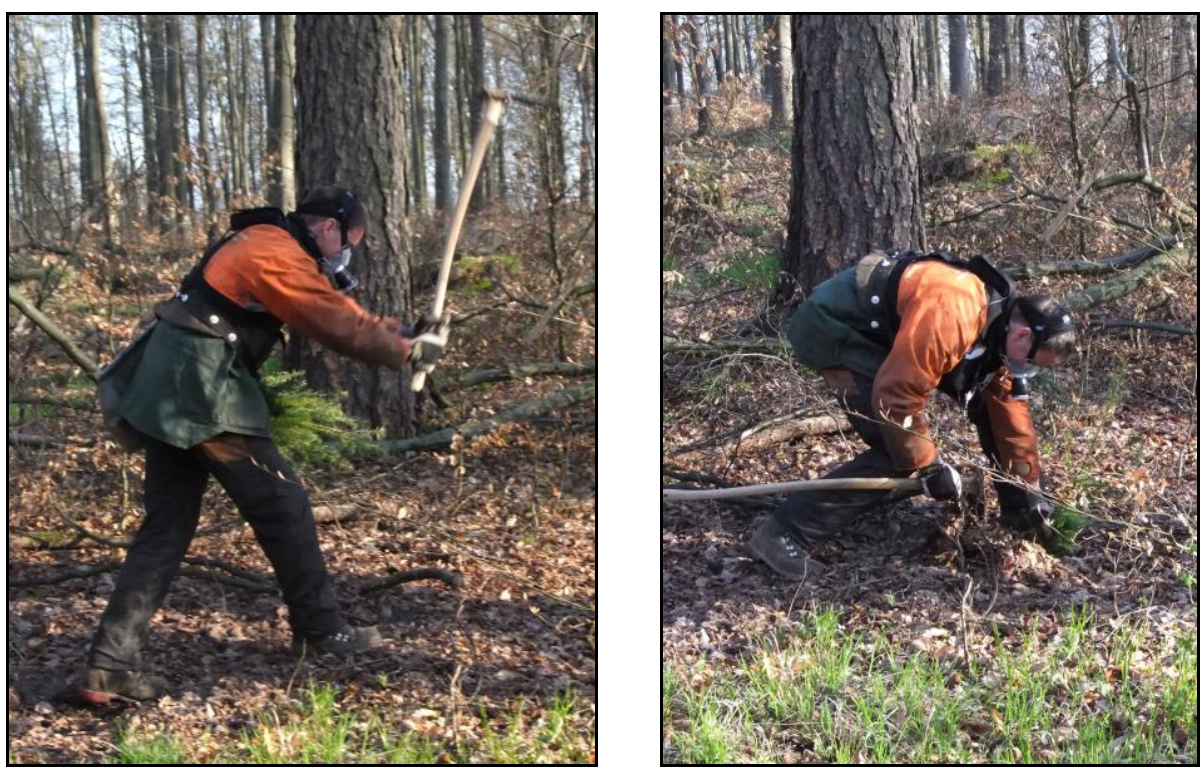

Abbildung 6: Rhodener Pflanzverfahren.

Die Haue wird beim Schlag mit beiden Händen geführt, wobei der Griff beim Aufschlagen gelockert wird, um die Krafteinwirkung auf die Hände zu verringern. Um das Erdreich zu lockern, wird die Haue nach vorne angehebelt. Durch das Herunterdrücken des Stiels bis auf die Erde wird der Pflanzspalt geöffnet und die Pflanze kann entlang des Blattes in den Pflanzspalt geschoben werden. Abschließend wird der Pflanzspalt mit dem Hackenstich hinter die Pflanze und durch Anhebeln des Stiels geschlossen und festgetreten (Scherer 1996; Versuchsund Lehrbetrieb für Waldarbeit und Forsttechnik 1996). Das Rhodener Pflanzverfahren hat einen hohen Anteil an gebückter Arbeitshaltung und somit eine hohe Belastung des muskuloskelettalen Systems (Spiegelhalter 2012; Jacke 2013).

Das Harzer Pflanzverfahren zählt zu den T-Pflanzungen. Es wird bei starkem Bodenbewuchs oder stark durchwurzelten Böden angewendet. Wie beim Rhodener Verfahren trägt der Forstwirt eine Tasche mit 25-50 Pflanzen.
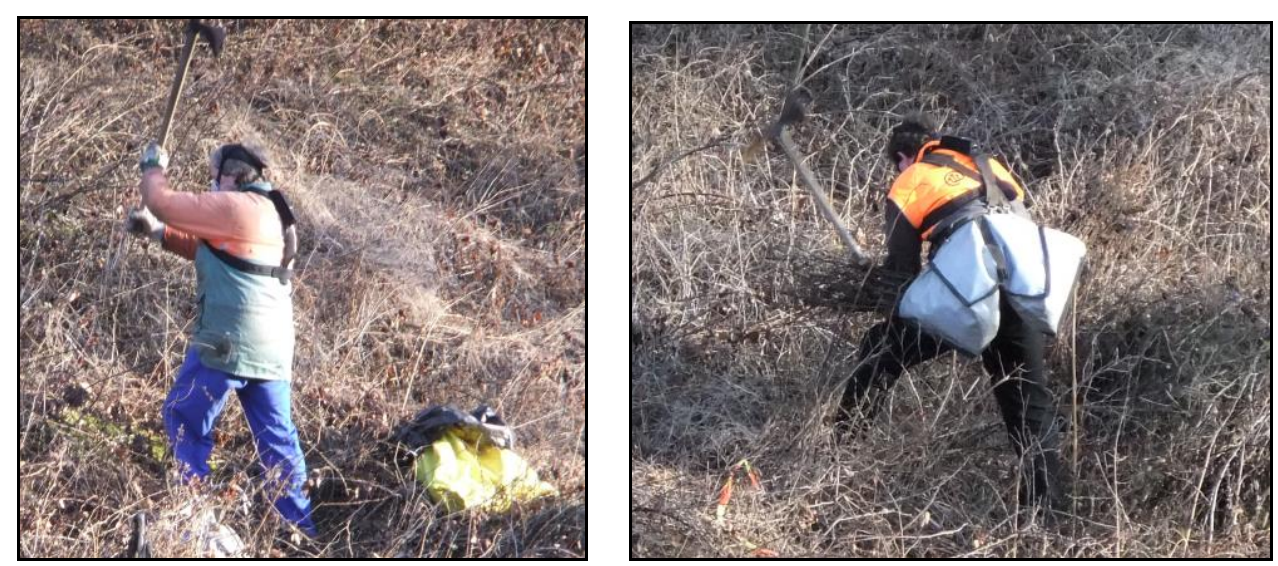

Abbildung 7: Harzer Pflanzverfahren. 
Die Pflanzhaue wird beidhändig geführt und es werden zwei Hiebe ausgeführt, die senkrecht zueinander stehen und ein „T“ bilden. Nachdem der Boden durch Anhebeln nach vorne gelockert wurde und der Pflanzspalt durch das Hinunterführen des Stiels geöffnet wurde, wird die Pflanze in den Pflanzenspalt eingeführt. Die Haue wird in der Form aus dem Boden gezogen, dass die Pflanze senkrecht steht. Durch ein leichtes Ziehen an der Pflanze werden die Wurzeln ausgerichtet bevor die Pflanze angetreten wird (Jirikowski 2010; Versuchs und Lehrbetrieb für Waldarbeit und Forsttechnik 1996). Das Winkelpflanzverfahren hat einen hohen Anteil an gebückter Haltung und somit durch die höhere körperliche Belastung einen erhöhten Energiebedarf (Küster \& Bombosch 1993; Jacke \& Zimmer 2011; Spiegelhalter 2012; Jacke 2013). Motormanuelle Pflanzverfahren werden mit einem Einmann-Erdbohrgerät durchgeführt. Durch das Aufforsten mit Pflanzlochbohrer ist das ergonomische Setzen größerer Pflanzen möglich. Der Bohrer wird dabei von einem Forstwirt über die Fläche geschoben und beim Herstellen eines Pflanzloches in den Boden geführt. In diesem arbeitsteiligen Verfahren bohrt ein Forstwirt die Löcher, ein anderer setzt die Pflanzen in die Löcher und schließt den Boden lagenweise, um eine Stauchung der Wurzeln zu vermeiden (Jirikowski 2010; Versuchs- und Lehrbetrieb für Waldarbeit und Forsttechnik 1996).

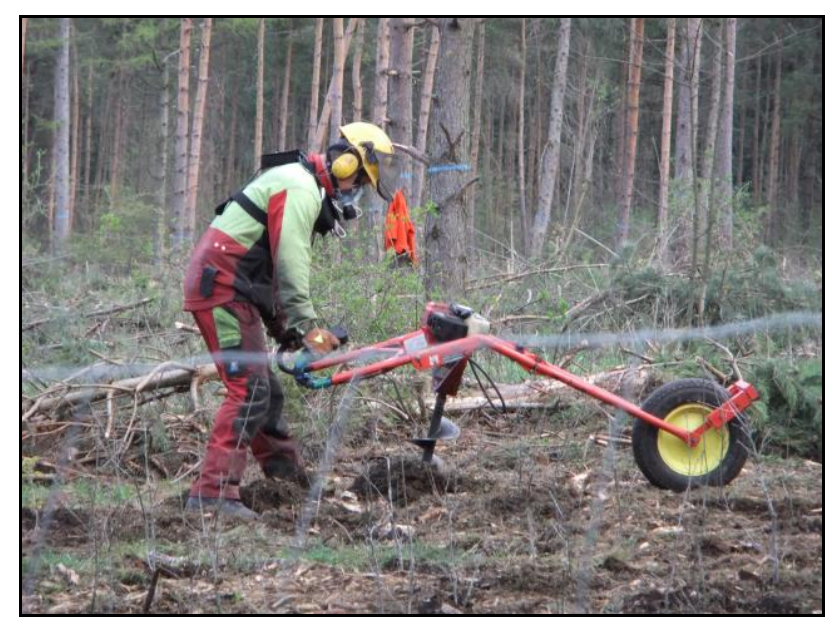

Abbildung 8: Aufforstung mit Pflanzlochbohrer.

Bei der Erhebung in der vorliegenden Studie wurden die Forstwirte beim Bedienen des Pflanzlochbohrers und nicht beim Setzen der Pflanzen gemessen. Beim Bohren der Löcher neigt der Forstwirt seinen Oberkörper nach vorne und drückt den Bohrer in den Boden. Dabei wird die Wirbelsäule unter Anspannung der Flexoren vermehrt in eine flektierte Position gebracht, wobei die Arme die Kraftübertragung auf den Bohrer ausüben. 


\subsubsection{Bau von Geländern}

Ein Aufgabenbereich der Forstwirte ist die Wegepflege. Um nicht mit den Zeiten der Holzernte und der Pflanzungen zu kollidieren, wird diese Arbeit meist in den Sommermonaten ausgeführt. Der Bau von Geländern ist aus versicherungstechnischen Gründen notwendig, um bei Überquerungen mit Gräben die Sicherheit der Passanten zu gewährleisten. Eine Teilaufgabe hierbei ist das Gießen von Fundamenten.
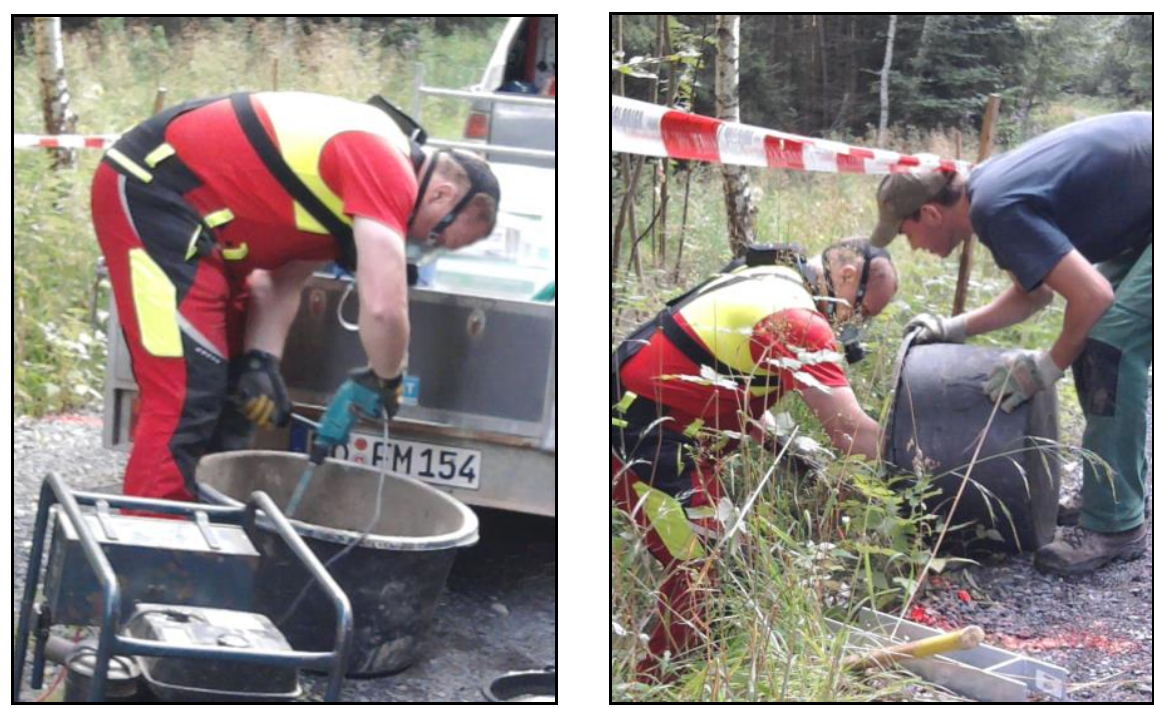

Abbildung 9: Forstwirte beim Gießen eines Fundaments.

Bei der gemessenen Tätigkeit im Zusammenhang mit der vorliegenden Studie sind die Grabungen für die Fundamente bereits durchgeführt. Der Zement wird mit einem Bohrmaschinenquirl angerührt, wobei der Behälter auf dem Waldboden positioniert ist und der Forstwirt in flektierter Haltung den Zement anrührt. Nach dem Transport des Zements werden die Fundamente gegossen und fixiert. Auch hierbei arbeitet der Forstwirt überwiegend in flektierter Haltung, um die Ständer bedarfsgerecht im Fundament zu befestigen.

Nachdem im allgemeinen Teil (Kapitel I) die theoretischen Grundlagen und der aktuelle Forschungsstand dargestellt und auf das Berufsbild Forstwirt bezogen wurden, befasst sich Kapitel II mit dem empirischen Teil der Arbeit. Neben dem Studiendesign werden die Ergebnisse der Untersuchung dargestellt, die im anschließenden Kapitel III diskutiert werden. 


\section{EMPIRISCHER TEIL}

In diesem Teil der Arbeit werden zunächst das Untersuchungsdesign und die Erhebungsinstrumente beschrieben. Daran anschließend werden die Ergebnisse dargestellt und unter Berücksichtigung des aktuellen Forschungsstandes diskutiert. Aufgrund des integrierten Hypothesendesigns werden die Hypothesen in der Ergebnisdarstellung geprüft.

\section{Studiendesign}

In der Untersuchung werden quantitative Erhebungsmethoden (Kalipermetrie, indirekte Kalorimetrie, Fahrradspiroergometrie) mit qualitativen Informationen verbunden. Die standardisierten Ernährungs- und Aktivitätsprotokolle liefern zum einen messbare Daten, die jedoch zum anderen durch qualitative Angaben ergänzt werden. Ergebnisse bereits durchgeführter Erhebungen mit ähnlichem Studiendesign unterstreichen die Angemessenheit einer solchen Verknüpfung, um das multifaktorielle Geschehen Energiebilanz zu untersuchen (Montoye et al. 1996; Schutz et al. 2001; Ma et al. 2006; Silva et al. 2011).

Der Erhebungszeitraum für den einzelnen Probanden dauert je eine Woche zu drei Messzeitpunkten. Im Dezember 2011, Januar und Februar 2012 während der Holzernte liegt t1. Die Erhebung zu t2 findet im Frühjahr 2012 (März bis Mai) statt, wobei Pflanzungen und Sammelhieb gemessen wurden. In den Sommermonaten 2012 (Juli bis September) liegt t3. Zu diesem Messzeitpunkt wurden Wegepflege, Jungwuchspflege und Holzernte durchgeführt. Die Messzeitpunkte wurden gewählt, um die jahreszeitliche Differenz bei den forstwirtschaftlichen Tätigkeiten zu berücksichtigen.

Während der Woche der Erhebung führen die Probanden die Ernährungs- und Aktivitätsprotokolle. Die humanphysiologischen Messungen erfolgen für die Probanden an jeweils einem Tag während ihrer beruflichen Tätigkeiten, der direkt vor oder nach der Woche der Protokollierung liegt, so dass gewährleistet ist, dass die Probanden in der protokollierten Woche die Tätigkeiten durchgeführt haben, bei der sie gemessen wurden. Aufgrund des Datenschutzes werden die Daten pseudonymisiert. 


\subsection{Hypothesen}

Basierend auf dem theoretischen Hintergrund lassen sich für die Untersuchung der Energiebilanz folgende Hypothesen aufstellen:

H1: Der Energieumsatz der Forstwirte unterscheidet sich zu den unterschiedlichen Jahreszeiten und dadurch unterschiedlichen Tätigkeiten.

Das Anforderungsprofil der Forstwirte weist unterschiedliche Tätigkeiten auf. Trotz der hohen körperlichen Beanspruchung besteht ein erhöhter BMI, der in den Bereich des Übergewichts einzuordnen ist. Daher lässt sich vermuten, dass der Energieumsatz bei den unterschiedlichen Tätigkeiten differiert und somit eine über das Jahr gesehene positive Energiebilanz entsteht.

H2: Das Ernährungsverhalten der Forstwirte unterscheidet sich signifikant zu den unterschiedlichen Wochentagen (Arbeitstage vs. Wochenende).

Die Tendenz, dass am Wochenende eine höhere Energieaufnahme vorliegt als an Werktagen, wird in einigen Studien nachgewiesen (vgl. Kapitel 2). Durch die hohe körperliche Aktivität beim Berufsbild Forstwirt scheint diese Tendenz stärker ausgeprägt zu sein, da der hohe Energieumsatz an Werktagen ggf. nicht durch Energieaufnahme ausgeglichen werden kann und somit eine vermehrte Energieaufnahme am Wochenende notwendig ist.

\section{H3: Die Energieaufnahme im Winter ist kalorienreicher als im Frühjahr und im Sommer.}

Zwar haben bisherige Studien keine eindeutigen Ergebnisse bzgl. der Differenz der Energieaufnahme zu unterschiedlichen Jahreszeiten ergeben, jedoch lässt sich aufgrund der erhöhten körperlichen Aktivität der Forstwirte vermuten, dass der erhöhte Energieumsatz durch die berufliche Tätigkeit durch eine vermehrte Energieaufnahme ausgeglichen wird. Wie in Kapitel 5 beschrieben, variieren die Tätigkeiten bei der Waldarbeit. Der durch die höhere körperliche Beanspruchung im Beruf entstehende höhere Energieumsatz impliziert möglicherweise eine höhere Energieaufnahme in den Wintermonaten.

H4: Die Energiebilanz korreliert negativ mit dem Energieumsatz bei der beruflichen Aktivität.

Der Energieumsatz ist ein Faktor, der die Energiebilanz bedingt, weshalb eine negative Korrelation zwischen diesen beiden Items zu erwarten ist. Mit dieser Hypothese soll jedoch geprüft werden, ob ein erhöhter Energieumsatz eine erhöhte Energieaufnahme bedingt. Bei hoher 
körperlicher Beanspruchung stellt sich der Körper auf die Bedürfnisse des Körpers ein und das Essverhalten wird entsprechend angepasst, so dass eine erhöhte Energieaufnahme den erhöhten Energieumsatz ausgleicht. In diesem Fall wäre die Hypothese falsifiziert. Es lässt sich die Vermutung anstellen, dass die Forstwirte bei saisonal wechselnden Tätigkeiten und daraus resultierenden variierenden Energieumsätzen ihr Essverhalten nicht an die körperliche Beanspruchung anpassen.

\subsection{Probandenrekrutierung}

Zur Probandenrekrutierung nehmen alle Forstwirte der Forstämter Münden, Reinhausen und Seesen der Niedersächsischen Landesforsten an einer Informationsveranstaltung teil. Während der Veranstaltung werden das Ziel der Studie, der Ablauf und die Erhebungsinstrumente vermittelt. Interessierte Forstwirte werden nachfolgend schriftlich über die Untersuchung informiert (Anhang 1) und nehmen nach Unterschreiben der Einverständniserklärung (Anhang 2) an der Studie teil.

Die teilnehmenden Forstwirte arbeiten somit alle in Südniedersachsen. Bei der Bewertung der Ergebnisse sollte daher auf zwei Aspekte geachtet werden. Zum einen sind die Anfahrtswege zum Arbeitsort der Forstwirte relativ gering. Umfassen die Reviere im Norden Niedersachsens größere Flächen, so fallen für die untersuchten Forstwirte im Süden kürzere Wege im Revier an, so dass die Reine Arbeitszeit einen größeren Anteil einnimmt. Zum anderen gibt es in den Revieren sowohl ebene Flächen als auch Flächen mit Hangneigung. Die Forstwirte werden also je nach Arbeitsgebiet bei ihrer Arbeit von der Hangneigung und vom Bodenbewuchs beeinflusst, was bei der Auswertung des Energieumsatzes bei beruflicher Tätigkeit berücksichtigt werden muss.

\subsection{Erhebungsinstrumente}

Im Folgenden werden die einzelnen Messmethoden zur Erhebung der unterschiedlichen Parameter beschrieben. Dabei kommt es zu einer Verknüpfung physiologischer Erhebungsmethoden und klassischer Arbeitsstudien, um die Arbeit der Forstwirte und deren Anstrengungsgrad klassifizieren zu können. Um die komplexe Fragestellung nach der Energiebilanz möglichst exakt bearbeiten zu können, empfiehlt sich eine solche Methodenverknüpfung (Tommlinson \& Manenica 1977; Liebman et al. 2003; Ma et al. 2005; Ma et al. 2006; Peterson et al. 2007). Die Vor- und Nachteile einzelner Erhebungsmethoden wurden im Voraus abgewogen. Da alle Methoden zur Untersuchung des Energieverbrauchs Fehler aufweisen, ist der Entscheidungsparameter für die Begründung der Methoden die Anwendbarkeit im Feld (Montoye et al. 1996). 


\subsubsection{Soziodemografische und anthropometrische Daten}

In einer Abfragung werden Daten zum Geburtsjahr, Erkrankungen, Medikamenteneinnahme, Dauer der beruflichen Tätigkeit als Forstwirt und zur Schulbildung erfasst. Der Einfluss von Bildungsstand und sozialem Kontext begründet die Erhebung dieser Faktoren, um einige von vielen vermuteten Einflüssen zu berücksichtigen (Peterson et al. 2007). Darüber hinaus wird nach dem Nikotinkonsum gefragt, da bei Rauchern der Stoffwechselumsatz in Ruhe und bei Belastung zunimmt und Raucher aufgrund ihres erhöhten Glukosebedarfs bei submaximalen Belastungsstufen schneller ermüden (Huie 1996; Hollmann \& Hettinger 2000).

Unter Anthropometrie versteht man die Vermessung des Menschen (Schauder \& Arends 2006). Trotz der bereits diskutierten Problematik des BMI und der Vollständigkeit halber werden außerdem Körpergröße und Körpermasse gemessen. Um vergleichbare Werte zu erlangen wird die Messung mit einer kalibrierten Waage und möglichst wenig Kleidung durchgeführt (Semenick 1994b). Die internationale Anerkennung des Wertes begründet die Erhebung, zumal somit die Möglichkeit des Vergleichs von BMI und Körperfettanteil möglich ist. Sowohl die nachgewiesene Zuverlässigkeit der Körperfettbestimmung mittels Kalipermetrie als auch die praktikable Anwendbarkeit bei Felduntersuchungen begründen die Wahl der Methode zur Körperdepotfettbestimmung (Parizkova 1974, zitiert in Hollmann Hettinger 2000; Åstrand 1987; Semenick 1994b; Herm 2003; de Marées 2003). Die Messung mit Präzisionskaliper bestimmt die Körperkomposition, indem das subkutane Fettgewebe gemessen wird. Um eine umfassende Beurteilung des Körperfettanteils vollziehen zu können, werden zehn Körperstellen markiert (Brožek \& Keys 1951; vgl. Anhang 3). Ein geübter Tester greift mit Zeigefinger und Daumen die Hautfalte und misst 1-2 Sekunden nach dem Greifen ca. $1 \mathrm{~cm}$ neben der Greifstelle mit dem Präzisionskaliper die Dicke der Hautfalte. Der Kaliper übt dabei einen konstanten Druck von $10 \mathrm{~g} / \mathrm{mm}^{2}$ aus (Tittel \& Wutscherk 1972; Semenick 1994b; Schusdziarra 2003; Schauder \& Arends 2006; Platen 2012).

Die Messung an den zehn Körperstellen wird drei Mal durchgeführt, so dass für jede Hautfalte ein Mittelwert aus drei Werten gebildet wird. Abschließend wird der Mittelwert aller zehn Körperstellen errechnet, so dass in den Tabellen nach Parizkova (1974, zitiert in Hollmann \& Hettinger 2000) die Gesamtkörperfettmasse abgelesen werden kann. Die Tabelle wird mittels komplexer Formeln erstellt, so dass es sich bei den mittels Kalipermetrie erhobenen Werten um Schätzwerte handelt, die auch als solche in der Auswertung betrachtet werden müssen (Tittel \& Wutscherk 1972; Schusdziarra 2006; Schauder \& Arends 2006).

Die Kalipermetrie ist somit wichtig für die Beurteilung des BMI, um die Körperkomposition besonders bei Athleten, Kraftsportlern oder Arbeitern mit einer hohen körperlichen Beanspru- 
chung zu bestimmen. Darüber hinaus steigt bei einem geringen Körperfettanteil die maximale $\mathrm{O}_{2}$-Aufnahme pro kg Körpergewicht (Stone 1994b; Hollmann \& Hettinger 2000; Krink \& Kolschewski 2011).

\subsubsection{Ernährungsprotokoll}

Ernährungsprotokolle bilden die Basis für eine Ernährungsanamnese und somit für eine präventive und therapeutische Ernährungsberatung (Kohnharst \& Ollenschläger 2003). Die Vielfalt in den Ernährungsgewohnheiten verhindert jedoch eine ,exakte und absolut reproduzierbare Messung“ (Schneider \& Heseker 2006). So konnten Cai et al. (2004) z. B. eine höhere personale als interpersonale Variation in der Energieaufnahme feststellen.

Zur Erfassung der Zahl der Mahlzeiten, der zeitlichen Verteilung der Mahlzeiten sowohl über den Tag als auch über die Woche, der Menge und der Zusammensetzung der Nahrung führen die Probanden ein 7-Tage-Protokoll. Das Drei-Tage-Schätz-Protokoll (Lührmann 1999) wird auf eine Woche erweitert, um einerseits die Energie- und Nährstoffzufuhr jeweils an Arbeitstagen und an freien Tagen zu erheben und andererseits eventuelle Differenzen zwischen jahreszeitbedingten Tätigkeiten und Essgewohnheiten zu erfassen (Hartman et al. 1990; Schusdziarra 2003; Westerterp et al. 2005; Schauer \& Arends 2006; Schneider \& Heseker 2006). So stellte z. B. McCarthy (2014) fest, dass die Qualität der Ernährung am Wochenende durch vermehrte Fett- und Alkoholzufuhr und eine erhöhte Energieaufnahme geringer ist.

Mit Hilfe eines Verzehrprotokollheftes wird den Probanden die Dokumentation erleichtert (Anhang 4). Ziel ist es, den Aufwand für das Wiege- bzw. Schätzprotokoll für die Probanden möglichst gering zu halten, um eine höhere Kooperationsbereitschaft zu erlangen. Aus diesem Grund beträgt der Erhebungszeitraum nur eine Woche, da bei einer längeren Protokollphase die Angaben unvollständiger würden und auf einfache Gerichte umgestiegen würde, so dass die Validität abnähme (Schneider \& Heseker 2006). Dennoch sollte ,the influence of underreporting“ (Bellisle 2014) bei der Auswertung der Ernährungsprotokolle bedacht werden.

Die Auswertung der Ernährungsprotokolle erfolgt mittels der Ernährungssoftware DGE-PC, welche den Bundeslebensmittelschlüssel (BLS) beinhaltet und die D-A-CH-Referenzwerte ${ }^{10}$ zugrunde legt. Durch die computergestützte Protokollauswertung wird eine Ernährungs- und Nährstoffanalyse durchgeführt (Faustin \& Ellrott 2006), so dass die o. g. Faktoren erhoben und ausgewertet werden können.

\footnotetext{
${ }^{10}$ D-A-CH-Referenzwerte sind Referenzwerte zur Nährstoffzufuhr, die von Deutschland (D), Österreich (A) und der Schweiz $(\mathrm{CH})$ gemeinsam festgelegt wurden (Deutsche Gesellschaft für Ernährung; Österreichische Gesellschaft für Ernährung; Schweizerische Gesellschaft für Ernährungsforschung; Schweizerische Vereinigung für Ernährung 2012).
} 


\subsubsection{Aktivitätsprotokoll}

Die Messung von Aktivität und Bewegung eines Menschen über den Zeitraum einer Woche gestaltet sich schwierig. Der Zeitraum einer Woche ist notwendig, da sich eine Balance im Energiehaushalt nicht pro Tag sondern meist in 7-Tage-Perioden einstellt (Kasper 2000). Die exakte Messung des Energieverbrauchs ist über einen längeren Zeitraum nicht möglich, so dass mittels indirekter Kalorimetrie lediglich einzelne Arbeitselemente gemessen werden. Es handelt sich somit bei der Bestimmung des Energieverbrauchs um Schätzwerte, da es keine Methode gibt, um exakte Werte über einen längeren Zeitraum zu erheben (Kreymann 2006; Laquale 2007).

In zahlreichen Studien wurden verschiedene Messmethoden getestet (Montoye et al. 1996; Schutz et al. 2001; Zhang et al. 2003; Esliger et al. 2005; Grand et al. 2006; Barreira et al. 2009; Spierer \& Hagins 2011; van Hees et al. 2013). Da bisher keine Methode alle Aspekte körperlicher Aktivität berücksichtigt, empfiehlt es sich, ergänzende Methoden anzuwenden und die Wahl der Messinstrumente an das Untersuchungsobjekt anzupassen (Schutz et al. 2001; van Hees et al. 2013).

Aktivitätsprotokolle bieten eine gute Möglichkeit, eine möglichst genaue Erhebung der körperlichen Tätigkeiten über eine Woche zu erfassen (Ilmarinen 1978; Montoye et al. 1996; McArdle et al. 2000; Santos et al. 2005; Manore et al. 2009; Silva et al. 2011). Vorteilhaft an dieser Methode sind die geringen Kosten und die parallele Durchführbarkeit der Protokollierung mehrerer Probanden, ohne dass ein Beobachter anwesend sein muss (Montoye et al. 1996). Hierzu ist jedoch eine gute Kooperation der Probanden notwendig, weshalb die Forstwirte vorab mit der Methodik vertraut gemacht wurden und ihnen die wichtigsten Aspekte der Protokollierung verdeutlicht wurden. Nachteilig sind evtl. auftretendes ungenaues Protokollieren und unexaktes Abschätzen der Intensität der Belastung (ebd.). Diesen Problemen wurde versucht entgegenzuwirken, indem die Aktivitätsprotokolle tabellenförmig angelegt wurden, so dass die Probanden in 15-minütigen Zeitintervallen die jeweils ausgeführte Tätigkeit ankreuzen (Anhang 5). Die angegebenen Tätigkeiten im beruflichen Kontext wurden an das Berufsbild Forstwirt angepasst, um den Probanden die Zuordnung ihrer Tätigkeiten zu erleichtern.

Der Energieumsatz der einzelnen Tätigkeiten wird über MET (vgl. Kapitel 3.1 und 6.3.3) geschätzt. Um bei der vorliegenden Studie mit exakteren Werten arbeiten zu können, wurde der Energieumsatz aller Probanden zu jedem Messzeitpunkt bei der Haupttätigkeit zum jeweiligen Zeitpunkt mittels indirekter Kalorimetrie gemessen. Bei den Tätigkeiten, die nicht gemessen wurden, wurde der MET-Wert auf der Grundlage von Tabellen (Ainsworth et al. 1993, 
2000 \& 2011; vgl. Tabelle 4) geschätzt, die eine Abschätzung des Energieverbrauchs für alltägliche als auch spezifische Aktivitäten beinhalten und eine gängige Methode bei der Auswertung von Aktivitätsprotokollen sind (Montoye et al. 1996). Grundlage dieser Schätzung bildet der mit Hilfe der Mifflin-Formel berechnete Grundumsatz der Forstwirte (vgl. Kapitel 3). 
Tabelle 4: MET-Werte für körperliche Aktivitäten bei Forstwirten. ${ }^{11}$

\begin{tabular}{|c|c|c|c|c|}
\hline \multicolumn{4}{|c|}{ Tätigkeit } & MET \\
\hline \multicolumn{4}{|c|}{ *Schlaf- und Ruhezeiten } & 0,9 \\
\hline \multicolumn{5}{|c|}{ * Aktivitäten während der Berufstätigkeit } \\
\hline \multirow[t]{2}{*}{ Sitzen } & \multicolumn{3}{|c|}{ Leichte Arbeit (z. B. Schreibtischarbeiten, PC-Arbeit) } & 1,5 \\
\hline & \multicolumn{3}{|c|}{ Mittelschwere Arbeit (z. B. Maschine fahren, Auto fahren) } & 2,5 \\
\hline \multirow[t]{3}{*}{ Stehen } & \multicolumn{3}{|c|}{ Leichte Arbeit } & 3,0 \\
\hline & \multicolumn{3}{|c|}{ Mittelschwere Arbeit (z. B. Pflanzungen) } & 4,5 \\
\hline & \multicolumn{3}{|c|}{ Schwere Arbeit (z. B. motormanuelle Holzernte) } & 8,0 \\
\hline \multirow[t]{4}{*}{ Gehen } & \multicolumn{3}{|c|}{ Langsam } & 3,5 \\
\hline & \multicolumn{3}{|c|}{ Zügig/ schnell } & 4,0 \\
\hline & \multicolumn{3}{|c|}{ Etwas tragend (z. B. Tasche) } & 3,5 \\
\hline & \multicolumn{3}{|c|}{ Etwas Schweres tragend (ca. $10 \mathrm{~kg}$ ) } & 3,8 \\
\hline \multirow{8}{*}{\multicolumn{2}{|c|}{$\begin{array}{l}\text { Weg zur/ von } \\
\text { Arbeit }\end{array}$}} & \multicolumn{2}{|c|}{\begin{tabular}{l|l} 
Gehen & Langsam
\end{tabular}} & 3,5 \\
\hline & & & Zügig/ schnell & 4,3 \\
\hline & & \multirow[t]{4}{*}{ Radfahren } & Langsam $(<15 \mathrm{~km} / \mathrm{h})$ & 3,5 \\
\hline & & & Mäßig (15-20 km/h) & 5,8 \\
\hline & & & Schnell $(20-23 \mathrm{~km} / \mathrm{h})$ & 6,8 \\
\hline & & & Sehr schnell (23-26 km/h) & 9,0 \\
\hline & & \multicolumn{2}{|c|}{ Auto fahren, aktiv } & 2,0 \\
\hline & & \multicolumn{2}{|c|}{ Bus, Bahn, Auto fahren, passiv } & 1,3 \\
\hline \multicolumn{5}{|c|}{ Nicht angeführte Tätigkeiten: } \\
\hline \multicolumn{5}{|c|}{ * Aktivitäten außerhalb der Berufstätigkeit } \\
\hline \multicolumn{4}{|c|}{ Tätigkeiten im Sitzen (z. B. Essen, Lesen, Fernsehen, Telefonieren, Auto fahren) } & 1,3 \\
\hline \multicolumn{4}{|c|}{ Tätigkeiten im Stehen (z. B. Körperpflege), langsames Gehen $(<4 \mathrm{~km} / \mathrm{h})$, Shopping } & 2,2 \\
\hline \multicolumn{4}{|c|}{ Leichte Hausarbeit (z. B. Kehren, Staub wischen, Kochen, Bügeln), Musizieren } & 2,8 \\
\hline \multicolumn{4}{|c|}{ Lebensmittel einkaufen, Betreuung von Kindern, zügiges Gehen (4-6 km/h) } & 2,5 \\
\hline \multicolumn{4}{|c|}{ Mittelschwere Haus- und Gartenarbeit (z. B. Fenster putzen, Rasen mähen) } & 3,5 \\
\hline Reparat & $\operatorname{arbei}$ & en, handwer & itigkeiten (z. B. Fliesenlegen, Malerarbeiten) & 3,3 \\
\hline Körperl & $\mathrm{sch}$ & vere Haus- $u$ & narbeit (z. B. Umgraben, Schnee schaufeln) & 4,3 \\
\hline Sehr sch & ere $\mathrm{I}$ & aus- und Ga & t (z. B. Holz hacken, Tragen schwerer Lasten) & 6,0 \\
\hline Nicht an & efühı & e Tätigkeite & & \\
\hline * Sport & he $A$ & ktivitäten & & \\
\hline Fit im F & & & & 5,0 \\
\hline Gymnas & $\mathrm{K}, \mathrm{lar}$ & gsames Radf & eiten, Tischtennis, Trainertätigkeit, Volleyball & 4,1 \\
\hline Golf, $\mathrm{Ta}$ & en & & & 4,9 \\
\hline Aerobic & 3ask & ball, mäßig & aren, Wandern & 6,2 \\
\hline Badmin & n, In & ne Skating, & Skifahren, Tennis & 5,4 \\
\hline Bergstei & en, $F$ & ßball, Handl & sing, schnelles Radfahren, Schwimmen & 6,8 \\
\hline Judo, se & schr & elles Radfah & & 8,9 \\
\hline Nicht an & fühı & e Sportarten & & \\
\hline
\end{tabular}

Der Energieumsatz bei der Haupttätigkeit der Forstwirte zum jeweiligen Messzeitpunkt wird jedoch mittels indirekter Kalorimetrie gemessen (vgl. Kapitel 6.3.5). Bei dieser Messung des Energieumsatzes sollte berücksichtigt werden, dass sich die Gesamtarbeitszeit in die Reine

\footnotetext{
${ }^{11}$ Angelehnt an Ainsworth 2011.
} 
Arbeitszeit (RAZ) und in die Allgemeinen Zeiten (AZ) unterteilt. Unter der RAZ versteht man die Zeit, die ausschließlich durch die betreffende Tätigkeit verursacht werden. Die Allgemeinen Zeiten beinhalten Rüst-, Verteil- und Erholungszeiten und werden meist in Prozenten angegeben. In Rüstzeiten wird das Arbeitssystem für die Arbeitsaufgabe vorbereitet bzw. nachbereitet (z. B. Werkzeug bereitlegen, Motorsäge auftanken). Verteilzeiten umfassen alle Arbeitsabläufe, die erforderlich sind, um einen Arbeitsablauf planmäßig auszuführen (z. B. Werkzeug nachschärfen, Anweisungen durch den Revierleiter einholen, Kleidung richten). Erholungszeiten dienen der Unterbrechung der Tätigkeit, um aufgetretene Ermüdung abzubauen (Lehmann \& Schmidtke 1961; Backhaus 1980; REFA 1991).

Die folgende Tabelle gibt eine Übersicht über die prozentuale Aufteilung von RAZ und AZ bei den gemessenen forstwirtschaftlichen Tätigkeiten (Kuratorium für Waldarbeit und Forsttechnik 2000; REFA-Fachausschuss Forstwirtschaft 2004):

Tabelle 5: Allgemeine Zeit und Reine Arbeitszeit für forstwirtschaftliche Tätigkeiten. ${ }^{12}$

\begin{tabular}{|l|c|c|}
\hline \hline \multicolumn{1}{|c|}{ Tätigkeit } & $\begin{array}{c}\text { Allgemeine Zeit (AZ) } \\
\text { in \% }\end{array}$ & $\begin{array}{c}\text { Reine Arbeitszeit (RAZ) } \\
\text { in \% }\end{array}$ \\
\hline Rhodener Pflanzverfahren & 20 & 80 \\
\hline Winkelpflanzung & 20 & 60 \\
\hline Einsatz von handgeführten Erdbohrgeräten & 35 & 55 \\
\hline Läuterung mit kleiner Motorsäge & 45 & 65 \\
\hline Läuterung mit Freischneider & 35 & 77 \\
\hline Wegebau & 23 & 56 \\
\hline Holzernte (Laubholz) & 44 & 41 \\
\hline Holzernte (Nadelholz) & 59 & 41 \\
\hline Sammelhieb & 59 & \\
\hline \hline
\end{tabular}

Aufgrund der dargestellten Unterteilung in Reine Arbeitszeit und Allgemeine Zeit werden die gemessenen Werte für die Haupttätigkeit der Forstwirte zum jeweiligen Messzeitpunkt lediglich mit dem entsprechenden Prozentsatz bei den Aktivitätsprotokollen eingerechnet. Die verbleibende Allgemeine Zeit wird zu den leichten Tätigkeiten im Stehen addiert.

Die Methode, mittels der Herzfrequenz einen akzeptablen Schätzwert für körperliche Aktivität zu errechnen, konnte nicht in die Praxis umgesetzt werden. Ob die Herzfrequenz eine adäquate Messgröße zur Berechnung des Energieumsatzes ist, wird in der Literatur kontrovers diskutiert (Payne et al. 1971; Spurr et al. 1988; Livingstone 1997; Schutz et al. 2001; Schutz 2003; Esliger 2005; Keytel et al. 2005; Fleischer 2009; Montgomery et a. 2009; Çalişkan \&

\footnotetext{
${ }^{12}$ Nach Kuratorium für Waldarbeit und Forsttechnik 2000 und REFA-Fachausschuss Forstwirtschaft 2004.
} 
Çağlar 2010; Erdogan et al. 2010; Spierer \& Hagins 2011). Trotz der praktikablen und kostengünstigen Handhabung scheiterte jedoch die Umsetzung bei der Messung während forstwirtlicher Tätigkeiten (gestörte Telemetrie, Unvereinbarkeit vom Tragen der Pulsuhr und der Ausführung der beruflichen Anforderungen). Darüber hinaus stellten Redding et al. (2004) fest, dass sich der Zusammenhang zwischen Herzfrequenz und Sauerstoffverbrauch auf Steady-state-Belastungen bezieht und somit bei niedrigen bis moderaten Intensitäten mit gelegentlichen anaeroben Spitzen keine Anwendung findet.

Mit dem erarbeiteten Auswertungsverfahren der Aktivitätsprotokolle werden für die Probanden individuelle Energieumsätze geschätzt und berechnet, ergänzt durch die aus der indirekten Kalorimetrie gemessenen Werte bei der Haupttätigkeit zum jeweiligen Messzeitpunkt. Somit kann zwischen dem Energieverbrauch der gesamten Woche, der einzelnen Tage und der Freizeit sowie der beruflichen Tätigkeit unterschieden werden.

\subsection{4 Äußere Bedingungen}

Der Energiebedarf des menschlichen Organismus unterliegt mehreren Faktoren (vgl. Kapitel 2). Neben der körperlichen Aktivität werden deshalb von den Probanden für die Arbeitszeit äußere Einflussfaktoren protokolliert. Für jeden Messzeitraum zeichnen die Forstwirte mit Hilfe eines Protokolls (Anhang 6) die entsprechenden Informationen auf.

Die wichtigsten Einflussfaktoren auf eine auszuführende Tätigkeit sind der Arbeitsplatz selbst, Witterungsverhältnisse und das angewendete Arbeitsverfahren (REFA-Fachausschuss Forstwirtschaft 1991; Kuratorium für Waldarbeit und Forsttechnik 1999). Häufig werden qualitative Beschreibungen durchgeführt, um die genannten Faktoren mit vertretbarem Aufwand messen zu können. Diese erschwert jedoch die Auswertung und Interpretation der Ergebnisse, weshalb in der vorliegenden Erhebung Stufenskalen verwendet werden, um die Einflussfaktoren zu quantifizieren (ebd.).

Um die klimatischen Bedingungen zu erfassen, werden die Temperatur als auch das Wetter erhoben. Die Einteilung des Wetters erfolgt in den Kategorien bewölkt, Regen, Schnee, Sonne und Wind. Neben der Erfassung klimatischer Faktoren werden die Geländeverhältnisse protokolliert, um die Arbeitsplatzcharakterisierung bezüglich der Hangneigung, des Bewuchses und der besonderen Bestandesmerkmale zu erheben. Die Kategorisierung der Hangneigung findet nach folgenden Ausprägungen statt: 
Tabelle 6: Kategorisierung der Hangneigung. ${ }^{13}$

\begin{tabular}{|l|c|c|c|c|}
\hline \hline Hangneigung in \% & 0 & bis 15 & von 16 bis 35 & ab 36 \\
\hline Kategorie & Keine & leichte & mittlere & starke \\
\hline \hline
\end{tabular}

Die Beurteilung des Geländes und des Bewuchses erfolgt über die Kategorien keine oder geringe Behinderung, mittlere Behinderung, starke Behinderung oder sehr starke Behinderung (Kuratorium für Waldarbeit und Forsttechnik 2013).

Mit Hilfe der Protokolle über die äußeren Bedingungen können veränderte Energieumsätze diskutiert werden. Sowohl die Geländeverhältnisse als auch klimatische Bedingungen können u. U. Einfluss auf den Energieumsatz haben.

\subsubsection{Indirekte Kalorimetrie}

Die Messung des Energieumsatzes bei den Haupttätigkeiten der Forstwirte zu den jeweiligen Messzeitpunkten erfolgt über die Bestimmung der Sauerstoffaufnahme mit Hilfe der Spiroergometrie mit dem Gerät MetaMax3B der Firma Cortex (MM3B). Die hierbei gemessene, vom Körper aufgenommene, Sauerstoffmenge ermöglicht eine Bestimmung des Energieumsatzes, da bei jeder biologischen Oxidation Sauerstoff verbraucht wird (Triebig et al. 2003). Mit Hilfe des energetischen Äquivalentes und des respiratorischen Quotienten wird der Energieumsatz bestimmt (Mellerowicz 1979; Höppe 1984; de Marées 2003).

Die Gütekriterien der Messung mit MM3B wurden bisher nur an kleinen Stichproben und unter Laborbedingungen gemessen. Dabei konnte eine hohe Reliabilität festgestellt werden (Prieur et al. 2003; Vogler et al. 2010; Macfarlane \& Wong 2011). Bei der Betrachtung der $\mathrm{VO}_{2}$-Werte arbeiteten verschiedene Studien heraus, dass eine nicht ausreichende Validität vorhanden ist. Die $\mathrm{VO}_{2}$-Werte werden in einigen Studien unterschätzt (Prieur et al. 2003; Brehm et al. 2004; Laurent et al. 2008) und in anderen Studien überschätzt (Perkins et al. 2004; Vogler et al. 2010; Macfarlane \& Wong 2011). Bei der Auswertung der erhobenen Werte sollte dieser Aspekt berücksichtigt werden. Weitere Untersuchungen zu den Gütekriterien des MM3B wären, wie bereits von Meyer (2005) konstatiert, wünschenswert.

Das Ergospirometrie-System MM3B zeichnet sich durch eine mobile, um die Schulter des Probanden positionierte Messeinheit aus (Zimmer 2010; vgl. Abbildung 10). Diese Methode ermöglicht daher die Messung am sich bewegenden Menschen, in der vorliegenden Untersuchung am Forstwirt, während der Ausführung der unterschiedlichen beruflichen Tätigkeiten.

\footnotetext{
${ }^{13}$ Kuratorium für Waldarbeit und Forsttechnik 2013.
} 


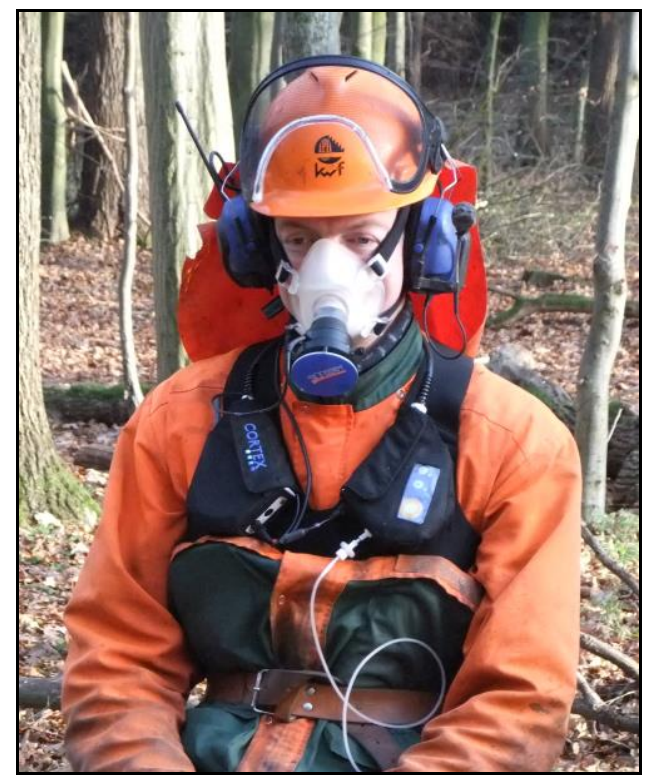

Abbildung 10: Forstwirt mit angelegtem Spiroergometrie-System und speziellem Gesichtsschutz.

Ein speziell angefertigter Gesichtsschutz mit Aussparung in dem Bereich, in dem sich die Atemmaske befindet, gewährleistet die notwendige Sicherheit auch bei Arbeiten, bei denen das Tragen eines Gesichtsschutzes obligatorisch ist.

Die indirekte Kalorimetrie im offenen System hat sich besonders bei Untersuchungen im Feld durchgesetzt. Der Proband atmet dabei über eine Gasuhr Frischluft ein, die das Inspirationsvolumen misst. Gasanalysegeräte entnehmen ständig die $\mathrm{O}_{2}$ - und $\mathrm{CO}_{2}$-Konzentrationen in der Exspirationsluft (Mellerowicz 1979; Golenhofen 2006; Persson 2007). Anhand des Sauerstoffverbrauchs $\left(\mathrm{VO}_{2}\right)$ und der Kohlenstoffdioxidabgabe $\left(\mathrm{VCO}_{2}\right)$ wird der aktuelle Energieumsatz bestimmt (Mellerowicz 1979; Montoye et al. 1996; McArdle et al. 2000; Wirth 2003; Kreymann 2006; Persson 2007; Kroidl et al. 2010), da alle Energie freisetzenden Reaktionen im menschlichen Organismus Sauerstoff benötigen (Mellerowicz 1979; McArdle et al. 2000). Die Sauerstoffaufnahme, gemessen in 1/ min, bezeichnet die Menge an Sauerstoff, die vom Organismus zur Energiebereitstellung innerhalb einer Minute im Gewebe umgesetzt wird. Dabei dient folgende Gleichung zur Berechnung:

$$
\mathrm{VO}_{2}=\text { VE* (FI-FE) } \mathrm{O}_{2} \text {. }
$$

VE bezeichnet das Atemminutenvolumen. FI und FE stehen für die inspiratorische bzw. die exspiratorische Sauerstoffkonzentration (de Marées 2003; Kroidl et al. 2010). Dabei geht die indirekte Kalorimetrie davon aus, dass "bei der Oxidation der Nährstoffe eine stöchiometrische Beziehung [...] zwischen dem Verbrauch von Sauerstoff, der Bildung von $\mathrm{CO}_{2}$, der Menge "verbrannter" Substanz und der dabei freiwerdenden Energie besteht" (de Marées 2003). 
Um bei der Messung Exaktheit und Validität sicher zu stellen, wird, wie in der Literatur beschrieben, regelmäßig eine Kalibrierung des MM3B durchgeführt (Mc Ardle et al. 2000). In den folgenden Kapiteln werden die Erhebung der Spiroergometrie bei beruflichen Tätigkeiten als auch die Fahrradspiroergometrie dargestellt, die als ergänzende Methode bei vier Probanden durchgeführt wurde.

\subsubsection{Spiroergometrie}

Wenn ein Proband bei der indirekten Kalorimetrie bzw. Spirometrie Arbeit leistet, spricht man von Spiroergometrie (Persson 2007). Ergometrien sind „Verfahren, die die Beobachtung und Analyse physiologischer Parameter während körperlicher Belastung zum Ziel haben“ (Platen 2001) und werden eingesetzt, um physische Leistungen exakt dosieren und jederzeit reproduzieren zu können (Hollmann \& Hettinger 2000). Um den respiratorischen Gasstoffwechsel und die Atmung kontinuierlich zu erfassen, werden Spirometrien durchgeführt. Kombiniert man diese beiden Methoden, so spricht man von Spiroergometrien, mittels derer sich „qualitativ und quantitativ die Reaktion von Herz, Kreislauf, Atmung und Stoffwechsel auf muskuläre Arbeit sowie die kardiopulmonale Leistungsfähigkeit beurteilen lassen“ (Hollmann \& Hettinger 2000). Sie sind die am meisten genutzte und am besten standardisierte objektivierende Diagnostik des kardiopulmonalen Systems und des Energiestoffwechsels (Laube 2009a).

Während der Spiroergometrie werden kardiozirkulatorische, ventilatorische und den Gasaustausch betreffende Parameter bestimmt (Platen 2001; Friedrich 2007; Kroidl et al. 2010; Röcker 2010). Dabei ist eine breath-by-breath-Analyse der Standard heutiger Untersuchungen, jedoch liegt nicht unbedingt eine Datenausgabe für jeden einzelnen Atemzug vor. Vielmehr handelt es sich bei den gemessenen Werten um Durchschnittswerte innerhalb vorgesehener Zeitintervalle (Röcker 2010). Da in der vorliegenden Studie keine Lungenfunktionsprüfung angestrebt wird, sondern sich die Fragestellung nach dem Energieumsatz bei alltäglichen Belastungen richtet, liegt der Fokus auf folgenden kardiopulmonalen Parametern: Sauerstoffverbrauch $\left(\mathrm{VO}_{2}\right)$, Kohlendioxidproduktion $\left(\mathrm{VCO}_{2}\right)$ und respiratorischer Quotient (RQ) (Kroidl et al. 2010), wobei $\mathrm{VO}_{2}$ den Energiebedarf widerspiegelt (Laube 2009a).

Die spiroergometrische Untersuchung wird im Feld bei typischen, von den Jahreszeiten abhängigen Tätigkeiten der Forstwirte durchgeführt. Im Winter (t1) befinden sich alle Forstwirte in der Holzernte. Die Tätigkeiten im Frühjahr (t2) verteilen sich auf Sammelhieb und unterschiedliche Pflanztechniken. Variierende Tätigkeiten sind im Sommer (t3) von den Forstwirten durchzuführen, die sich von der Holzernte über die Jungwuchspflege bis hin zum Wegebau erstrecken (vgl. Kapitel 5.2). 
Das offensystemische Spirometer lässt sich ortsunabhängig im Wald einsetzen und ermöglicht dank seiner Telemetrie und der Miniaturisierung einen mobilen Einsatz. Durch das geringe Gewicht und die körpernahe Fixierung kommt es lediglich zu einer minimalen Beeinträchtigung des Probanden (de Marées 2003). Die Studie verfolgt die Fragestellung nach dem Energieumsatz der Forstwirte bei ihrer täglichen Belastung. Aus diesem Grund werden die Forstwirte bei ihren beruflichen Tätigkeiten mit durchschnittlichen bzw. gewohnten Anforderungen gemessen und nicht bei maximaler Leistung. Somit werden keine maximalen Werte bestimmt (Kroidl et al. 2010) und die Forstwirte werden nicht über ihre gewohnte Tätigkeit hinaus belastet.

$\mathrm{Zu}$ Beginn einer jeden Untersuchung wird die mobile Messeinheit um die Schultern des Probanden fixiert. Während der Kalibrierung der Gassensoren anhand der Umgebungsluft nimmt der Proband bereits eine sitzende Position ein. Anschließend wird dem Probanden die Gesichtsmaske aufgesetzt, so dass in den ersten drei Minuten die Spiroergometrie in ruhender, sitzender Position durchgeführt wird. Diese Zeit dient der Gewöhnung der Probanden an die Messeinheit und an das Atmen mit der Gesichtsmaske. Die verwendete SpiroergometrieHalbmaske hat sich in einer Untersuchung als die bequemste herausgestellt, so dass lediglich die Wärmeentwicklung unter dem Maskenkörper als unangenehm vom Probanden empfunden wird (Vogl 2004).

Eine Messung des Grundumsatzes ist in diesem Fall nicht möglich, da weder eine Nahrungskarenz von zwölf Stunden noch eine vorherige körperliche Inaktivität vorliegen (vgl. Kapitel 3). Da die Messung während der Arbeitszeit stattfindet, haben die Forstwirte in den meisten Fällen bereits vorher gearbeitet und demnach auch Nahrung zu sich genommen. Aus diesem Grund wird der Grundumsatz mit der Formel nach Mifflin berechnet, um einen exakteren (aber dennoch geschätzten) Wert für den Grundumsatz zu erlangen. Dieser Grundumsatz entspricht 1 MET (vgl. Kapitel 3.1 und 6.3.3). Der Tabelle 4 können die Faktoren entnommen werden, mit denen die Energieumsätze der einzelnen Tätigkeiten des gesamten Tages berechnet werden.

Die Werte für den Energieumsatz bei der jeweiligen Tätigkeit werden nicht mittels MET berechnet, sondern anhand der erhobenen Werte in der Spiroergometrie bestimmt. Hierzu wird der Energieumsatz in kcal/ min gemittelt. Dabei werden sowohl die Werte, die im Sitzen gemessen werden, als auch die Werte der ersten drei Minuten bei körperlicher Tätigkeit von der Berechnung der Mittelwerte ausgeschlossen (Lehmann 1961c). Dieser gemittelte Wert gibt nun den individuellen Energieumsatz in kcal/ min für die jeweilige Tätigkeit an und wird bei der Auswertung des Aktivitätsprotokolls verwendet. 
Die Spiroergometrie wird bei den Forstwirten zu jedem Messzeitpunkt durchschnittlich 30 Minuten durchgeführt. Eine Standardisierung der Tätigkeiten ist nicht möglich, da die Arbeitsabläufe selbst bei identischem Verfahren durch äußere Einflüsse bzw. Erschwernisse variabel gestaltet werden. Von einer Bewegungsanalyse im Labor wurde aufgrund der nicht zu berücksichtigenden Varianzen und Einflüsse abgesehen, zumal eine realitätsnahe Gestaltung der Arbeitsabläufe im Labor nicht möglich ist.

Um die erhobenen Werte des Energieumsatzes einer Leistung zuordnen zu können, haben vier Forstwirte zusätzlich zu allen drei Messzeitpunkten eine Fahrradspiroergometrie durchgeführt, die im nachfolgenden Kapitel erläutert wird.

\subsubsection{Fahrradspiroergometrie}

Wie bereits beschrieben werden bei einer Spiroergometrie kontinuierlich Parameter ermittelt, während eine definierte mechanische Leistung erbracht wird (Friedrich 2007). Die erbrachte Leistung ist dabei sowohl abhängig von Parametern der Energiegewinnung in der Muskulatur als auch von der Leistungsfähigkeit der konvektiven Systeme (Kroidl et al. 2010). Bei der nicht standardisierten Messung im Feld wird lediglich die Arbeitsschwere über die indirekte Kalorimetrie gemessen, jedoch kann die erbrachte Leistung nicht in physikalischen Maßen ausgedrückt werden. Deshalb wird bei vier Probanden zusätzlich eine Fahrradspiroergometrie durchgeführt, um die Werte der Feldstudie mit den Werten der Fahrradspiroergometrie vergleichen zu können und sie einem Belastungsbereich in Watt zuordnen zu können (Åstrand 1987). Die Fahrradspiroergometrie erfüllt somit durch die präzise Dosierbarkeit und die exakte Reproduzierbarkeit die Anforderung einer Leistungsuntersuchung, u. a. durch die geringen Variationsmöglichkeiten bei der Ausführung (Lehmann 1961a; Hollmann \& Hettinger 2000). Die Probanden absolvieren bei der Fahrradspiroergometrie drei Belastungsstufen à zehn Minuten. Die Belastungsdauer von zehn Minuten lehnt sich an die gemessene Zeit im Feld an und ermöglicht eine Messung, bei der sich die kardiorespiratorischen Systeme auf der neuen Belastungsstufe einpendeln können. Eine der drei Belastungsstufen wird mit $100 \mathrm{~W}$ angesetzt, die anderen beiden Belastungsstufen werden entsprechend der im Feld gemessenen Werte gewählt. Da es sich um eine lineare Beziehung zwischen der Arbeitsintensität (in Watt) und der Sauerstoffaufnahmegröße handelt, ist unter einmaliger Berücksichtigung der Sauerstoffaufnahme in Ruhe ein Abschätzen dieser Werte möglich (Hollmann \& Hettinger 2000; Scharhag-Rosenberger \& Schommer 2013).

Während der Vorstartsituation wird der Proband drei Minuten lang sitzend auf dem Fahrradergometer gemessen. Die Sitzhöhe ist zu allen drei Messzeitpunkten identisch, um die Belas- 
tung konstant zu halten (Kroidl 2010). Anschließend absolviert jeder Proband die für ihn geschätzten Belastungsstufen.

Bei der Auswertung werden die Energieumsätze der Probanden gemittelt. Dabei werden sowohl die ersten beiden Minuten als auch die letzte Minute jeder Belastungsstufe von der Berechnung ausgeschlossen, um die Phase der Anpassung an die neue Belastungsstufe und die Phase der Vorbereitung auf die nächste Belastungsstufe nicht einzurechnen. Die Probanden werden dazu angehalten, mit einer einheitlichen Drehzahl von 50 bis 60 Umdrehungen pro Minute zu fahren, um einen optimalen Wirkungsgrad zu erzielen (Hollmann \& Hettinger 2000; Rost \& Hollmann 1982). Die erhobenen Werte können nun mit den Werten der spiroergometrischen Messung im Feld verglichen werden und machen eine Zuordnung in Leistungsbereiche in Watt möglich.

\subsubsection{Operationalisierung der Variablen}

Die folgende Tabelle listet die Operationalisierung der hypothesenrelevanten Variablen auf und gibt einen Überblick über die dazugehörigen Merkmale und die Ausprägung bzw. Skalierung der Variablen. Sofern nicht anders vermerkt, werden fehlende Werte im Datensatz mit -99 von weiteren Berechnungen ausgeschlossen.

Tabelle 7: Operationalisierung der Variablen.

\begin{tabular}{|c|c|c|}
\hline Inhalt & Variablenname im Datensatz & Ausprägung \\
\hline Geburtsjahr des Probanden & Geburtsjahr & Wert entspricht Jahreszahl \\
\hline Körpergröße des Probanden & Größe & Wert entspricht Größße in cm \\
\hline Gewicht des Probanden in $\mathrm{t} 1$ & Gewicht_t1 & Wert entspricht Gewicht in $\mathrm{kg}$ \\
\hline Gewicht des Probanden in $\mathrm{t} 2$ & Gewicht_t2 & Wert entspricht Gewicht in kg \\
\hline Gewicht des Probanden in $\mathrm{t} 3$ & Gewicht_t3 & Wert entspricht Gewicht in $\mathrm{kg}$ \\
\hline BMI des Probanden in $\mathrm{t} 1$ & BMI_t1 & $\begin{array}{l}\text { Wert entspricht dem BMI (dimensions- } \\
\text { los) }\end{array}$ \\
\hline Sind Sie Raucher? & Raucher & $0=$ Nichtraucher, $1=$ Raucher \\
\hline Leiden Sie an Erkrankungen? & Erkrankungen & $0=$ ja; $1=$ nein \\
\hline Nehmen Sie Medikamente ein? & Medikamente & $0=$ ja; $1=$ nein \\
\hline Seit wann sind Sie im Beruf? & Berufsjahre & Wert entspricht Jahreszahl \\
\hline $\begin{array}{l}\text { Was ist Ihr höchster Bildungsab- } \\
\text { schluss? }\end{array}$ & Bildungsabschluss & $\begin{array}{l}0=\text { kein Schulabschluss, } \\
1=\text { Hauptschulabschluss, } 2=\text { mittlere } \\
\text { Reife, } 3=\text { =Fachhochschulreife, } 4=\text { Abitur, } \\
5=\text { Fachhochschulabschluss, } \\
6=\text { sonstiges }\end{array}$ \\
\hline $\begin{array}{l}\text { Mittelwert der Energiebilanz in } \\
\text { einer Woche aller Probanden aller } \\
\text { Messzeitpunkte }\end{array}$ & Bilanz_MW_Woche & Wert entspricht Bilanz in kcal \\
\hline Energiebilanz aller Probanden in $\mathrm{t} 1$ & Bilanz_t1_gesamt & Wert entspricht Bilanz in kcal \\
\hline $\begin{array}{l}\text { Mittelwert der Energiebilanz pro } \\
\text { Tag in } \mathrm{t} 1\end{array}$ & Bilanz_t1_MW_d & Wert entspricht Bilanz in kcal \\
\hline $\begin{array}{l}\text { Energiebilanz an Tag } 1 \text { (Montag) } \\
\text { in t1 }\end{array}$ & Bilanz_t1_d1 & Wert entspricht Bilanz in kcal \\
\hline $\begin{array}{l}\text { Energiebilanz an Tag } 2 \text { (Dienstag) } \\
\text { in } \mathrm{t} 1\end{array}$ & Bilanz_t1_d2 & Wert entspricht Bilanz in kcal \\
\hline
\end{tabular}




\begin{tabular}{|c|c|c|}
\hline $\begin{array}{l}\text { Energiebilanz an Tag } 3 \text { (Mittwoch) } \\
\text { in } \mathrm{t} 1\end{array}$ & Bilanz_t1_d3 & Wert entspricht Bilanz in kcal \\
\hline $\begin{array}{l}\text { Energiebilanz an Tag } 4 \text { (Donners- } \\
\text { tag) in t1 }\end{array}$ & Bilanz_t1_d4 & Wert entspricht Bilanz in kcal \\
\hline $\begin{array}{l}\text { Energiebilanz an Tag } 5 \text { (Freitag) } \\
\text { in } \mathrm{t} 1\end{array}$ & Bilanz_t1_d5 & Wert entspricht Bilanz in kcal \\
\hline $\begin{array}{l}\text { Energiebilanz an Tag } 6 \text { (Samstag) } \\
\text { in } \mathrm{t} 1\end{array}$ & Bilanz_t1_d6 & Wert entspricht Bilanz in kcal \\
\hline $\begin{array}{l}\text { Energiebilanz an Tag } 7 \text { (Sonntag) } \\
\text { in } \mathrm{t} 1\end{array}$ & Bilanz_t1_d7 & Wert entspricht Bilanz in kcal \\
\hline $\begin{array}{l}\text { Mittelwert der Energiebilanzen an } \\
\text { Werktagen (Tag } 1 \text { bis Tag 4) in t1 }\end{array}$ & Bilanz_t1_wt_modo & Wert entspricht Bilanz in kcal \\
\hline $\begin{array}{l}\text { Mittelwert der Energiebilanzen am } \\
\text { Wochenende (Tag } 6 \text { und Tag 7) in } \\
\text { t1 }\end{array}$ & Bilanz_t1_we & Wert entspricht Bilanz in kcal \\
\hline $\begin{array}{l}\text { Mittelwert des Grundumsatzes } \\
\text { nach der Mifflin-Formel ( } 11-\mathrm{t} 3)\end{array}$ & Kcal_RMR_Miff_MW & Wert entspricht Grundumsatz in kcal \\
\hline $\begin{array}{l}\text { Grundumsatz nach der Mifflin- } \\
\text { Formel in } \mathrm{t} 1\end{array}$ & Kcal_RMR_Miff_t1 & Wert entspricht Grundumsatz in kcal \\
\hline $\begin{array}{l}\text { Mittelwert des spiroergometrisch } \\
\text { gemessenen Ruheumsatzes ( } t 1-\mathrm{t} 3 \text { ) }\end{array}$ & Kcal_RMR_Spiro_MW & Wert entspricht Grundumsatz in kcal \\
\hline $\begin{array}{l}\text { Mittelwert des spiroergometrisch } \\
\text { gemessenen Energieumsatzes bei } \\
\text { Arbeit }(\mathrm{t} 1-\mathrm{t} 3)\end{array}$ & Kcal_arb_Spiro_MW & Wert entspricht Arbeitsumsatz in kcal \\
\hline Hautfaltendicke in $\mathrm{t} 2$ & Caliper_t2_mm & Wert entspricht Hautfaltendicke in $\mathrm{mm}$ \\
\hline Körperfettanteil in t2 & Caliper_t2_Prozent & Wert entspricht Körperfettanteil in \% \\
\hline $\begin{array}{l}\text { Wetter am Vormittag von Tag } 1 \\
\text { (Montag) in } \mathrm{t} 1\end{array}$ & Wetter_t1_d1_vm & $\begin{array}{l}0=\text { trocken, } 1=\text { Regen, } 2=\text { Schnee, } \\
3=\text { Sonne, } 4=\text { Wind }\end{array}$ \\
\hline $\begin{array}{l}\text { Wetter am Nachmittag von Tag } 2 \\
\text { (Dienstag) in } \mathrm{t} 1\end{array}$ & Wetter_t1_d2_nm & $\begin{array}{l}0=\text { trocken, } 1=\text { Regen, } 2=\text { Schnee, } \\
3=\text { Sonne, } 4=\text { Wind }\end{array}$ \\
\hline $\begin{array}{l}\text { Temperatur am Vormittag von Tag } \\
1 \text { in } 11\end{array}$ & Temp_t1_d1_vm & Wert entspricht der Temperatur in ${ }^{\circ} \mathrm{C}$ \\
\hline Hangneigung an Tag 1 in $\mathrm{t} 1$ & Hangneigung_t1_d1 & $\begin{array}{l}0=\text { keine }, 1=\text { leichte, } 2=\text { mittlere } \\
3=\text { starke Hangneigung }\end{array}$ \\
\hline $\begin{array}{l}\text { Bodenbewuchs und Behinderung } \\
\text { an Tag } 1 \text { in } \mathrm{t} 1\end{array}$ & Bewuchs_t1_d1 & $\begin{array}{l}0=\text { keine oder geringe, } 1=\text { mittlere, } \\
2=\text { starke, } 3=\text { sehr starke Behinderung }\end{array}$ \\
\hline Energieumsatz der Woche in $\mathrm{t} 1$ & Akt_t1_gesamt & $\begin{array}{l}\text { Wert entspricht dem Energieumsatz in } \\
\text { kcal }\end{array}$ \\
\hline $\begin{array}{l}\text { Energieumsatz der Woche im } \\
\text { Schlaf in } \mathrm{t} 1\end{array}$ & Akt_t1_schlaf_gesamt & $\begin{array}{l}\text { Wert entspricht dem Energieumsatz in } \\
\text { kcal }\end{array}$ \\
\hline $\begin{array}{l}\text { Energieumsatz der Woche im Be- } \\
\text { ruf in } \mathrm{t} 1\end{array}$ & Akt_t1_beruf_gesamt & $\begin{array}{l}\text { Wert entspricht dem Energieumsatz in } \\
\text { kcal }\end{array}$ \\
\hline $\begin{array}{l}\text { Energieumsatz der Woche in der } \\
\text { Freizeit in } \mathrm{t} 1\end{array}$ & Akt_t1_frei_gesamt & $\begin{array}{l}\text { Wert entspricht dem Energieumsatz in } \\
\text { kcal }\end{array}$ \\
\hline $\begin{array}{l}\text { Energieumsatz der Woche beim } \\
\text { Sport in } \mathrm{t} 1\end{array}$ & Akt_t1_sport_gesamt & $\begin{array}{l}\text { Wert entspricht dem Energieumsatz in } \\
\text { kcal }\end{array}$ \\
\hline Energieumsatz an Werktagen in $\mathrm{t} 1$ & Akt_t1_wt_modo & $\begin{array}{l}\text { Wert entspricht dem Energieumsatz in } \\
\text { kcal }\end{array}$ \\
\hline $\begin{array}{l}\text { Energieumsatz am Wochenende in } \\
\mathrm{t} 1\end{array}$ & Akt_t1_we & $\begin{array}{l}\text { Wert entspricht dem Energieumsatz in } \\
\text { kcal }\end{array}$ \\
\hline Energieaufnahme der Woche in $\mathrm{t} 1$ & Ern_t1_kcal_gesamt & $\begin{array}{l}\text { Wert entspricht der Energieaufnahme } \\
\text { in kcal }\end{array}$ \\
\hline Fettanteil der Ernährung in $\mathrm{t} 1$ & Ern_t1_fett_Prozent & $\begin{array}{l}\text { Wert entspricht dem Fettanteil der } \\
\text { Ernährung in \% }\end{array}$ \\
\hline $\begin{array}{l}\text { Kohlenhydratanteil der Ernährung } \\
\text { in t1 }\end{array}$ & Ern_t1_kh_Prozent & $\begin{array}{l}\text { Wert entspricht dem Kohlenhydratan- } \\
\text { teil der Ernährung in \% }\end{array}$ \\
\hline Eiweißanteil der Ernährung in t1 & Ern_t1_eiw_Prozent & $\begin{array}{l}\text { Wert entspricht dem Eiweißanteil der } \\
\text { Ernährung in \% }\end{array}$ \\
\hline Relative Eiweißzufuhr in t1 & Ern_t1_eiw_rel_MW & $\begin{array}{l}\text { Wert entspricht der relativen Eiweißzu- } \\
\text { fuhr in } \mathrm{g} / \mathrm{kg} \mathrm{KG}\end{array}$ \\
\hline
\end{tabular}




\begin{tabular}{|c|c|c|}
\hline $\begin{array}{l}\text { Mittelwert der Flüssigkeitszufuhr } \\
\text { pro Tag in t1 }\end{array}$ & Ern_t1_was_MW & $\begin{array}{l}\text { Wert entspricht der Flüssigkeitszufuhr } \\
\text { in } l\end{array}$ \\
\hline $\begin{array}{l}\text { Mittelwert des Alkoholkonsums } \\
\text { pro Tag in } \mathrm{t} 1\end{array}$ & Ern_t1_alk_MW & $\begin{array}{l}\text { Wert entspricht dem Alkoholkonsum in } \\
g\end{array}$ \\
\hline $\begin{array}{l}\text { Fettanteil der Ernährung an Tag } 1 \\
\text { in t1 }\end{array}$ & Ern_t1_d1_fett_Prozent & $\begin{array}{l}\text { Wert entspricht dem Fettanteil der } \\
\text { Ernährung in } \%\end{array}$ \\
\hline Sauerstoffaufnahme in Ruhe in t1 & Spi_t1_O2_ruhe & $\begin{array}{l}\text { Wert entspricht der Sauerstoffaufnah- } \\
\text { me in Ruhe in l/min }\end{array}$ \\
\hline $\begin{array}{l}\text { Sauerstoffaufnahme bei Belastung } \\
\text { in t1 }\end{array}$ & Spi_t1_O2_bel & $\begin{array}{l}\text { Wert entspricht der Sauerstoffaufnah- } \\
\text { me in Ruhe in } 1 / \text { min }\end{array}$ \\
\hline RQ in $\mathrm{t} 1$ & Spi_t1_RQ & $\begin{array}{l}\text { Wert entspricht dem } R Q \text { (dimensions- } \\
\text { los) }\end{array}$ \\
\hline Absoluter Energieumsatz in t1 & Spi_t1_EU_abs & $\begin{array}{l}\text { Wert entspricht dem Energieumsatz in } \\
\mathrm{kcal} / \mathrm{min}\end{array}$ \\
\hline Relativer Energieumsatz in $\mathrm{t} 1$ & Spi_t1_EU_rel & $\begin{array}{l}\text { Wert entspricht dem Energieumsatz in } \\
\mathrm{kcal} / \mathrm{min} / \mathrm{kg} \mathrm{KG}\end{array}$ \\
\hline $\begin{array}{l}\text { Temeratur bei der spiroergometri- } \\
\text { schen Messung in } \mathrm{t} 1\end{array}$ & Spi_t1_temp & Wert entspricht der Temperatur in ${ }^{\circ} \mathrm{C}$ \\
\hline $\begin{array}{l}\text { Hangneigung bei der spiroergomet- } \\
\text { rischen Messung in } \mathrm{t} 1\end{array}$ & Spi_t1_Hangneigung & $\begin{array}{l}0=\text { keine, } 1=\text { leichte, } 2=\text { mittlere } \\
3=\text { starke Hangneigung }\end{array}$ \\
\hline $\begin{array}{l}\text { Bodenbewuchs und Behinderung } \\
\text { bei der spiroergometrischen Mes- } \\
\text { sung in } \mathrm{t} 1\end{array}$ & Spi_t1_Bewuchs & $\begin{array}{l}0=\text { keine oder geringe, } 1=\text { mittlere, } \\
2=\text { starke, } 3=\text { sehr starke Behinderung }\end{array}$ \\
\hline $\begin{array}{l}\text { Wattzahl } 1 \text { bei Fahrradspiroergo- } \\
\text { metrie in } \mathrm{t} 1\end{array}$ & FE_t1_W1 & Wert entspricht der Leistung in $W$ \\
\hline $\begin{array}{l}\text { Wattzahl } 2 \text { bei Fahrradspiroergo- } \\
\text { metrie in } \mathrm{t} 1\end{array}$ & FE_t1_W2 & Wert entspricht der Leistung in $W$ \\
\hline $\begin{array}{l}\text { Wattzahl } 3 \text { bei Fahrradspiroergo- } \\
\text { metrie in } \mathrm{t} 1\end{array}$ & FE_t1_W3 & Wert entspricht der Leistung in $W$ \\
\hline $\begin{array}{l}\text { Sauerstoffaufnahme bei Wattzahl } 1 \\
\text { bei Fahrradspiroergometrie in t1 }\end{array}$ & FE_t1_O2_W1 & $\begin{array}{l}\text { Wert entspricht der Sauerstoffaufnah- } \\
\text { me in } l / \text { min }\end{array}$ \\
\hline $\begin{array}{l}\text { Absoluter Energieumsatz bei Watt- } \\
\text { zahl } 1 \text { bei Fahrradspiroergometrie } \\
\text { in } \mathrm{t} 1\end{array}$ & FE_t1_EU_abs_W1 & $\begin{array}{l}\text { Wert entspricht dem Energieumsatz in } \\
\mathrm{kcal} / \mathrm{min}\end{array}$ \\
\hline $\begin{array}{l}\text { Relativer Energieumsatz bei Watt- } \\
\text { zahl } 1 \text { bei Fahrradspiroergometrie } \\
\text { in t1 }\end{array}$ & FE_t1_EU_rel_W1 & $\begin{array}{l}\text { Wert entspricht dem Energieumsatz in } \\
\mathrm{kcal} / \mathrm{min} / \mathrm{kg} \mathrm{KG}\end{array}$ \\
\hline $\begin{array}{l}\text { RQ bei Wattzahl } 1 \text { bei Fahrradspi- } \\
\text { roergometrie }\end{array}$ & FE_t1_RQ_W1 & $\begin{array}{l}\text { Wert entspricht dem } R Q \text { (dimensions- } \\
\text { los) }\end{array}$ \\
\hline $\begin{array}{l}\text { Mittelwert des Fettanteils der Er- } \\
\text { nährung (t1-t3) }\end{array}$ & ern_fett_gesamt & Wert entspricht dem Fettanteil in \% \\
\hline $\begin{array}{l}\text { Mittelwert des Kohlenhydratanteils } \\
\text { der Ernährung (t1-t3) }\end{array}$ & ern_kh_gesamt & $\begin{array}{l}\text { Wert entspricht dem Kohlenhydratan- } \\
\text { teil in } \%\end{array}$ \\
\hline $\begin{array}{l}\text { Mittelwert des Eiweißanteils der } \\
\text { Ernährung (t1-t3) }\end{array}$ & ern_eiw_gesamt & Wert entspricht dem Eiweißanteil in \% \\
\hline
\end{tabular}

\subsubsection{Statistische Auswertung der Ergebnisse}

Die statistische Auswertung des Datenmaterials erfolgt mit Hilfe des Statistikprogramms IBM SPSS Statistics sowie mit Microsoft Excel in der jeweils aktuellen Version. Die Analyse der Ernährungsprotokolle findet zusätzlich unter Verwendung der Software DGE-PC statt. Als Analyseverfahren wird die deskriptive Statistik angewendet. Bei der Auswertung in der vorliegenden Arbeit werden überwiegend folgende statistischen Parameter verwendet: Mittelwert (MW), Standardabweichung (SD), Minimum (Min), Maximum (Max) und Signifikanzwert. 


\section{$7 \quad$ Ergebnisdarstellung}

In den folgenden Kapiteln werden die Ergebnisse der in Kapitel 6.3 vorgestellten Erhebungsinstrumente dargestellt. Die Prüfung der Hypothesen findet integrativ statt. Die Anzahl der Probanden für die Studie beträgt $\mathrm{n}=14$. Alle Probanden sind zu den drei Messzeitpunkten der Protokollierung nachgekommen und haben an der spiroergometrischen Untersuchung am Arbeitsplatz teilgenommen. An der Fahrradergometeruntersuchung nahmen FW01, FW03, FW04 und FW05 zu allen drei Messzeitpunkten teil.

Nachdem zunächst das Probandenkollektiv beschrieben wird, werden in den nachfolgenden Kapiteln die Ergebnisse der Ernährungsprotokolle und der Aktivitätsprotokolle dargestellt. Die Ergebnisse der indirekten Kalorimetrie folgen auf die Darstellung der äußeren Bedingungen und die Werte der Kalipermetrie. Abschließend werden die Energiebilanz und Korrelationen zwischen den einzelnen Parametern betrachtet.

\subsection{Anthropometrische und soziodemografische Daten}

An der Studie nahmen 14 Forstwirte teil. Tabelle 8 stellt die anthropometrischen Daten der Stichprobe dar. Das Durchschnittsalter der Probanden beträgt im Messzeitraum 40 Jahre. Der jüngste Proband ist FW02 mit 21 Jahren, die beiden ältesten Probanden sind FW05 und FW13 mit einem Alter von 51 Jahren. Die Probanden weisen eine durchschnittliche Körpergröße von $180 \mathrm{~cm}$ auf. Das durchschnittliche Körpergewicht steigt von t1 zu t3 leicht an mit Werten von $\mathrm{t} 1=87 \mathrm{~kg}, \mathrm{t} 2=88 \mathrm{~kg}$ und $\mathrm{t} 3=89 \mathrm{~kg}$. Das ansteigende Körpergewicht beeinflusst den BMI, der von 26,9 (t1) auf 27,3 (t3) ansteigt. Diese Werte liegen im übergewichtigen Bereich, der die Spanne zwischen 25 und 30 beschreibt (Roche Lexikon Medizin 1998; Kasper 2000; WHO 2000; de Marées 2003; Wirth 2003; Stensel 2008; Thiel 2011). 
Tabelle 8: Häufigkeitstabelle zu den Items Alter, Größe, Gewicht und BMI.

\begin{tabular}{|l|r|r|r|r|r|r|r|r|}
\hline \hline & Geburtsjahr & $\begin{array}{c}\text { Größe } \\
\text { (in cm) }\end{array}$ & $\begin{array}{c}\text { Gewicht } \\
\text { (in kg) } \\
\text { in t1 }\end{array}$ & $\begin{array}{c}\text { Gewicht } \\
\text { (in kg) } \\
\text { in t2 }\end{array}$ & $\begin{array}{c}\text { Gewicht } \\
\text { (in kg) } \\
\text { in t3 }\end{array}$ & $\begin{array}{c}\text { BMI in } \\
\text { t1 }\end{array}$ & $\begin{array}{c}\text { BMI in } \\
\text { t2 }\end{array}$ & $\begin{array}{c}\text { BMI in } \\
\text { t3 }\end{array}$ \\
\hline $\mathrm{N}$ & 14 & 14 & 14 & 14 & 14 & 14 & 14 & 14 \\
\hline Mittelwert & 1972 & 180,5 & 87,0 & 88,0 & 89,5 & 26,90 & 26,94 & 27,33 \\
\hline Median & 1969 & 181,5 & 83,5 & 85,5 & 84,2 & 26,4 & 26,8 & 26,4 \\
\hline Standardabweichung & 9,4 & 9,51 & 13,17 & 15,0 & 16,4 & 3,1 & 3,5 & 3,7 \\
\hline \hline
\end{tabular}

Der Körperfettanteil konnte nur in $\mathrm{t} 2$ und $\mathrm{t} 3$ festgestellt werden, da in $\mathrm{t} 1$ der Kaliper nicht zur Verfügung stand. Die Ergebnisse werden in Tabelle 9 dargestellt. Der Körperfettanteil beträgt im Durchschnitt 19,36\% in t2 und 20,81\% in t3. Die Werte streuen im Sommer geringfügig höher. Der minimale Wert $\left(\mathrm{t} 2_{\min }=9,5 ; \mathrm{t} 3_{\min }=10,9\right)$, sowie der maximale Wert $\left(\mathrm{t} 3_{\max }=24,6\right.$; $\mathrm{t} 3_{\max }=27,8$ ) haben sich leicht erhöht. Beide Werte befinden sich somit im Übergang vom normalgewichtigen zum adipösen Bereich, der die Werte 20-25\% umfasst (de Marées 2003).

Tabelle 9: Körperfettanteil in \% in $\mathbf{t} 2$ und $\mathrm{t} 3$.

\begin{tabular}{|l|r|r|}
\hline \hline & Körperfettanteil in t2 & Körperfettanteil in t3 \\
\hline $\mathrm{N}$ & 14 & 14 \\
\hline Minimum & 9,5 & 10,9 \\
\hline Maximum & 24,6 & 27,8 \\
\hline Mittelwert & 19,36 & 20,81 \\
\hline \hline
\end{tabular}

Vier der Probanden sind Raucher (FW07, FW10, FW13, FW14). Zwei Probanden (FW04, FW06) leiden unter Bluthochdruck, wobei nur FW06 ein blutdrucksenkendes Medikament einnimmt.

Bei der Frage nach dem höchsten Bildungsabschluss gaben jeweils sieben Forstwirte den Hauptschulabschluss bzw. die mittlere Reife an. Alle für die Niedersächsischen Landesforsten tätigen Forstwirte haben eine abgeschlossene Ausbildung zum Forstwirt.

\subsection{Ernährungsprotokoll}

Die Ernährungsprotokolle wurden mit der Software DGE-PC ausgewertet. Mittels des Programms lassen sich verschiedene Variablen ermitteln. Für die Fragestellung der vorliegenden Studie wird im Folgenden zunächst die Energieaufnahme (in kcal) der Forstwirte dargestellt. Anschließend wird die prozentuale Zusammensetzung der Ernährung aufgezeigt.

Tabelle 10 gibt einen Überblick über die aufgenommene Energie zu den drei Messzeitpunkten. Neben der Energieaufnahme in der gesamten Woche werden auch die Mittelwerte für 
einen einzelnen Tag angegeben. Hierbei ist eine abfallende Tendenz von t $1 \mathrm{zu}$ t 3 zu erkennen mit Mittelwerten einer Energieaufnahme pro Woche von $21948 \mathrm{kcal}$ in t1; $20801 \mathrm{kcal}$ in t2 und $20017 \mathrm{kcal}$ in t3. Auffällig sind die hohen Standardabweichungen, die der geringen Probandenzahl zu schulden sind, die jedoch von t1 zu t3 leicht abnehmen.

Hypothese 3 sagt aus, dass die Energieaufnahme im Winter kalorienreicher ist als im Frühjahr und im Sommer. Auffällig ist an den Ergebnissen zunächst, dass die durchschnittlichen Nahrungsaufnahmewerte in kcal nur verhältnismäßig geringfügig zwischen den Jahreszeiten schwanken (21948,50 kcal im Winter gegenüber 20017 kcal im Sommer). Tendenzielle Unterschiede lassen sich jedoch aus der Ernährung am Wochenende im Winter gegenüber dem Frühling ableiten $(\mathrm{p}=0,076(0,152 / 2)=0,0057)$ und im Winter gegenüber dem Sommer $(\mathrm{p}=$ 0,0935).

Um eine differenzierte Betrachtung der Energieaufnahme an Werktagen sowie am Wochenende zu ermöglichen, wird zusätzlich die Energieaufnahme an Werktagen (von Montag bis Donnerstag) als auch am Wochenende (von Samstag bis Sonntag) angegeben. Aufgrund der kurzen Arbeitszeit und der von den Werktagen abweichenden Freizeitaktivität am Freitag, fließen die Werte der Freitage nicht mit in die Auswertung ein.

Sowohl bei den Mittelwerten der Energieaufnahme an Werktagen als auch am Wochenende ist eine abfallende Tendenz von $\mathrm{t} 1 \mathrm{zu}$ t3 zu erkennen. Bei einem Vergleich der Energieaufnahme zwischen Werktagen und Wochenende ist zu allen drei Messzeitpunkten eine erhöhte Energieaufnahme am Wochenende zu verzeichnen. In $\mathrm{t} 1$ beträgt die Differenz der durchschnittlichen täglichen Energieaufnahme $518 \mathrm{kcal}$. In t2 beträgt die Differenz $153 \mathrm{kcal}$ und in t3 88 kcal.

Darüber hinaus ist zu erkennen, dass die Energieaufnahme am Wochenende (Samstag und Sonntag) den deutlichsten Unterschied zwischen den einzelnen Messzeitpunkten aufweist. In t1 beträgt der Mittelwert $3555 \mathrm{kcal}$, in t2 $3060 \mathrm{kcal}$ und in t3 $2908 \mathrm{kcal}$, so dass eine Diskrepanz von bis zu $650 \mathrm{kcal}$ pro Tag vorliegt. 
Tabelle 10: Energieaufnahme in kcal in $\mathrm{t} 1, \mathrm{t} 2$ und $\mathrm{t} 3$.

\begin{tabular}{|c|c|c|c|c|c|}
\hline & $\mathrm{N}$ & Minimum & Maximum & Mittelwert & Standardabweichung \\
\hline $\begin{array}{l}\text { Energieaufnahme in } \mathrm{t} 1 \text { pro } \\
\text { Woche }\end{array}$ & 14 & 13345 & 32404 & 21948 & 5286 \\
\hline $\begin{array}{l}\text { Durchschnittliche Energie- } \\
\text { aufnahme in t1 pro Tag }\end{array}$ & 14 & 1906 & 4629 & 3135 & 755 \\
\hline $\begin{array}{l}\text { Energieaufnahme in } \mathrm{t} 2 \text { pro } \\
\text { Woche }\end{array}$ & 14 & 13982 & 28068 & 20801 & 3888 \\
\hline $\begin{array}{l}\text { Durchschnittliche Energie- } \\
\text { aufnahme in } \mathrm{t} 2 \text { pro Tag }\end{array}$ & 14 & 1997 & 4010 & 2971 & 555 \\
\hline $\begin{array}{l}\text { Energieaufnahme in } \mathrm{t} 3 \text { pro } \\
\text { Woche }\end{array}$ & 14 & 16358 & 27864 & 20017 & 3033 \\
\hline $\begin{array}{l}\text { Durchschnittliche Energie- } \\
\text { aufnahme in } \mathrm{t} 3 \text { pro Tag }\end{array}$ & 14 & 2337 & 3981 & 2859 & 433 \\
\hline $\begin{array}{l}\text { durchschnittliche Energieauf- } \\
\text { nahme in } \mathrm{t} 1 \text { an Werktagen }\end{array}$ & 14 & 1781 & 4470 & 3037 & 662 \\
\hline $\begin{array}{l}\text { durchschnittliche Energieauf- } \\
\text { nahme in } \mathrm{t} 2 \text { an Werktagen }\end{array}$ & 14 & 1914 & 3564 & 2907 & 486 \\
\hline $\begin{array}{l}\text { durchschnittliche Energieauf- } \\
\text { nahme in } \mathrm{t} 3 \text { an Werktagen }\end{array}$ & 14 & 2006 & 3492 & 2820 & 462 \\
\hline $\begin{array}{l}\text { durchschnittliche Energieauf- } \\
\text { nahme in t1 am Wochenende }\end{array}$ & 14 & 2102 & 5292 & 3555 & 1114 \\
\hline $\begin{array}{l}\text { durchschnittliche Energieauf- } \\
\text { nahme in } \mathrm{t} 2 \text { am Wochenende }\end{array}$ & 14 & 1611 & 4780 & 3060 & 1009 \\
\hline $\begin{array}{l}\text { durchschnittliche Energieauf- } \\
\text { nahme in } \mathrm{t} 3 \text { am Wochenende }\end{array}$ & 14 & 990 & 5725 & 2908 & 1289 \\
\hline
\end{tabular}

Beim Test der gepaarten Variablen kann zwischen der Energieaufnahme an Wochentagen und der Energieaufnahme am Wochenende zu t1 eine signifikante Veränderung verzeichnet werden. Außerdem kann eine leicht signifikante Veränderung der Energieaufnahme von t1 zu t2 und $\mathrm{t} 3$ festgestellt werden.

Hypothese 2, dass sich das Ernährungsverhalten der Forstwirte signifikant zwischen Werktagen und dem Wochenende unterscheidet, kann somit partial verifiziert werden. Im Vergleich der Energieaufnahmen an Werktagen (Montag bis Donnerstag) zu Wochenendtagen zeigt sich eine absolute Differenz von $518 \mathrm{kcal} / \mathrm{d}$ in $\mathrm{t} 1,153 \mathrm{kcal} / \mathrm{d}$ in $\mathrm{t} 2$ und $88 \mathrm{kcal} / \mathrm{d}$ in t3.

Dieser Unterschied lässt sich auch statistisch belegen. Im Winter unterscheidet sich die Energieaufnahme an Werktagen signifikant von der Energieaufnahme am Wochenende $(\mathrm{p}=0,025)$, wobei eindeutig am Wochenende mehr konsumiert wird. Somit kann die Hypothese für den Messzeitpunkt im Winter verifiziert werden. 
Tabelle 11: Vergleich der durchschnittlichen Energieaufnahmen zu verschiedenen Zeitpunkten. Test bei gepaarten Stichproben

\begin{tabular}{|c|c|c|c|c|c|c|c|c|c|}
\hline & & \multicolumn{5}{|c|}{ Gepaarte Differenzen } & \multirow[b]{3}{*}{$\mathrm{T}$} & \multirow[b]{3}{*}{ df } & \multirow{3}{*}{$\begin{array}{l}\text { Sig. } \\
(2- \\
\text { seitig) } \\
\end{array}$} \\
\hline & & \multirow[b]{2}{*}{$\begin{array}{l}\text { Mittel- } \\
\text { wert }\end{array}$} & \multirow[b]{2}{*}{$\begin{array}{c}\text { Standard- } \\
\text { abweichung }\end{array}$} & \multirow{2}{*}{$\begin{array}{c}\text { Standardfehler } \\
\text { des Mittelwer- } \\
\text { tes }\end{array}$} & \multicolumn{2}{|c|}{$\begin{array}{c}\text { 95\% Konfiden- } \\
\text { zintervall der } \\
\text { Differenz }\end{array}$} & & & \\
\hline & & & & & Untere & Obere & & & \\
\hline $\begin{array}{l}\text { Paa- } \\
\text { ren } 1\end{array}$ & $\begin{array}{l}\text { Durchschnittliche Ener- } \\
\text { gieaufnahme in t1 an } \\
\text { Werktagen - } \\
\text { durchschnittliche Ener- } \\
\text { gieaufnahme in t1 am } \\
\text { Wochenende }\end{array}$ & -517 & 766 & 204 & -960 & -75 & $-2,52$ & 13 &, 025 \\
\hline $\begin{array}{l}\text { Paa- } \\
\text { ren } 2\end{array}$ & $\begin{array}{l}\text { Durchschnittliche Ener- } \\
\text { gieaufnahme in } \mathrm{t} 2 \text { an } \\
\text { Werktagen - } \\
\text { durchschnittliche Ener- } \\
\text { gieaufnahme in } \mathrm{t} 2 \mathrm{am} \\
\text { Wochenende }\end{array}$ & -152 & 936 & 250 & -692 & 388 &,- 608 & 13 &, 553 \\
\hline $\begin{array}{l}\text { Paa- } \\
\text { ren } 3\end{array}$ & $\begin{array}{l}\text { Durchschnittliche Ener- } \\
\text { gieaufnahme in t3 an } \\
\text { Werktagen - } \\
\text { durchschnittliche Ener- } \\
\text { gieaufnahme in } \mathrm{t} 3 \text { am } \\
\text { Wochenende }\end{array}$ & -87 & 1321 & 353 & -850 & 675 &,- 249 & 13 & ,807 \\
\hline $\begin{array}{l}\text { Paa- } \\
\text { ren } 4\end{array}$ & $\begin{array}{l}\text { Durchschnittliche Ener- } \\
\text { gieaufnahme in } \mathrm{t} 1 \text { an } \\
\text { Wochentagen - } \\
\text { durchschnittliche Ener- } \\
\text { gieaufnahme in } \mathrm{t} 2 \text { an } \\
\text { Wochentagen }\end{array}$ & 129 & 507 & 135 & -163 & 423 & ,956 & 13 & ,356 \\
\hline $\begin{array}{l}\text { Paa- } \\
\text { ren } 5\end{array}$ & $\begin{array}{l}\text { Durchschnittliche Ener- } \\
\text { gieaufnahme in } \mathrm{t} 1 \text { an } \\
\text { Wochentagen - } \\
\text { durchschnittliche Ener- } \\
\text { gieaufnahme in } \mathrm{t} 3 \text { an } \\
\text { Wochentagen }\end{array}$ & 217 & 718 & 192 & -197 & 631 & 1,13 & 13 & ,279 \\
\hline $\begin{array}{l}\text { Paa- } \\
\text { ren } 6\end{array}$ & $\begin{array}{l}\text { Durchschnittliche Ener- } \\
\text { gieaufnahme in } \mathrm{t} 2 \text { an } \\
\text { Wochentagen - } \\
\text { durchschnittliche Ener- } \\
\text { gieaufnahme in } \mathrm{t} 3 \text { an } \\
\text { Wochentagen }\end{array}$ & 87 & 348 & 93 & -114 & 288 & ,936 & 13 & ,366 \\
\hline $\begin{array}{l}\text { Paa- } \\
\text { ren } 7\end{array}$ & $\begin{array}{l}\text { Durchschnittliche Ener- } \\
\text { gieaufnahme in t1 am } \\
\text { Wochenende - } \\
\text { durchschnittliche Ener- } \\
\text { gieaufnahme in t } 2 \text { am } \\
\text { Wochenende }\end{array}$ & 495 & 1218 & 325 & -208 & 1199 & 1,52 & 13 &, 152 \\
\hline $\begin{array}{l}\text { Paa- } \\
\text { ren } 8\end{array}$ & $\begin{array}{l}\text { Durchschnittliche Ener- } \\
\text { gieaufnahme in t1 am } \\
\text { Wochenende - } \\
\text { durchschnittliche Ener- } \\
\text { gieaufnahme in t3 am } \\
\text { Wochenende }\end{array}$ & 647 & 1736 & 464 & -355 & 1649 & 1,39 & 13 &, 187 \\
\hline $\begin{array}{l}\text { Paa- } \\
\text { ren } 9\end{array}$ & $\begin{array}{l}\text { Durchschnittliche Ener- } \\
\text { gieaufnahme in t } 2 \text { am } \\
\text { Wochenende - } \\
\text { durchschnittliche Ener- } \\
\text { gieaufnahme in } \mathrm{t} 3 \text { am } \\
\text { Wochenende }\end{array}$ & 151 & 810 & 216 & -316 & 619 & ,699 & 13 & ,497 \\
\hline
\end{tabular}


Die folgenden Abbildungen veranschaulichen die prozentuale Zusammensetzung der Ernährung der Forstwirte zu allen drei Messzeitpunkten. Es werden die prozentualen Anteile der Kohlenhydrate, der Eiweiße und der Fette in den Diagrammen dargestellt. Zur besseren Vergleichbarkeit werden Diagramme zur optimalen Ernährung den Messergebnissen gegenübergestellt.

Für eine optimale Ernährung werden ein Kohlenhydratanteil von $60 \%$, ein Fettanteil von $30 \%$ und ein Eiweißanteil von $10 \%$ empfohlen (vgl. Kapitel 2). Zum Messzeitpunkt 1 beträgt der Anteil der Kohlenhydrate $45 \%$ der Energieaufnahme. $36 \%$ der Nahrung setzen sich aus Fett und $15 \%$ aus Eiweißen zusammen (vgl. Abbildung 11). Der Kohlenhydratanteil liegt somit deutlich unter dem empfohlenen Wert. Diese Differenz wird durch einen um $5 \%$ erhöhten Anteil an Eiweißen und Fetten ausgeglichen.

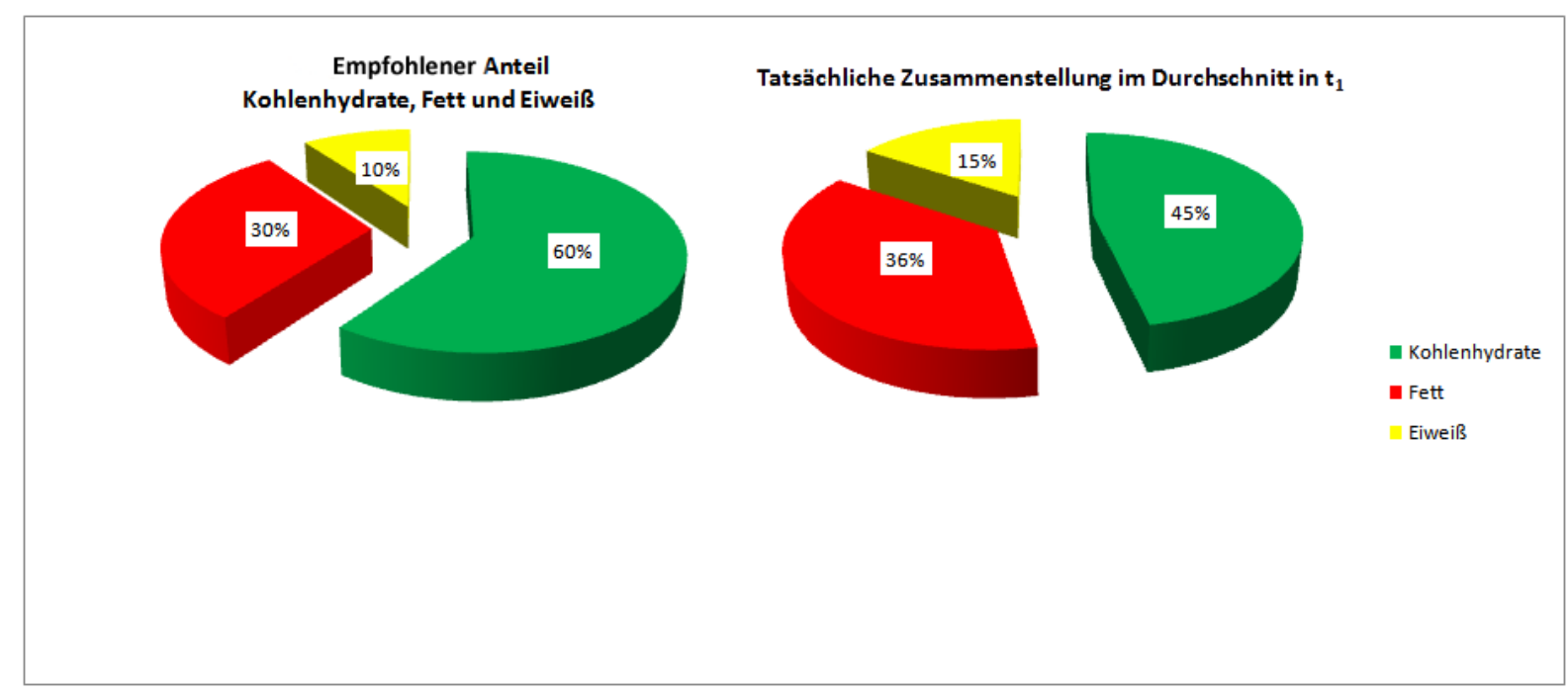

Abbildung 11: Empfohlene Ernährungszusammensetzung und Zusammensetzung der Ernährung in t1. 
Der Kohlenhydratanteil zu t2 beträgt $42 \%$, der Fettanteil steigt im Vergleich zu t1 auf $39 \%$ an und der Eiweißanteil macht $17 \%$ der Nahrungsaufnahme aus (vgl. Abbildung 12). Der Kohlenhydratanteil ist um $3 \%$ geringer als in t1. Der Fettanteil liegt $9 \%$ und der Eiweißanteil $7 \%$ über den empfohlenen Werten. Durch diese Verteilung ist der Fettanteil in t2 nahezu so groß wie der Kohlenhydratanteil.

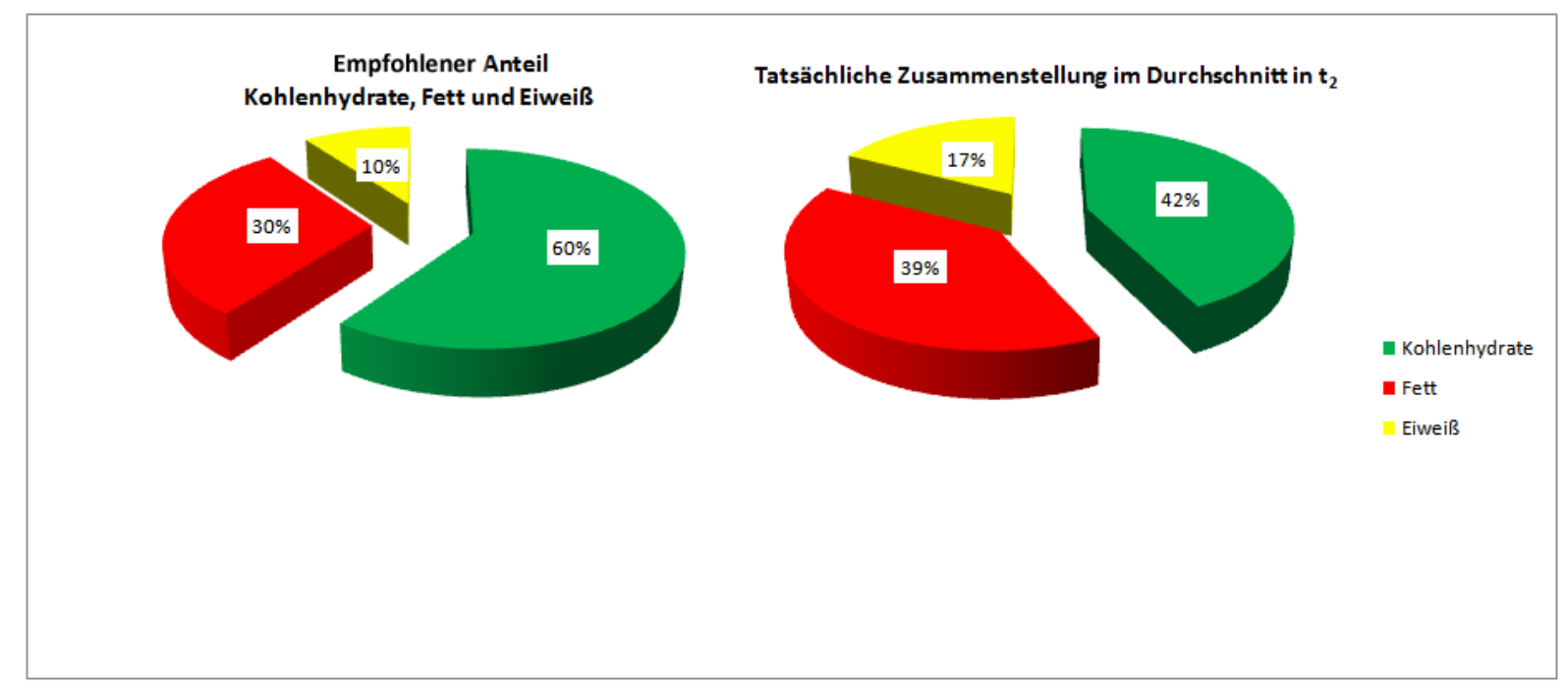

Abbildung 12: Empfohlene Ernährungszusammensetzung und Zusammensetzung der Ernährung in t2.

Die Energieaufnahme der Probanden in t3 setzt sich aus $43 \%$ Kohlenhydraten, $36 \%$ Fett und $16 \%$ Eiweißen zusammen (vgl. Abbildung 13). Auch in t3 liegt der Kohlenhydratanteil deutlich unter dem empfohlenen Wert, wohingegen der Fett- und der Eiweißanteil mit $6 \%$ über den empfohlenen Werten liegen.

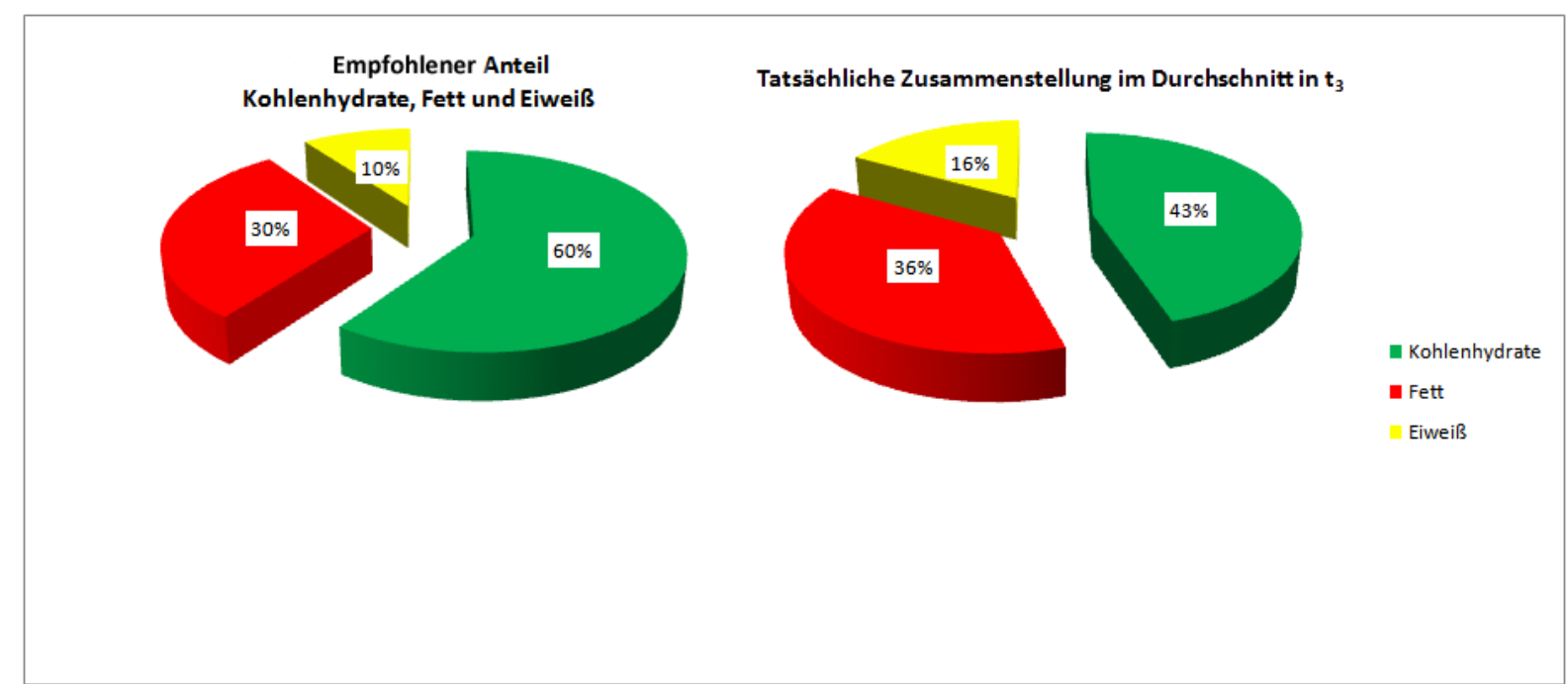

Abbildung 13: Empfohlene Ernährungszusammensetzung und Zusammensetzung der Ernährung in $\mathbf{t} 3$. 
Erkennbar ist, dass in $\mathrm{t} 2$ die größte Abweichung von der empfohlenen Zusammensetzung der Ernährung vorliegt.

Eine optimale, empfohlene Ernährung setzt sich zu $60 \%$ aus Kohlenhydraten, zu $10 \%$ aus Eiweiß und zu $30 \%$ aus Fett zusammen. Erhebungen zur tatsächlichen Ernährungszusammensetzung in der allgemeinen Bevölkerung, der so genannten Zivilisationskost, ergeben einen Kohlenhydratanteil von $35 \%$, einen Fettanteil von $20 \%$ und einen Eiweißanteil von $45 \%$. Da bei diesen Werten keine jahreszeitliche Differenzierung vorgenommen wird, werden diese Werte in Abbildung 14 den durchschnittlichen Anteilen (in t1, t2 und t3) der Stichprobe gegenübergestellt. Es ist festzustellen, dass die Forstwirte mit einer Differenz von $8 \%$ einen höheren Anteil an Kohlenhydraten zu sich nehmen. Darüber hinaus ist der Fettanteil der Ernährung der Forstwirte geringer als der Fettanteil der Bevölkerung. Der Eiweißanteil unterscheidet sich mit einem geringeren Wert bei den Forstwirten um $5 \%$.

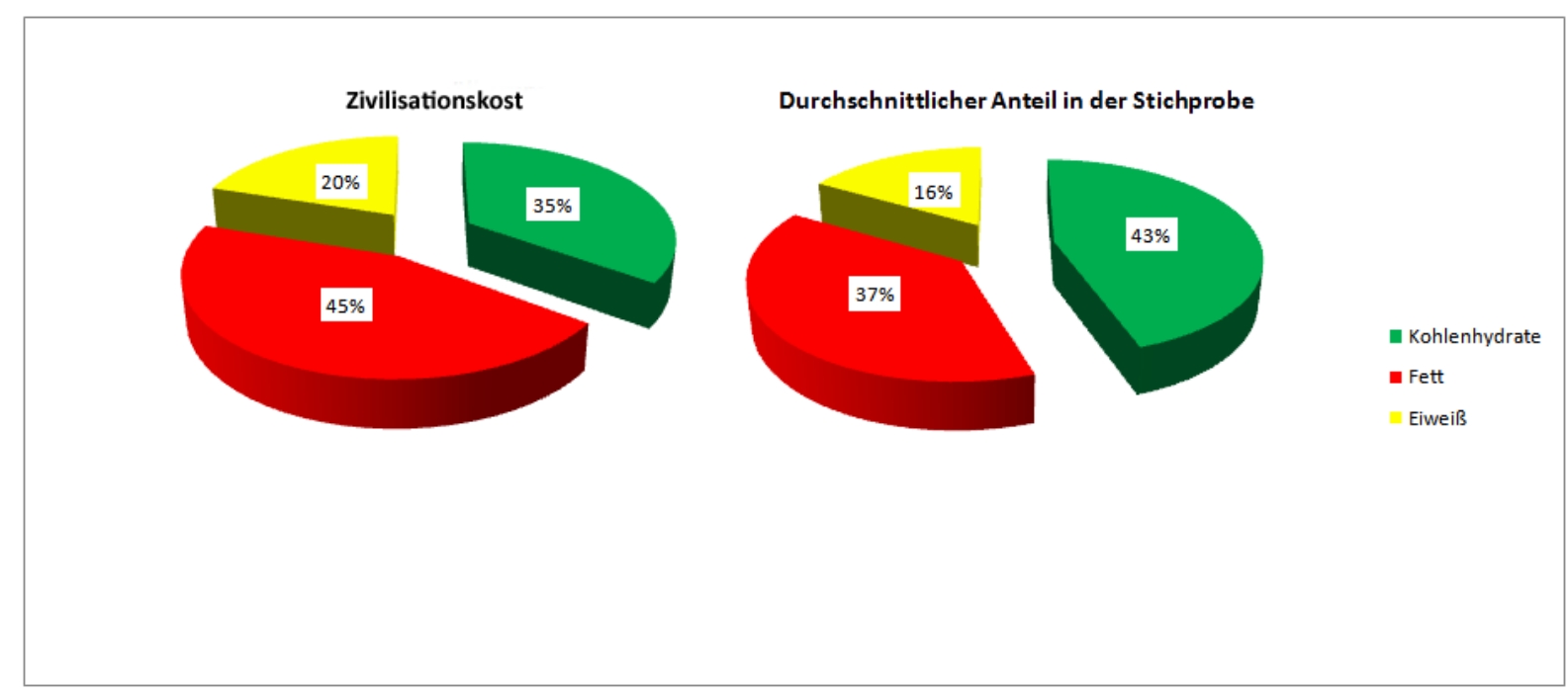

Abbildung 14: Zivilisationskost und durchschnittliche Ernährungsanteile in der Stichprobe.

Eine tabellarische Zusammenfassung der o. g. Ergebnisse (vgl. Tabelle 12) gibt eine Übersicht über die Nährstoffanteile in einer optimalen Ernährung, in der Zivilisationskost sowie in der Stichprobe in $\mathrm{t} 1, \mathrm{t} 2, \mathrm{t} 3$ und die gemittelten Werte der Stichprobe.

Tabelle 12: Überblick über die Zusammensetzung der Ernährung (in \%).

\begin{tabular}{|l|r|r|r|r|r|r|}
\hline \hline & \multicolumn{1}{|c|}{ optimal } & $\mathrm{t} 1$ & $\mathrm{t} 2$ & $\mathrm{t} 3$ & \multicolumn{1}{c|}{ MW } & Zivilisationskost \\
\hline Eiweiß & 10 & 15 & 17 & 16 & 16 & 20 \\
\hline Kohlenhydrate & 60 & 45 & 42 & 43 & 43 & 35 \\
\hline Fett & 30 & 36 & 39 & 36 & 37 & 45 \\
\hline \hline
\end{tabular}

Eine Übersicht über die Mittelwerte sowie die Minimal- und Maximalwerte und die Standardabweichungen bei der prozentualen Verteilung der Makronährstoffe gibt Tabelle 13. Der 
prozentuale Anteil der Kohlenhydrate erreicht mit $45 \%$ in $\mathrm{t} 1$ seinen Höchstwert. In t2 kann sowohl für den Fettanteil mit 38,5 \% als auch für den Eiweißanteil mit 16,6 \% der höchste Wert verzeichnet werden.

Grundsätzlich liegt der Fettanteil der Ernährung der Forstwirte mit $37 \%$ deutlich über den $30 \%$ des empfohlenen Fettanteils. Der Eiweißanteil übersteigt mit $16 \%$ um $6 \%$ den empfohlenen Wert und beim Kohlenhydratanteil liegen die $43 \%$ mit $17 \%$ unter dem empfohlenen Anteil. Der Vergleich mit der prozentualen Nahrungszusammensetzung der allgemeinen Bevölkerung hebt hervor, dass der Eiweiß- und der Fettanteil der Ernährung der Forstwirte deutlich unter den jeweiligen Anteilen der Zivilisationskost liegen. Im Bereich der Kohlenhydrate weist die Ernährung der Forstwirte einen höheren Anteil auf.

Tabelle 13: Auflistung der prozentualen Anteile von Kohlenhydraten, Fett und Eiweiß in t1-t3.

\begin{tabular}{|l|r|r|r|r|r|}
\hline \hline & $\mathrm{N}$ & Minimum & Maximum & Mittelwert & Standardabweichung \\
\hline Fettanteil der Ernährung in t1 (in \%) & 14 & 17,7 & 45,1 & 35,986 & 6,6152 \\
\hline Kohlenhydratanteil der Ernährung in t1 (in \%) & 14 & 37,7 & 60,0 & 45,100 & 5,6902 \\
\hline Eiweißanteil der Ernährung in t1 (in \%) & 14 & 11,9 & 18,3 & 15,221 & 1,7321 \\
\hline Fettanteil der Ernährung in t2 (in \%) & 14 & 24,9 & 47,4 & 38,550 & 5,5489 \\
\hline Kohlenhydratanteil der Ernährung in t2 (in \%) & 14 & 36,1 & 55,7 & 41,950 & 5,0482 \\
\hline Eiweißanteil der Ernährung in t2 (in \%) & 14 & 12,4 & 23,9 & 16,600 & 3,5444 \\
\hline Fettanteil der Ernährung in t3 (in \%) & 14 & 27,1 & 43,4 & 36,243 & 4,4999 \\
\hline Kohlenhydratanteil der Ernährung in t3 (in \%) & 14 & 30,3 & 56,4 & 43,221 & 7,4279 \\
\hline Eiweißanteil der Ernährung in t3 (in \%) & 14 & 12,7 & 25,3 & 16,400 & 3,3718 \\
\hline Gültige Werte (Listenweise) & 14 & & & & \\
\hline \hline
\end{tabular}


Aufgrund der hohen Diskrepanz zwischen Minimal- und Maximalwerten bei der prozentualen Verteilung der Nährstoffe (vgl. Tabelle 13), wird in den Abbildungen 15 bis 17 eine Einzeldarstellung der prozentualen Anteile vorgenommen. Die bestehende Differenz zu $100 \%$ liegt an den Mikronährstoffen, die in der Darstellung nicht berücksichtigt werden.

In 11 besteht bei FW08 ein deutlich geringerer Fettanteil. FW05 und FW13 weisen einen erhöhten Fettanteil auf Kosten des Kohlenhydratanteils auf.

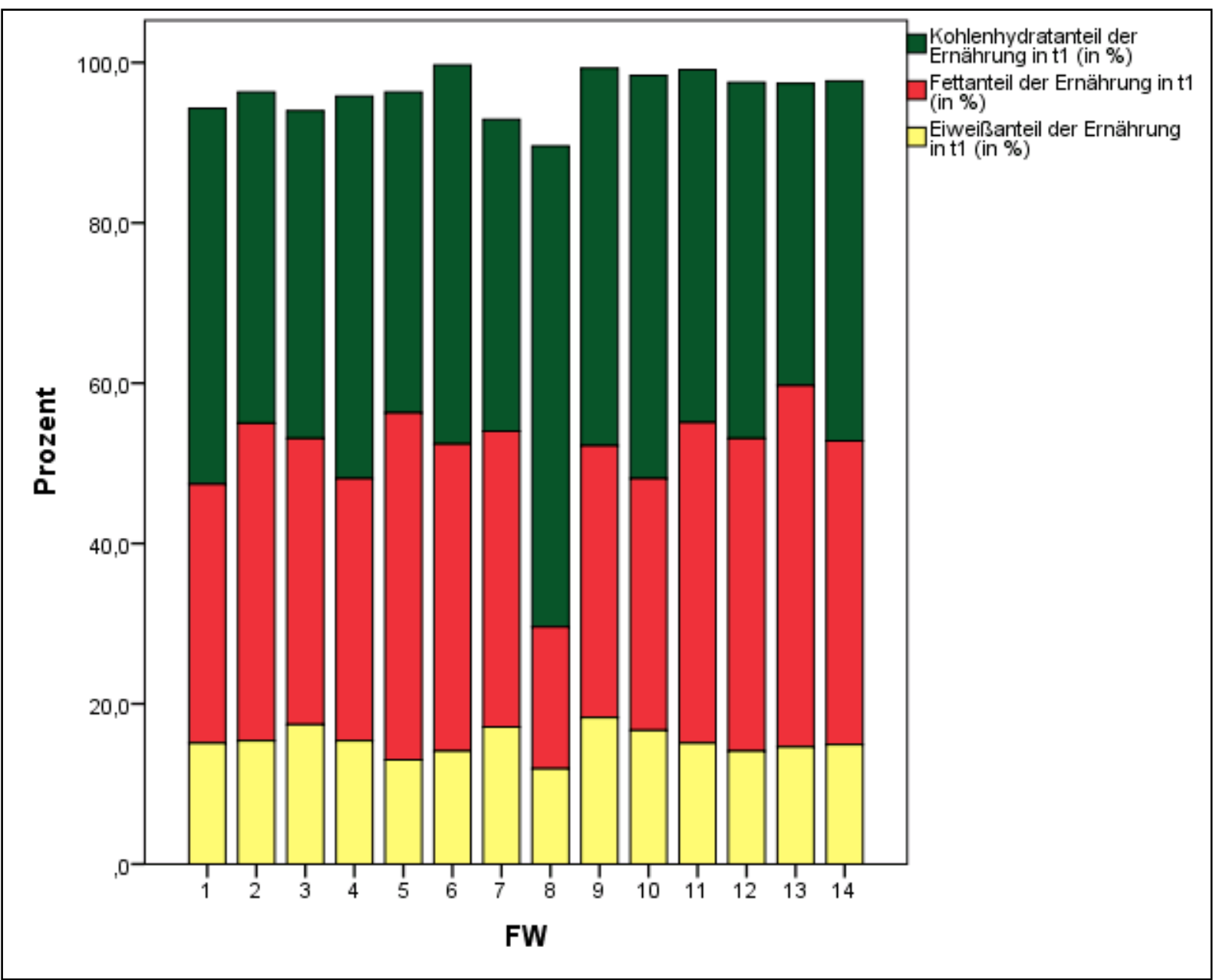

Abbildung 15: Durchschnittlicher Anteil von Kohlenhydraten, Fett und Eiweiß in t1 pro Person (in \%). 
Die Eiweißanteile in t2 betragen bei FW07, FW09 und FW10 mehr als $20 \%$. Ein geringer Fettanteil kann (ähnlich wie in t1) bei FW08 gemessen werden, der wiederum den höchsten Kohlenhydratanteil aufweist.

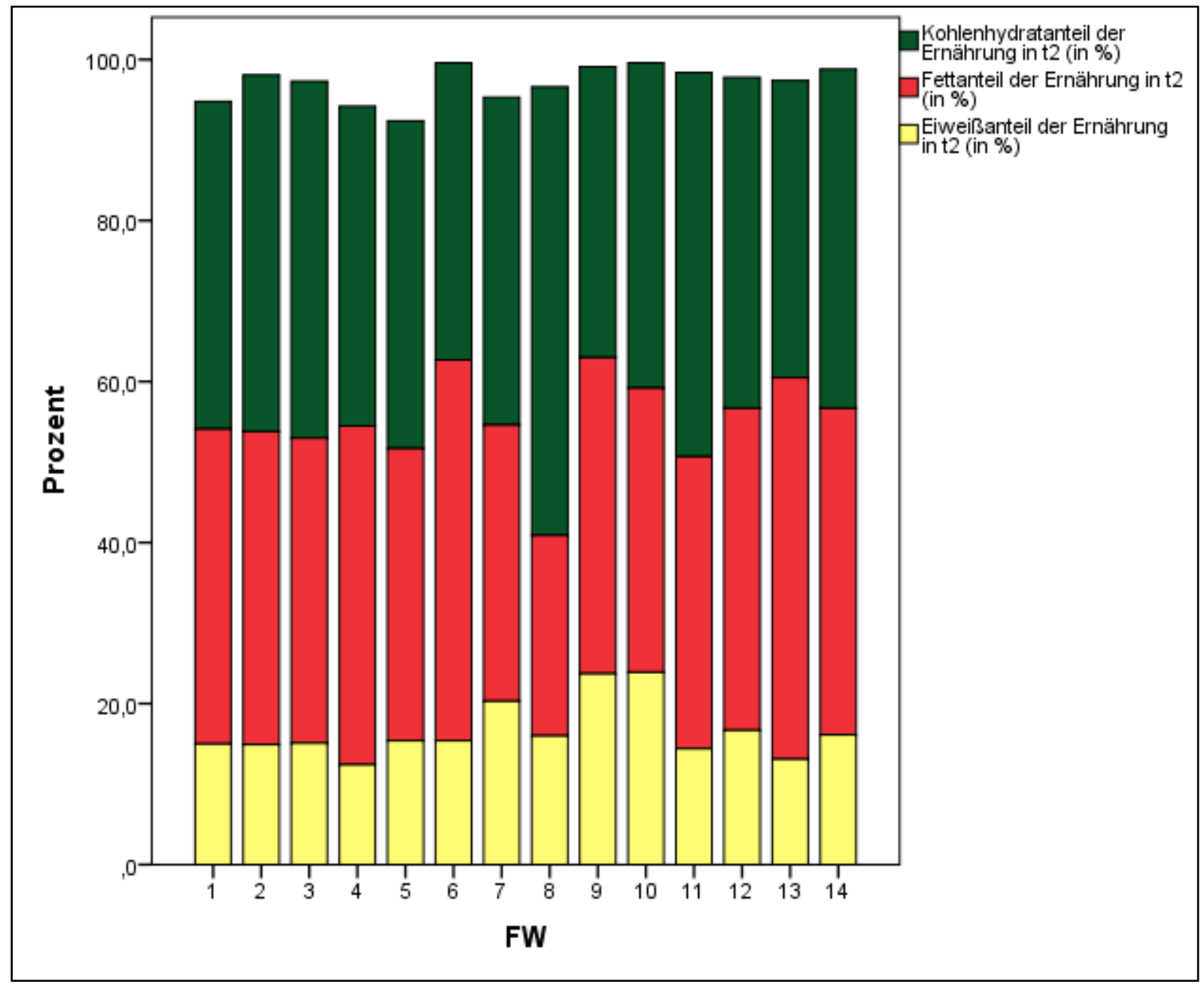

Abbildung 16: Durchschnittlicher Anteil von Kohlenhydraten, Fett und Eiweiß in t2 pro Person (in \%). 
Der Eiweißanteil ist auch in $\mathrm{t} 3$ bei FW07 und FW09 mit einem Wert von über $20 \%$ höher als bei den anderen Forstwirten. Der niedrige Fettanteil bei FW08 hebt sich in t3 geringer vom durchschnittlichen prozentualen Fettanteil der Stichprobe ab.

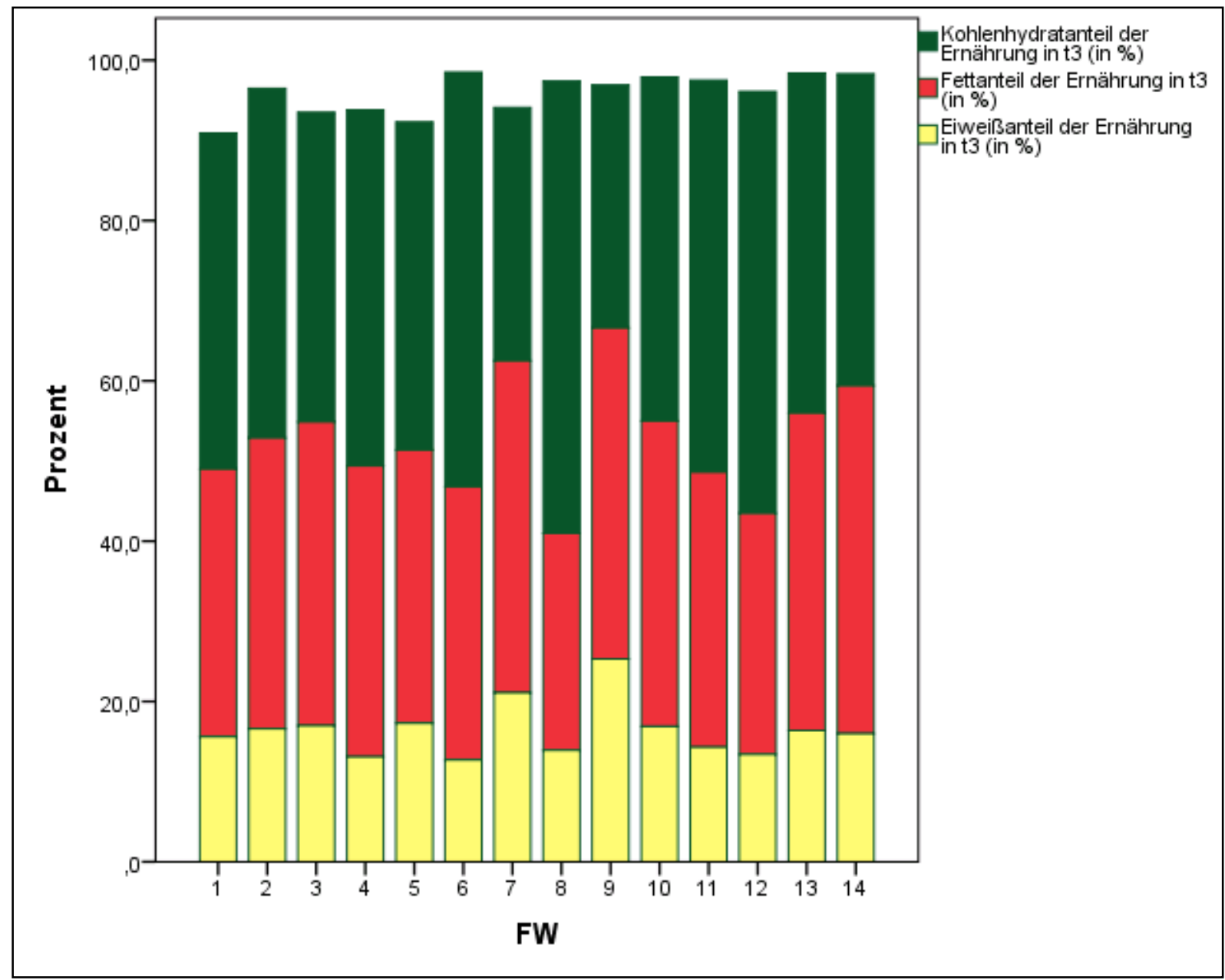

Abbildung 17: Durchschnittlicher Anteil von Kohlenhydraten, Fett und Eiweiß in t3 pro Person (in \%). 
Hinsichtlich der Flüssigkeitszufuhr werden im Folgenden der Wasseranteil in Liter und der Alkoholanteil in Gramm angegeben. Die Werte sind jeweils umgerechnet auf die Menge, die durchschnittlich an einem Tag von den Probanden konsumiert wird. Bei dieser Darstellung wird ebenfalls eine Unterteilung in Werktage (Montag bis Donnerstag) und Wochenende (Samstag und Sonntag) vorgenommen, um eventuelle Abweichungen zu erkennen.

Tabelle 14: Wasser- (in I) und Alkoholkonsum (in g) im Durchschnitt.

\begin{tabular}{|c|c|c|c|c|c|}
\hline & $\mathrm{N}$ & Minimum & Maximum & Mittelwert & Standardabweichung \\
\hline Flüssigkeitszufuhr in t1 an Werktagen & 14 & 2,2 & 6,1 & 3,617 & 1,0623 \\
\hline Alkoholkonsum in $\mathrm{t} 1$ an Werktagen & 14 &, 0 & 16,5 & 3,007 & 4,6061 \\
\hline Flüssigkeitszufuhr in $\mathrm{t} 1 \mathrm{am}$ Wochenende & 14 & 2,3 & 4,7 & 3,471 & ,8042 \\
\hline Alkoholkonsum in $\mathrm{t} 1 \mathrm{am}$ Wochenende & 14 &, 0 & 167,0 & 33,429 & 45,4041 \\
\hline Flüssigkeitszufuhr in $\mathrm{t} 2$ an Werktagen & 14 & 2,3 & 5,4 & 3,539 &, 8525 \\
\hline Alkoholkonsum in $\mathrm{t} 2$ an Werktagen & 14 &, 0 & 16,5 & 4,729 & 6,2887 \\
\hline Flüssigkeitszufuhr in $\mathrm{t} 2$ am Wochenende & 14 & 1,9 & 4,5 & 3,271 &, 7710 \\
\hline Alkoholkonsum in $\mathrm{t} 2 \mathrm{am}$ Wochenende & 14 &, 0 & 63,0 & 18,393 & 18,3210 \\
\hline Flüssigkeitszufuhr an $\mathrm{t} 3$ an Werktagen & 14 & 1,9 & 5,2 & 3,514 & ,9699 \\
\hline Alkoholkonsum in $\mathrm{t} 3$ an Werktagen & 14 &, 0 & 29,5 & 7,743 & 10,5060 \\
\hline Flüssigkeitszufuhr in $\mathrm{t} 3$ am Wochenende & 14 & 1,9 & 5,1 & 3,136 & 1,0135 \\
\hline Alkoholkonsum in $\mathrm{t} 3 \mathrm{am}$ Wochenende & 14 & 0 & 46,5 & 14,786 & 14,1771 \\
\hline Durchschnittliche Flüssigkeitszufuhr in t1 pro Tag & 14 & 2,3 & 5,5 & 3,528 &, 8378 \\
\hline Durchschnittlicher Alkoholkonsum in t1 pro Tag & 14 &, 0 & 58,9 & 13,683 & 16,6869 \\
\hline Durchschnittliche Flüssigkeitszufuhr in $\mathrm{t} 2$ pro Tag & 14 & 2,4 & 5,0 & 3,460 & ,7417 \\
\hline Durchschnittlicher Alkoholkonsum in $\mathrm{t} 2$ pro Tag & 14 &, 0 & 26,7 & 10,161 & 9,9522 \\
\hline Durchschnittliche Flüssigkeitszufuhr in $\mathrm{t} 3$ pro Tag & 14 & 2,1 & 5,3 & 3,455 & ,9846 \\
\hline Durchschnittlicher Alkoholkonsum in $\mathrm{t} 3$ pro Tag & 14 & 1,9 & 32,0 & 15,256 & 11,9564 \\
\hline Gültige Werte (Listenweise) & 14 & 14 & 14 & 14 & 14 \\
\hline
\end{tabular}

Bei der Flüssigkeitszufuhr fällt auf, dass in allen drei Messzeitpunkten die Zufuhr an Wochentagen leicht über der Zufuhr am Wochenende liegt. Ein Vergleich der Flüssigkeitszufuhr $\mathrm{zu}$ den einzelnen Messzeitpunkten ergibt mit Werten von ca. 3,5 1/ d eine recht konstante Flüssigkeitszufuhr.

Bei der Betrachtung der Alkoholwerte stechen in der deskriptiven Tabelle vor allem die hohen Alkoholwerte an den Wochenenden hervor. Hierbei muss zusätzlich bedacht werden, dass die Alkoholzufuhr der Freitage in der Berechnung keine Berücksichtigung findet. Insbesondere der hohe Wert am durchschnittlichen Wochenende im Winter ist mit 33,4 g Alkohol auffällig hoch. Ein Vergleich mit dem empfohlenen Grenzwert von 24 g/ d (BZGA 2011) zeigt auf, dass dieser Wert um 9,4 g Alkohol im Schnitt übertroffen wird. Im Einzelfall wird mit $167 \mathrm{~g} /$ d Alkohol in t1 am Wochenende der höchste Wert insgesamt erreicht. Der durchschnittliche 
Alkoholkonsum ist jedoch in $\mathrm{t} 3$ am Höchsten. Hier zeigt sich interessanterweise, dass im Gegensatz zu den vorherigen erfassten Zeitpunkten alle Probanden zumindest eine geringe Menge Alkohol zu sich nehmen.

Nach der Darstellung der Energieaufnahme und der prozentualen Zusammensetzung der Ernährung werden im nächsten Kapitel die Ergebnisse der Aktivitätsprotokolle zusammengefasst.

\subsection{Aktivitätsprotokoll}

Aus den Aktivitätsprotokollen lässt sich entnehmen, wie hoch der Energieumsatz der Probanden in den einzelnen Messzeitpunkten ist. Darüber hinaus lässt sich der Kalorienverbrauch in die Bereiche einteilen, in denen er stattfindet: Energieverbrauch in der Freizeit, bei sportlichen Aktivitäten und im Beruf.

Zunächst wird eine Betrachtung des allgemeinen Energieumsatzes vorgenommen. Tabelle 15 zeigt an, wie hoch der durchschnittliche Kalorienverbrauch pro Tag in den einzelnen Messzeitpunkten und an Wochentagen bzw. am Wochenende ist. Bei den Werten der Wochentage handelt es sich um die Werte von Montag bis Donnerstag, um eine Vergleichbarkeit mit den bisher dargestellten Werten zu ermöglichen und um den Freitag mit kürzerer Arbeitszeit und von den Wochentagen abweichenden Freizeitaktivitäten nicht in die Wertung einfließen zu lassen.

Tabelle 15: Energieumsatz in $\mathrm{t} 1, \mathrm{t} 2$ und $\mathrm{t} 3$ (in kcal).

\begin{tabular}{|l|r|r|r|r|r|}
\hline \hline & $\mathrm{N}$ & Minimum & Maximum & Mittelwert & Standardabweichung \\
\hline Durchschnittlicher Energieumsatz in t1 pro Tag & 14 & 3112 & 5142 & 3887,57 & 577,095 \\
\hline Durchschnittlicher Energieumsatz in t2 pro Tag & 14 & 3289 & 4922 & 4118,36 & 481,111 \\
\hline Durchschnittlicher Energieumsatz in t3 pro Tag & 14 & 3516 & 5145 & 3942,64 & 420,725 \\
\hline Energieumsatz in t1 an Werktagen pro Tag & 14 & 2661 & 4751 & 3480,50 & 618,802 \\
\hline Energieumsatz in t2 an Werktagen pro Tag & 14 & 3448 & 5818 & 4609,79 & 641,801 \\
\hline Energieumsatz in t3 an Werktagen pro Tag & 14 & 3672 & 6294 & 4383,14 & 678,335 \\
\hline Energieumsatz in t1 am Wochenende pro Tag & 14 & 2205 & 3351 & 2843,57 & 335,170 \\
\hline Energieumsatz in t2 am Wochenende pro Tag & 14 & 2222 & 4033 & 3064,64 & 485,027 \\
\hline Energieumsatz in t3 am Wochenende pro Tag & 14 & 2302 & 3525 & 2947,64 & 14 \\
\hline Gültige Werte (Listenweise) & 14 & 14 & 14 & & 376,527 \\
\hline \hline
\end{tabular}

Aus den Aktivitäten geht hervor, dass im Frühling durchschnittlich die meiste Aktivität gemessen wurde ( $\overline{\mathrm{x}}_{\text {Aktivität_t2 }}=4118.36$ ). Dieser Wert lässt sich auch zu einzelnen Wochentagen nachzeichnen. Die Aktivitäten sind generell an den Werktagen im Durchschnitt in jeder Zeitperiode deutlich höher als am Wochenende. Aufgrund der unterschiedlichen Energieumsätze 
der Forstwirte zu den unterschiedlichen Jahreszeiten und dadurch unterschiedlichen Tätigkeiten kann Hypothese 1 verifiziert werden.

Der Energieumsatz in der Freizeit wird ebenfalls für $\mathrm{t} 1, \mathrm{t} 2$ und $\mathrm{t} 3$ und für die Wochentage und die Wochenenden pro Tag angegeben (vgl. Tabelle 16).

Tabelle 16: Energieumsatz in der Freizeit in $\mathbf{t} 1, \mathrm{t} 2$ und $\mathrm{t} 3$ (in kcal).

\begin{tabular}{|l|r|r|r|r|r|}
\hline \hline & N & Minimum & Maximum & Mittelwert & Standardabweichung \\
\hline Freizeitenergieumsatz in t1 pro Tag & 14 & 1001 & 1931 & 1374,64 & 260,589 \\
\hline Freizeitenergieumsatz in t2 pro Tag & 14 & 1102 & 1706 & 1371,21 & 203,324 \\
\hline Freizeitenergieumsatz in t3 pro Tag & 14 & 915 & 1704 & 1398,36 & 230,952 \\
\hline Freizeitenergieumsatz am Wochenende in t1 pro Tag & 14 & 1370 & 2934 & 2182,71 & 391,519 \\
\hline Freizeitenergieumsatz am Wochenende in t2 pro Tag & 14 & 1401 & 2911 & 2144,29 & 482,961 \\
\hline Freizeitenergieumsatz am Wochenende in t3 pro Tag & 14 & 1321 & 2864 & 2131,86 & 469,121 \\
\hline Freizeitenergieumsatz an Werktagen in t1 pro Tag & 14 & 613 & 1487 & 999,07 & 229,666 \\
\hline Freizeitenergieumsatz an Werktagen in t2 pro Tag & 14 & 529 & 1306 & 988,86 & 198,886 \\
\hline Freizeitenergieumsatz an Werktagen in t3 pro Tag & 14 & 558 & 1398 & 1030,64 & 219,344 \\
\hline Gültige Werte (Listenweise) & 14 & 14 & 14 & 14 & 14 \\
\hline \hline
\end{tabular}

Aus den Mittelwerten lässt sich ablesen, dass der Energieumsatz durch Aktivität in der Freizeit nur geringfügig unterschiedlich ist. Die meiste Freizeitaktivität wird am Wochenende aufgenommen. Die geringste Aktivität ist im Winter und im Frühjahr von Montag bis Donnerstag zu verzeichnen. Alle Mittelwerte unterliegen keinen signifikanten Unterschieden (s. Anhang, Anlage 7).

Bei der Betrachtung der sportlichen Aktivität sind starke Schwankungen sowohl zwischen den Messzeitpunkten als auch zwischen den Probanden zu erkennen (vgl. Tabelle 17).

Tabelle 17: Energieumsatz durch sportliche Aktivität (in kcal).

\begin{tabular}{|l|r|r|r|r|r|}
\hline \hline & $\mathrm{N}$ & Minimum & Maximum & Mittelwert & Standardabweichung \\
\hline Energieumsatz durch Sport in t1 pro Tag & 14 & 70 & 316 & 172,36 & 93,789 \\
\hline Energieumsatz durch Sport in t2 pro Tag & 14 & 0 & 732 & 208,93 & 238,679 \\
\hline Energieumsatz durch Sport in t3 pro Tag & 14 & 0 & 498 & 180,00 & 168,716 \\
\hline $\begin{array}{l}\text { Energieumsatz durch Sport am Wochenende in t1 pro } \\
\text { Tag }\end{array}$ & 14 & 0 & 330 & 23,57 & 88,196 \\
\hline $\begin{array}{l}\text { Energieumsatz durch Sport am Wochenende in t2 pro } \\
\text { Tag }\end{array}$ & 14 & 0 & 1780 & 257,14 & 580,520 \\
\hline $\begin{array}{l}\text { Energieumsatz durch Sport am Wochenende in t3 pro } \\
\text { Tag }\end{array}$ & 14 & 0 & 608 & 156,14 & 245,072 \\
\hline $\begin{array}{l}\text { Energieumsatz durch Sport an Werktagen in t1 pro } \\
\text { Tag }\end{array}$ & 14 & 122 & 516 & 248,71 & 129,154 \\
\hline $\begin{array}{l}\text { Energieumsatz durch Sport an Werktagen in t2 pro } \\
\text { Tag }\end{array}$ & 14 & 0 & 426 & 197,14 & 148,475 \\
\hline $\begin{array}{l}\text { Energieumsatz durch Sport an Werktagen in t3 pro } \\
\text { Tag }\end{array}$ & 14 & 0 & 548 & 197,57 & 169,268 \\
\hline Gültige Werte (Listenweise) & 14 & 14 & 14 & & 14 \\
\hline \hline
\end{tabular}


Während im Winter alle Probanden z. T. geringfügig Sport treiben, wobei der meiste Sport mit $248 \mathrm{kcal}$ werktags betrieben wird, ist der Mittelwert im Winter aber insgesamt am Geringsten. Im Frühjahr scheint die Sportaktivität am höchsten zu sein.

Versucht man aus den gemessenen Temperaturen Durchschnittstemperaturen für vormittags und nachmittags zu generieren, zeigt sich nur eine tendenzielle Korrelation zwischen der Temperatur vormittags und der Sportaktivität im Sommer (s. Anhang, Anlage 7). Ein Vergleich mit dem Wochenende ist hier unnötig, da nur Temperaturdaten von den jeweiligen fünf Werktagen erfasst wurden. Signifikante Unterschiede zeigen sich hier nicht.

Der Energieumsatz während beruflicher Tätigkeit wird zum einen durch die Höhe des Energieverbrauchs bei der gemessenen Tätigkeit und zum anderen durch die Gestaltung des Arbeitstages beeinflusst. Äußere Bedingungen können z. B. verhindern, dass die Forstwirte über die gesamte Arbeitszeit eine Tätigkeit ausführen. In Tabelle 18 werden durchschnittliche Energieumsätze pro Tag von den Tagen Montag bis Donnerstag aufgeführt.

Tabelle 18: Energieumsatz im Beruf (in kcal).

\begin{tabular}{|l|r|r|r|r|r|}
\hline \hline & $\mathrm{N}$ & Minimum & Maximum & Mittelwert & Standardabweichung \\
\hline Energieumsatz im Beruf in t1 pro Tag & 14 & 1553 & 4250 & 2581,93 & 769,147 \\
\hline Energieumsatz im Beruf in t2 pro Tag & 14 & 2091 & 4342 & 2929,36 & 579,262 \\
\hline Energieumsatz im Beruf in t3 pro Tag & 14 & 1786 & 4464 & 2673,14 & 671,520 \\
\hline Gültige Werte (Listenweise) & 14 & 14 & 14 & 14 & 14 \\
\hline \hline
\end{tabular}

Die berufliche Aktivität der Forstwirte ist in t2 am Höchsten (2929 kcal). Auffällig ist die große Diskrepanz zwischen dem niedrigsten Minimalwert in t1 mit $1553 \mathrm{kcal}$ und dem höchsten Maximalwert in t3 mit $4464 \mathrm{kcal}$. Signifikante Unterschiede zeigen sich im T-Test nicht (s. Anhang, Anlage 7). 


\section{4 Äußere Bedingungen}

Bei den äußeren Bedingungen als Einflussfaktoren werden aufgrund der Analyse des Anforderungsprofils Forstwirt Temperatur, Hangneigung und Bodenbewuchs als relevant erachtet. Zunächst werden in Tabelle 19 die äußeren Bedingungen deskriptiv dargestellt. Hierzu werden die Angaben der Protokolle der Wochentage Montag bis Donnerstag gemittelt, so dass zum einen ein Durchschnittswert, zum anderen Minimal- und Maximalwerte betrachtet werden.

Tabelle 19: Deskriptive Statistik der äußeren Bedingungen.

\begin{tabular}{|l|r|r|r|r|r|}
\hline \hline & $\mathrm{N}$ & Minimum & Maximum & Mittelwert & Standardabweichung \\
\hline Behinderung in t1 & 14 &, 00 & 2,25 &, 8214 &, 86285 \\
\hline Hangneigung in t1 & 14 &, 00 & 2,25 & 1,1429 &, 81874 \\
\hline Behinderung in t2 & 14 &, 00 & 1,50 &, 5893 &, 55128 \\
\hline Hangneigung in t2 & 14 &, 00 & 2,00 &, 6964 &, 67353 \\
\hline Behinderung in t3 & 14 &, 00 & 3,00 & 1,2143 &, 92952 \\
\hline Hangneigung in t3 & 14 &, 00 & 3,00 &, 9464 &, 94655 \\
\hline Gültige Werte (Listenweise) & 14 & 14 & 14 & 14 & 14 \\
\hline \hline
\end{tabular}

Die Tabelle spiegelt die unterschiedlichen Bedingungen bei der Tätigkeit der Forstwirte wider, was besonders in $\mathrm{t} 3$ durch die Minimal- und Maximalwerte verdeutlicht wird. Sowohl die Behinderung differiert hier von keiner bis zu sehr starker Behinderung als auch die Hangneigung, die von keiner bis zu einer starken Hangneigung differiert. Betrachtet man die Mittelwerte, so kann die Aussage getroffen werden, dass im Erhebungszeitraum in t1 im Durchschnitt eine mittlere Behinderung und eine leichte Hangneigung, in t2 eine geringe Behinderung und keine bis eine leichte Hangneigung und in $\mathrm{t} 3$ eine mittlere bis starke Behinderung und eine leichte Hangneigung bestanden.

Um den Einfluss der äußeren Bedingungen auf den Energieumsatz und den Sauerstoffverbrauch zu erfassen, wird in Tabelle 20 mittels Korrelation dieser Zusammenhang berechnet. Die Korrelationen werden in allen drei Messzeitpunkten zu den unterschiedlichen Tätigkeiten der Forstwirte vorgenommen, wobei die Temperatur, die Hangneigung und die Behinderung im Gelände Berücksichtigung finden. Grundlage für die Berechnung sind die Werte der Spiroergometrie und die äußeren Bedingungen, die bei der Messung der beruflichen Tätigkeiten vorherrschten. 
Tabelle 20: Korrelation Energieumsatz und Sauerstoffverbrauch - äußere Bedingungen.

\begin{tabular}{|c|c|c|c|c|c|c|c|}
\hline & & $\begin{array}{l}\text { Sauerstoffver- } \\
\text { brauch }\left(t_{1}\right)\end{array}$ & $\begin{array}{l}\text { Relativer } \\
\text { Energieum- } \\
\text { satz }\left(t_{1}\right)\end{array}$ & $\begin{array}{c}\text { Sauerstoffver- } \\
\text { brauch Belastung } \\
\left(\mathrm{t}_{2}\right)\end{array}$ & $\begin{array}{l}\text { Relativer } \\
\text { Energieum- } \\
\text { satz }\left(\mathrm{t}_{2}\right)\end{array}$ & $\begin{array}{c}\text { Sauerstoffver- } \\
\text { brauch Belastung } \\
\left(\mathrm{t}_{3}\right)\end{array}$ & $\begin{array}{l}\text { Relativer } \\
\text { Energieum- } \\
\text { satz }\left(\mathrm{t}_{3}\right)\end{array}$ \\
\hline \multirow{3}{*}{$\begin{array}{l}\text { Wie hoch ist } \\
\text { die Tempera- } \\
\text { tur? t1 } \mid \text { t2 } \\
\text { t3 }\end{array}$} & $\begin{array}{l}\text { Korrelation nach } \\
\text { Pearson }\end{array}$ & ,094 &, 185 & ,086 &, 104 &,- 148 &,- 183 \\
\hline & Signifikanz (2-seitig) &, 749 &, 525 & ,769 & ,724 & ,613 &, 532 \\
\hline & $\mathrm{N}$ & 14 & 14 & 14 & 14 & 14 & 14 \\
\hline \multirow{3}{*}{$\begin{array}{l}\text { Wie stark ist } \\
\text { die Hangnei- } \\
\text { gung? t1 } \mid \text { t2 } \\
\text { t3 }\end{array}$} & $\begin{array}{l}\text { Korrelationskoeffi- } \\
\text { zient nach Kendall }\end{array}$ & ,047 &, 172 & ,379 & ,379 & ,337 & ,372 \\
\hline & Sig. (2-seitig) & ,841 & ,463 & ,095 & ,095 &, 142 &, 105 \\
\hline & $\mathrm{N}$ & 14 & 14 & 14 & 14 & 14 & 14 \\
\hline \multirow{3}{*}{$\begin{array}{l}\text { Wie stark ist } \\
\text { die Behinde- } \\
\text { rung im } \\
\text { Gelände? } \\
\text { t1 | t2 t3 }\end{array}$} & $\begin{array}{l}\text { Korrelationskoeffi- } \\
\text { zient nach Kendall }\end{array}$ &, 151 &,- 091 & ,328 & ,391 & ,568 & ,541 \\
\hline & Sig. (2-seitig) & ,519 & ,699 & ,162 & ,096 & 010 & ,014 \\
\hline & $\mathrm{N}$ & 14 & 14 & 14 & 14 & 14 & 14 \\
\hline
\end{tabular}

$\mathrm{p} \leq 0,1$ tendenzielle Signifikanz

$\mathrm{p} \leq 0,01$ Signifikanz

Die Temperatur zeigt zu keinem Messzeitpunkt einen signifikanten Zusammenhang (Irrtumswahrscheinlichkeit <0,1). Die Hangneigung wirkt positiv auf den Sauerstoffverbrauch und auf den relativen Energieumsatz in t2. Das bedeutet, dass eine stärkere Hangneigung einen höheren Sauerstoffverbrauch und einen größeren relativen Energieumsatz bedingt. In t1 und $\mathrm{t} 3$ zeigt die Hangneigung keine Effekte. Die Stärke der Behinderung korreliert jeweils positiv mit dem relativen Energieumsatz in $\mathrm{t} 2$, dem Sauerstoffverbrauch in $\mathrm{t} 3$ und dem relativen Energieumsatz in t3. Je stärker die Behinderung ist, desto größer ist der relative Energieumsatz bzw. der Sauerstoffverbrauch bei Belastung. Alle erwähnten Effekte haben eine Stärke von $>|0,350|^{14}$ (mittlerer bis teilweise starker (> $\left.|0,5|\right)$ Zusammenhang).

\subsection{Indirekte Kalorimetrie}

Eine Datenerhebung mittels indirekter Kalorimetrie wurde bei allen Probanden in allen drei Messzeitpunkten während der typischen beruflichen Tätigkeit vorgenommen. Darüber hinaus wurden bei vier Forstwirten (FW01, FW03, FW04, FW05) anhand einer Fahrradspiroergometrie Daten erhoben, die eine Vergleichbarkeit der erhobenen Werte mit Werten, die bei bestimmten Leistungen in Watt erreicht werden, ermöglichen. Für die Auswertung der Analyseergebnisse werden im Folgenden die Sauerstoffaufnahme, der RQ und der Energieumsatz betrachtet.

\footnotetext{
${ }^{14}$ Die doppelten Balken stehen für Betrag, da die Korrelation nur positiv interpretiert wird, gleichgültig ob die Effekte negativ korrelieren.
} 
In der Literatur wird der Energieumsatz sowohl über die Sauerstoffaufnahme $\mathrm{VO}_{2} \mathrm{in} \mathrm{ml} / \mathrm{min}$ als auch über den Kalorienverbrauch in kcal/ min angegeben. Um in dieser Arbeit diese Werte miteinander vergleichen zu können, werden die Werte mit dem kalorischen Äquivalent (KÄ) in eine gemeinsame Einheit umgerechnet (vgl. Kapitel 3). Tabelle 21 gibt einen Überblick über die zugrunde gelegte Nahrungszusammensetzung und die kalorischen Äquivalente.

Tabelle 21: Kalorische Äquivalente für die Umrechnung des Energieumsatzes.

\begin{tabular}{|c|r|r|r|r|r|}
\hline \hline & Kohlenhydrate & Fett & Eiweiß & \multicolumn{1}{|c|}{$\mathrm{A}$ in KJ/1 $\mathrm{O}_{2}$} & $\mathrm{KÄ}$ in kcal/ $1 \mathrm{O}_{2}$ \\
\hline Empfohlene Kost & 60 & 30 & 10 & 20,39 & 4,877 \\
\hline Zivilisationskost & 35 & 45 & 20 & 19,975 & 4,778 \\
\hline $\mathrm{t} 1$ & 45 & 36 & 15 & 19,362 & 4,632 \\
\hline $\mathrm{t} 2$ & 42 & 39 & 17 & 19,108 & 4,571 \\
\hline $\mathrm{t} 3$ & 43 & 36 & 16 & 19,13 & 4,576 \\
\hline \hline
\end{tabular}

Da die Nahrungszusammensetzung für die der Literatur entnommenen Werte nicht bekannt ist, wird ein $\mathrm{K} \ddot{\mathrm{A}}$ von 4,8 kcal/ $1 \mathrm{O}_{2}$ eingesetzt, welches den Mittelwert vom KÄ der Zivilisationskost und vom KÄ der empfohlenen Nahrungszusammensetzung bildet. Für die Umrechnung der Energieumsätze der Probanden der vorliegenden Studie wird das KÄ mit Hilfe der erhobenen Nahrungszusammensetzung der Probanden in den drei Messzeitpunkten errechnet, wobei mit dem gemittelten Wert in Höhe von $4,6 \mathrm{kcal} / 1 \mathrm{O}_{2}$ bei der Umrechnung gerechnet wird.

\subsubsection{Spiroergometrie}

Die spiroergometrische Untersuchung wurde in drei Messzeitpunkten vorgenommen, welche während der Arbeitszeit der Forstwirte stattfand. Es wurde bei der Messung die Tätigkeit ausgeführt, die zum jeweiligen Messzeitpunkt hauptsächlich über mehrere Wochen ausgeführt wurde. In Tabelle 22 werden die Ergebnisse der spiroergometrischen Untersuchung der drei Messzeitpunkte deskriptiv dargestellt. 
Tabelle 22: Deskriptive Statistik Spiroergometrie

(Sauerstoffaufnahme in 1/min, RQ, relativer Energieumsatz in kcal/ kg KG/ min) bei beruflicher Tätigkeit.

\begin{tabular}{|l|r|r|r|r|r|}
\hline \hline & N & Minimum & Maximum & Mittelwert & Standardabweichung \\
\hline Sauerstoffaufnahme bei Belastung in t1 & 14 & 1,58 & 2,48 & 2,0381 &, 30715 \\
\hline Sauerstoffaufnahme bei Belastung in t2 & 14 & 1,07 & 2,69 & 1,7612 &, 38050 \\
\hline Sauerstoffaufnahme bei Belastung in t3 & 14 & 1,21 & 2,39 & 1,6313 &, 31055 \\
\hline RQ in t1 & 14 &, 74 &, 97 &, 8450 &, 07955 \\
\hline RQ in t2 & 14 &, 82 & 1,08 &, 9139 &, 07658 \\
\hline RQ in t3 & 14 &, 77 & 1,06 &, 8929 &, 07760 \\
\hline Absoluter Energieumsatz in t1 & 14 & 7,68 & 11,81 & 9,8404 & 1,43883 \\
\hline Absoluter Energieumsatz in t2 & 14 & 5,34 & 13,35 & 8,6564 & 1,87181 \\
\hline Absoluter Energieumsatz in t3 & 14 & 5,88 & 11,88 & 7,9852 & 1,54217 \\
\hline Relativer Energieumsatz in t1 & 14 &, 09 &, 14 &, 1147 &, 01702 \\
\hline Relativer Energieumsatz in t2 & 14 &, 06 &, 14 &, 0997 &, 02499 \\
\hline Relativer Energieumsatz in t3 & 14 &, 06 &, 12 &, 0921 &, 02061 \\
\hline Gültige Werte (Listenweise) & 14 & 14 & 14 & 14 & 14 \\
\hline \hline
\end{tabular}

Die Sauerstoffaufnahme bei Belastung hat sich im Verlauf von 2,04 1/ min auf 1,63 1/ min im Mittel reduziert, wobei die minimalen und maximalen Werte Schwankungen unterliegen. Der respiratorische Quotient zeigt dagegen im Durchschnitt erst einen größeren Anstieg und dann eine kleinere Abschwächung. Der RQ beträgt in t1 0,84, in t2 0,91 und in t3 0,89. Der relative Energieumsatz sinkt im Verlauf im Durchschnitt, was auch an den minimalen und maximalen Werten zu erkennen ist. Beträgt der relative Energieumsatz in t1 $0,11 \mathrm{kcal} / \mathrm{kg} \mathrm{KG} / \mathrm{min}$, so ist dieser Wert geringer in t2 bzw. t3 mit einem Wert von 0,099 kcal/ kg KG/ min bzw. $0,092 \mathrm{kcal} / \mathrm{kg} \mathrm{KG} / \mathrm{min}$. Der absolute Energieumsatz beträgt entsprechend in t1 9,8 kcal, in t2 $8,6 \mathrm{kcal}$ und in $\mathrm{t} 37,9 \mathrm{kcal}$. 
Um eine individuellere Analyse der spiroergometrischen Daten zu ermöglichen, bilden die folgenden drei Diagramme die Einzelwerte des Sauerstoffverbrauchs, des Respiratorischen Quotienten und des relativen Energieumsatzes ab (vgl. Abbildungen 18-20).

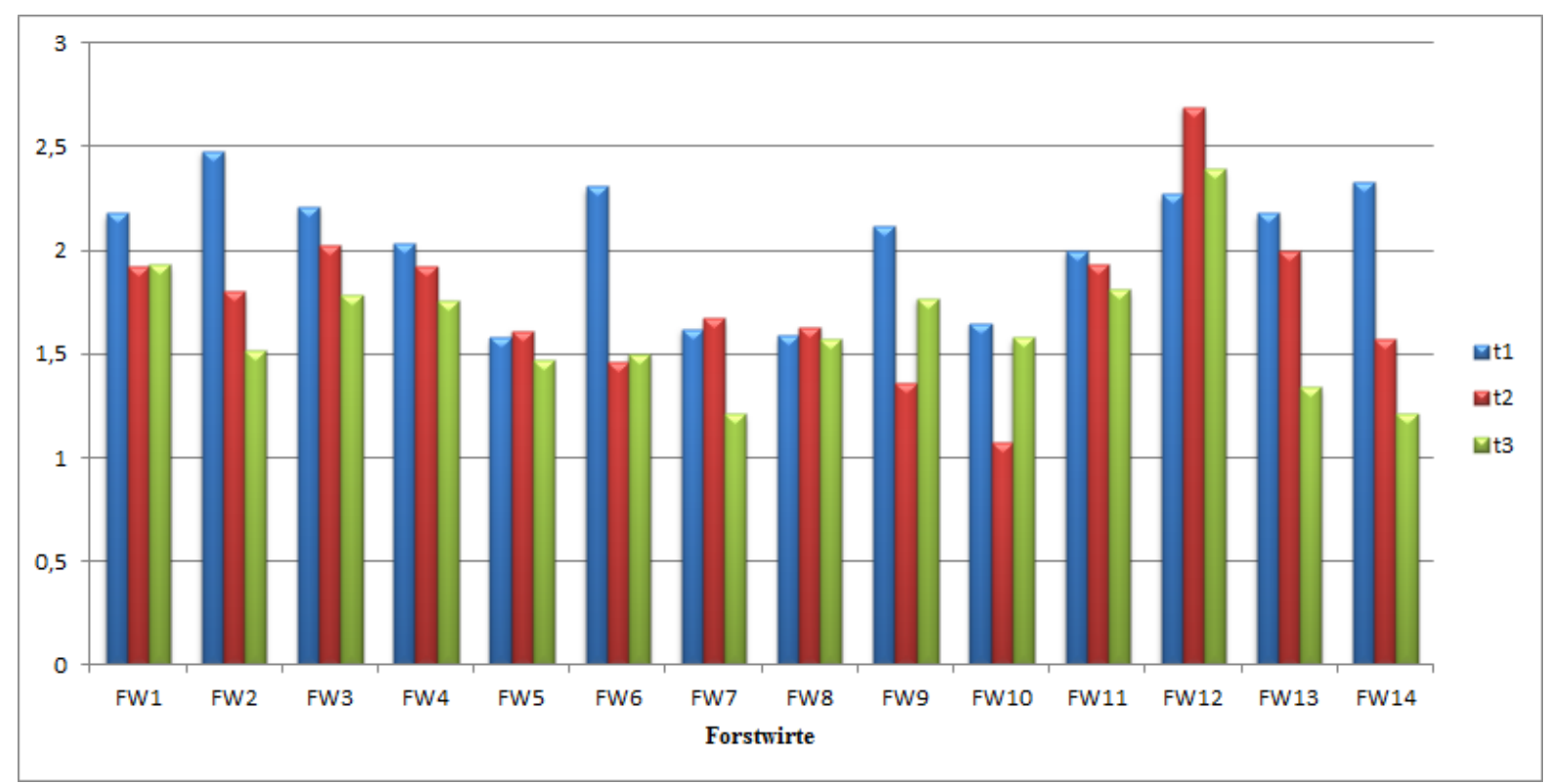

Abbildung 18: Sauerstoffaufnahme in l/ min bei Spiroergometrie.

FW02 (2,5 1/ min) weist in t1 die höchste, FW05 (1,6 1/ min) die niedrigste Sauerstoffaufnahme auf. In $\mathrm{t} 2$ und $\mathrm{t} 3$ erreicht FW12 die höchste Sauerstoffaufnahme mit 2,7 l/ min bzw. 2,4 1/ min. Die niedrigste Sauerstoffaufnahme erreicht in t2 FW10 mit 1,1 1/ min und in t3 FW14 mit 1,25 l/ min. Die Werte differieren somit in $\mathrm{t} 1 \mathrm{um} 0,9 \mathrm{l} / \mathrm{min}$, in $\mathrm{t} 2 \mathrm{um} \mathrm{1,6} \mathrm{l} / \mathrm{min}$ und in $\mathrm{t} 3 \mathrm{um} 1,15 \mathrm{l} / \mathrm{min}$.

Bei fünf Forstwirten (FW02, FW06, FW09, FW13, FW14) ist eine stärkere Differenz der Sauerstoffaufnahme in den drei Messzeitpunkten sichtbar, wobei der Wert in t1 (bei der Holzernte) bei allen am höchsten ausfällt. FW08 zeigt mit ca. 1,6 1/ min die konstanteste Sauerstoffaufnahme in allen drei Messzeitpunkten auf.

In Abbildung 20 wird der relative Energieumsatz in $\mathrm{kcal} / \mathrm{kg} \mathrm{KG} / \mathrm{min}$ dargestellt, um den Energieumsatz unabhängig von der Körperkomposition betrachten zu können. Hervorzuheben sind FW 04, FW05, FW08 und FW11, die einen nahezu konstanten relativen Energieumsatz in allen drei Messzeitpunkten aufweisen. Eine große Differenz bei den jeweiligen Werten ist bei FW02, FW13 und FW14 festzustellen mit einer maximalen Differenz von 0,07 kcal/ kg KG/ min (FW14), wobei der höchste Wert in t1 (motormanuelle Holzernte) und der niedrigste Wert in t3 (FW02: Jungwuchspflege; FW13 und 14: motormanuelle Holzernte) erreicht wird. Bei FW09 und FW10 liegt in $\mathrm{t} 2$ ein niedrigerer Wert vor, der bei der Arbeit mit dem Pflanzlochbohrer gemessen wurde. 


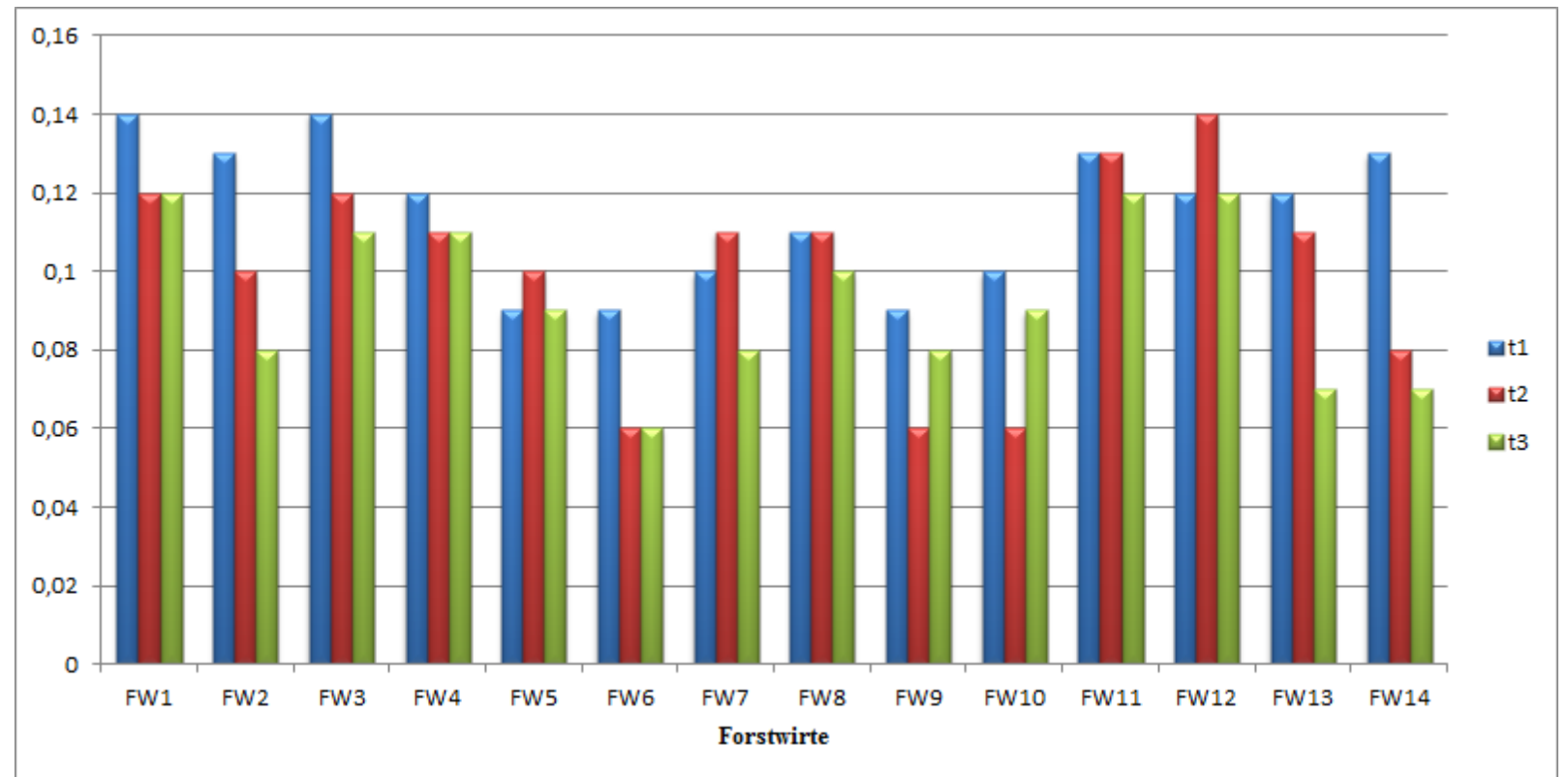

Abbildung 19: Relativer Energieumsatz in kcal/ kg KG/ min bei Spiroergometrie.

Anhand der Darstellung der RQ-Werte (vgl. Abbildung 19) werden drei Werte deutlich, die knapp über 1,0 liegen (FW05 in t2, FW10 in t2 und FW08 in t3). Alle anderen Werte liegen im Bereich um 0,85 mit Abweichungen sowohl nach oben als auch nach unten. Auffällig ist auch hier die Individualität der Werte. Die Forstwirte FW02, FW03, FW04 und FW09 weisen in allen Messzeitpunkten einen ähnlichen RQ auf, wohingegen die Differenz bei FW06, FW10, FW11 und FW12 am höchsten ist und ca. 0,2 beträgt.

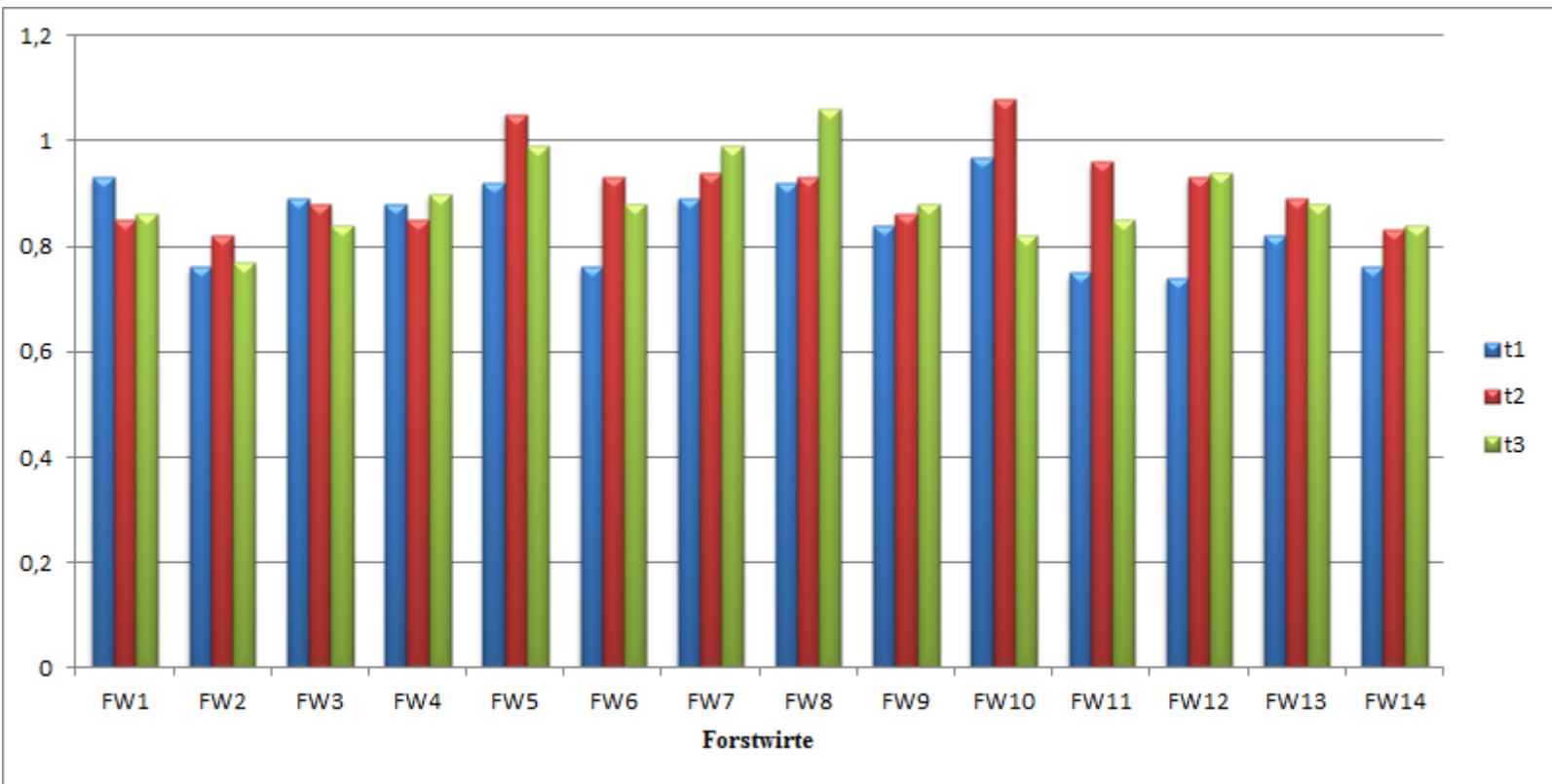

Abbildung 20: RQ bei Spiroergometrie. 
Im Paarvergleich ergeben sich signifikante Unterschiede zwischen der Sauerstoffaufnahme in $\mathrm{t} 1$ und in $\mathrm{t} 2$ sowie zwischen $\mathrm{t} 1$ und $\mathrm{t} 3$. Die geringe Mittelwertsenkung von $\mathrm{t} 2 \mathrm{zu} \mathrm{t} 3$ ist nicht signifikant (vgl. Tabelle 23).

Tabelle 23: Paarvergleich Sauerstoffaufnahme (Spiroergometrie).

\begin{tabular}{|l|l|r|r|r|}
\hline \hline \multicolumn{7}{|c|}{ Test bei gepaarten Stichproben } \\
\hline & \multicolumn{2}{|c|}{ Gepaarte Differenzen } & \\
\cline { 3 - 5 } & Mittelwert & Standardabweichung & Sig. (2-seitig) \\
\hline Paaren 1 & $\begin{array}{l}\text { Sauerstoffaufnahme bei Belastung in t1 } \\
\text { Sauerstoffaufnahme bei Belastung in t2 }\end{array}$ &, 27696 &, 38398 &, 018 \\
\hline Paaren 2 & $\begin{array}{l}\text { Sauerstoffaufnahme bei Belastung in t1 } \\
\text { Sauerstoffaufnahme bei Belastung in t3 }\end{array}$ &, 40678 &, 38084 &, 002 \\
\hline Paaren 3 & $\begin{array}{l}\text { Sauerstoffaufnahme bei Belastung in t2 } \\
\text { Sauerstoffaufnahme bei Belastung in t3 }\end{array}$ &, 12982 &, 31084 &, 142 \\
\hline \hline
\end{tabular}

Auch im respiratorischen Quotienten (vgl. Tabelle 24) lassen sich tendenziell signifikante Unterschiede erkennen. Zwischen $\mathrm{t} 1$ und $\mathrm{t} 2$ zeigt sich ein signifikanter Unterschied und zwischen $\mathrm{t} 1$ und $\mathrm{t} 3$ zumindest eine tendenzielle $(\mathrm{p}<0,1)$ Signifikanz. Dagegen gibt es mutmaßlich keinen Unterschied zwischen $\mathrm{t} 2$ und $\mathrm{t} 3$.

Tabelle 24: Paarvergleich RQ (Spiroergometrie).

\begin{tabular}{|l|l|r|r|r|}
\hline \hline \multicolumn{7}{|c|}{ Test bei gepaarten Stichproben } \\
\hline & \multicolumn{2}{|c|}{ Gepaarte Differenzen } & \multirow{2}{*}{ Sig. (2-seitig) } \\
\cline { 3 - 4 } & & Mittelwert & Standardabweichung &, 010 \\
\hline Paaren 1 & RQ in t1 - RQ in t2 &,- 06891 &, 08557 &, 074 \\
\hline Paaren 2 & RQ in t1 - RQ in t3 &,- 04790 &, 09227 &, 384 \\
\hline Paaren 3 & RQ in t2 - RQ in t3 &, 02100 &, 08730 & \\
\hline \hline
\end{tabular}

Beim dritten Paarvergleich bezogen auf den Energieumsatz (vgl. Tabelle 25) zeigen sich ebenfalls signifikante Differenzen zwischen $\mathrm{t} 1$ und $\mathrm{t} 2$, sowie zwischen $\mathrm{t} 1$ und $\mathrm{t} 3$. Der relative Energieumsatz in $\mathrm{t} 1$ und $\mathrm{t} 2$ differiert tendenziell signifikant.

Tabelle 25: Paarvergleich relativer Energieumsatz (Spiroergometrie).

\begin{tabular}{|l|l|r|r|r|}
\hline \hline \multicolumn{7}{|c|}{ Test bei gepaarten Stichproben } \\
\hline & \multicolumn{2}{|c|}{ Gepaarte Differenzen } & \multirow{2}{*}{ Sig. (2-seitig) } \\
\cline { 3 - 5 } & Mittelwert & Standardabweichung &, 013 \\
\hline Paaren 1 & $\begin{array}{l}\text { Relativer Energieumsatz in t1 } \\
\text { Relativer Energieumsatz in t2 }\end{array}$ &, 01499 &, 01946 &, 001 \\
\hline Paaren 2 & $\begin{array}{l}\text { Relativer Energieumsatz in t1 } \\
\text { Relativer Energieumsatz in t3 }\end{array}$ &, 02262 &, 02056 &, 116 \\
\hline Paaren 3 & $\begin{array}{l}\text { Relativer Energieumsatz in t2 } \\
\text { Relativer Energieumsatz in t3 }\end{array}$ &, 00762 &, 01695 & \\
\hline \hline
\end{tabular}


Die dargestellten Werte beziehen sich auf die einzelnen Messzeitpunkte. Da die Forstwirte unterschiedliche Tätigkeiten in den Messzeitpunkten durchgeführt haben, werden in Tabelle 26 die Energieumsätze unabhängig von den Messzeitpunkten nur in Bezug auf die ausgeführten Tätigkeiten aufgeführt.

Tabelle 26: Zuordnung von Energieumsatz und Tätigkeiten der Forstwirte.

\begin{tabular}{|l|r|r|r|r|}
\hline \hline & $\begin{array}{c}\text { Anzahl der } \\
\text { Untersuchungen }\end{array}$ & EU in kcal/ min & $\begin{array}{c}\text { rel EU in kcal/ kg } \\
\mathrm{KG} / \mathrm{min}\end{array}$ & $\mathrm{VO}_{2}$ in $\mathrm{l} / \mathrm{min}$ \\
\hline Holzernte & 19 & 9,40 & 0,110 & 1,94 \\
\hline Läuterung & 9 & 8,93 & 0,103 & 1,82 \\
\hline Pflanzung & 8 & 8,24 & 0,094 & 1,68 \\
\hline Sammelhieb & 4 & 8,07 & 0,093 & 1,64 \\
\hline Wegebau & 2 & 6,67 & 0,069 & 1,35 \\
\hline \hline
\end{tabular}

Aus der Tabelle lässt sich ablesen, dass in den drei Messzeitpunkten die Holzernte am Häufigsten von den Forstwirten ausgeführt wurde. Bei dieser Tätigkeit findet der höchste Energieumsatz mit durchschnittlich 9,4 kcal/ min bzw. einer Sauerstoffaufnahme von 1,94 1/ min statt. Die beiden weiteren motormanuellen Tätigkeiten liegen mit 8,93 kcal/ min bei der Läuterung und 8,07 kcal/ min beim Sammelhieb unter dem Energieumsatz bei der Holzernte. Bei Pflanzungen weisen die Probanden einen Energieumsatz von 8,24 kcal/ min und beim Wegebau einen Energieumsatz von $6,67 \mathrm{kcal} / \mathrm{min}$ auf.

Um eine Beurteilung der Energieumsätze zu erlauben, werden in Tabelle 27 der Grundumsatz und die Tätigkeiten dargestellt sowie deren Energieumsatz in kcal/ min und deren entsprechendes MET zugeordnet.

Tabelle 27: MET der einzelnen gemessenen Tätigkeiten.

\begin{tabular}{|l|r|r|}
\hline \hline \multicolumn{1}{|c|}{ Situation } & \multicolumn{1}{|c|}{$\begin{array}{c}\text { Energieumsatz } \\
\text { in kcal/ min }\end{array}$} & \multicolumn{1}{c|}{ MET } \\
\hline Grundumsatz & 1,26 & 1 \\
\hline Holzernte & 9,4 & 7,46 \\
\hline Läuterung & 8,9 & 7,06 \\
\hline Pflanzung & 8,2 & 6,50 \\
\hline Sammelhieb & 8,0 & 6,34 \\
\hline Wegebau & 6,6 & 5,23 \\
\hline \hline
\end{tabular}

Aus der Tabelle lässt sich schließen, dass die Tätigkeiten der Forstwirte mit einem MET zwischen fünf und sieben definiert werden können.

Eine Zuordnung der erhobenen Werte in die Tabelle bisher ermittelter Daten findet in Tabelle 28 statt. Es ist festzustellen, dass die Werte mit 6,6-9,4 kcal/ min recht hoch sind. Allerdings liegen Werte bei unterschiedlichen Tätigkeiten vor. Lediglich bei Holzernte ist ein direkter Vergleich möglich. Die von Fleischer (2009) zitierten Werte liegen mit 5,2-5,5 kcal/ min unter dem Wert der vorliegenden Studie mit 9,4 kcal/ min. 
Tabelle 28: Arbeitsenergieumsatz in kcal/ min bei Forstwirten.

\begin{tabular}{|l|r|r|r|r|r|r|}
\hline \hline \multicolumn{1}{|c|}{ Tätigkeit } & $\begin{array}{c}\text { Vorliegende } \\
\text { Studie }\end{array}$ & $\begin{array}{c}\text { Spitzer- } \\
\text { Hettinger } \\
(1969)\end{array}$ & $\begin{array}{c}\text { Valentin } \\
(1979)\end{array}$ & $\begin{array}{c}\text { Rost } \\
(2001)\end{array}$ & $\begin{array}{c}\text { FAO/ } \\
\text { WHO/ } \\
\text { UNU } \\
(2001)\end{array}$ & $\begin{array}{c}\text { Kaminsky (1960) \& } \\
\text { Stentzel (1957) (zi- } \\
\text { tiert in Fleischer } \\
\text { 2009) }\end{array}$ \\
\hline $\begin{array}{l}\text { Gehen (ohne Last } \\
\text { in der Ebene) }\end{array}$ & & $2,1-5,0$ & 4,3 & 3,1 & & $3,2-4,3$ \\
\hline $\begin{array}{l}\text { Steigen (ohne Last } \\
\text { am Steilhang) }\end{array}$ & & $7,2-14,4$ & & & & \\
\hline $\begin{array}{l}\text { Bergabgehen } \\
\text { (ohne Last am } \\
\text { Steilhang) }\end{array}$ & & $2,8-9,4$ & & & & \\
\hline $\begin{array}{l}\text { Pflanzen in vorbe- } \\
\text { reiteten Löchern }\end{array}$ & & $2,2-3,3$ & & & & \\
\hline Pflanzen & 8,2 & & & & & 5,3 \\
\hline $\begin{array}{l}\text { Holzernte (Reine } \\
\text { AZ) }\end{array}$ & 9,4 & & & & $5,4-8,0$ & \\
\hline $\begin{array}{l}\text { Fällschnitt mit } \\
\text { EMS }\end{array}$ & & $4,3-6,5$ & & & & 5,7 \\
\hline Vermessen & 8,9 & & & & & \\
\hline Läuterung & 6,0 & & & & & \\
\hline Sammelhieb & 6,6 & & & & & \\
\hline Wegebau & & & & & \\
\hline \hline
\end{tabular}

Im Folgenden werden die Messung des Ruheenergieumsatzes und dessen Berechnung mit dem KÄ und die Grundumsätze, die mittels der Formel nach Mifflin und mit dem KÄ errechnet wurden, gegenübergestellt und miteinander verglichen. Zu berücksichtigen ist, dass aufgrund der arbeitsbedingten Umstände bei der spiroergometrischen Untersuchung der $\mathrm{Ru}$ heenergieumsatz nicht unter standardisierten Bedingungen gemessen werden konnte.

Tabelle 29: Gemessene RMR und errechnete RMR und BMR (in kcal/ min).

\begin{tabular}{|c|r|r|r|r|}
\hline \hline & $\begin{array}{c}\text { RMR bei } \\
\text { Spiroergometrie }\end{array}$ & BMR nach Mifflin & RMR nach KÄ & BMR nach KÄ \\
\hline $\mathrm{t} 1(87 \mathrm{~kg})$ & 2,30 & 1,25 & 1,55 & 1,41 \\
\hline $\mathrm{t} 2(88 \mathrm{~kg})$ & 2,04 & 1,26 & 1,54 & 1,40 \\
\hline $\mathrm{t} 3(89 \mathrm{~kg})$ & 2,12 & 1,27 & 1,56 & 1,42 \\
\hline $\mathrm{MW}(88 \mathrm{~kg})$ & 2,15 & 1,26 & 1,55 & 1,41 \\
\hline \hline
\end{tabular}

Laut Literatur ist bei den Probanden mit einem durchschnittlichen Gewicht von $88 \mathrm{~kg}$ ein Grundumsatz mit einem $\mathrm{VO}_{2}$-Wert von $308 \mathrm{ml} / \mathrm{min}$ bzw. einem Kalorienverbrauch von 1,4 kcal/ min zu erwarten. Der Ruheumsatz liegt mit 338,8 ml/ min und 1,5 kcal/ min entsprechend $10 \%$ über dem Wert des Grundumsatzes (vgl. Kapitel 3).

Der Durchschnitt der errechneten Werte nach Mifflin für den Grundumsatz liegt bei $1,26 \mathrm{kcal} / \mathrm{min}$. Der entsprechende Wert, der mit dem $\mathrm{K} \ddot{\mathrm{A}}$ errechnet wurde, liegt bei 1,4 kcal/ min. Eine noch größere Diskrepanz liegt bei den gemessenen und errechneten Ruheenergieumsätzen vor. Mit dem KÄ wurde ein Wert von 1,5 kcal/ min errechnet, der dem spiroergometrisch gemessenen Wert vor der beruflichen Tätigkeit von 2,1 kcal/ min gegen- 
über steht. Diese Diskrepanzen zwischen den einzelnen Werten wiederholen sich in den einzelnen Messzeitpunkten. Es ergeben sich somit gemessene Werte, die über dem zu erwartenden Ruheumsatz liegen.

\subsubsection{Fahrradspiroergometrie}

An der fahrradergometrischen Untersuchung nahmen aus organisatorischen und finanziellen Gründen lediglich FW01, FW03, FW04 und FW05 teil. Die genannten Forstwirte führten alle in $\mathrm{t} 1$ motormanuelle Holzernte, in $\mathrm{t} 2$ Pflanzungen (Rhodener Pflanzverfahren) und in $\mathrm{t} 3 \mathrm{Jung}$ bestandspflege durch.

Um die erhobenen Werte während der beruflichen Tätigkeit in Watt ausdrücken zu können, wurden passend zu den individuellen Werten des jeweiligen Messzeitpunktes Leistungen in Watt geschätzt, um bei der Fahrradspiroergometrie ähnliche Werte wie bei der beruflichen Tätigkeit zu erlangen. Tabelle 30 gibt die Leistungen in Watt wider, die von den Forstwirten zu den drei Messzeitpunkten absolviert wurden.

Tabelle 30: Leistungen in Watt der Forstwirte bei der Fahrradspiroergometrie.

\begin{tabular}{|l|r|r|r|}
\hline \hline & Leistung 1 (in W) & Leistung 2 (in W) & \multicolumn{1}{|c|}{ Leistung 3 (in W) } \\
\hline FW01 & 100 & 140 & 180 \\
\hline FW03 & 100 & 140 & 160 \\
\hline FW04 & 100 & 130 & 150 \\
\hline FW05 & 100 & 90 & 120 \\
\hline \hline
\end{tabular}

Die Leistungen in Watt wurden in Anpassung an die berufliche Tätigkeit geschätzt (vgl. Kapitel 6.3.5.2). Auffällig ist zum einen, dass FW05 einen deutlich geringeren Energieumsatz aufweist, so dass hier eine Wattstufe unter $100 \mathrm{~W}$ gewählt wurde. Zum anderen weist FW01 eine deutlich höhere Leistung in Watt im Vergleich zu den anderen drei Forstwirten auf. Mit einer höchsten Leistung von $180 \mathrm{~W}$ liegt dieser Wert $20 \mathrm{~W}, 30 \mathrm{~W}$ bzw. $60 \mathrm{~W}$ über den höchsten Leistungen der anderen Probanden.

Zunächst werden die Messdaten des relativen Energieumsatzes in den unterschiedlichen Messzeitpunkten und bei den unterschiedlichen Wattstufen gegenübergestellt. Es folgt in Tabelle 31 eine Einzelauflistung der Ergebnisse der Forstwirte sowie eine Darstellung des Mittelwertes der Energieumsätze bei den einzelnen Messungen. 
Tabelle 31: Relativer Energieumsatz bei Fahrradspiroergometrie in kcal/ kg KG/ min.

\begin{tabular}{|c|c|c|c|r|r|r|r|r|r|}
\hline \hline \multirow{2}{*}{} & \multicolumn{3}{|c|}{ rel. EU in t1 } & \multicolumn{3}{c|}{ rel. EU in t2 } & \multicolumn{3}{c|}{ rel. EU int3 } \\
\cline { 2 - 11 } & W1 & W2 & \multicolumn{1}{c|}{ W3 } & W1 & W2 & W3 & W1 & W2 & W3 \\
\hline FW01 &, 077 &, 095 &, 116 &, 103 &, 126 &, 161 &, 094 &, 119 &, 149 \\
\hline FW03 &, 093 &, 122 &, 132 &, 100 &, 128 &, 142 &, 091 &, 119 &, 133 \\
\hline FW04 &, 089 &, 109 &, 126 &, 084 &, 103 &, 116 &, 091 &, 111 &, 129 \\
\hline FW05 &, 086 &, 079 &, 101 &, 083 &, 077 &, 097 &, 078 &, 070 &, 090 \\
\hline MW &, 086 &, 101 &, 118 &, 092 &, 108 &, 129 &, 089 &, 105 &, 125 \\
\hline \hline
\end{tabular}

Der Tabelle ist zu entnehmen, dass die Mittelwerte in $\mathrm{t} 1$ die niedrigsten relativen Energieumsätze wiedergeben. In t2 werden die höchsten Mittelwerte bzgl. des relativen Energieumsatzes erreicht. Es ist jedoch zu berücksichtigen, dass lediglich bei W1 eine identische Leistung in Watt von $100 \mathrm{~W}$ von allen drei Forstwirten bewältigt wird. Der Mittelwert der relativen Energieumsätze beträgt in $\mathrm{t} 10,086 \mathrm{kcal} / \mathrm{min} / \mathrm{kg}$, in $\mathrm{t} 20,092 \mathrm{kcal} / \mathrm{min} / \mathrm{kg}$ und in $\mathrm{t} 30,089 \mathrm{kcal} /$ min/ kg. Eine Betrachtung der relativen Energieumsätze der einzelnen Forstwirte erfolgt nachstehend mit Hilfe grafischer Darstellungen (vgl. Abbildungen 21-24).

Für eine vollständige Betrachtung der zugeordneten Leistungsbereiche in Watt werden in Tabelle 32 die anhand des relativen Energieumsatzes und der Sauerstoffaufnahme ermittelten Leistungsbereiche in Watt gegenübergestellt.

Tabelle 32: Zugeordnete Leistung in Watt zum relativen Energieumsatz und zur Sauerstoffaufnahme.

\begin{tabular}{|l|r|r|r|r|r|r|}
\hline \hline & \multicolumn{2}{|c|}{$\mathrm{t} 1$} & \multicolumn{2}{c|}{$\mathrm{t} 2$} & \multicolumn{2}{c|}{$\mathrm{t} 3$} \\
\cline { 2 - 7 } & Rel. EU & \multicolumn{1}{|c|}{$\mathrm{VO}_{2}$} & Rel. EU & $\mathrm{VO}_{2}$ & Rel. EU & $\mathrm{VO}_{2}$ \\
\hline FW01 & 200 & 190 & 140 & 140 & 140 & 140 \\
\hline FW03 & 160 & 160 & 130 & 140 & 120 & 130 \\
\hline FW04 & 140 & 140 & 130 & 140 & 130 & 120 \\
\hline FW05 & 110 & 110 & 120 & 120 & 110 & 115 \\
\hline \hline
\end{tabular}

Generell kann festgestellt werden, dass ein höherer Energieumsatz und eine höhere Sauerstoffaufnahme einen höheren Leistungsbereich in Watt bedingen. Die Zuordnung mittels der beiden Parameter ergibt annähernd dieselben Leistungsbereiche in Watt, so dass den im Feld gemessenen Tätigkeiten in $\mathrm{t} 1$ eine Leistung von 110 bis $190 \mathrm{~W}$, in t2 eine Leistung von 120 bis $140 \mathrm{~W}$ und in $\mathrm{t} 3$ eine Leistung von 115 bis $140 \mathrm{~W}$ zugeschrieben werden kann. Auffällig ist auch in dieser Darstellung, dass es interpersonelle Varianzen gibt. Darüber hinaus lässt sich feststellen, dass FW04 und FW05 in allen drei Messzeitpunkten wenig intrapersonelle 
Varianzen aufweisen, wohingegen FW01 und FW03 in t1 eine deutlich höhere Leistung als in $\mathrm{t} 2$ und $\mathrm{t} 3$ erbringen.

In Abbildung 21 wird der relative Energieumsatz der Messung bei beruflicher Tätigkeit (direkt auf der y-Achse eingezeichnet) sowie bei der Fahrradspiroergometrie von FW01 in den drei Messzeitpunkten dargestellt. In t1 ist der relative Energieumsatz mit einem Wert von $0,14 \mathrm{kcal} / \mathrm{min} / \mathrm{kg}$ bei der beruflichen Tätigkeit am höchsten. Der Energieumsatz ist in $\mathrm{t} 2$ und t3 mit einem Wert von $0,12 \mathrm{kcal} / \mathrm{min} / \mathrm{kg}$ nahezu identisch. Der relative Energieumsatz in den einzelnen Wattstufen bei der Fahrradspiroergometrie nimmt gleichmäßig zu, wobei in $\mathrm{t} 1$ die geringsten Werte $(0,08 / 0,1 / 0,12 \mathrm{kcal} / \mathrm{min} / \mathrm{kg})$ und in $\mathrm{t} 2$ die höchsten Werte $(0,1 / 0,12 / 0,16$ $\mathrm{kcal} / \mathrm{min} / \mathrm{kg}$ ) erreicht werden. Setzt man nun die Werte in Relation, so lässt sich der relative Energieumsatz für die berufliche Tätigkeit in $\mathrm{t} 1$ mit über $180 \mathrm{~W}$ gleichsetzen. Der relative Energieumsatz in $\mathrm{t} 2$ und $\mathrm{t} 3$ fällt ungefähr auf $140 \mathrm{~W}$.

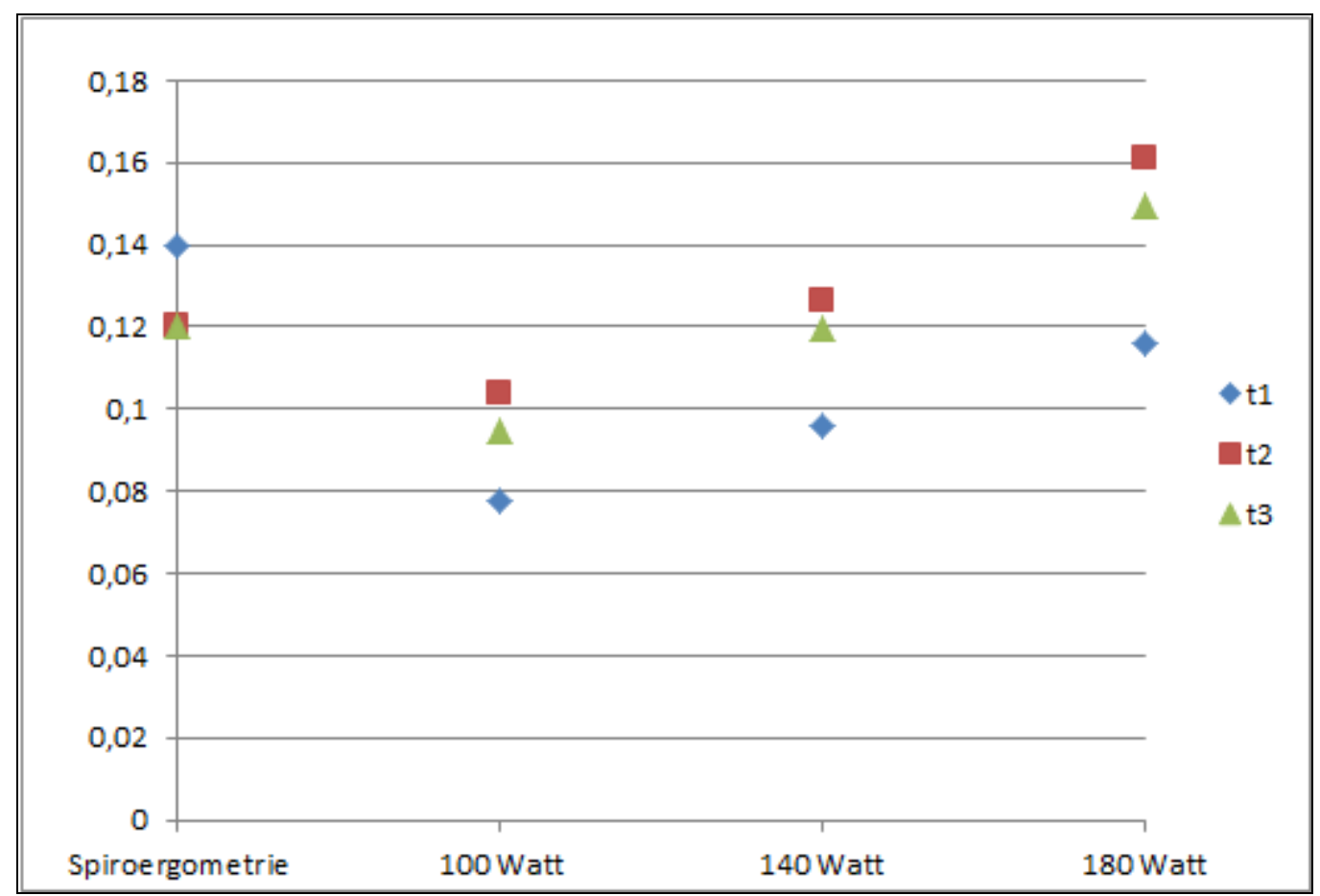

Abbildung 21: Relativer Energieumsatz FW01 bei Spiroergometrie und Fahrradspiroergometrie (FE). 
Der relative Energieumsatz von FW03 wird in Abbildung 22 visualisiert. Beträgt der Energieumsatz in $\mathrm{t} 1$ bei beruflicher Tätigkeit $0,14 \mathrm{kcal} / \mathrm{min} / \mathrm{kg}$, so entspricht dieser Wert in etwa dem Energieumsatz, der in t1 während der Fahrradspiroergometrie bei $160 \mathrm{~W}$ erreicht wird. Der Energieumsatz in $\mathrm{t} 2$ von $0,12 \mathrm{kcal} / \mathrm{min} / \mathrm{kg}$ ist in etwa dem Energieumsatz gleichzusetzen, der bei $130 \mathrm{~W}$ entsteht. In $\mathrm{t} 3$ entspricht der relative Energieumsatz bei beruflicher Tätigkeit mit einem Wert von $0,11 \mathrm{kcal} / \mathrm{min} / \mathrm{kg}$ in etwa $120 \mathrm{~W}$.

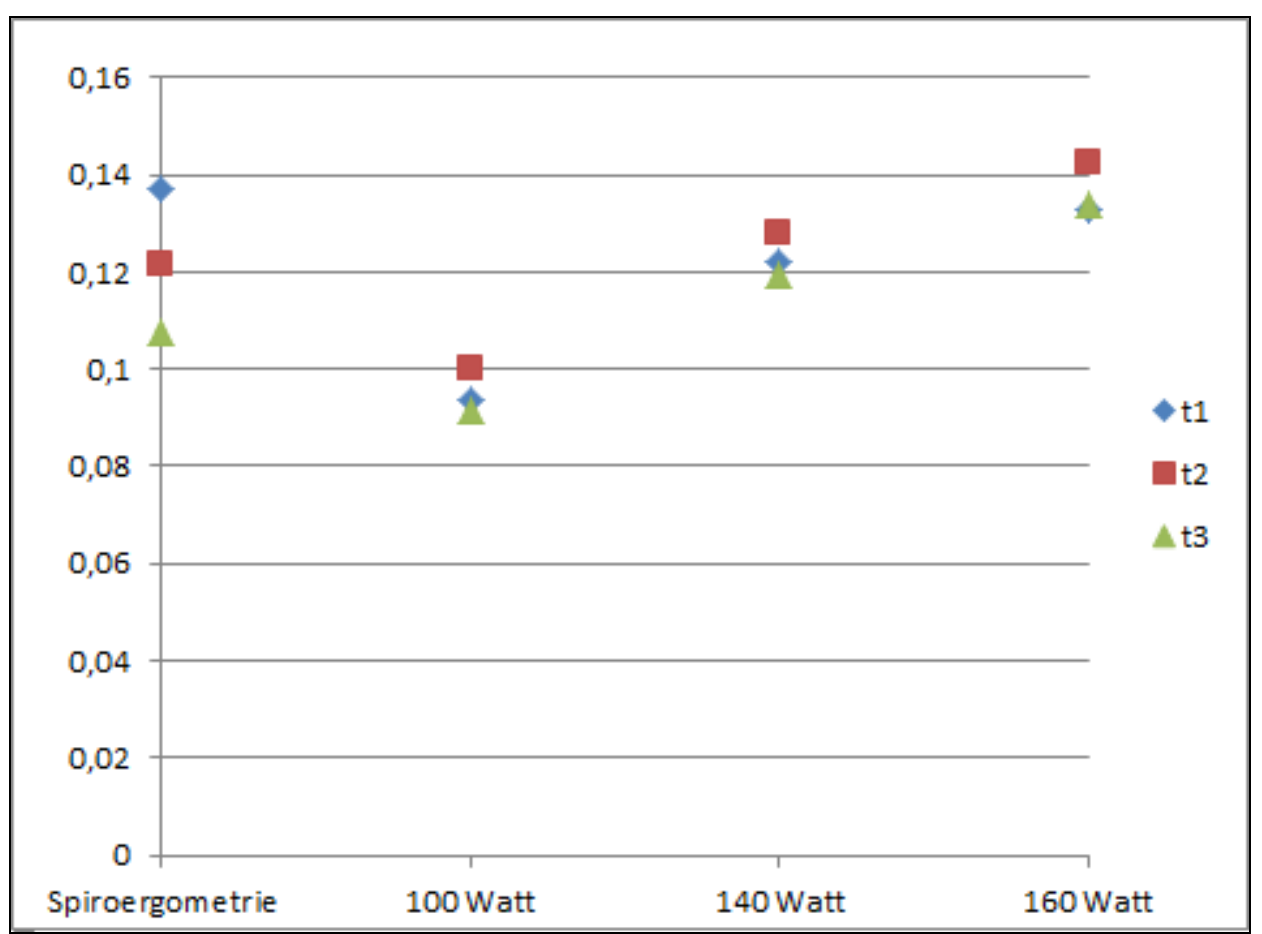

Abbildung 22: Relativer Energieumsatz FW03 bei Spiroergometrie und Fahrradspiroergometrie (FE). 
In der folgenden Abbildung 23 wird der relative Energieumsatz bei FW04 dargestellt. Der relative Energieumsatz in $\mathrm{t} 1(0,12 \mathrm{kcal} / \mathrm{min} / \mathrm{kg})$ bei beruflicher Tätigkeit ist einem Wert von ca. $140 \mathrm{~W}$ gleichzusetzen. In t2 und t3 wird ein relativer Energieumsatz von ca. $0,11 \mathrm{kcal} / \mathrm{min} / \mathrm{kg}$ erreicht, welcher in beiden Messzeitpunkten mit dem relativen Energieumsatz bei $130 \mathrm{~W}$ korreliert.

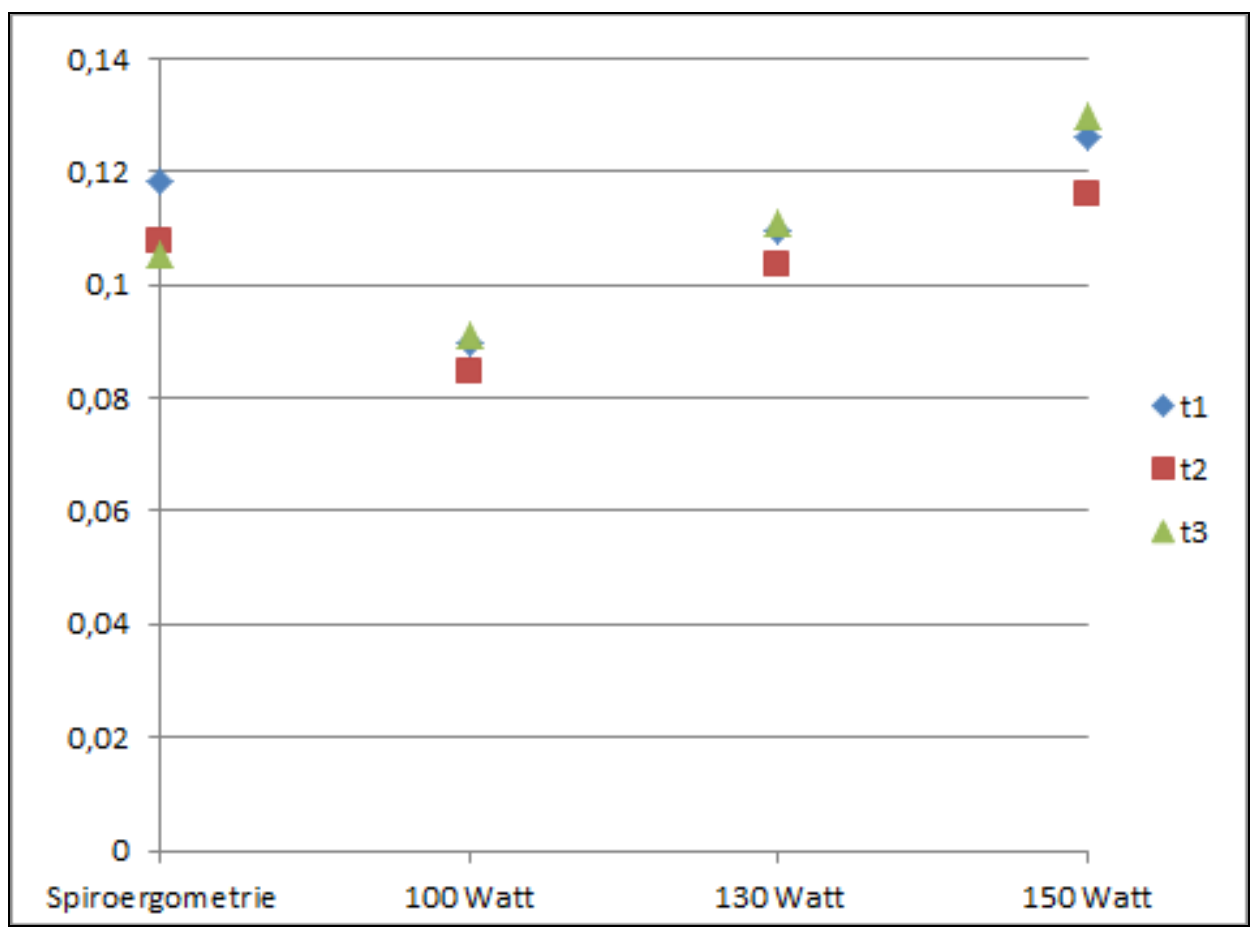

Abbildung 23: Relativer Energieumsatz FW04 bei Spiroergometrie und Fahrradspiroergometrie (FE). 
Abbildung 24 gibt die Werte des relativen Energieumsatzes von FW05 wider. Der relative Energieumsatz von FW05 beträgt sowohl in t1 als auch in $\mathrm{t} 3$ bei beruflicher Tätigkeit $0,09 \mathrm{kcal} / \mathrm{min} / \mathrm{kg}$, welcher bei einer Wattstufe in beiden Messzeitpunkten von ca. $110 \mathrm{~W}$ erreicht wird. In t2 kann der relative Energieumsatz von $0,1 \mathrm{kcal} / \mathrm{min} / \mathrm{kg}$ mit dem Energieumsatz der Fahrradspiroergometrie bei $120 \mathrm{~W}$ in Relation gesetzt werden.

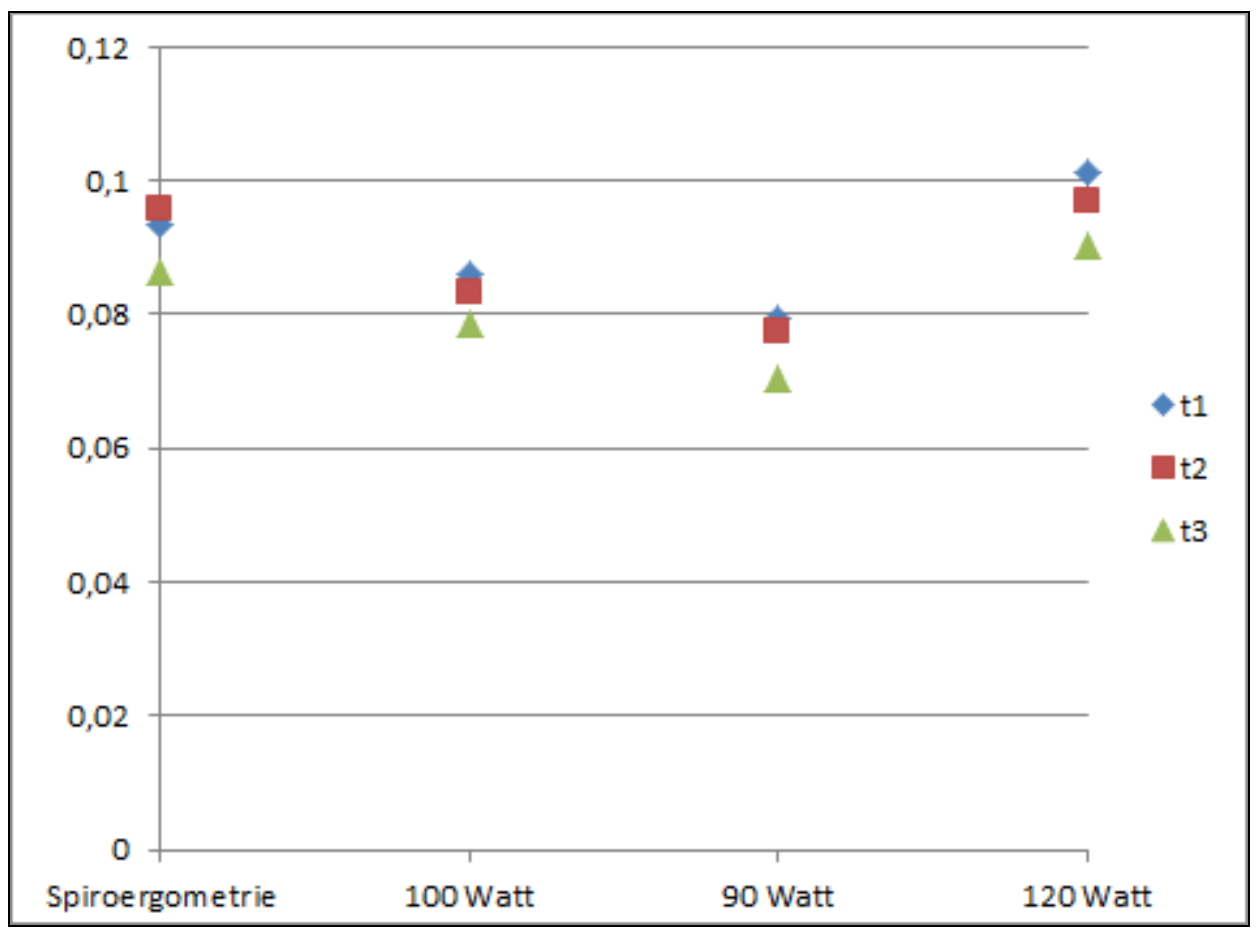

Abbildung 24: Relativer Energieumsatz FW05 bei Spiroergometrie und Fahrradspiroergometrie (FE).

In Tabelle 33 werden die relativen Energieumsätze der Forstwirte den entsprechenden Leistungen in Watt gegenübergestellt.

Tabelle 33: Zuordnung des relativen Energieumsatzes (in kcal/ kg KG/ min) bei beruflicher Tätigkeit und der korrelierenden Leistung in Watt.

\begin{tabular}{|c|c|c|c|c|c|c|}
\hline & \multicolumn{2}{|l|}{$\mathrm{t} 1$} & \multicolumn{2}{|l|}{$\mathrm{t} 2$} & \multicolumn{2}{|l|}{$\mathrm{t} 3$} \\
\hline & rel. EU & $\mathrm{W}$ & rel. EU & $\mathrm{W}$ & rel. EU & $\mathrm{W}$ \\
\hline FW01 & 0,14 & 200 & 0,12 & 140 & 0,12 & 140 \\
\hline FW03 & 0,14 & 160 & 0,12 & 130 & 0,11 & 120 \\
\hline FW04 & 0,12 & 140 & 0,11 & 130 & 0,11 & 130 \\
\hline FW04 & 0,09 & 110 & 0,1 & 120 & 0,09 & 110 \\
\hline
\end{tabular}

Es lässt sich konstatieren, dass zum einen die relativen Energieumsätze der einzelnen Forstwirte unterschiedlichen Leistungen in Watt bei der Fahrradspiroergometrie entsprechen. Zum anderen erreichen die Forstwirte bei den beruflichen Tätigkeiten in zwei Messzeitpunkten z. T. ähnlich hohe relative Energieumsätze, die dann auch in beiden Messzeitpunkten der gleichen Leistung in Watt entsprechen. 
Im nachfolgenden Abschnitt wird die Sauerstoffaufnahme während der beruflichen Tätigkeit im Vergleich zur Sauerstoffaufnahme bei der Fahrradspiroergometrie thematisiert. Zunächst bildet eine deskriptive Statistik die Werte der Sauerstoffaufnahme in allen drei Messzeitpunkten zu den drei Wattstufen ab.

Tabelle 34: Sauerstoffaufnahme bei Fahrradspiroergometrie.

\begin{tabular}{|c|c|c|c|c|c|c|c|c|c|}
\hline \hline & \multicolumn{3}{|c|}{$\mathrm{VO}_{2}$ (in 1/ min) in $\mathrm{t} 1$} & \multicolumn{2}{c|}{$\mathrm{VO}_{2}$ (in 1/ min) in $\mathrm{t} 2$} & \multicolumn{3}{c|}{$\mathrm{VO}_{2}$ (in 1/ min) in $\mathrm{t} 3$} \\
\cline { 2 - 10 } & $\mathrm{W} 1$ & $\mathrm{~W} 2$ & $\mathrm{~W} 3$ & $\mathrm{~W} 1$ & $\mathrm{~W} 2$ & $\mathrm{~W} 3$ & $\mathrm{~W} 1$ & $\mathrm{~W} 2$ & $\mathrm{~W} 3$ \\
\hline FW01 & 1,32 & 1,62 & 1,95 & 1,61 & 1,95 & 2,48 & 1,53 & 1,91 & 2,36 \\
\hline FW03 & 1,54 & 2,00 & 2,18 & 1,60 & 2,05 & 2,28 & 1,48 & 1,90 & 2,13 \\
\hline FW04 & 1,60 & 1,92 & 2,18 & 1,49 & 1,81 & 2,01 & 1,54 & 1,86 & 2,15 \\
\hline FW05 & 1,48 & 1,36 & 1,72 & 1,42 & 1,32 & 1,65 & 1,33 & 1,18 & 1,51 \\
\hline MW & 1,485 & 1,725 & 2,0075 & 1,53 & 1,7825 & 2,105 & 1,47 & 1,7125 & 2,0375 \\
\hline \hline
\end{tabular}

In $\mathrm{t} 1$ treten bei FW01 die niedrigsten, in $\mathrm{t} 2$ die höchsten Werte bzgl. der Sauerstoffaufnahme auf. FW03 erzielt in $\mathrm{t} 1$ und $\mathrm{t} 3$ nahezu gleiche Werte der Sauerstoffaufnahme (in $\mathrm{t} 3$ etwas niedriger) und weist in $\mathrm{t} 2$ höhere Werte auf. Bei FW04 treten die höchsten Werte in $\mathrm{t} 3$, die niedrigsten in $\mathrm{t} 2$ auf. FW05 erreicht in $\mathrm{t} 1$ die höchsten und in $\mathrm{t} 3$ die niedrigsten Werte. Betrachtet man die Werte der Forstwirte untereinander, so lässt sich festhalten, dass bei der Leistung von $100 \mathrm{~W}$ (= W1) differierende Werte sowohl zwischen den Forstwirten als auch bei den Forstwirten individuell zu den einzelnen Messzeitpunkten zu erkennen sind. FW05 erreicht somit die niedrigste Sauerstoffaufnahme bei $100 \mathrm{~W}$ mit einem Wert von $15,7 \mathrm{ml} / \mathrm{min} / \mathrm{kg}$ und FW01 die höchste Sauerstoffaufnahme bei $100 \mathrm{~W}$ mit einem Wert von $21,19 \mathrm{ml} / \mathrm{min} / \mathrm{kg}$. 
Eine Betrachtung der Sauerstoffaufnahme der einzelnen Forstwirte erfolgt wiederum anhand grafischer Darstellungen. Die Sauerstoffaufnahme bei beruflicher Tätigkeit von FW01 in t1 entspricht der Sauerstoffaufnahme bei ca. 190 W. In t2 entspricht die Sauerstoffaufnahme einer Leistung von $140 \mathrm{~W}$ ebenso in $\mathrm{t} 3$ (vgl. Abbildung 25).

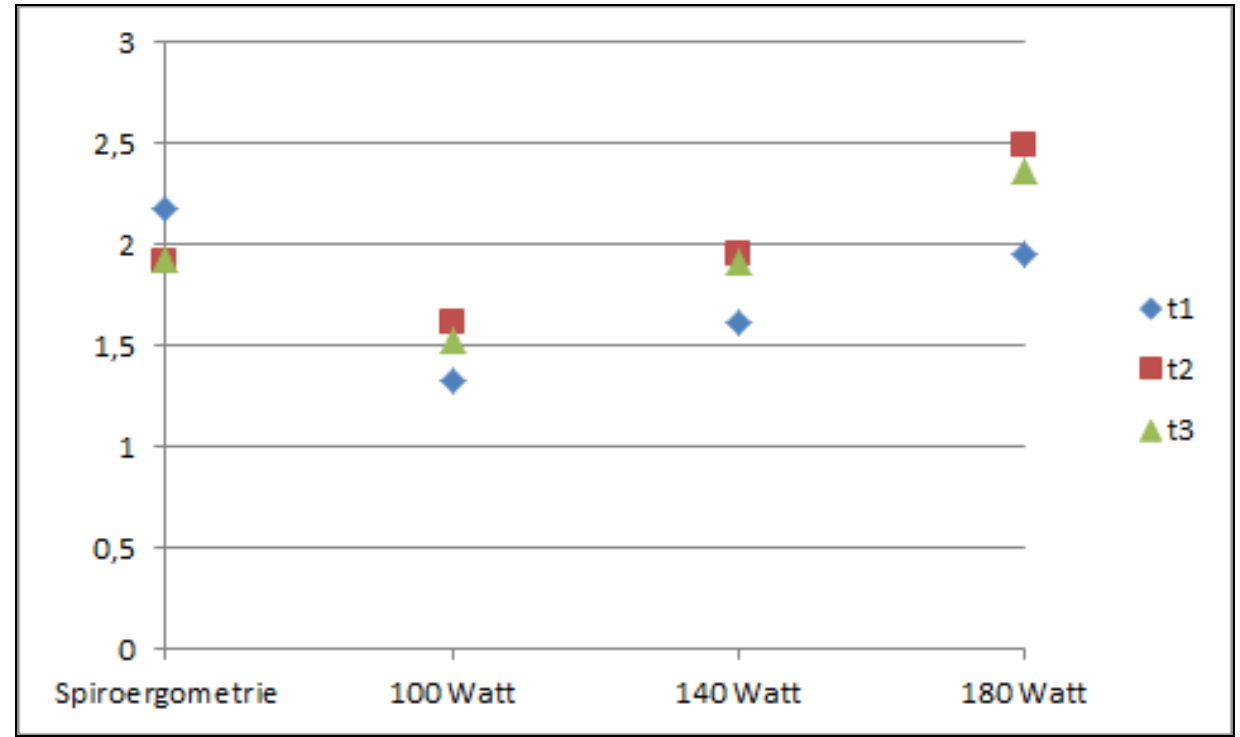

Abbildung 25: Sauerstoffaufnahme FW01 bei Spiroergometrie und Fahrradspiroergometrie (FE).

In Abbildung 26 wird die Sauerstoffaufnahme von FW03 dargestellt. Auch hier lässt sich die Sauerstoffaufnahme bei beruflicher Tätigkeit einer Sauerstoffaufnahme und somit einer Leistung in Watt bei der Fahrradspiroergometrie zuordnen. In t1 entspricht die Sauerstoffaufnahme einer Leistung von $160 \mathrm{~W}$, in t2 einer Leistung von $140 \mathrm{~W}$ und in $\mathrm{t} 3$ einer Leistung von $130 \mathrm{~W}$.

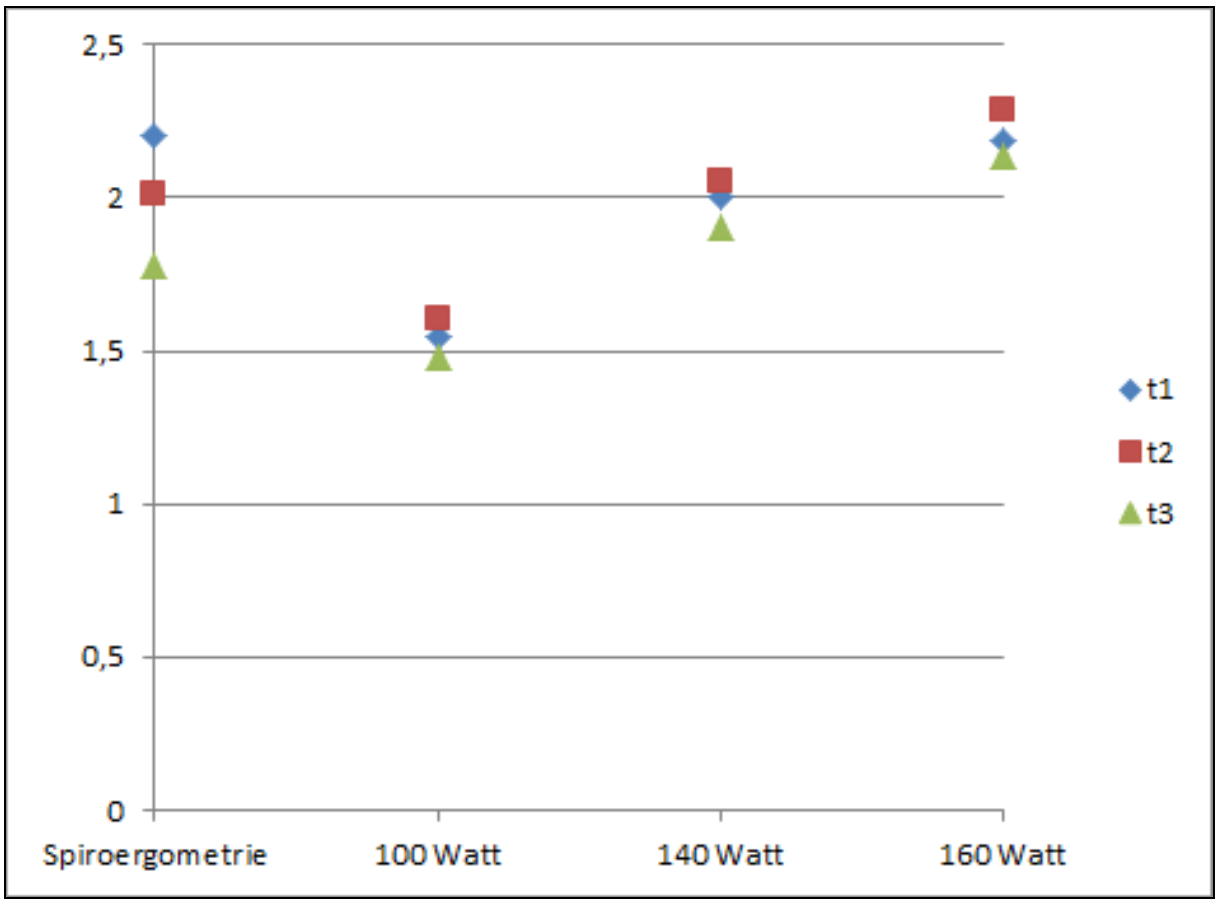

Abbildung 26: Sauerstoffaufnahme FW03 bei Spiroergometrie und Fahrradspiroergometrie (FE). 
Die Sauerstoffaufnahme von FW04 wird in Abbildung 27 visualisiert. In t1 und in t 2 entspricht die Sauerstoffaufnahme bei beruflicher Tätigkeit einer Leistung von $140 \mathrm{~W}$, in t3 einer Leistung von $120 \mathrm{~W}$.

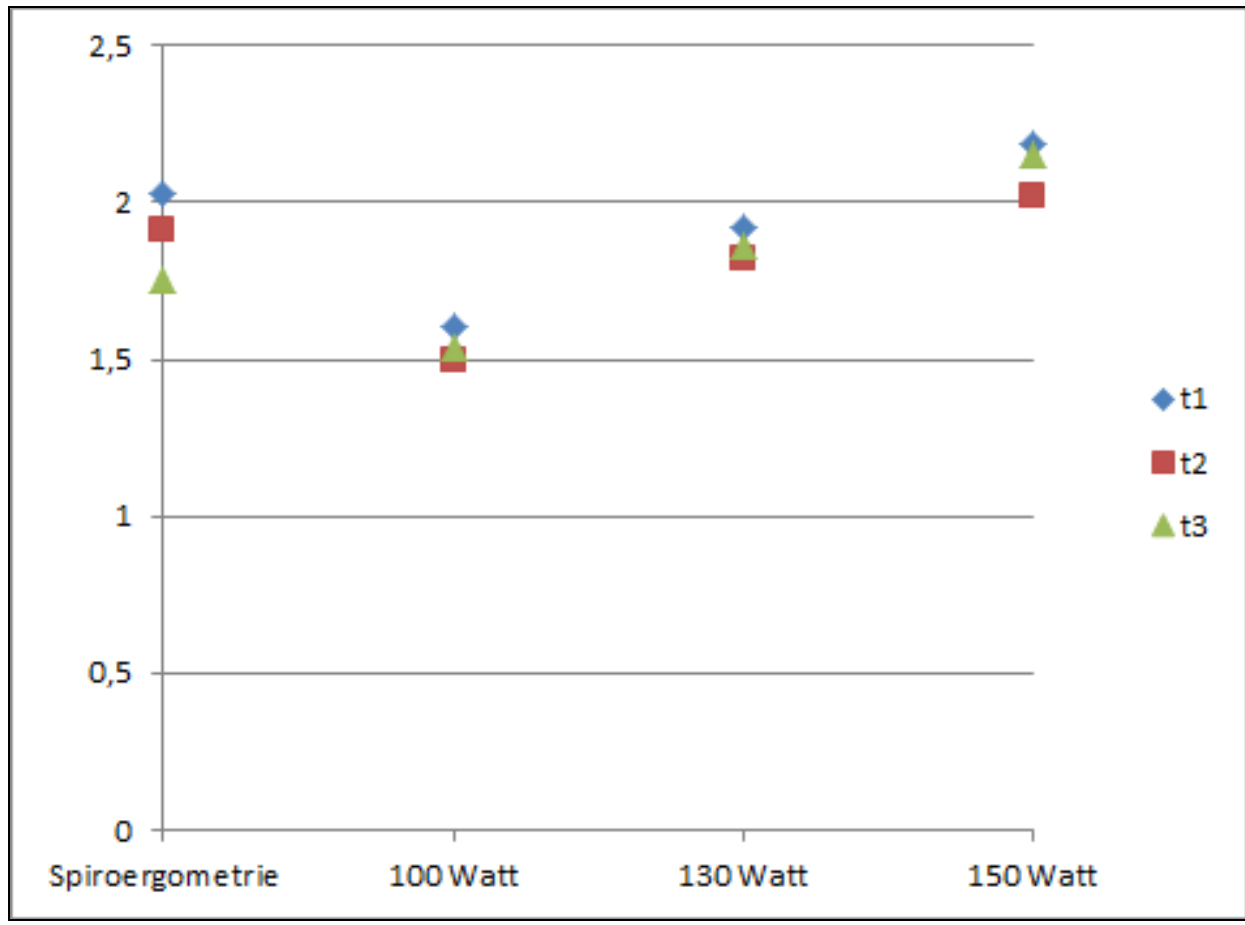

Abbildung 27: Sauerstoffaufnahme FW04 bei Spiroergometrie und Fahrradspiroergometrie (FE).

Die Sauerstoffaufnahme von FW05 entspricht in t1 einer Leistung von $110 \mathrm{~W}$, in t2 einer Leistung von $120 \mathrm{~W}$ und in $\mathrm{t} 2$ einer Leistung von $115 \mathrm{~W}$ (vgl. Abbildung 28).

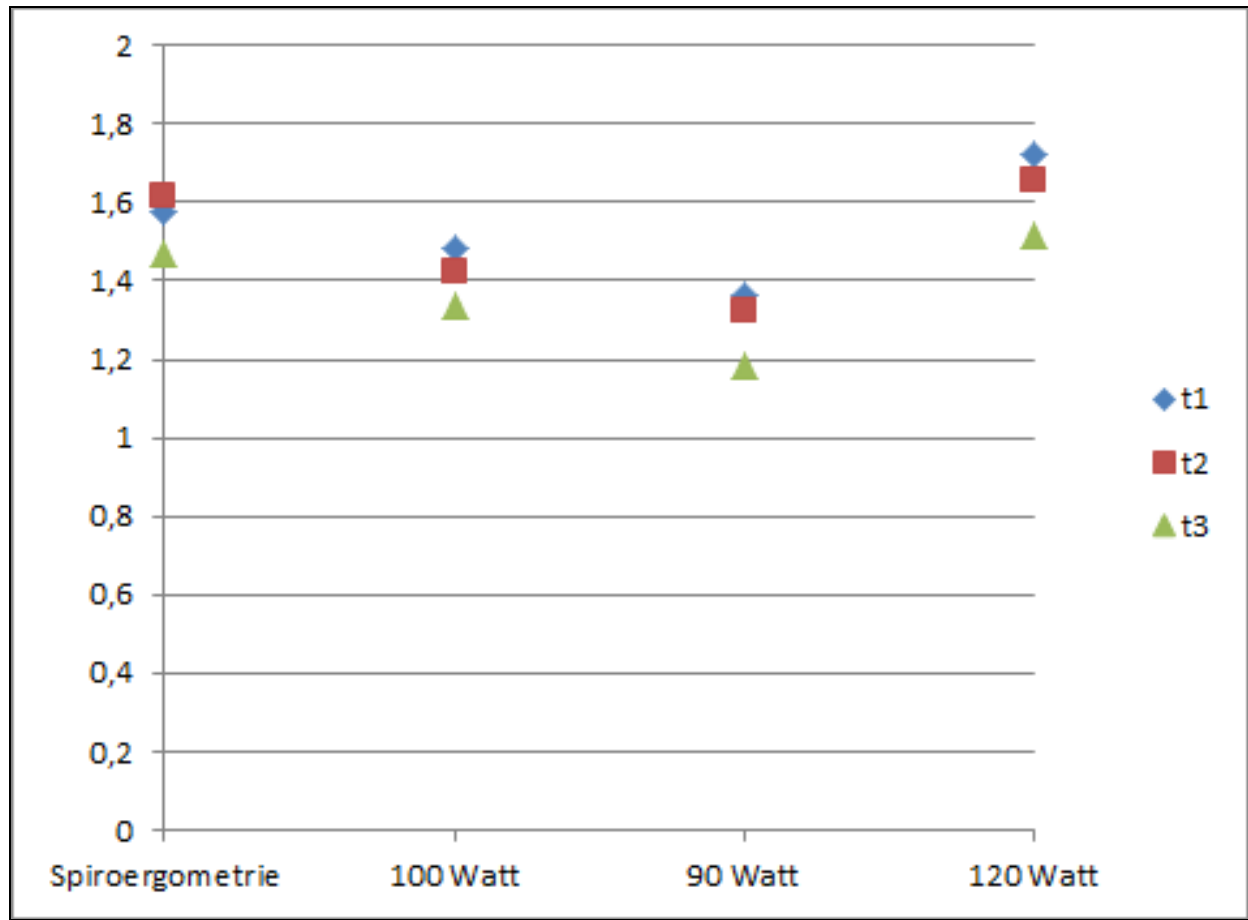

Abbildung 28: Sauerstoffaufnahme FW05 bei Spiroergometrie und Fahrradspiroergometrie (FE). 
Zur Visualisierung werden die Sauerstoffwerte bei beruflicher Tätigkeit in den drei Messzeitpunkten mit den entsprechenden Leistungen in Watt tabellarisch dargestellt (vgl. Tabelle 35).

Tabelle 35: Sauerstoffaufnahme bei beruflicher Tätigkeit und korrelierende Leistungen in Watt.

\begin{tabular}{|c|r|r|r|r|r|r|}
\hline \hline & \multicolumn{2}{|c|}{ in t1 } & \multicolumn{2}{c|}{ in 12} & \multicolumn{2}{c|}{ in $\mathrm{t}$} \\
\cline { 2 - 7 } & $\begin{array}{c}\mathrm{VO}_{2} \\
(1 / \mathrm{min})\end{array}$ & $\mathrm{W}$ & $\begin{array}{c}\mathrm{VO}_{2} \\
(1 / \mathrm{min})\end{array}$ & $\mathrm{W}$ & $\begin{array}{c}\mathrm{VO}_{2} \\
(1 / \mathrm{min})\end{array}$ & $\mathrm{W}$ \\
\hline FW01 & 2,18 & 190 & 1,91 & 140 & 1,92 & 140 \\
\hline FW03 & 2,20 & 160 & 2,01 & 140 & 1,77 & 130 \\
\hline FW04 & 2,02 & 140 & 1,91 & 140 & 1,75 & 120 \\
\hline FW05 & 1,57 & 110 & 1,61 & 120 & 1,46 & 115 \\
\hline \hline
\end{tabular}

Ähnlich wie bei der Betrachtung des relativen Energieumsatzes lassen sich bei der Sauerstoffaufnahme Differenzen bzgl. der Zuordnung zu den Leistungen in Watt erkennen. Bei ähnlich hoher Sauerstoffaufnahme kann es zu unterschiedlichen Leistungen zwischen den Forstwirten kommen. Jedoch lässt sich auch feststellen, dass in allen drei Messzeitpunkten die Zuordnung bei jedem einzelnen Forstwirt die Leistungen in Watt in Relation zur Sauerstoffaufnahme stehen (eine höhere Sauerstoffaufnahme bedingt eine höhere Leistung in Watt). 


\subsection{Energiebilanz}

Mittels der erhobenen Werte aus den Ernährungs- und Aktivitätsprotokollen kann aus der Differenz von Energieaufnahme und Energieumsatz die Energiebilanz ermittelt werden. In Tabelle 36 werden die Bilanzen für die jeweils gesamte Woche, für die Werktage von Montag bis Donnerstag, für die Wochenendtage Samstag und Sonntag sowie für die gemittelten Werte für einen einzelnen Tag einer gesamten Woche in $\mathrm{t} 1$ - $\mathrm{t} 3$ abgebildet.

Tabelle 36: Energiebilanz in $\mathbf{t} 1, \mathrm{t} 2$ und $\mathrm{t} 3$.

\begin{tabular}{|lr|r|r|r|r|}
\hline \hline & \multicolumn{1}{|c|}{ N } & \multicolumn{1}{c|}{ Minimum } & Maximum & \multicolumn{1}{c|}{ Mittelwert } & Standardabweichung \\
\hline $\begin{array}{l}\text { Mittelwert der Energiebilanz in einer Woche } \\
\text { aller Probanden aller Messzeitpunkte }\end{array}$ & 14 & $-10558,00$ & $-1804,00$ & $-6957,5714$ & 3059,83908 \\
\hline Energiebilanz aller Probanden in t1 & 14 & $-13073,00$ & 3625,00 & $-5264,4286$ & 6148,87802 \\
\hline Energiebilanz aller Probanden in t2 & 14 & $-12670,00$ & $-2678,00$ & $-8026,7143$ & 2713,84800 \\
\hline Energiebilanz aller Probanden in t3 & 14 & $-10213,00$ & $-1156,00$ & $-7581,9286$ & 2244,89655 \\
\hline $\begin{array}{l}\text { Mittelwert der Energiebilanzen an Werktagen } \\
\text { (Tag 1 bis Tag 4) in t1 }\end{array}$ & 14 & $-502,00$ & 202,00 & $-110,7143$ & 214,27137 \\
\hline $\begin{array}{l}\text { Mittelwert der Energiebilanzen an Werktagen } \\
\text { (Tag 1 bis Tag 4) in t2 }\end{array}$ & 14 & $-712,00$ & $-122,00$ & $-425,5000$ & 150,86150 \\
\hline $\begin{array}{l}\text { Mittelwert der Energiebilanzen an Werktagen } \\
\text { (Tag 1 bis Tag 4) in t3 }\end{array}$ & 14 & $-789,00$ & $-45,00$ & $-390,5714$ & 188,48208 \\
\hline $\begin{array}{l}\text { Mittelwert der Energiebilanzen am Wochenende } \\
\text { (Tag 6 und Tag 7) in t1 }\end{array}$ & 14 & $-348,00$ & 1190,00 & 356,0714 & 593,41404 \\
\hline $\begin{array}{l}\text { Mittelwert der Energiebilanzen am Wochenende } \\
\text { (Tag 6 und Tag 7) in t2 }\end{array}$ & 14 & $-632,00$ & 778,00 & $-2,3571$ & 468,92949 \\
\hline $\begin{array}{l}\text { Mittelwert der Energiebilanzen am Wochenende } \\
\text { (Tag 6 und Tag 7) in t3 }\end{array}$ & 14 & $-859,00$ & 1111,00 & $-19,5000$ & 613,82066 \\
\hline Mittelwert der Energiebilanz pro Tag in t1 & 14 & $-1868,00$ & 518,00 & $-752,0714$ & 878,41916 \\
\hline Mittelwert der Energiebilanz pro Tag in t2 & 14 & $-1810,00$ & $-383,00$ & $-1146,6429$ & 387,56788 \\
\hline Mittelwert der Energiebilanz pro Tag in t3 & 14 & $-1459,00$ & $-165,00$ & $-1083,1429$ & 320,72127 \\
\hline Gültige Werte (Listenweise) & 14 & 14 & 14 & 14 & 14 \\
\hline \hline
\end{tabular}

Auffällig ist, dass sich alle Werte der Gesamtbilanz im negativen Bereich befinden und somit eine negative Energiebilanz in allen drei Messzeitpunkten vorliegt. Die negativste Energiebilanz liegt in $\mathrm{t} 2$ mit einem Wert von $-8026 \mathrm{kcal}$ vor. Der durchschnittliche Energiebilanzwert in $\mathrm{t} 3$ von $-7581 \mathrm{kcal}$ beschreibt eine ähnlich negative Energiebilanz. In t1 ist ebenfalls ein negativer Wert mit -5264 kcal zu verzeichnen. Somit liegt die durchschnittliche Energiebilanz aller drei Messzeitpunkte bei $-6957 \mathrm{kcal}$.

Die durchschnittliche Energiebilanz pro Tag ist im Winter am Höchsten (-752 kcal). Eine niedrigere Bilanz wird im Sommer erreicht (-1083 kcal). Im Frühjahr ist sie mit $-1146 \mathrm{kcal}$ am Niedrigsten. Es zeigt sich also, dass sich die Energiebilanzen unterscheiden. Bei der Energiebilanz pro Woche bestätigen sich aus rechnerischen Gründen die Zahlen.

Tatsächlich zeigen sich auch signifikante Unterschiede, jedoch nur zwischen dem Winter (t1) und dem Frühjahr (t2) $(\mathrm{p}<=0,05)$. Zwischen Winter und Sommer bzw. Frühling und Sommer gibt es keine signifikanten Differenzen. Aufgrund der hohen Standardabweichung und der 
geringen Fallzahl können jedoch keine aussagekräftigen Ergebnisse bzgl. der Signifikanzen getätigt werden.

Bei der Betrachtung der Energiebilanz einzelner Tage ist auffällig, dass lediglich die Energiebilanz am Wochenende in $\mathrm{t} 1 \mathrm{mit} 356 \mathrm{kcal}$ einen positiven Wert erreicht. Die Werte am Wochenende in $\mathrm{t} 2$ und $\mathrm{t} 3$ spiegeln eine nahezu ausgeglichene Energiebilanz mit $-2 \mathrm{kcal}$ und -19 kcal wider. An den Werktagen Montag bis Donnerstag konnte in allen drei Messzeitpunkten eine negative Energiebilanz mit Werten von $-110 \mathrm{kcal}$ in $\mathrm{t} 1,-425 \mathrm{kcal}$ in $\mathrm{t} 2$ und $-390 \mathrm{kcal}$ in $\mathrm{t} 3$ errechnet werden.

In Abbildung 29 wird mittels linearer Regression die Energiebilanz der einzelnen Forstwirte in allen drei Messzeitpunkten dargestellt. Die konstante Steigung beschreibt die Linie, auf der eine ausgewogene Energiebilanz abgebildet wird.

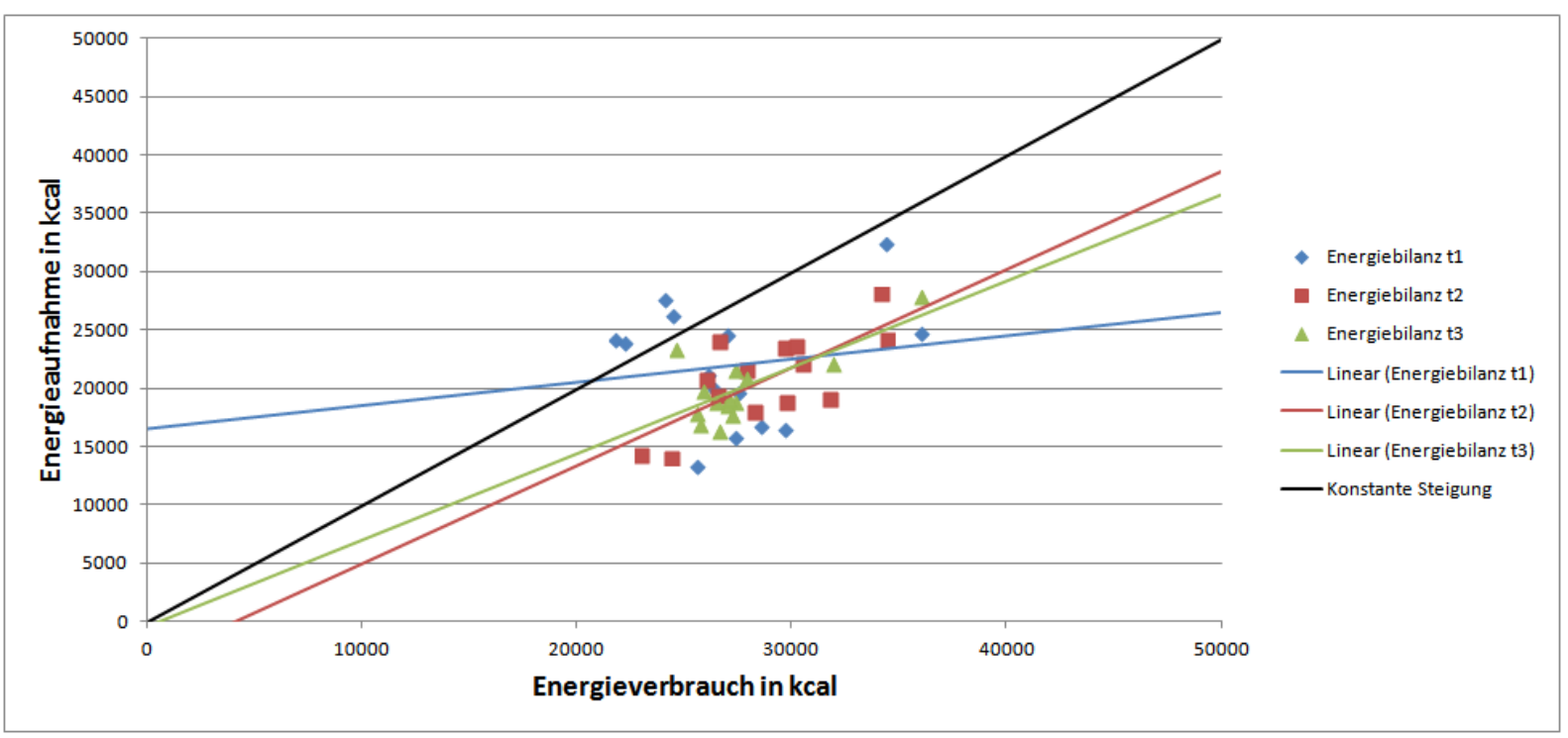

Abbildung 29: Energiebilanz in kcal.

Es lässt sich ablesen, dass lediglich bei vier Forstwirten positive Energiebilanzen vorliegen, die alle in $\mathrm{t} 1$ fallen. Der Verlauf der Bilanzen in $\mathrm{t} 2$ und $\mathrm{t} 3$ ist ähnlich und verläuft fast parallel zur konstanten Steigung $\mathrm{m}=1$, die eine ausgewogene Energiebilanz abbildet. Im Kern (Energieverbrauch zwischen $27000 \mathrm{kcal}$ und $33000 \mathrm{kcal}$ ) verlaufen die Energiebilanzen von t2 und t3 sehr ähnlich. In t1 ist eine starke Streuung zu verzeichnen.

Beim T-Test zwischen den Energiebilanzen (vgl. Tabelle 37) zeigen sich lediglich zwischen $\mathrm{t} 1$ und $\mathrm{t} 2$ signifikante Unterschiede $(\mathrm{p}<=0,05)$. Zwischen $\mathrm{t} 1$ und $\mathrm{t} 3$ und zwischen $\mathrm{t} 2$ und $\mathrm{t} 3$ gibt es keine signifikanten Differenzen. 
Tabelle 37: T-Test zwischen den Mittelwerten der Energiebilanzen in t1, t2 und t3.

\begin{tabular}{|c|c|c|c|c|c|c|c|c|c|}
\hline \multicolumn{10}{|c|}{ Test bei gepaarten Stichproben } \\
\hline & & \multicolumn{5}{|c|}{ Gepaarte Differenzen } & \multirow[t]{3}{*}{$\mathrm{T}$} & \multirow[t]{3}{*}{$\mathrm{df}$} & \multirow{3}{*}{$\begin{array}{l}\text { Sig. (2- } \\
\text { seitig) }\end{array}$} \\
\hline & & \multirow[t]{2}{*}{ Mittelwert } & \multirow{2}{*}{$\begin{array}{l}\text { Stan- } \\
\text { dardabwei- } \\
\text { chung }\end{array}$} & \multirow{2}{*}{$\begin{array}{l}\text { Standardfehler } \\
\text { des Mittelwer- } \\
\text { tes }\end{array}$} & \multicolumn{2}{|c|}{$\begin{array}{l}\text { 95\% Konfidenzintervall der } \\
\text { Differenz }\end{array}$} & & & \\
\hline & & & & & Untere & Obere & & & \\
\hline $\begin{array}{l}\text { Paaren } \\
1\end{array}$ & $\begin{array}{l}\text { MW der Energie- } \\
\text { bilanz pro Tag in } \mathrm{t} 1- \\
\text { MW der Energie- } \\
\text { bilanz pro Tag in } \mathrm{t} 2\end{array}$ & 394,57143 & 652,51145 & 174,39102 & 17,82254 & 771,32032 & 2,263 & 13 &, 041 \\
\hline $\begin{array}{l}\text { Paaren } \\
2\end{array}$ & $\begin{array}{l}\text { MW der Energie- } \\
\text { bilanz pro Tag in } \mathrm{t} 1- \\
\text { MW der Energie- } \\
\text { bilanz pro Tag in } \mathrm{t} 3\end{array}$ & 331,07143 & 855,10237 & 228,53572 & $-162,64998$ & 824,79284 & 1,449 & 13 & ,171 \\
\hline $\begin{array}{l}\text { Paaren } \\
3\end{array}$ & $\begin{array}{l}\text { MW der Energie- } \\
\text { bilanz pro Tag in } \mathrm{t} 2- \\
\text { MW der Energie- } \\
\text { bilanz pro Tag in } \mathrm{t} 3\end{array}$ & $-63,50000$ & 444,47112 & 118,78990 & $-320,12998$ & 193,12998 &,- 535 & 13 & ,602 \\
\hline
\end{tabular}

Ein Zusammenhang zwischen Energiebilanz und Energieumsatz ist zu erwarten, da der Energieumsatz eine Variable für die Berechnung der Energiebilanz darstellt. Nichtsdestotrotz werden in Tabelle 38 die signifikanten Unterschiede bei den Korrelationen zwischen Energiebilanz und Energieumsatz dargestellt, um feststellen zu können, ob die Energieaufnahme an den Energieumsatz angepasst wird und somit eine ausgeglichene Energiebilanz verzeichnet werden kann. 
Tabelle 38: Korrelationen zwischen der Energiebilanz und dem Energieumsatz.

\begin{tabular}{|c|c|c|c|c|c|c|c|c|c|}
\hline \multicolumn{10}{|c|}{ Korrelationen an Werktagen } \\
\hline & & $\begin{array}{l}\text { Energie- } \\
\text { bilanz } \\
\text { aller } \\
\text { Proban- } \\
\text { den in t1 }\end{array}$ & $\begin{array}{l}\text { Mittelwert } \\
\text { der Ener- } \\
\text { giebilanzen } \\
\text { an Werkta- } \\
\text { gen in t1 }\end{array}$ & $\begin{array}{l}\text { Mittelwert } \\
\text { der Ener- } \\
\text { giebilanzen } \\
\text { an Werkta- } \\
\text { gen in } \mathrm{t} 2\end{array}$ & $\begin{array}{l}\text { Mittelwert } \\
\text { der Ener- } \\
\text { giebilanzen } \\
\text { an Werkta- } \\
\text { gen in t3 }\end{array}$ & $\begin{array}{l}\text { Mittelwert } \\
\text { der Ener- } \\
\text { giebilanzen } \\
\text { am Wo- } \\
\text { chenende in } \\
\text { t2 }\end{array}$ & $\begin{array}{c}\text { Ener- } \\
\text { gieum- } \\
\text { satz an } \\
\text { Werk- } \\
\text { tagen in } \\
\text { t1 }\end{array}$ & $\begin{array}{c}\text { Ener- } \\
\text { gieum- } \\
\text { satz am } \\
\text { Wo- } \\
\text { chen- } \\
\text { ende in } \\
\text { t1 }\end{array}$ & $\begin{array}{l}\text { Energieum- } \\
\text { satz am } \\
\text { Wochenen- } \\
\text { de in } 22\end{array}$ \\
\hline $\begin{array}{l}\text { Energiebi- } \\
\text { lanz aller } \\
\text { Probanden } \\
\text { in } \mathrm{t} 2\end{array}$ & $\begin{array}{l}\text { Korrelation nach Pearson } \\
\text { Signifikanz (2-seitig) } \\
\mathrm{N}\end{array}$ & $\begin{array}{r}, 729 \\
, 003 \\
14\end{array}$ & & & & & & & \\
\hline $\begin{array}{l}\text { Mittelwert } \\
\text { der Ener- } \\
\text { giebilanzen } \\
\text { an Werkta- } \\
\text { gen in } \mathrm{t} 2 \\
\end{array}$ & $\begin{array}{l}\text { Korrelation nach Pearson } \\
\text { Signifikanz (2-seitig) } \\
\mathrm{N}\end{array}$ & & $\begin{array}{r}708 \\
, 005 \\
14\end{array}$ & & & & & & \\
\hline $\begin{array}{l}\text { Mittelwert } \\
\text { der Ener- } \\
\text { giebilanzen } \\
\text { an Werkta- } \\
\text { gen in } \mathrm{t} 3\end{array}$ & $\begin{array}{l}\text { Korrelation nach Pearson } \\
\text { Signifikanz (2-seitig) } \\
\mathrm{N}\end{array}$ & & $\begin{array}{r}, 575 \\
, 032 \\
14\end{array}$ & & & & & & \\
\hline $\begin{array}{l}\text { Mittelwert } \\
\text { der Ener- } \\
\text { giebilanzen } \\
\text { am Wo- } \\
\text { chenende in } \\
\text { t3 }\end{array}$ & $\begin{array}{l}\text { Korrelation nach Pearson } \\
\text { Signifikanz (2-seitig) } \\
\text { N }\end{array}$ & & & & & $\begin{array}{r}, 641 \\
, 013 \\
14\end{array}$ & & & \\
\hline $\begin{array}{l}\text { Energieum- } \\
\text { satz an } \\
\text { Werktagen } \\
\text { in } \mathrm{t} 1\end{array}$ & $\begin{array}{l}\text { Korrelation nach Pearson } \\
\text { Signifikanz (2-seitig) } \\
\text { N }\end{array}$ & & $\begin{array}{r}-, 640 \\
, 014 \\
14\end{array}$ & $\begin{array}{r}-, 585 \\
, 028 \\
14\end{array}$ & $\begin{array}{r}-, 642 \\
, 013 \\
14\end{array}$ & & & & \\
\hline $\begin{array}{l}\text { Energieum- } \\
\text { satz an } \\
\text { Werktagen } \\
\text { in } \mathrm{t} 2\end{array}$ & $\begin{array}{l}\text { Korrelation nach Pearson } \\
\text { Signifikanz (2-seitig) } \\
\text { N }\end{array}$ & & & $\begin{array}{r}-, 696 \\
, 006 \\
14\end{array}$ & & $\begin{array}{r}569 \\
, 034 \\
14\end{array}$ & $\begin{array}{r}, 540 \\
, 046 \\
14\end{array}$ & & \\
\hline $\begin{array}{l}\text { Energieum- } \\
\text { satz an } \\
\text { Werktagen } \\
\text { in } \mathrm{t} 3\end{array}$ & $\begin{array}{l}\text { Korrelation nach Pearson } \\
\text { Signifikanz (2-seitig) } \\
\text { N }\end{array}$ & & $\begin{array}{r}-, 568 \\
, 034 \\
14\end{array}$ & & $\begin{array}{r}-, 796 \\
, 001 \\
14\end{array}$ & & $\begin{array}{r}779 \\
, 001 \\
14\end{array}$ & & \\
\hline $\begin{array}{l}\text { Energieum- } \\
\text { satz am } \\
\text { Wochenen- } \\
\text { de in } \mathrm{t} 2\end{array}$ & $\begin{array}{l}\text { Korrelation nach Pearson } \\
\text { Signifikanz (2-seitig) } \\
\text { N }\end{array}$ & & & & & & & $\begin{array}{r}, 670 \\
, 009 \\
14\end{array}$ & \\
\hline $\begin{array}{l}\text { Energieum- } \\
\text { satz am } \\
\text { Wochenen- } \\
\text { de in } \mathrm{t} 3\end{array}$ & $\begin{array}{l}\text { Korrelation nach Pearson } \\
\text { Signifikanz (2-seitig) } \\
\mathrm{N}\end{array}$ & & & & & & & $\begin{array}{r}, 606 \\
, 022 \\
14\end{array}$ & $\begin{array}{r}734 \\
, 003 \\
14\end{array}$ \\
\hline
\end{tabular}

Der Tabelle 38 ist zu entnehmen, dass in allen drei Messzeitpunkten die Energiebilanz mit dem Energieverbrauch korreliert. Dies bedeutet, dass bei einer positiveren Energiebilanz ein entsprechend geringerer Wert vorliegt. Somit kann Hypothese 4 verifiziert werden, die besagt, dass die Energiebilanz negativ mit dem Energieumsatz bei der beruflichen Aktivität korreliert. Die Bilanzierungen der Werktage korrelieren jeweils hoch untereinander. Die Energiebilanz der Werktage in t1 korrelieren negativ hoch mit der Aktivität der Werktage. Das bedeutet, dass vermehrte Aktivität im Winter sich „,negativ“ auf die Energiebilanz auswirkt. Auch für die Werktage in $\mathrm{t} 2$ kann eine negative Korrelation zwischen Bilanz und Aktivität verzeichnet werden, die jedoch etwas geringer ausfällt. Und auch in t3 zeigt sich, dass Bilanz und Aktivi- 
tät korrelieren. An den Wochenenden zeigen sich jedoch durchweg positive, starke signifikante Zusammenhänge, d. h. mit steigender Aktivität und einem höheren Energieumsatz erhöht sich auch die Energiebilanz. 


\section{DISKUSSION}

Zielsetzung der vorliegenden Studie war die Erfassung der Energiebilanz bei Forstwirten zu unterschiedlichen Jahreszeiten bzw. in beruflichen Anforderungssituationen. Mittels Ernährungs- und Aktivitätsprotokollen und indirekter Kalorimetrie wurden Werte zur Bestimmung der Energiebilanz erhoben. Um weitere Einflussfaktoren auf die Energiebilanz berücksichtigen zu können, werden soziodemografische und anthropometrische Daten, äußere Bedingungen und die Körperkomposition mittels Kalipermetrie ermittelt. Es wird das Zusammenwirken der Energiebilanz, der Ernährungssituation und der individuellen Belastungssituation der Forstwirte analysiert.

\section{Diskussion der Ergebnisse}

In den folgenden Kapiteln werden die gewonnenen Ergebnisse der Studie interpretiert und unter Berücksichtigung des aktuellen Forschungsstandes diskutiert. Eine Erörterung der Untersuchungsmethodik wird vorgenommen und knüpft an die Diskussion der Ergebnisse an.

\subsection{Anthropometrische Daten}

Anthropometrische Daten werden zum einen mittels der Körpermaße (BMI) und zum anderen mit Hilfe der Kalipermetrie, die den Körperfettanteil bestimmt, ermittelt. Die BMI-Werte der Probanden liegen mit 26,9 in $\mathrm{t} 1$ und $\mathrm{t} 2$ und 27,3 in $\mathrm{t} 3$ laut WHO-Definition im übergewichtigen Bereich, der die Werte zwischen 25 und 30 umfasst. Diese Werte liegen im durchschnittlichen Bereich der männlichen deutschen Bevölkerung, der in der Literatur zwischen 26,3 und 26,9 angegeben wird (vgl. Kapitel 4.2). Die BMI-Werte entsprechen somit dem Durchschnitt der deutschen männlichen Bevölkerung. Aufgrund des Anforderungsprofils der Forstwirte lässt sich vermuten, dass die erhöhten BMI-Werte in einer größeren Muskelmasse begründet liegen. Durch die Kalipermetrie wurde von der subkutanen Fettschichtdicke auf den Körperfettanteil geschlossen. Der gemessene Körperfettanteil lässt sich mit ca. $20 \%$ im Übergang von Normalgewicht zu Adipositas einordnen, da zwischen 20 und $25 \%$ der adipöse Bereich definiert ist (de Marées 2003).

Bzgl. der Entwicklung des Körpergewichts (und somit des BMI) und des Körperfettanteils lässt sich konstatieren, dass eine Zunahme von $\mathrm{t} 1 \mathrm{zu} \mathrm{t} 3$ stattfindet. In $\mathrm{t} 1$ beträgt das durchschnittliche Körpergewicht $87 \mathrm{~kg}$. Aus organisatorischen Gründen konnte die Kalipermetrie in $\mathrm{t} 1$ nicht durchgeführt werden. In $\mathrm{t} 2$ betragen das durchschnittliche Körpergewicht $88 \mathrm{~kg}$ und der durchschnittliche Körperfettanteil 19,36 \%. Diese Werte steigen in t3 auf ein durch- 
schnittliches Körpergewicht von $89 \mathrm{~kg}$ und einen durchschnittlichen Körperfettanteil von 20,81\%. Dieses Ergebnis steht im Gegensatz zu den von Ma (2006) erhobenen Werten, die bei knapp 600 Probanden in einem Zeitraum von einem Jahr feststellen konnten, dass die Probanden im Winter ein um 0,5 kg höheres Körpergewicht haben.

Diese Gewichtszunahme lässt sich nicht auf die erhobene (negative) Energiebilanz der Probanden zurückführen, so dass in Kapitel 8.6 ausführlicher auf mögliche Ursachen dieser Diskrepanz eingegangen wird. Allgemein lässt sich festhalten, dass sich der erhöhte BMI der Probanden auf die anstrengenden körperlichen Tätigkeiten zurückführen lässt und auf die daraus resultierende größere Muskelmasse. Diese Aussage wird jedoch nicht durch die erhobenen Körperfettanteile bestätigt.

\subsection{Energieaufnahme}

Die Energieaufnahme wurde in allen drei Messzeitpunkten mittels eines einwöchigen Ernährungsprotokolls geführt. Die Auswertung mit der Software DGE-PC liefert Daten zur Zusammensetzung der aufgenommenen Nahrung und ermöglicht einen Vergleich zwischen der Nahrungsaufnahme an Wochentagen und am Wochenende, wobei der Freitag aufgrund seiner gesonderten Struktur nicht in diesen Vergleich einfließt.

Die Energieaufnahme in der Woche ist im Winter in t1 mit 21948 kcal/ Woche am Höchsten. Im Frühjahr in $\mathrm{t} 2$ beträgt die Energieaufnahme $20801 \mathrm{kcal} /$ Woche und im Sommer in $\mathrm{t} 3$ wurde ein Wert von durchschnittlich $20017 \mathrm{kcal} /$ Woche erhoben. Diese Werte ergeben, dass im Winter im Vergleich zum Frühjahr eine Mehraufnahme von ca. 164 kcal/ d und im Vergleich zum Sommer eine Mehraufnahme von ca. $275 \mathrm{kcal} / \mathrm{d}$ vorliegen. Diese saisonalen Differenzen übersteigen bereits vorhandene Ergebnisse. So erhob Ma (2006) eine Mehraufnahme im Herbst im Vergleich zum Frühjahr von 86 kcal/ d. Bei dieser Stichprobe handelt es sich jedoch nicht um Personen, die schwere körperliche Arbeit verrichten. Weitere Untersuchungen zeigen saisonale Unterschiede in der Energieaufnahme auf (Schneider \& Heseker 2006; Westerterp, Plasqui \& Goris 2005). Allerdings liegt hier eine niedrigere Energieaufnahme im Winter vor. Die Differenzen in der Berufsgruppe der Forstwirte lassen vermuten, dass die Energieaufnahme an die unterschiedlichen jahreszeitlichen Tätigkeiten angepasst ist. Dieser Aspekt wird in Kapitel 8.6 ausführlicher diskutiert. Darüber hinaus kann ein Effekt hinsichtlich der Compliance der Probanden angedacht werden. Bei einem längeren Erhebungszeitraum als einer Woche mit einem Ernährungsprotokoll konnte festgestellt werden, dass Probanden zu unvollständigeren Angaben und zu einfachen Gerichten neigen (Schneider \& Heseker 2006). Dieser Aspekt kann auf das wiederholte Führen eines Protokolls übertragen wer- 
den, so dass die stark differierenden Werte relativiert werden. Aufgrund der zwar dreigeteilten aber dennoch dreiwöchigen Protokollierung ist anzunehmen, dass die Probanden von t1 zu t3 die Protokollierung z. T. nachlässiger durchgeführt haben. Darüber hinaus ist die personale Variation bei der Energieaufnahme recht hoch (Cai et al. 2004), so dass bei der Betrachtung der erhobenen Werte immer berücksichtigt werden muss, dass die Energieaufnahme in der gemessenen Woche durchaus von der Energieaufnahme der angrenzenden Wochen abweichen kann. Nichts desto trotz bleibt eine Differenz zwischen der Energieaufnahme zu den unterschiedlichen Jahreszeiten bestehen.

Ordnet man die erhobenen Daten der Forstwirte den Richtwerten für die Energiezufuhr (Hollmann \& Hettinger 2000; Kasper 2000) zu, so ergibt sich, dass die durchschnittliche tägliche Energieaufnahme der Forstwirte von $2988 \mathrm{kcal} / \mathrm{d}$ dem Richtwert für Mittelschwerarbeiter entspricht. Dies lässt sich gut mit dem Anforderungsprofil des Berufes verknüpfen, das eine hohe körperliche Beanspruchung der Forstwirte impliziert. Eine genauere Betrachtung des Energieumsatzes bei den gemessenen beruflichen Tätigkeiten findet in Kapitel 8.3 statt.

Stellt man die tägliche Energieaufnahme an Werktagen der täglichen Energieaufnahme am Wochenende gegenüber, so lässt sich erkennen, dass in allen drei Messzeitpunkten die Energieaufnahme am Wochenende über der Energieaufnahme an Werktagen liegt. Diese Differenz ist in $\mathrm{t} 1 \mathrm{mit}$ einem Wert von $518 \mathrm{kcal} / \mathrm{d}$ am größten und in $\mathrm{t} 3$ mit einer Differenz von $88 \mathrm{kcal} /$ $\mathrm{d}$ am geringsten. In $\mathrm{t} 2$ liegt eine Differenz von $153 \mathrm{kcal} / \mathrm{d}$ vor. Der in Studien gemessene geringe Unterschied zwischen der Energieaufnahme an Wochentagen und am Wochenende (Hartman et al. 1990; Cai et al. 2004; Schneider \& Heseker 2006) zeichnet sich in den Daten der vorliegenden Studie mit einem deutlicheren Unterschied ab. Ein Erklärungsansatz wäre, dass die Energieaufnahme an Werktagen für die starke körperliche Arbeit nicht ausreicht und deshalb am Wochenende eine vermehrte Nahrungsaufnahme stattfindet, um die negative Energiebilanz der Arbeitstage auszugleichen. Eine weitere Erklärung bietet eine Betrachtung aus der sozialen und psychologischen Perspektive, die die vermehrte Energiezufuhr am Wochenende durch Essen im sozialen Gefüge der Familie oder Freunde ohne zeitliche und emotionale Belastung durch den Beruf begründet.

Bei der Zusammensetzung der Nahrung liegen Mittelwerte von allen drei Messzeitpunkten vor, die einen Eiweißanteil von $16 \%$, einen Kohlenhydratanteil von $43 \%$ und einen Fettanteil von $37 \%$ belegen. Bei der Evaluierung saisonaler Tendenzen werden ein leicht erhöhter Kohlenhydratanteil von $45 \%$ im Winter in t1 und ein leicht erhöhter Fettanteil von 39 \% im Frühjahr in $\mathrm{t} 2$ deutlich. Diese Ergebnisse stehen im Kontrast zu den Ergebnissen der Studie von Ma (2006), in der eine erhöhte Kohlenhydratzufuhr im Frühjahr verzeichnet werden konnte. 
Geht man von einer bedarfsangepassten Ernährung aus, so könnte man darauf schließen, dass der erhöhte Kohlenhydratanteil im Winter in $\mathrm{t} 1$ auf eine vermehrte körperliche Aktivität der Probanden zurückzuführen ist. Um den daraus resultierenden erhöhten Energiebedarf zu decken, findet eine unbewusste vermehrte Kohlenhydratzufuhr statt. Jedoch lässt sich bei der Betrachtung der Energieumsätze feststellen, dass in t1 der niedrigste Energieumsatz vorliegt (vgl. Kapitel 8.4), so dass diese Schlussfolgerung nicht bestätigt werden kann.

Zielführender ist hier ein Vergleich zwischen der empfohlenen und der tatsächlichen Zusammensetzung der Nahrung. Der Eiweißanteil liegt mit $16 \%$ über dem empfohlenen Anteil von $10 \%$. Dieser erhöhte Anteil geht vermutlich mit der erhöhten körperlichen Aktivität der Forstwirte einher, so dass der durchschnittliche Eiweißanteil von $16 \%$ dadurch legitimiert wird. Durch diesen Mehranteil an Proteinen wird gewährleistet, dass trotz der hohen körperlichen Aktivität der Ersatz und Neuaufbau eiweißhaltiger Substanzen gewährleistet wird. Der Eiweißanteil liegt außerdem unter dem Eiweißanteil der Zivilisationskost, der $20 \%$ beträgt. Der erhöhte Eiweißanteil stimmt mit dem erhobenen erhöhten Eiweißanteil bei griechischen Forstwirten überein (Gallis \& Panagopoulou 2007). Somit lässt sich festhalten, dass der Eiweißanteil der Probanden mit hoher körperlicher Beanspruchung im Berufsalltag dem Bedarf angepasst zu sein scheint. Eine Zufuhr proteinhaltiger Nahrung findet optimal direkt nach der Belastung statt, um den Erhalt der Muskelkraft und die muskuläre Erholung zu unterstützen.

Der Kohlenhydratanteil liegt mit durchschnittlich $43 \%$ deutlich unter dem empfohlenen Anteil von $60 \%$, aber dennoch über dem Anteil der Zivilisationskost von $35 \%$. Aufgrund der hohen körperlichen Aktivität ist ein größerer Anteil an Kohlenhydraten notwendig, um den Energiebedarf zu decken, der bei steigender körperlicher Aktivität vermehrt aus Kohlenhydraten gewonnen wird. Eine Tendenz im Vergleich zur Zivilisationskost ist hier bereits zu erkennen, sollte aber durch eine Erhöhung des Kohlenhydratanteils und eine Anpassung an die körperliche Beanspruchung optimiert werden.

Der Fettanteil der aufgenommenen Nahrung der Probanden liegt mit $37 \%$ zwischen dem empfohlenen Anteil von $30 \%$ und dem Anteil der Zivilisationskost von $45 \%$. Bei erhöhter körperlicher Aktivität wird eine Reduktion des Fettanteils empfohlen (de Marées 2003; Konopka 2009), um einen größeren Anteil an Kohlenhydraten zu ermöglichen. Lediglich bei Schwerstarbeit sei eine fettreiche Nahrungszufuhr notwendig, um den sehr hohen Energieverbrauch decken zu können (Golenhofen 2006). Da es sich bei der Tätigkeit der Forstwirte im Durchschnitt nicht um Schwerstarbeit handelt, sondern vielmehr um eine mittelschwere bis schwere Arbeit, sollte der Kohlenhydratanteil der Nahrungsaufnahme an die körperliche Be- 
anspruchung angepasst und auf Kosten des Fettanteils erhöht werden. Auf diese Weise wird der für den Energiegewinn notwendige Anteil an Kohlenhydraten zur Verfügung gestellt.

Durch eine an die Bedürfnisse der beruflichen Belastung der Forstwirte angepasste Ernährung in Form einer fettreduzierten und kohlenhydratangereicherten Ernährung werden aus ökotrophologischer Sicht optimale Bedingungen für die Leistungsfähigkeit geschaffen.

Abschließend sei zu erwähnen, dass die Zusammensetzung der Nahrungszufuhr großen interpersonalen Schwankungen unterliegt. So kann z. B. für FW08 festgehalten werden, dass er in allen drei Messzeitpunkten einen verhältnismäßig geringen Anteil an Fett zu sich nimmt. Die erhobenen Daten verdeutlichen die Relevanz einer individuellen Ernährungsberatung, die auf den Grundlagen einer forstwirtgerechten Ernährung basiert, jedoch den Bedürfnissen jedes einzelnen Probanden angepasst ist.

\subsection{Flüssigkeitszufuhr}

Ein ausgewogener Wasserhaushalt ist ein weiterer Faktor für die Leistungsfähigkeit des Menschen (Manore 2004; Konopka 2009), weshalb mittels der Ernährungsprotokolle ebenfalls die Flüssigkeitszufuhr erhoben wurde. Mit einem durchschnittlichen Wasserkonsum von 3,5 1/ d liegen die Forstwirte mit ihrer Flüssigkeitszufuhr über der empfohlenen Menge von $1 \mathrm{ml} / \mathrm{Ka}-$ lorie Energieaufnahme bei mittlerer Temperatur und normaler körperlicher Arbeit. Dieser Empfehlung zufolge beträgt die optimale Flüssigkeitsaufnahme ca. 3 1/ d bei einer Energieaufnahme von durchschnittlich $2988 \mathrm{kcal} / \mathrm{d}$, wobei 11 Flüssigkeit über Lebensmittel und Stoffwechselwasser aufgenommen wird, so dass sich eine optimale Flüssigkeitszufuhr von 2 1/ d ergibt. Die um 0,5 1 höhere Flüssigkeitszufuhr der Forstwirte lässt sich durch die hohe körperliche Aktivität im Berufsalltag begründen, an die die eingenommene Flüssigkeitsmenge angepasst zu sein scheint. Mit diesem Argument lässt sich auch die etwas geringere durchschnittliche Flüssigkeitszufuhr am Wochenende von 3,2 1/ d erläutern. Da die körperliche Aktivität der Forstwirte aufgrund der wegfallenden beruflichen Tätigkeit am Wochenende geringer ist als an Werktagen, sinkt der Flüssigkeitsbedarf. Eine Erhöhung der Flüssigkeitszufuhr im Sommer in $\mathrm{t} 3$ kann nicht festgestellt werden, so dass eine Anpassung an die Umgebungstemperatur nicht beobachtet werden kann. Qualitativ ist festzuhalten, dass die Forstwirte größtenteils Wasser und Früchte-/ Kräutertee konsumieren und somit der allgemeinen Empfehlung nachkommen, bei körperlicher Belastung die Flüssigkeit überwiegend über mineralstoffreiche Getränke zu sich zu nehmen. Ein verbesserungswürdiger Punkt ist sicherlich die Zufuhr über flüssigkeitsreiches Obst, da Obst nur von wenigen Forstwirten regelmäßig verzehrt wird. 
Die Betrachtung des Alkoholkonsums ergibt, dass am Wochenende im Vergleich zu den Werktagen das bis zu Zehnfache an Alkohol konsumiert wird. In t1 beträgt die durchschnittliche Alkoholmenge an Werktagen $3 \mathrm{~g} / \mathrm{d}$ und am Wochenende 33,4 g/ d. In t2 liegt eine Alkoholmenge von 4,7 g/ d an Werktagen und 18,3 g/ d am Wochenende vor. In $\mathrm{t} 3$ wird an Werktagen mit 7,7 g/ d der meiste Alkohol und am Wochenende mit 14,7 g/ d die geringste Alkoholmenge konsumiert. Der Mittelwert der ganzen Woche ergibt mit 15,2 g/ d in t3 den höchsten Wert und mit 10,1 g/ d in t2 den niedrigsten Wert. Die erhöhte Alkoholmenge am Wochenende ist selbsterklärend mit einhergehenden Feierlichkeiten und Anlässen. Der höchste Alkoholkonsum bzgl. der Werktage in $\mathrm{t} 3$ im Sommer lässt sich dadurch erklären, dass Bier den Hauptkonsum ausmacht, welches bei warmen Temperaturen als Getränk am Abend konsumiert wurde. Dies spiegelt sich auch darin wider, dass der durchschnittliche Wert der gesamten Woche in $\mathrm{t} 3$ am höchsten ist und dass $\mathrm{t} 3$ der einzige Messzeitpunkt ist, in dem alle Probanden Alkohol zu sich genommen haben.

Im Vergleich zu den empfohlenen Grenzwerten des Alkoholkonsums, der für Männer bei 24 g/ d liegt, liegen die Mittelwerte der Probanden unter diesem Grenzwert. Zu berücksichtigen ist jedoch, dass bei einer individuellen Betrachtung der Ergebnisse bzgl. des Konsums alkoholischer Getränke bei Probanden an einzelnen Tagen Werte erfasst wurden, die diesen Grenzwert z. T. deutlich übersteigen. Um jedoch eine fundierte Aussage über den Konsum alkoholischer Getränke machen zu können, wäre eine Protokollierung über einen längeren Zeitraum notwendig, der eine individuelle Analyse des Alkoholkonsums der einzelnen Probanden zuließe.

\subsection{Energieumsatz}

Der Energieumsatz wurde mittels eines Aktivitätsprotokolls geschätzt, anhand dessen die Probanden zu jedem Messzeitpunkt über einen Erhebungszeitraum einer Woche in 15minütigen Intervallen ihre verrichtete Tätigkeit angekreuzt haben. Diese ausführliche Protokollierung ermöglichte bei der Auswertung eine Erhebung des Energieumsatzes während beruflicher Tätigkeit, während der Freizeit und einen Vergleich des Energieumsatzes an Werktagen und am Wochenende. Für jeden Tätigkeitsbereich wurde auf der Basis bereits erhobener Daten ein Multiplikationsfaktor bestimmt, mit dem der individuelle Energieumsatz eines jeden Probanden geschätzt wurde. Als Grundlage für diese Berechnung diente der Ruheumsatz des einzelnen Probanden, der mittels der Mifflin-Formel berechnet wurde (vgl. Kapitel 3). 
Die Ergebnisse der Aktivitätsprotokolle ergeben einen durchschnittlichen täglichen Energieumsatz von $3982 \mathrm{kcal} / \mathrm{d}$. Der höchste Wert liegt in t2 im Frühjahr mit 4118 kcal/ d vor. In t1 im Winter wurde mit $3887 \mathrm{kcal} / \mathrm{d}$ der geringste Wert und in t3 im Sommer wurde ein Energieumsatz von $3942 \mathrm{kcal} / \mathrm{d}$ erhoben. Diese Verteilung zeigt sich ebenso bei der Betrachtung der Energieumsätze an Werktagen (Montag bis Donnerstag) und am Wochenende. Dabei ist besonders der niedrige Energieumsatz in $\mathrm{t} 1$ an Werktagen mit $3480 \mathrm{kcal} / \mathrm{d}$ auffällig. Im Vergleich zu t2 mit 4609 kcal/ d ergibt sich eine Differenz von 1129 kcal/ d. Die Differenzen des Energieumsatzes während der beruflichen Tätigkeit stimmen mit diesem Ergebnis überein, da in $\mathrm{t} 1 \mathrm{der}$ Wert von $2581 \mathrm{kcal} / \mathrm{d}$ deutlich unter dem Wert in t 2 mit $2929 \mathrm{kcal} / \mathrm{d}$ liegt. In t3 liegt ein Energieumsatz in beruflichen Tätigkeiten von $2673 \mathrm{kcal} / \mathrm{d}$ vor. Diese Werte, die aufgrund der Erhebungsmethode als Schätzungen zu betrachten sind, sagen aus, dass im Winter bei der Holzernte der geringste Energieumsatz erhoben wurde. Bei der Ausführung von Holzernte, Wegepflege und Jungwuchspflege im Sommer ist der Energieumsatz etwas höher. Der höchste Energieumsatz wird allerdings im Frühjahr bei Pflanzungen und Sammelhieb erreicht. Diese Werte lassen trotz der Tatsache, dass es sich um Schätzwerte handelt, die Aussage zu, dass bei einem Arbeitstag mit Pflanzungen und Sammelhieb im Frühjahr ein höherer Energieumsatz stattfindet als bei einem Arbeitstag mit Holzernte im Winter. Auch im Sommer bei höheren Temperaturen und Tätigkeiten wie Wegepflege, Jungwuchspflege und Holzernte liegt der Energieumsatz unter dem Energieumsatz im Frühjahr. Der hohe Energieumsatz bei Pflanzungen lässt sich zum einen auf das zu tragende Material zurückführen. Die Forstwirte tragen die Jungpflanzen in größeren Mengen während des Pflanzvorganges mit sich. Zum anderen nehmen die Forstwirte eine andauernde gebückte Haltung ein, die seltener durch ein Aufrichten unterbrochen wird als bei anderen forstwirtschaftlichen Tätigkeiten. Das Setzen des Pflanzlochs erfordert bei Bodenbewuchs eine zusätzliche Anstrengung, so dass die unökonomische Haltung und Bewegung und das Tragen, die Monotonie der Pflanzvorgänge und das Tragen der schweren Pflanzen als Ursache für den erhöhten Energieumsatz genannt werden können. Neben dieser Erkenntnis ist zusätzlich zu nennen, dass an Werktagen der größte Teil des Energieumsatzes durch die berufliche Tätigkeit erfolgt, was die hohe körperliche Beanspruchung des Berufsbildes Forstwirt unterstreicht. Bemerkenswert sind die individuellen Ausprägungen des Energieumsatzes, die durch die Minimum- und Maximumwerte zu erkennen sind. Zum einen sind diese großen Unterschiede im Energieverbrauch auf die z. T. unterschiedlichen Tätigkeiten zurückzuführen. Zum anderen begründet sich diese Diskrepanz in personalen Gründen wie Alter, Gewicht und Trainingszustand der Probanden, wobei sich die- 
ser auch zu einem großen Teil durch erlernte motorische Stereotype definiert, die einen ökonomischen Arbeitsablauf ermöglichen.

Die Werte des in der Freizeit stattfindenden Energieumsatzes ähneln sich in allen drei Messzeitpunkten. Ein deutlicher Unterschied ist jedoch zwischen dem freizeitlichen Energieumsatz an Werktagen und am Wochenende festzustellen. Der Energieumsatz am Wochenende mit $2152 \mathrm{kcal}$ ist in allen drei Messzeitpunkten etwa doppelt so groß wie der Energieumsatz an Werktagen mit 1005 kcal. Diese erhöhte Freizeitaktivität am Wochenende lässt sich auf die Zeit zurückführen, die aufgrund der fehlenden beruflichen Tätigkeit am Wochenende vermehrt für freizeitliche Aktivitäten zur Verfügung steht.

Sportliche Aktivitäten erreichen einen maximalen Mittelwert in t2 mit $208 \mathrm{kcal} / \mathrm{d}$. Dieser Mehrverbrauch knüpft an die Ergebnisse an, dass eine höhere körperliche Aktivität im Frühjahr im Vergleich zum Winter festgestellt werden konnte (Bitar et al. 1999; Santos et al. 2005; Ma et al. 2006). An Werktagen wird in $t 1$ der höchste Mittelwert mit 248 kcal, am Wochenende in t 2 mit $257 \mathrm{kcal}$ erreicht. Am Wochenende liegt in $\mathrm{t} 1 \mathrm{der}$ niedrigste Wert mit $23 \mathrm{kcal}$ vor, in $\mathrm{t} 2$ der höchste Wert mit $257 \mathrm{kcal}$ und in $\mathrm{t} 3$ wurde ein Energieumsatz bei sportlicher Aktivität von $156 \mathrm{kcal}$ erhoben. Auch diese Ergebnisse bestätigen eine erhöhte körperliche Aktivität während des Frühjahrs.

Auffällig ist, dass sich die sportliche Aktivität meist auf einzelne Forstwirte verteilt und andere Forstwirte in allen Messzeitpunkten keiner sportlichen Aktivität nachgehen. Diskussionswürdig ist in diesem Zusammenhang, ob eine hohe körperliche Aktivität im Beruf der empfohlenen körperlichen Aktivität in der einschlägigen Literatur entspricht. Häufig wird von positiven Effekten körperlicher Aktivität auf die physische und psychische Gesundheit gesprochen (Hänsel 2007; Schlicht \& Brand 2007, Wagner \& Brehm 2008). Grundlage dieser Aussage bildet die Tatsache, dass in der heutigen Gesellschaft bei beruflichen Tätigkeiten die körperliche Beanspruchung durch meist sitzende Tätigkeiten sehr gering ausfällt. Bei Berufen mit hoher körperlicher Beanspruchung ist es dennoch wichtig, in der Freizeit körperlich aktiv zu sein, um einen Ausgleich auf psychischer als auch auf physischer Ebene zu schaffen (Ilmarinen 1978). Dies kann auch auf die berufliche Situation der Forstwirte übertragen werden. Durch die Monotonie der beruflichen körperlichen Aktivität mit häufigen belastenden Fehlhaltungen ist regelmäßige körperliche Aktivität in der Freizeit notwendig, um präventiv zu handeln (Lippke \& Vögele 2006; Biddle \& Mutrie 2008; Rudolph 2013). Zumal laut Pronk et al. (2004) durch eine höhere körperliche Freizeitaktivität die Arbeitsqualität ansteigt.

Insgesamt kann festgehalten werden, dass sich die Tätigkeit der Forstwirte mit einem durchschnittlichen Energieumsatz an Werktagen von $4157 \mathrm{kcal} / \mathrm{d}$ laut Hollmann \& Hettinger 
(2000, vgl. Abbildung 1) im Übergangsbereich von schwerer zu schwerster Arbeit einordnen lässt. Dies bestätigt die Aussage, dass man bei den in der Studie durchgeführten Arbeitsprozessen von Tätigkeiten mit hoher körperlicher Beanspruchung sprechen kann.

\section{5 Äußere Bedingungen}

Die deskriptive Beschreibung der äußeren Bedingungen bildet die unterschiedlichen und ständig wechselnden Beanspruchungen des Berufsbildes Forstwirt ab. Aufgrund der stark variierenden äußeren Bedingungen wie z. B. Hangneigung, Bodenbewuchs oder meteorologische Einflüsse, ist eine exakte Definition bzw. Standardisierung der einzelnen Tätigkeiten nicht möglich. Aus diesem Grund bleibt bei der Betrachtung der Ergebnisse stets zu berücksichtigen, dass es sich nicht um standardisierte Bedingungen bei den Messungen handelt, so dass ein Vergleich nur bedingt möglich ist.

Diese Tatsache sollte auch bei der Betrachtung des Zusammenhangs zwischen äußeren Bedingungen und relativem Energieumsatz bzw. Sauerstoffverbrauch berücksichtigt werden. Zwischen der Temperatur und dem relativen Energieumsatz konnte kein Zusammenhang festgestellt werden.

Die Hangneigung zeigt nur in $\mathrm{t} 2$ einen positiven Effekt, so dass geschlussfolgert werden kann, dass bei Pflanzungen, die größtenteils in $\mathrm{t} 2$ durchgeführt wurden, eine stärkere Hangneigung zu einem erhöhten relativen Energieumsatz führt. Bei der Holzernte in t1 und bei der Jungwuchspflege und der Holzernte in $\mathrm{t} 3$ zeigt die Hangneigung keinen signifikanten Zusammenhang. Dies könnte daran liegen, dass die Forstwirte bei den Pflanzungen kontinuierlich den Hang hinauf gehen und bei jeder zu setzenden Pflanze die Hangneigung überwunden werden muss. Hingegen wird bei der Holzernte erst nach dem Fällvorgang bei Beginn der Entastung am Hang gelaufen.

Eine positive Korrelation liegt bei der Betrachtung der Stärke der Behinderung in $\mathrm{t} 2$ und in $\mathrm{t} 3$ vor. Der mit der Stärke der Behinderung ansteigende relative Energieumsatz lässt sich in t2 bei den Pflanzungen dadurch erklären, dass die Forstwirte zum einen für jedes Pflanzloch den Boden vom Bewuchs befreien müssen und dass sie sich zum anderen durch den Bewuchs fortbewegen müssen, um die Pflanzlöcher zu setzen. Das zweite Argument erklärt ebenfalls den erhöhten Energieumsatz in $\mathrm{t} 3$ bei der Jungwuchspflege.

Trotz der festgestellten Korrelationen sollte an dieser Stelle bedacht werden, dass es sich um eine geringe Probandenzahl handelt und dass die unterschiedlichen gemessenen Tätigkeiten die Ergebnisse verzerren können. Um exaktere Aussagen zu dem Einfluss der äußeren Bedingungen auf den Energieumsatz machen zu können, müssten mehrere Probanden bei der selben 
Tätigkeit unter variierenden äußeren Bedingungen gemessen werden, um somit sowohl einen intrapersonalen als auch interpersonellen Vergleich zu ermöglichen.

\subsection{Indirekte Kalorimetrie}

In den folgenden beiden Abschnitten werden die Ergebnisse der spiroergometrischen Untersuchung diskutiert und in den Forschungsstand eingegliedert. Nachdem zunächst die Ergebnisse der spiroergometrischen Untersuchung bei den beruflichen Tätigkeiten der Forstwirte eingegangen werden, folgt die Diskussion der Ergebnisse der Fahrradspiroergo-metrie und deren Verknüpfung miteinander.

\subsubsection{Spiroergometrie}

$\mathrm{Zu}$ Beginn dieses Kapitels wird auf die Werte der jeweiligen Messzeitpunkte eingegangen, sowohl auf die Mittelwerte als auch auf die Werte einzelner Probanden. Anschließend werden die nach den Tätigkeiten der Forstwirte kategorisierten Werte und die gemessenen und errechneten Grund- und Ruheenergieumsätze diskutiert.

Die Sauerstoffaufnahme bei der Holzernte in t1 weist mit 2,04 1/ min den höchsten Wert auf. Dies bestätigt, dass die motormanuelle Holzernte die Tätigkeit darstellt, die körperlich am anspruchvollsten für die Forstwirte ist bzw. einen hohen Energieumsatz beansprucht. Auch die Werte im Frühjahr und im Sommer weisen eine hohe Sauerstoffaufnahme auf, liegen jedoch mit 1,76 1/ min und 1,63 1/ min unter den Werten, die im Winter gemessen wurden. Die hohen Standardabweichungen besonders in $\mathrm{t} 2$ weisen jedoch auf interpersonale Varianzen hin. Diese großen Differenzen lassen sich dadurch erklären, dass die Forstwirte in $\mathrm{t} 2$ und $\mathrm{t} 3$ unterschiedliche Tätigkeiten ausgeführt haben. Darüber hinaus verfügen die Forstwirte aufgrund ihrer unterschiedlichen Berufserfahrung über unterschiedliche motorische Stereotype. Durch langjährige Berufserfahrung und ausgebildete Bewegungsmuster können die Bewegungen ökonomischer ausgeführt werden. Des Weiteren haben die Probanden unter unterschiedlichen äußeren Bedingungen wie Hangneigung, Wetter, Temperatur und Bodenbewuchs gearbeitet, so dass diese durchaus einen Einfluss auf den Energieumsatz ausüben können. Die relativ konstanten Werte bei FW08 lassen vermuten, dass der Proband zu allen Messzeitpunkten und somit bei den ausgeführten motormanuellen Tätigkeiten (Holzernte, Sammelhieb) eine ähnliche körperliche Beanspruchung aufweist.

Bei der Betrachtung des relativen Energieumsatzes fällt auf, dass hier ebenfalls der höchste Wert in 11 gemessen wurde. Aufgrund der Abhängigkeit vom im Verlauf des Erhebungszeitraums angestiegenen Körpergewichts relativieren sich jedoch einige Werte. Die intrapersonalen Schwankungen sind mit den hohen Werten in t1 (FW02, FW06, FW14) mit der hohen 
Beanspruchung der Holzernte zu erklären. Einzelne niedrige Werte sind z. T. auf die geringe Beanspruchung der Tätigkeit zurück zu führen. FW09 und FW10 führten z. B. in t2 Arbeiten mit dem Pflanzlochbohrer aus, bei denen zwar eine belastende Körperhaltung eingenommen wird, die Haupttätigkeit jedoch in der Führung der Maschine liegt, welche das Pflanzloch bohrt.

Die RQ-Werte liegen zwischen 0,84 und 0,91 und sprechen für eine Energiegewinnung, die hauptsächlich durch die Oxidation von Proteinen und Kohlenhydraten stattfindet. Drei Forstwirte liegen in jeweils einem Messzeitpunkt über 1,0. Dies könnte seine Ursache in einer Hyperventilation, in der Intensität der Belastung, in einer Kohlenhydratmast oder auch mit dem Zeitpunkt der letzten Nahrungsaufnahme haben. Die Ursache lässt sich jedoch nicht konkret benennen. Der recht hohe RQ-Wert lässt sich jedoch mit der Aussage in Verbindung bringen, dass körperlich Aktive zu 60 \% ihre Energie durch die Kohlenhydratoxidation gewinnen.

Die Signifikanzen im Paarvergleich unterstreichen die Aussage, dass besonders die Holzernte in $\mathrm{t} 1$ eine hohe Beanspruchung im Sinne eines erhöhten Energieumsatzes darstellt. Dies unterstützt die These von Rudolph (2013), indem hiermit die unterschiedliche motorische Beanspruchung zu den einzelnen Jahreszeiten durch die unterschiedliche Höhe des Energieumsatzes erweitert wird. Jedoch muss hier eine differenzielle Betrachtung von motorischer Beanspruchung im Sinne der Belastung der muskuloskelettalen Strukturen und des Energieumsatzes gemacht werden, da diese nicht gleich zu setzen sind.

Eine genauere Betrachtung der einzelnen Tätigkeiten führt zu zwei Aussagen. Zum einen ist die Holzernte die häufigste gemessene Tätigkeit, die sowohl in $\mathrm{t} 1$ als auch in $\mathrm{t} 3$ durchgeführt wurde. Dies passt zu der Aussage, dass in den letzten Jahren die Holzernte vermehrt den Hauptaufgabenbereich der Forstwirte umfasst.

Zum anderen ergab eine Berechnung des MET, dass die Tätigkeiten der Forstwirte mit einem MET zwischen 5 und 7 definiert werden können. Obwohl ausgesagt wird, dass bei schwerer körperlicher Arbeit Energieumsätze des 20fachen des Grundumsatzes erreicht werden können, sind diese Multiplikationsfaktoren sehr hoch und in den Bereich über der Schwerstarbeit einzuordnen, der bei 3 MET liegt. Auch die Sauerstoffaufnahme liegt mit Werten zwischen 1,3 1/ min und 1,9 1/ min deutlich über den von Persson (2007) aufgestellten zulässigen Höchstwerten für jahrelange berufliche Arbeit, die bei einem $88 \mathrm{~kg}$ schweren Mann 0,867 1/ min entspricht. Diese hohen Werte spiegeln das hohe Anforderungsprofil der Forstwirte wider. Bei allen gemessenen Tätigkeiten werden Werte erreicht, die über dem Fünffachen des Grundumsatzes liegen und somit einer körperlich sehr schweren Arbeit entsprechen. Für diese hohen Werte gibt es zwei Erklärungsmöglichkeiten. Zum einen muss berücksichtigt werden, 
dass es sich um Werte handelt, die während der Reinen Arbeitszeit gemessen wurden. Verteilund Rüstzeiten finden bei dieser Messung keine Berücksichtigung und lassen den Wert bei der Betrachtung eines kompletten Arbeitstages sinken. Zum anderen wurden die Messungen zur Hochsaison der jeweiligen Tätigkeiten durchgeführt. Die sehr hohen Energieumsätze lassen darauf schließen, dass die Forstwirte zeitweise diesen hohen Beanspruchungen ausgesetzt sind, jedoch Phasen mit einem niedrigeren Energieumsatz, in denen wetterbedingt oder aus organisatorischen Gründen die Tätigkeit nicht durchgeführt werden kann, diese hohen Energieumsätze ausgleichen. Somit sind die Forstwirte phasenweise negativen Energiebilanzen ausgesetzt, welche dann mit weniger arbeitsintensiven Phasen und vermutlich positiven Energiebilanzen zu einer Balance im Energiehaushalt führen.

Darüber hinaus sollte berücksichtigt werden, dass keine Standardisierung der Tätigkeiten erfolgen kann, da die Arbeit der Forstwirte durch diverse aufgeführte Einflüsse variiert. Dies wird auch bei der Betrachtung der Werte bereits durchgeführter Studien bestätigt, die Differenzen beim Energieumsatz angeben. Im Vergleich zu den bisher erhobenen Daten fällt auf, dass die Werte der vorliegenden Studie relativ hoch sind. Gründe hierfür wurden bereits diskutiert (s. o.). Ergänzend lässt sich festhalten, dass die Differenzen evtl. auf die unterschiedliche Datierung der Studien zurückzuführen sind. Außerdem zeigen die Differenzen in den einzelnen Studien auf, dass bei der Ausführung der Tätigkeiten eine hohe Variabilität vorliegt, die sich u. a. in unterschiedlich hohen Energieumsätzen äußert. Und auch wenn z. B. beim Wegebau ein geringerer Energieumsatz vorliegt, bleibt bei der Arbeitsplatzanalyse dennoch $\mathrm{zu}$ beachten, dass hier ebenso eine belastende Körperhaltung mit vermehrter statischer Muskelaktivität über einen längeren Zeitraum eingenommen wird.

Abschließend werden die unterschiedlichen Ruheenergie- und Grundumsätze diskutiert. Für die Auswertung der Energieumsätze dient der Grundumsatz als Basis. Da dieser aus organisatorischen Gründen nicht gemessen werden konnte, wurde er mit der Mifflin-Formel berechnet (1,26 kcal/ min), die in der Literatur als die exakteste tituliert wird (vgl. Kapitel 3). Die Abweichung vom mit dem KÄ errechneten Grundumsatz (1,41 kcal/ min) ist diskussionswürdig. Jedoch lässt sich konstatieren, dass sich die Werte der Mifflin-Formel eher dem Richtwert von $1 \mathrm{kcal} / \mathrm{min}$ nähern. Dass die Grundumsätze über dem Richtwert liegen, liegt am Körpergewicht der Forstwirte und an der vermehrten Muskelmasse, die durch die körperlich anstrengende Arbeit entsteht und für eine Erhöhung des Grundumsatzes sorgt. Die hohen Werte der Ruheenergieumsatzmessung mittels Spirometrie lassen sich ebenfalls durch eine erhöhte Muskelmasse, gesteigerte regenerative Prozesse und fehlende körperliche Inaktivität und 
Nahrungskarenz vor der Messung begründen. Zusätzlich führen die ungewohnte Situation, die Aufregung und die fehlende Gewöhnung zu einem erhöhten Ruheenergieumsatz.

\subsubsection{Fahrradspiroergometrie}

Die Fahrradspiroergometrie dient dazu, die erbrachte Leistung, die bei der nicht standardisierbaren Messung im Feld gemessen wurde, in physikalischen Maßen ausdrücken zu können. Dabei findet eine Zuordnung zu den Wattstufen statt.

Für die Wahl der Wattstufen wurde der Energieumsatz bei der im Feld gemessenen Tätigkeit als Richtwert genommen, um bei der Fahrradspiroergometrie einen ähnlichen Energieumsatz zu erreichen und somit die Zuordnung zu ermöglichen. Die Forstwirte führten in allen drei Messzeitpunkten dieselbe Tätigkeit aus, weisen aber einen unterschiedlichen relativen Energieumsatz auf (vgl. Tabelle 15). Dies begründet die unterschiedlichen Leistungen in Watt, bei denen die Forstwirte die Fahrradspiroergometrie durchführen, um diese relativen Energieumsätze zu erreichen. Darüber hinaus wurden für FW01 relativ hohe Leistungen in Watt geschätzt, die während der Fahrradspiroergometrie in t1 festgelegt wurden. Dass FW01 einen unerwartet niedrigen relativen Energieumsatz bei der Fahrradspiroergometrie aufweist, kann u. U. an ausgeprägten motorischen Stereotypen liegen, da der Proband regelmäßig Rad fährt und diese Bewegung und Beanspruchung für ihn bekannt ist und somit ökonomisch ausgeführt werden kann.

Diese interpersonellen Varianzen finden sich ebenfalls in der Zuordnung von Sauerstoffaufnahme und Wattbereich wieder. Den im Feld gemessenen Tätigkeiten kann in t1 eine Leistung von 110 bis $190 \mathrm{~W}$, in $\mathrm{t} 2$ eine Leistung von 120 bis $140 \mathrm{~W}$ und in $\mathrm{t} 3$ eine Leistung von 115 bis $140 \mathrm{~W}$ zugeschrieben werden.

Vergleicht man diese Leistungsbereiche in Watt mit den Angaben von Persson (2007), so lässt sich die Leistung der Forstwirte zwischen dem Energieumsatz in der Freizeit (115 W) und dem zulässigen Höchstwert für berufliche Arbeit $(240 \mathrm{~W})$ einordnen. Somit kann die Leistung der Forstwirte beim Pflanzen, bei der Läuterung und besonders bei der Holzernte als eine hohe Beanspruchung eingeschätzt werden. Bei dieser Einschätzung gilt es zu berücksichtigen, dass es sich um einen unspezifischen Test handelt, da er nicht die motorische Beanspruchung der beruflichen Tätigkeiten verlangt, so dass die zugeordneten Leistungen nur bedingt verallgemeinert und auf die beruflichen Tätigkeiten übertragen werden können.

\subsection{Energiebilanz}

Um die Energiebilanz zu ermitteln, wurde der Energieumsatz von der Energieaufnahme subtrahiert. In allen drei Messzeitpunkten wurden negative Energiebilanzen mit durchschnittlich - 
$6957 \mathrm{kcal}$ pro Woche ermittelt, welche eine Energiebilanz von $-993 \mathrm{kcal}$ pro Tag ergeben. Diese Ergebnisse spiegeln keine Balance zwischen Energieaufnahme und Energieumsatz wider, die in einem 7-Tage-Rhythmus stattfinden sollte (Kasper 2000). Besonders an Arbeitstagen (Montag bis Donnerstag) wird eine negative Energiebilanz deutlich. In t1 wird die höchste Energiebilanz mit $-110 \mathrm{kcal}$ und in $\mathrm{t} 2$ die niedrigste Energiebilanz mit $-425 \mathrm{kcal}$ erreicht. Aufgrund der ähnlich hohen Energieumsätze in allen drei Messzeitpunkten sind die Schwankungen bei der Energiebilanz auf eine erhöhte Energiezufuhr in t1 zurückzuführen. Durch die fehlende berufliche Aktivität am Wochenende wird hier in $\mathrm{t} 2$ die einzige positive Energiebilanz mit $356 \mathrm{kcal}$ erreicht und auch in $\mathrm{t} 1$ und $\mathrm{t} 3$ eine annähernd ausgeglichene Energiebilanz erreicht. Diese Werte lassen darauf schließen, dass die Forstwirte am Wochenende ihre Ernährungsgewohnheiten beibehalten und nicht an die geringere körperliche Aktivität anpassen. Die nahezu an allen Tagen gemessene negative Energiebilanz steht im Kontrast zu der Gewichtszunahme und dem steigenden Körperfettanteil der Probanden. An dieser Stelle wird auf die in Kapitel 9.2 und 9.3 angesprochenen Probleme bei der Erhebung der Ernährungs- und Aktivitätsprotokolle verwiesen. Darüber hinaus ist anzumerken, dass die Probanden stets in einer Zeit die Erhebung durchgeführt haben, in der sie ihrer Tätigkeit nachgehen konnten. Es ist zu bedenken, dass die Forstwirte z. B. bei schlechtem Wetter ihrer Tätigkeit nicht nachkommen können und somit einen geringeren Energieumsatz haben. Bei den Messzeitpunkten handelt es sich um Wochen, in denen die Arbeit kontinuierlich und mit hoher Intensität durchgeführt wurde. Dies ließe in Ansätzen die niedrige Energiebilanz erklären. Zwischen der Energiebilanz und dem Energieumsatz bei der beruflichen Aktivität besteht eine negative Korrelation. Diese ist hauptsächlich dadurch zu erklären, dass die Aktivität eine entscheidende Variable für die Berechnung der Energiebilanz ist.

\section{Diskussion der Untersuchungsmethoden}

Nachdem die Ergebnisse der Untersuchung dargestellt und diskutiert wurden, werden im Folgenden die Erhebungsinstrumente der Studie diskutiert. Dabei wird sowohl auf die qualitativen Methoden als auch auf die Kalipermetrie und die indirekte Kalorimetrie unter Berücksichtigung theoretischer Grundlagen und praktischer Erfahrungen aus der Studie eingegangen.

\subsection{Anthropometrische Daten}

Die Klassifizierung von Adipositas anhand des BMI wird seit Jahren kontrovers diskutiert und führt bei gut trainierten Menschen mit viel Muskelmasse zu einem BMI, der im übergewichtigen oder adipösen Bereich eingeordnet wird. Trotzdem gilt der BMI aus wissenschaftli- 
cher Sicht als international anerkannte Angabe zur Diagnose des Übergewichts (vgl. Kapitel 4.2), weshalb in der vorliegenden Studie der BMI aus der gemessenen Körpergröße und dem gemessenen Körpergewicht errechnet wurde. Bei der Messung wurde in allen drei Messzeitpunkten dieselbe Waage verwendet und die Probanden wurden ohne Arbeitskleidung gewogen. Hierdurch wurden Werte erhoben, die aufgrund der Verwendung der kalibrierten Waage als exakt bezeichnet werden können.

Vor dem Hintergrund eines hohen BMI bei mehr Muskelmasse wurde zusätzlich eine Erhebung des Körperfettanteils durchgeführt. Die Kalipermetrie wurde durch einen geübten Tester an jeweils zehn Körperstellen vorgenommen. Dabei wurde jede Körperstelle drei Mal gemessen, so dass aus diesen drei Werten ein Mittelwert gebildet werden konnte und die Ergebnisse durch diese Mittelung genauer wurden. Der Kaliper wurde zu Beginn der Studie geeicht, so dass gewährleistet werden konnte, dass der Kaliper während der Untersuchungen einen konstanten Druck von $10 \mathrm{~g} / \mathrm{mm}^{2}$ ausgeübt hat. Auch wenn es sich bei der Berechnung des Körperfettanteils um Schätzwerte handelt, bietet die Kalipermetrie eine hohe Praktikabilität im Feld und eine ausreichende Präzision, um anhand der Werte die erhobenen BMI-Werte zu beurteilen. Durch die Erhebung des BMI und des Körperfettanteils wird ein Vergleich der beiden Werte in den drei Messzeitpunkten ermöglicht.

\subsection{Ernährungsprotokoll}

Das 7-Tage-Ernährungsprotokoll als Erhebungsmethode erfüllt verschiedene Kriterien. Zum einen umfasst es den Zeitraum einer ganzen Woche, so dass ein Vergleich zwischen Werktagen und Wochenendtagen möglich ist. Eine Ausweitung der Protokollierung auf einen längeren Zeitraum pro Messzeitpunkt wurde nicht in Betracht gezogen, um den Aufwand für die Probanden möglichst gering zu halten. Sowohl auf die zeitliche Erfassung der Mahlzeiten und die Beschreibung, in welchem sozialen Umfeld die Mahlzeiten eingenommen wurden, könnte im Nachhinein verzichtet werden. Eine ausführliche Analyse dieser Parameter ist nicht im Sinne der Fragestellung und würde den Rahmen dieser Arbeit übersteigen. Zwar kann aus den erhobenen Angaben geschlussfolgert werden, dass eine regelmäßige Einnahme von Mahlzeiten aufgrund der Struktur des Arbeitstages stattfindet und dass das soziale Umfeld eine Auswirkung auf die Energieaufnahme hat, jedoch steht der Aufwand der Erhebung mit dem Nutzen für die Zielsetzung der Arbeit nicht im Verhältnis. Besonders wenn man die Stimmigkeit zur Zielgruppe überprüft, ist festzuhalten, dass eine Berufsgruppe, die fast ausschließlich körperliche Arbeit verrichtet, mit einem sehr hohen schriftlichen Arbeitsaufwand zusätzlich zur Arbeitszeit konfrontiert wird. An dieser Stelle ist ebenfalls hinzuzufügen, dass der Text zur 
Erklärung des Protokolls bewusst kurz und einfach formuliert wurde. Eine wiederholte präzise Erwähnung, dass ein genaues und ehrliches Protokollieren für die Erhebung notwendig ist, hätte vor jedem Messzeitpunkt wiederholt werden müssen, um die Wichtigkeit zu unterstreichen.

Das Schätzen der Mengen wurde aus praktikablen Gründen gewählt und hat sich bewährt. Einige Forstwirte haben z. T. die Mengen gewogen, so dass hier eine exaktere Protokollierung vermutet werden kann. Durch die Anleitung und die exemplarischen Mengenangaben in der Anleitung wurde die Nahrungsaufnahme ausreichend exakt und so genau wie möglich geschätzt. Auch wenn die Erhebung der Menge kritisch betrachtet werden kann, so wurde bei der Zusammensetzung der Nahrung durch das 7-Tage-Protokoll eine recht genaue Erhebung gewährleistet.

Problematisch sind neben den ungenauen Mengenangaben eventuelle Fehlprotokollierungen. Ursache hierfür kann zum einen sein, dass bestimmte Angaben bewusst nicht protokolliert wurden. Zum anderen kann evtl. für die Zeit der Erhebung auf bestimmte Nahrungsmittel und Getränke verzichtet worden sein, um diese nicht im Protokoll aufführen zu müssen.

Neben einer intensiveren Aufklärung der Probanden hat sich das Ernährungsprotokoll als zielführend erwiesen. Aufgrund einiger Kriterien, die bei einem solchen Protokoll nicht vollkommen erfüllt werden können, wurde von vornherein bei der Auswertung der Ergebnisse berücksichtigt, dass es sich um Schätzwerte handelt, die entsprechend interpretiert werden.

\subsection{Aktivitätsprotokoll}

Die Erhebung der Aktivitäten erfolgte in Anlehnung an die Ernährungserhebungsmethode mittels eines Aktivitätsprotokolls. Der Erhebungszeitraum umfasst zu allen drei Messzeitpunkten sieben Tage, um die unterschiedlichen Werte von Werktagen und Wochenende miteinander vergleichen zu können. Der gewählte Zeitraum von einer Woche reicht aus, um eine Balance im Energiehaushalt zu erfassen (Kasper 2000). Aktivitätsprotokolle ermöglichen eine parallele Erhebung mehrerer Probanden, so dass eine Protokollierung zum gleichen Zeitpunkt möglich war. Die Protokollierung erfordert wie die Ernährungsprotokollierung eine gute Kooperation von Seiten der Probanden. Durch die Form einer Kreuztabelle wird den Probanden das Ausfüllen erleichtert und die Protokolle werden für alle drei Messzeitpunkte standardisiert. Darüber hinaus werden die Tätigkeiten in allen Bereichen möglichst exakt mit Beispielen beschrieben. Die Aktivitäten während der Berufstätigkeit wurden an das Anforderungsprofil der Forstwirte adaptiert, so dass das Ankreuzen und Zuordnen der körperlichen Aktivität erleichtert wurde. 
Mit der Formel von Mifflin (1990) wurde als Grundlage für das Aktivitätsprotokoll der Grundumsatz der Probanden berechnet, der einen Mittelwert von 1,26 kcal/min ergibt. Im Vergleich zu dem mit dem KÄ errechneten Grundumsatz und zum gemessenen Grundumsatz ist der Wert nach Mifflin niedriger. Eine ausführliche Diskussion der Problematik findet sich in Kapitel 9.5.1. Der Grundumsatz nach der Mifflin-Formel wird nichtsdestotrotz als Grundlage für die Berechnung des Energieumsatzes verwendet, da sich die Formel u. a. in einer Studie von Frankenfield et al. (2003) als die exakteste erwies. Dieser individuelle Wert entspricht dem Multiplikationsfaktor 1. Für alle anderen körperlichen Tätigkeiten wurden in Anlehnung an die Studie von Ainsworth (2011) Multiplikationsfaktoren festgelegt, die von Ainsworth aus diversen Studien zusammengefügt wurden, mittels derer der Energieumsatz berechnet wurde. Bei den Multiplikationsfaktoren handelt es sich um Schätzwerte, so dass dieser Aspekt auch bei der Interpretation des Energieumsatzes Berücksichtigung finden muss. Da bisher keine entsprechende Methode alle Aspekte körperlicher Aktivität berücksichtigt (Schutz et al. 2001; van Hees et al. 2013) und es sich bei der Berechnung mit Hilfe des Aktivitätsprotokolls und der Multiplikationsfaktoren lediglich um Schätzwerte handelt, wurde das Aktivitätsprotokoll durch die indirekte Kalorimetrie ergänzt. Durch diese Kombination konnten tatsächlich gemessene Werte für die berufliche Haupttätigkeit während des jeweiligen Erhebungszeitraumes ermittelt werden. Das Aktivitätsprotokoll wird in diesem Punkt somit um einen exakten individuellen Wert ergänzt. Die gemessenen Energieumsätze wurden nur prozentual in das Aktivitätsprotokoll eingepflegt, da auftretende Erholungs-, Verteil- und Rüstzeiten bei der Messung nicht erfasst wurden und aus diesem Grund nachträglich berücksichtigt wurden (vgl. Kapitel 6.3.3). Der Tatsache, dass die indirekte Kalorimetrie aus zeitlichen, finanziellen und für die Probanden belastenden Gründen nicht den gesamten Arbeitstag durchgeführt werden konnte, wurde hiermit begegnet. Der gemessene Energieumsatz wurde somit an die Arbeitsabläufe und Strukturen der Arbeitstage angepasst.

Die anzukreuzenden Zeitintervalle umfassen 15 Minuten. Dieses gewählte Intervall ist bei kurz andauernden Tätigkeiten etwas ungenau, ist jedoch im Hinblick auf die Praktikabilität des Protokollierens als realistisch einzuschätzen. Es entsteht sowohl bei der Protokollierung als auch bei der Erfassung der Daten ein recht hoher Aufwand, dem mit dem Verfahren des Ankreuzens zwar entgegengewirkt wird, aber sich nicht weiter reduzieren lässt, wenn eine möglichst exakte Erfassung der körperlichen Aktivität erzielt werden soll. Umso bedeutender war es, die Probanden zu instruieren und ihnen die Notwendigkeit exakter und ehrlicher Angaben zu verdeutlichen. Für die Fragestellung der vorliegenden Arbeit nach der Energiebilanz und nach dem Energieumsatz zu unterschiedlichen Jahreszeiten ist die Einteilung des Aktivi- 
tätsprotokolls in körperliche Aktivität während der Berufstätigkeit und während der Freizeit notwendig. Anhand dieser Unterteilung konnte ein Vergleich des gesamten Energieumsatzes, des Energieumsatzes bei beruflicher Tätigkeit und des Energieumsatzes bei Freizeitaktivität in den drei Messzeitpunkten vorgenommen werden.

\section{4 Äußere Bedingungen}

Unter äußeren Bedingungen werden in dieser Arbeit die Gelände- und Witterungsverhältnisse als auch die Temperaturen verstanden, wobei die Geländeverhältnisse in Hangneigung und Bewuchs unterteilt werden. Für die Erhebung wurden Kategorien gebildet, um den Forstwirten das Protokollieren zu erleichtern. Diese Einteilung in unterschiedliche Bereiche richtet sich nach in der Forstwissenschaft gängigen Angaben, so dass diese für die Probanden verständlich und nachvollziehbar sind. Die zeitliche Einteilung ist auf den Protokollen offen gelassen, so dass die Forstwirte bei einem Wechsel der Bedingungen dies entsprechend eintragen können. Auf eine kleinschrittigere Erhebung (z. B. stündlich) wurde verzichtet, um den Aufwand gemessen am Nutzen der Ergebnisse vertretbar zu halten. Bei der Auswertung hat sich herausgestellt, dass eine Unterteilung in Vormittags- und Nachmittagswerte ausreicht, da sich die Bedingungen nicht verändert haben oder da die Probanden nur in größeren Zeitintervallen die äußeren Bedingungen angegeben haben.

Hinsichtlich der Untersuchungsmethodik sind daher zwei Aspekte kritisch zu diskutieren. Zum einen wäre für eine exakte Protokollierung eine Angabe in festgelegten Zeitintervallen notwendig gewesen. Diese hätte an das Layout der Aktivitätsprotokollierung angepasst werden können, um durch die einheitliche Gestaltung die Protokollierung zu vereinfachen. Hieraus ergibt sich der zweite Aspekt, der sich auf die Relevanz der äußeren Bedingung für die Frage nach der Energiebilanz bezieht und somit auf den Aufwand-Nutzen-Faktor. Die äußeren Bedingungen sollten aufgrund des Einflusses auf den Energieumsatz erhoben werden. Um diesen Einfluss in der Erhebung des Energieumsatzes und somit in der Auswertung der Aktivitätsprotokolle zu berücksichtigen, hätten Kennwerte über die Höhe dieses Einflusses vorliegen oder erhoben werden müssen. In der Literatur werden keine konkreten Aussagen hierzu gemacht, es wird lediglich festgestellt, dass diese Einflüsse vorliegen. Da die mittels Spiroergometrie gemessenen Werte zum Energieumsatz bei beruflicher Tätigkeit sehr variieren und da es sich lediglich um 14 Probanden handelt, ist eine Schlussfolgerung auf die Höhe des Einflusses der Bedingungen nicht möglich. Für eine exakte Erhebung wäre eine spiroergometrische Messung bei den jeweiligen Tätigkeiten bei jedem einzelnen Probanden unter verschiedenen äußeren Bedingungen notwendig gewesen. Auf diese Weise hätten Werte erhoben wer- 
den können, die einerseits bei der Auswertung der Aktivitätsprotokolle hätten berücksichtigt werden können und andererseits eine Tendenz über das Ausmaß der verschiedenen Einflussfaktoren auf den Energieumsatz aufgewiesen hätten. Dieses methodische Vorgehen wird letztendlich durch zeitliche, organisatorische und finanzielle Gründe limitiert.

Aus den aufgezeigten Gründen lassen sich aus den protokollierten Ergebnissen nur Rückschlüsse ziehen, unter welchen Bedingungen die Forstwirte im Messzeitraum gearbeitet haben. Diese Bedingungen können bestenfalls in die Diskussion der Energieumsätze einfließen. Ein direkter Zusammenhang zwischen äußeren Bedingungen und Energieumsatz kann zumindest punktuell bei der Auswertung der Spiroergometrie untersucht werden. Durch die Betrachtung von Energieumsatz und einzelnen äußeren Bedingungen kann eine aspezifische Aussage zum Zusammenhang dieser beiden Faktoren gemacht werden. Aufgrund der multifaktoriellen Einflüsse und aufgrund der hohen Individualität der Energieumsätze zeigen aber auch diese Ergebnisse nur eine Tendenz auf.

\subsection{Indirekte Kalorimetrie}

Die indirekte Kalorimetrie erfolgte über die Bestimmung der Sauerstoffaufnahme mittels Spiroergometrie mit dem Gerät MetaMax3B der Firma Cortex. Während der beruflichen Haupttätigkeiten der Probanden wurde die Messung mit einer mobilen Messeinheit durchgeführt, die mit einem angepassten Gesichtsschutz bei allen Forstarbeiten getragen werden konnte. Durch das geringe Gewicht des Gerätes und dessen Fixierung, die die Bewegungsfreiheit der Forstwirte nicht einschränkt, hat sich der Einsatz des Gerätes bei der Forstarbeit bewährt und die Praktikabilität bei Felduntersuchungen bestätigt.

Im Hinblick auf die Messung der $\mathrm{VO}_{2}$-Werte diskutieren verschiedene Autoren (vgl. Kapitel 6.3.5) eine nicht ausreichende Validität. Aufgrund der dieser Diskussion zugrunde liegenden Studien ist eine exakte Messung in der vorliegenden Untersuchung nicht gewährleistet. Um auf diesen Aspekt zu reagieren, wurde eine regelmäßige Kalibrierung des Gerätes zur Sicherstellung der Exaktheit der Messung durchgeführt. Nichts desto trotz haben bei der Diskussion der Ergebnisse diese Kritikpunkte Berücksichtigung gefunden.

\subsubsection{Spiroergometrie}

Für eine möglichst standardisierte und objektive Diagnostik des Energiestoffwechsels wurde die in Studien am häufigsten genutzte Spiroergometrie angewendet. Während alltäglicher körperlicher Belastungen wird der Energieumsatz mittels physiologischer Parameter ermittelt. Dabei wurde vor dem Hintergrund der Fragestellung der vorliegenden Arbeit ein besonderer 
Fokus auf den Sauerstoffverbrauch, die Kohlendioxidproduktion und den respiratorischen Quotienten gelegt.

Bei der Anwendung in der Praxis kam es auf eine gute Fixierung des Gurtes an, um das Tragen des Gurtes zu sichern und trotzdem die normalen Arbeitsabläufe und Bewegungen der Probanden zu ermöglichen bzw. nicht zu behindern. Bei leichtem Regen wurde das Spirometer unter der Regenkleidung getragen, so dass auch hier eine Messung möglich war. Bei starkem Regen können die Forstwirte ihre Haupttätigkeiten nicht ausführen, so dass eine Messung dann nicht notwendig war. Grundsätzlich lässt sich festhalten, dass durch die flexible Anwendung eine Messung zu allen drei Messzeitpunkten und somit bei allen unterschiedlichen Tätigkeiten möglich war.

Wie bereits vermutet (vgl. Kapitel 6.3.5.1), war aufgrund des Arbeitsablaufes eine Messung des Grund- oder Ruheumsatzes nicht möglich. Dennoch nahmen die Forstwirte während der Kalibrierung der Gassensoren und während der ersten drei Messminuten eine sitzende Position ein. So wurde besonders bei der ersten Messung versucht, die Probanden mit der ungewohnten Situation vertraut zu machen und ihnen die Möglichkeit zu geben, sich an das Tragen der Atemmaske und das Atmen zu adaptieren. Die meisten Probanden sagten aus, dass das Tragen des Gerätes und der Gesichtsmaske ihre Arbeitsabläufe nicht eingeschränkt haben und die Atmung nicht erschwert wurde. Dennoch sollten die emotionale und psychische Belastung bei einer solchen Erhebung hinsichtlich der Diskussion der Ergebnisse berücksichtigt werden.

Die gemessenen Werte für den Sauerstoffverbrauch bei der Ausführung der beruflichen Tätigkeit sind relativ hoch, weshalb bei deren Berücksichtigung im Aktivitätsprotokoll eine prozentuale Berechnung gemäß der Reinen Arbeitszeit und der Rüstzeit vorgenommen wurde (vgl. Kapitel 9.3). Ein weiteres Problem bei der Messung der beruflichen Haupttätigkeit stellte zum einen die Tatsache dar, dass die Forstwirte nicht alle in den Messzeitpunkten derselben Tätigkeit nachgehen. Lediglich in $\mathrm{t} 1$ führen alle Forstwirte Holzernte aus. In den anderen Messzeitpunkten variieren die Tätigkeiten, so dass ein direkter Vergleich problematisch ist. Zum anderen gestaltet sich auch bei derselben Tätigkeit die Ausführung sehr individuell. Beeinflussende Faktoren sind hierbei sowohl personale Voraussetzungen (z. B. Alter, Berufserfahrung, motorische Stereotype) als auch Arbeitsbedingungen (z. B. bei der Holzernte die Größe der Krone beim Entasten, das Keilen oder bei Pflanzungen das Pflanzverfahren oder die Bodenbeschaffenheit). Diese Bedingungen erschweren einen Vergleich des Energieumsatzes bei den Tätigkeiten, ermöglichen aber das Abschätzen des Energieumsatzes bei der Aus- 
führung der Tätigkeit über einen Arbeitstag, so dass das Ermitteln der Energiebilanz durch die Werte der Spiroergometrie exakter ist.

\subsubsection{Fahrradspiroergometrie}

Durch die Spiroergometrie bei der beruflichen Tätigkeit wird der Energieumsatz ermittelt, der eine Aussage über die Arbeitsschwere macht. Um die erbrachte Leistung in physikalischen Maßen ausdrücken zu können, wurde bei vier Forstwirten eine Fahrradspiroergometrie durchgeführt. Anhand der hier erhobenen Werte wird eine Zuordnung des Energieumsatzes bei der beruflichen Tätigkeit zu Leistungen in Watt ermöglicht.

Durch die Vorstartsituation, die sitzend auf dem Fahrradergometer gemessen wird, wird ähnlich wie bei der Felduntersuchung die Gewöhnung des Probanden an die Situation ermöglicht. Vor dieser Messung haben die Forstwirte jedoch nicht gearbeitet. Die Forstwirte fahren drei Belastungsstufen à zehn Minuten, die anhand der Werte der erhobenen Daten bei beruflicher Tätigkeit abgeschätzt werden. Dieses Zeitintervall lehnt sich zum einen an die gemessene Zeit im Feld an und ermöglicht zum anderen eine Adaptation der Probanden an die Belastungsstufe. Die vorgegebene Drehzahl von 50 bis 60 Umdrehungen pro Minute, um einen optimalen Wirkungsgrad zu erzielen, konnte von allen Probanden problemlos umgesetzt werden.

Die Fahrradspiroergometrie stellt tatsächlich nur eine Zuordnung des Sauerstoffverbrauchs zur Leistung in Watt dar. Aufgrund der diskutierten Problematik der motorischen Stereotype beim Fahrradfahren (vgl. Kapitel 8.5.2) können die zugeordneten Leistungen in Watt von der tatsächlich erbrachten Leistung bei der beruflichen Tätigkeit differieren. Dennoch bietet die Zuordnung einen Anhaltspunkt für die individuelle Leistung der Forstwirte bei der körperlichen Aktivität im Beruf, so dass eine Messung aller Probanden mittels Fahrradspiroergometrie wünschenswert gewesen wäre, um allen erhobenen Werten Leistungen in Watt zuordnen und diese miteinander vergleichen zu können. 


\section{Fazit und Ausblick}

Bewegungsmangel und Übergewicht durch Fehlernährung bilden in den Industrieländern heutzutage die größten Gesundheitsrisiken. Basierend auf diesem Ist-Zustand entwickelten sich in den letzten Jahren Präventions- und Interventionskonzepte, die sich dieser Risikofaktoren annehmen. Außer Acht gelassen werden bei dieser Schwerpunktsetzung in der Forschung und in der Praxis Berufsgruppen mit einem ausgeprägten körperlichen Anforderungsprofil. Dieser hohen beruflichen Aktivität sind gesundheitlich positive Auswirkungen nicht immanent. Gegenteilig wurde nachgewiesen, dass bewegungsbezogene Interventionen bei körperlich aktiven Arbeitern notwendig sind, um zum einen psychosoziale Aspekte aufzugreifen und zum anderen einen motorischen Ausgleich für die meist einseitige Belastung zu schaffen. Darüber hinaus konnte Rudolph (2013) bei einer Studie zu einem bewegungsbezogenen Konzept für Forstwirte feststellen, dass die Probanden trotz ihrer hohen beruflichen Aktivität mit einem durchschnittlichen BMI von 29,4 in den übergewichtigen Bereich einzuordnen sind.

Resultierend aus dieser Diskrepanz zwischen hoher körperlicher Aktivität im Beruf und gleichzeitigem Übergewicht bildet die Frage nach der Energiebilanz bei Forstwirten die Grundlage dieser Arbeit. Das Nebeneinander von Übergewicht und hoher körperlicher Beanspruchung, aus der ein hoher Energieumsatz resultiert, lässt eine hohe Energieaufnahme vermuten. Eine positive Energiebilanz könnte das bei der hohen körperlichen Aktivität im Beruf entstandene Übergewicht erklären. Neben der Erhebung des Energieumsatzes bei beruflichen Tätigkeiten ist eine Erhebung des Energieumsatzes in der Freizeit und eine Erhebung der Energieaufnahme notwendig, um die Energiebilanz der Forstwirte betrachten zu können.

Die Erhebungsmethoden basieren auf den Hypothesen, so dass Energieumsatz und Energieaufnahme zu unterschiedlichen Jahreszeiten an allen Wochentagen und bei unterschiedlichen Tätigkeiten im Beruf sowie die Entwicklung der Körperkomposition dargestellt werden konnten. Um die Parameter Energieumsatz, Energieaufnahme, Körperkomposition, Körpermasse, äußere Bedingungen erheben zu können, führten die Probanden zu drei Messzeitpunkten Ernährungs- und Aktivitätsprotokolle und Protokolle über äußere Bedingungen während der Arbeitszeit. Körpermasse und -komposition wurden mittels Waage und Kalipermetrie erhoben. Die indirekte Kalorimetrie zur Erhebung kardiozirkulatorischer Werte wurde während der beruflichen Haupttätigkeiten in den drei Messzeitpunkten zu unterschiedlichen Jahreszeiten (Winter, Frühjahr, Sommer) durchgeführt.

Die Ergebnisse weisen zu allen drei Messzeitpunkten eine negative Energiebilanz auf. Dieses Ergebnis steht im Kontrast zu der Gewichtszunahme und dem ansteigenden Körperfettanteil 
der Probanden. Lediglich am Wochenende konnte eine ausgeglichene Energiebilanz erhoben werden, was ein Indiz dafür ist, dass die Forstwirte am Wochenende trotz der wegfallenden beruflichen Aktivität ihr Essverhalten beibehalten. Es kann daher vermutet werden, dass die gleich bleibende hohe Energiezufuhr am Wochenende als Ausgleich für die negative Energiebilanz der Werktage dient. Zudem wurden die Erhebungen zu Zeitpunkten hochintensiver beruflicher Tätigkeiten durchgeführt. Die Ergebnisse spiegeln somit die negativen Energiebilanzen einzelner Wochen wider, die in angrenzenden Wochen durch beibehaltenes Essverhalten und geringeren Energieumsatz ausgeglichen werden, so dass sich die leichte Gewichtszunahme erklären ließe. Bei der Betrachtung der Energieaufnahme konnte eine Mehraufnahme in t1 im Winter festgestellt werden, die an die erhobenen erhöhten Energieumsätze pro Tag angepasst sind. Grundsätzlich deckt die Energiezufuhr der Forstwirte den Energiebedarf eines Mittelschwerarbeiters, was dem Anforderungsprofil des Berufs Forstwirt entspricht.

Der erhöhte Eiweißanteil bei der Zusammensetzung der Nahrung ist an die körperliche Aktivität der Forstwirte angepasst und ermöglicht den Ersatz und Neuaufbau eiweißhaltiger Substanzen. Bzgl. des Kohlenhydrat- und Fettanteils der Ernährung kann die Zusammensetzung optimiert werden, indem in Anpassung an die hohe körperliche Aktivität der Fettanteil zu Gunsten des Kohlenhydratanteils reduziert wird, um eine optimale Versorgung zu gewährleisten. Die hohe Flüssigkeitszufuhr über meist mineralstoffhaltige Getränke lässt sich auf die hohe körperliche Aktivität zurückführen.

Die Betrachtung des Energieumsatzes, ermittelt aus den Aktivitätsprotokollen, weist den geringsten Energieumsatz im Winter bei Holzernte und den höchsten Energieumsatz im Frühjahr bei Pflanzungen und Sammelhieb auf, obwohl mittels indirekter Kalorimetrie bei der isolierten Messung der beruflichen Tätigkeiten bei der Holzernte der höchste Energieumsatz gemessen wurde. Dieses Phänomen erklärt sich dadurch, dass bei Pflanzungen ein kontinuierliches Arbeiten über längere Zeiträume möglich ist, so dass durch diese Kontinuität ein erhöhter Energieumsatz pro Tag entsteht.

Aufgrund des vermehrten Freizeitanteils am Wochenende ist der Energieumsatz in der Freizeit am Wochenende etwa doppelt so groß wie an Werktagen. Hinsichtlich der sportlichen Aktivität konnte eine höhere Aktivität im Frühjahr festgestellt werden. Einige Forstwirte treiben gar keinen Sport. Generell lässt sich konstatieren, dass sich der Energieumsatz in den Übergangsbereich von schwerer zu schwerster Arbeit einordnen lässt.

Ein Einfluss der Temperatur auf den Energieumsatz konnte nicht festgestellt werden. Im Hinblick auf die Hangneigung und die Behinderung konnte in $\mathrm{t} 2$ ein Zusammenhang zwischen äußeren Bedingungen und Energieumsatz bei Pflanzungen ermittelt werden. Diese Abhängig- 
keit resultiert zum einen aus der kontinuierlichen Fortbewegung am Hang und zum anderen aus der Notwendigkeit, den Bodenbewuchs vor dem Setzen der Pflanzlöcher entfernen zu müssen.

Die Untersuchung mittels indirekter Kalorimetrie bestätigte zunächst, dass die Holzernte den Hauptaufgabenbereich der Forstwirte ausmacht. Neben der Holzernte wurde der Energieumsatz der Forstwirte bei Jungwuchspflege, Pflanzungen, Sammelhieb und Wegebau gemessen. Die hohen Standardabweichungen sind nicht nur auf die geringe Fallzahl, sondern auch auf interpersonale Varianzen zurückzuführen, die sich durch die unterschiedlichen Tätigkeiten, die unterschiedlichen Bedingungen bei der Messung und motorische Stereotype erklären lassen.

Der Energieumsatz der Studie entspricht einem MET zwischen fünf und sieben. Dieser Wert fällt in die Kategorie Schwerstarbeit und geht konform mit dem Anforderungsprofil der Forstwirte. Trotz der Berücksichtigung, dass die Reine Arbeitszeit gemessen wurde, lässt dieser hohe Energieumsatz auf eine zeitliche Begrenzung einer derart hohen Belastung schließen. Somit lässt sich ein Belastungsprofil vermuten, das in verschiedene Phasen eingeteilt ist, welche in ihrer Belastungsintensität variieren. Die Ergebnisse der Fahrradspiroergometrie, anhand derer der Energieumsatz in physikalischen Maßen ausgedrückt werden konnte, unterstreichen die hohe Beanspruchung der Forstwirte.

Bei den Erhebungsinstrumenten handelt es sich um Methoden, die auf die Hypothesen abgestimmt sind. Die Kalipermetrie erwies sich als ausreichend präzise und als gut praktikabel im Feld. Die Erhebung der äußeren Bedingungen während der indirekten Kalorimetrie ermöglichte einen Vergleich der gemessenen Werte unter bestimmten Bedingungen. Die weitere Erhebung der äußeren Bedingungen im Untersuchungszeitraum war nicht zielführend, da sie aufgrund nicht vorhandener Daten zur Auswirkung äußerer Bedingungen auf den Energieumsatz nicht in die Auswertung einfließen konnte. Lediglich eine deskriptive Betrachtung der unterschiedlichen Einflussgrößen war hier möglich.

Die Protokollierung der Ernährung und der Aktivität über sieben Tage erwies sich als zielführend. Durch das gewählte Zeitintervall war ein Vergleich zwischen Werktagen und Wochenende und zwischen beruflicher und Freizeitaktivität möglich. $\mathrm{Zu}$ berücksichtigen bleibt jedoch, dass es sich um Schätzprotokolle handelt und somit um keine konkret gemessenen Daten. Trotz der Tatsache, dass Fehlprotokollierungen stattfinden können und trotz der Abhängigkeit von der Compliance der Probanden, wies diese Methode eine hohe Praktikabilität auf. Dieser positive Aspekt wird durch die in der Literatur argumentierte Exaktheit, die dieser Methode zugeschrieben wird, unterstrichen. Diskutabel ist weiterhin die Methode zur Erhebung 
des Grundumsatzes der Probanden, welcher als Grundlage für das Aktivitätsprotokoll dient. Unterschiedliche Formeln werden in der Literatur diskutiert, so dass sich keine Methode in ihrer Exaktheit von den anderen abhebt. Eine Messung der Grundumsätze scheiterte jedoch bei den Probanden an der Umsetzbarkeit im Feld, so dass die Berechnung des Grundumsatzes anhand der Mifflin-Formel als die realistischste und exakteste eingestuft wurde.

Die indirekte Kalorimetrie als ergänzende Methode zu den Aktivitätsprotokollen erwies sich als sinnvoll. Zum einen konnten die gemessenen Werte in das Aktivitätsprotokoll integriert werden, zum anderen war eine konkrete Evaluation der erhobenen Werte bei den unterschiedlichen Tätigkeiten möglich. Problematisch ist die Tatsache, dass sich die forstwirtschaftlichen Tätigkeiten nicht standardisieren lassen und daher nur bedingt miteinander vergleichbar sind. Die in der Literatur besprochene hohe Praktikabilität im Feld konnte in dieser Studie bestätigt werden, zumal die Forstwirte ohne Einschränkung ihrer Tätigkeit mit angelegter SpirometrieEinheit nachgehen konnten. Die Zuordnung der erhobenen Energieumsätze in bestimmte Leistungsbereiche in Watt, um die erbrachte Leistung physikalisch ausdrücken zu können, hätte im optimalen Fall bei allen Probanden durchgeführt werden können. Dennoch bieten die Werte Anhaltspunkte, in welche Leistungsbereiche in Watt die erbrachte Leistung im Beruf eingeordnet werden kann.

Resümierend lässt sich zusammenfassen, dass für die Probanden der vorliegenden Studie eine fettreduzierte und kohlenhydratangereicherte Ernährung empfohlen werden kann. Durch eine solche Ernährung werden optimale Bedingungen für die Leistungsfähigkeit geschaffen. In Bezug auf die körperliche Aktivität sind eine kohlenhydratreiche Kost vor der Beanspruchung und eine proteinreiche Kost nach der Beanspruchung zu empfehlen. Eine Ernährungsberatung sollte durch Experten erfolgen, um auf individuelle Ernährungsgewohnheiten eingehen zu können. Darüber hinaus ist es trotz der hohen körperlichen Beanspruchung im Beruf dringend ratsam, in der Freizeit körperlich aktiv zu sein. Im physischen als auch im psychosozialen Bereich wird hier ein Ausgleich für die beruflichen Anforderungen geschaffen, so dass im Umkehrschluss durch die erhöhte körperliche Freizeitaktivität die Arbeitsqualität der Forstwirte steigt.

Wünschenswert wäre eine Untersuchung, welche die vermutliche Einteilung der Belastung der Forstwirte in unterschiedliche Phasen mit unterschiedlichen Anforderungsniveaus analysiert. So könnten die Phasen der höheren Belastung definiert werden und es könnte in arbeitsund verfahrenstechnischer Hinsicht nach Möglichkeiten gesucht werden, wie auf diese intervallförmige Belastung reagiert werden kann. Des Weiteren wäre es von großem Interesse, ob bei anderen Berufen mit hoher körperlicher Beanspruchung ähnliche Untergliederungen des 
Anforderungsprofils in unterschiedlich beanspruchende Phasen vorzufinden sind. So könnte ein Präventionskonzept entwickelt werden, um Phasen mit Belastungsspitzen zu vermeiden oder aber im Sinne der Gesunderhaltung die Arbeiter mit Hilfe von Interventionen und Informationsveranstaltungen zu unterstützen. 


\section{Literaturverzeichnis}

Ainsworth, B. E.; Haskell, W. L.; Herrmmann, S. D.; Meckes, N.; Bassett, D. R.; TudorLocke, C.; Greer, J. L.; Vezina, J.; Whitt-Glover, M. C. \& Leon, A. S. (2011). 2011 Compendium of Physical Activities: A Second Update of Codes and MET Values. Medicine \& Science in Sports \& Exercise, 43 (8), 1575-1581.

Ainsworth, B. E.; Haskell, W. L.; Whitt, M. C.; Irwin, M. L.; Swartz, A. M.; Strath, S. J.; O’Brien, W. L.; Bassett, D. R.; Schmitz, K. H.; Emplaincourt, P. O.; Jacobs, D. R. \& Leon, A. S. (2000). Compendium of Physical Activities: an update of activity codes and MET intensities. Medicine \& Science in Sports \& Exercise, 32 (9), 498-504.

Ainsworth, B. E.; Haskell, W. L.; Leon, A. S.; Jacobs, D. R.; Montoye, H. J.; Sallis, J. F. \& Paffenbarger, R. S. (1993). Compendium of Physical Activities: classification of energy costs of human physical activities. Medicine \& Science in Sports \& Exercise, 25 (1), 71-80.

Alvaro, B.; Tang, R.\& Zhu, C. (2011).The effect of mental stress on heart rate variability. New Jerseys Governor`s School of Engineering \& Technology. Zugriff am 22.05.2013 unter: http://soe.rutgers.edu/files/2011HeartRate.pdf.

Arena, R.; Myers, J.; Williams, M. A.; Gulati, M.; Kligfield, P.; Balady, G. J.; Collins, E.\&Fletcher, G. (2007). Assessment of Functional Capacity in Clinical and Research Settings. Journal of the American Heart Association, 116, 329-343.

Åstrand, I. (1987). Arbeitsphysiologie. Stuttgart; New York: Schattauer GmbH.

Atzler, E. (1927). Stoff- und Kraftwechsel. In E. Atzler (Hrsg.), Körper und Arbeit. Handbuch der Arbeitsphysiologie (S. 147-195). Leipzig: Georg Thieme Verlag.

Backhaus, G. (1980). Vorschläge zur methodischen Ermittlung von Rüst-, Verteil- und Erholungszeiten. Forsttechnische Informationen. Mitteilungsblatt des „Kuratorium für Waldarbeit und Forsttechnik“, 32 (81), 1-6.

Baechle, T. R. (Ed.) (1994). Essentials of strength training and conditioning. Champaign, IL: Human Kinetics.

Barreira, T. V.; Kang, M.; Caputo, J. L.; Farley, R. S. \& Renfrow, M. S. (2009). Validation of the Actiheart Monitor for the Measurement of Physical Activity. International of Exercise Science, 2 (1), 60-71.

Bates, G.; Parker, R.; Ashby, L. \& Bentley, T. (2013). Fluid Intake and Hydration Status of Forest Workers - A Preliminary Investigation. International Journal of Forest Engineering, $12(2), 27-32$.

Bellisle, F. (2014). Meals and snacking, diet quality and energy balance. Physiology \& Behavior, 134, 38-43.

Benton, D. (2004). Role of parents in the determination of the food preferences of hildren and the development of obesity. International Journal of Obesity, 28, 858-869. 
Berg, A. \& König, D. (2006). Ernährungsrelevante Lebensstilfaktoren - Sport. In P. Schauder \& G. Ollenschläger (Hrsg.), Ernährungsmedizin. Prävention und Therapie (3., völlig überarbeitete und erweiterte Auflage, S. 407-414). München: Elsevier GmbH.

Biddle, S.\& Mutrie, N. (2008). Psychology of Physical Activity. Determinants, well-being and interventions (2nd edition). New York: Rautledge.

Biesalski, H. K. (2007). Ernährung. InR. F. Schmidt \& F. Lang (Hrsg.), Physiologie des Menschen mit Pathophysiologie (30., neu bearbeitete und aktualisierte Auflage). Heidelberg: Springer Medizin Verlag.

Biolo, G.; Maggi, S. P.; Williams, B. D.; Tipton, K. D. \& Wolfe, R. R. (1995). Increased rates of muscle protein turnover and amino acid transport after resistance exercise in humans. American Journal of Physiology - Endocrinology and Metabolism, 268 (3), 514-520.

Birger, K.; Magnus, H. P. \& Vetlesen, K. (1998). Neck/ shoulder and low-back disorders in the forestry industry: relationship to work tasks and perceived psychosocial job stress. Ergonomics, 41 (10), 1510-1518.

Bitar, A.; Fellmann, N.; Vernet, J.; Coudert, J. \& Vermorel, M. (1999).Variations and determinants of energy expenditure as measured by whole-body indirect calorimetry during puberty and adolescence. American Journal of Clinical Nutrition, 69, 1209-1216.

Boutellier, U. \& Ulmer, H.-V. (2005). Sport- und Arbeitsphysiologie. In R. F. Schmidt; F. Lang \& G. Thews (Hrsg.), Physiologie des Menschen mit Pathophysiologie (29., vollständig neu bearbeitete und aktualisierte Auflage) (909-932). Heidelberg: Springer Medizin.

Bowman, S. A. (2006): Television-Viewing Characteristics of Adults: Correlations to Eating Practices and Overweight and Health Status. Preventing Chronic Disease. Public Health Research, Practice, and Policy, 3 (2), 1-11.

Boyle, M. (2010). Functional Training. Das Erfolgsprogramm der Spitzensportler. München: riva Verlag $\mathrm{GmbH}$.

Brehm, M. A.; Harlaar, J. \& Groepenhof, H. (2004). Validation of the portable VmaxST system for oxygen-uptake measurement. Gait Posture, 20 (1), 67-73.

Burke, L. M.; Kiens, B. \& Ivy, J. L. (2004). Carbohydrates and fat for training and re-covery. Journal of Sports and Sciences, 22, 15-30.

Burt, D. G.; Lamb, G.; Nicholas, C. \& Twist, C. (2013). Effects of exercise-induced muscle damage on resting metabolic rate, sub-maximal running and post-exercise oxygen consumption. European Journal of Sport Science, DOI:10.1080/17461391.2013.783628.

Bundeszentrale für gesundheitliche Aufklärung (2012). Die Drogenaffinität Jugendlicher in der Bundesrepublik Deutschland 2011. Teilband Alkohol. Köln: Bundeszentrale für gesundheitliche Aufklärung.

Cai, H.; Shu, X.; Hebert, J. R.; Jin, F.; Yang, G.; Liu, Y. \& Zheng, W. (2004). Variation in nutrient intakes among women in Shanghai, China. European Journal of Clinical Nutrition, $58,1604-1611$. 
Çalişkan, E. \& Çağlar, S. (2010). An assessment of physiological workload of forest workers in felling operations. African Journal of Biotechnology, 9 (35), 5651-5658.

Clark, D. O.; Lane, K. A.; Ambuehl, R.; Tu, W.; Liu, C.-J. ; Unroe, K. \& Callahan, C. M. (2015). Age Differences in the Association Between Body Mass Index Class and Annualized Medicare Expenditures. Journal of Aging and Health, 1-15. Zugriff am 29.07.2015 unter: http://jah.sagepub.com.9221.han.sub.unigoettingen.de/content/early/2015/06/24/0898264315589574.full.pdf + html

Conrooy, B. P. \& Earle, R. W. (1994). Bone, muscle, and connective tissue adapta-tions to physical activity. In T. R. Baechle (ed.), Essentials of strength training and conditioning (5166). Champaign, IL: Human Kinetics.

De Lorenzo, A.; Bertini, I.; Candeloro, N.; Piccinelli, R.; Innocente, I. \& Brancati, A. (1999). A new predicitve equation to calcolate restino metabolic rate in athletes. Journal of Sports Medicine and Physical Fitness, 39 (3), 213-219.

De Marees, H. (2003). Sportphysiologie (9., vollständig überarbeitete und erweiterte Auflage). Köln: Sportverlag Strauss.

Corral, P. D.; Chandler-Laney, P. C.; Casazza, K.; Gower, B. A. \& Hunter, G. R. (2009). Effect of Dietary Adherence with or without Exercise on Weight Loss: A Mechanistic Approach to a Global Problem. Journal of Clinical Endocrinology \& Metabolism, 94 (5), 1602-1607.

Deutsche Gesellschaft für Ernährung; Österreichische Gesellschaft für Ernährung; Schweizerische Gesellschaft für Ernährungsforschung; Schweizerische Vereinigung für Ernährung (Hrsg.) (2012). Referenzwerte für die Nährstoffzufuhr (1. Auflage, 4. korrigierter Nachdruck). Bonn: Umschau Buchverlag GmbH.

Dickhuth, H.-H. (2010): Internistisch-allgemeinmedizinische Untersuchung. In H.-H. Dickhuth; F. Mayer;K. Röcker \& Berg, A. (Hrsg.), Sportmedizin für Ärzte (2. überarbeitete Auflage, 43-52). Köln: Deutscher Ärzte-Verlag GmbH.

Di Pietro, L.; Dziura, J. \& Blair, S. N. (2004). Estimated change in physical activity level (PAL) and prediction of 5-year weight change in men: the Aerobics Center Longitudinal Study. International Journal of Obesity, 28, 1541-1547.

Dudley, G. A. \&Harris, R. T. (1994). Neuromuscular adaptations to conditioning. In T. R. Baechle (Ed.), Essentials of strength training and conditioning (pp. 12-18).Champaign, IL: Human Kinetics.

Dunford, M. \& Doyle, J. A. (2007). Nutrition for Sport and Exercise ( $2^{\text {nd }}$ Edition). Stamford, Connecticut: Cengage Learning.

Erdogan, A.; Cetin, C.; Karatosun, H. \& Baydar, M. L. (2010). Accuracy of the Polar S810iTM heart rate monitor and the Sensewear Pro ArmbandTM to estimate energy expenditure of indoor rowing exercise in overweight and obese individuals. Journal of Sports Science and Medicine, 9, 508-516. 
Esliger, D. W.; Copeland, J. L.; Barnes, J. D. \& Tremblay, M. S. (2005). Standardizing and Optimizing the Use of Accelerometer Data for Free-Living Physical Activity Monitoring. Journal of Physical Activity and Health, 3, 366-383.

Etheridge, T.; Philp, A. \& Watt, P. W. (2008). A single protein meal increases recovery of muscle function following an acute eccentric exercise bout. Applied Physiology, Nutrition, and Metabolism, 33 (3), 483-488.

FAO/ WHO/ UNU (2001). Human energy requirements. Report of a Joint FAO/ WHO/ UNU Expert Consultation. Zugriff am 29.07.2015 unter: http://www.fao.org/3/a-y5686e.pdf

Faustin, V. \& Ellrott, T. (2006). Praxis der Verzehrsdiagnostik. In P. Schauder \& G. Ollenschläger: Ernährungsmedizin. Prävention und Therapie (3., völlig überarbeitete und erweiterte Auflage, S. 506-518). München: Elsevier GmbH.

Feist, H. (2012). Über die Umsetzung waldbaulicher Ideen. LWF aktuell, 19 (1), 8-10.

Fialka-Moser, V. (2013). Kompendium Physikalische Medizin und Rehabilitation. Diagnostische und therapeutische Konzepte (3. Auflage). Wien: Springer Verlag.

Fleischer, M. (2009): Die Geschichte der Holzernte in Handarbeit (1. Auflage). Halle: Projekt-Verlag Cornelius GmbH.

Frankenfield, D. C.; Rowe, W. A.; Smith, J. S. \& Cooney, R. N. (2003). Validation of several established equations for resting metabolic rate in obese and nonobese people. Journal of the American Dietetic Association, 103 (9), 1152-1159.

Friedrich, O. (2007): Physiologie. Heidelberg: Springer Medizin Verlag.

Gallis, C. \& Panagopoulou, P. (2007). Nutrient intakes of Greek forest workers and researchers do not meet all Reference Dietary Intakes. Nutrition Research, 27 (6), 321-326.

Geliebter, A.; Maher, M. M.; Gerace, L.; Gutin, B.; Heymsfield, S. B. \& Hashim, S. A. (1997). Effects of strength or aerobic training on body composition, resting metabolic rate, and peak oxygen consumption in obese dieting subjects. American Journal of Clinical Nutrition, 66, 557-563.

Gibala, M. J. (2005). Diet For Sport Performance \& Active Lifestyle. Wellness Options, 21, 32-33.

Golenhofen, K. (2006). Basislehrbuch Physiologie. Lehrbuch, Kompendium, Fragen und Antworten (4. überarb. und aktualisierte Auflage). München: Elsevier GmbH.

Graf, C. (2012). Klinische und apparative Diagnostik. In C. Graf (Hg.), Lehrbuch Sportmedizin. Basiswissen, präventive, therapeutische und besondere Aspekte (2., völlig überarbeitete und erweiterte Auflage, S. 43-104). Köln: Deutscher Ärzte-Verlag GmbH.

Graf, C. \& Rost, R. (2012). Körperliche Belastung unter speziellen Bedingungen: Hitze und Kälte. In C. Graf (Hg.), Lehrbuch Sportmedizin. Basiswissen, präventive, therapeutische und besondere Aspekte (2., völlig überarbeitete und erweiterte Auflage, S. 711-722). Köln: Deutscher Ärzte-Verlag GmbH. 
Graf, C. \& Rost, R. (2001). Sportmotorische Hauptbeanspruchungsformen. In: R. Rost (Hrsg.); Lehrbuch der Sportmedizin (S. 41-44). Köln: Deutscher Ärzte-Verlag GmbH.

Graf, C.; Rost, R. \& Wilczkowiak, U. (2001). Sport unter speziellen Bedingungen: Hitze und Kälte. In R. Rost (Hrsg.), Lehrbuch der Sportmedizin (S. 608-617). Köln: Deutscher ÄrzteVerlag GmbH.

Graf, C.; Gottwald, K.; Köhler, R.; Rost, R.; Schänzer, W. (2012). Sporternährung. In C. Graf (Hrsg.), Lehrbuch Sportmedizin. Basiswissen, präventive, therapeutische und besondere Aspekte (2., völlig überarbeitete und erweiterte Auflage, S. 137-196). Köln: Deutscher ÄrzteVerlag GmbH.

Grant, P. M.; Ryan, C. G. \& Tigbe, W.W (2006). The validation of a novel activity monitor in the measurement of posture and motion during everyday activities. British Journal of Sports Medicine, 40, 992-997.

Grunewald, R. W. (2006). Wasser und Mengenelemente. In P. Schauder \& G. Ollenschläger (Hrsg.), Ernährungsmedizin. Prävention und Therapie (3., völlig überarbeitete und erweiterte Auflage, S. 119-133). München: Elsevier GmbH.

Hänsel, F. (2007). Körperliche Aktivität und Gesundheit. In R. Fuchs, W. Göhner \& H. Seelig (Hrsg.), Aufbau eines körperlich-aktiven Lebensstils (S. 23-44). Göttingen: Hogrefe.

Hagen, K. B.; Vik, T.; Myhr, N. E.; Opsahl, P. A. \& Harms-Ringdahl, K. (1993). Physical workload, perceived exertion, and output of cut wood as related to age in motor-manual cutting. Ergonomics, 36 (5), 479-488.

Haines, P. S.; Hama, M. Y.; Guilkey, D. K. \& Popkin, B. M. (2003). Weekend eating in the United States is linked with greater energy, fat and alcohol intake. Obesity Research, 11, 8, 945-949.

Hall, Kevin D. (2010). Predicting metabolic adaptation, body weight change, and energy intake in humans. American Journal of Physiology - Endocrinology and Metabolism, 298, E449-E466.

Harman, E. (1994). The Biomechanics of resistance exercise. In T. R. Baechle (ed.), Essentials of strength training and conditioning (pp. 19-50). Champaign, IL: Human Kinetics.

Hartman, A. M.; Brown, C. C.; Palmgren, J.; Pietinen, P.; Verkasalo, M.; Myer, D. \& Virtamo, J. (1990). Variability in nutrient and food intakes among older middle-aged men: implicaitons for design of epidemiologic and validation studies using food recording. American Journal of Epidemiology, 132 (5), 999-1012.

Heck, H. (1990). Energiestoffwechsel und medizinische Leistungsdiagnostik (8. Studienbrief der Trainerakademie Köln des Deutschen Sportbundes). Schorndorf: Karl Hofmann.

Heilbronn, L. K.; Jonge, L. de; Frisard, M. I.; DeLany, J. P.; Larson-Meyer, D. E. \& Rood, J. et al. (2006). Effect of 6-Month Calorie Restriction on Biomarkers of Longevity Metabolic Adaptation and Oxidative Stress Overweight Individuals. In: Journal of the American Medical Association, 295 (13), 1539-1548. 
Herm, K.-P (2003). Methoden der Körperfettbestimmung. Deutsche Zeitschrift für Sportmedizin, 54 (5), 153-154.

Heseker, H. (2006). Referenzwerte für die tägliche Nahrungszufuhr. In P. Schauder \& G. Ollenschläger (Hrsg.), Ernährungsmedizin. Prävention und Therapie (3., völlig überarbeitete und erweiterte Auflage, S. 41-44). München: Elsevier GmbH.

Hick, C. \& Hick, A. (Hrsg.) (2002). Kurzlehrbuch Physiologie (4. überarbeitete und aktualisierte Auflage). München, Jena: Urban \& Fischer Verlag.

Hollmann, W. \& Hettinger, T. (2000). Sportmedizin. Grundlagen für Arbeit, Training und Präventivmedizin (4., völlig neu bearbeitete und erweiterte Auflage). Stuttgart, New York: Schattauer GmbH.

Hollmann, W.; Strüder, H. K.; Predel, H.-G \& Tagarakis, C. V. M. (2006). Spiroergometrie. Kardiopulmonale Leistungsdiagnostik des Gesunden und Kranken. Stuttgart: Schattauer $\mathrm{GmbH}$.

Hübscher, M. (2011). Sensomotorisches Training. In L. Vogt. (Hrsg.), Sport in der Prävention. Handbuch für Übungsleiter, Sportlehrer, Physiotherapeuten und Trainer (3. vollständig überarbeitete und erweiterte Auflage, S. 84-104). Köln: Deutscher Ärzte-Verlag GmbH.

Huth, W. (2006). Umgang mit der chemischen Energie der Nahrung. In P. Schauder \& G. Ollenschläger (Hrsg.), Ernährungsmedizin. Prävention und Therapie (3., völlig überarbeitete und erweiterte Auflage, S. 57-69). München: Elsevier GmbH.

Ilmarinen, J. (1978): Beziehung zwischen beruflicher und sportlicher körperlicher Aktivität und kardiopulmonaler Leistungsfähigkeit. Dissertation: Deutsche Sporthochschule Köln.

Jacke, H.; Zimmer, F. (2011): Pflanzverfahren im Vergleich. Lasten und Leistungen. Forst \& Technik, 4, 28-31.

Jacke, H. (2013). Mit Stahl am Stiel pflanzen. Wald und Jagd, 17, 46-47.

Jacke, H.; Spiegelhalter, J. \& Hittenbeck, J. (2013). Manuelle Containerpflanzung. Forst \& Technik, 5, 16-21.

Jirikowski, W. (2010). Worauf bei den unterschiedlichen Pflanzverfahren zu achten ist. Zugriff am 19.05.2013 unter: http://www.bauernzeitung.at/?id=2500,79698.

Jones, C.; Routley, V.; Trytell, G.; Ibrahim, J. \& Ozanne-Scmith, J. (2013). A descriptive analysis of work-related fatal injury in older workers in Australia 2000-2009. International Journal of Injury Control and Safety Promotion, 20 (1), 85-90.

Kasper, H. (2000): Ernährungsmedizin und Diätetik (9., neu bearbeitete Auflage). München, Jena: Urban \& Fischer Verlag.

Keytel, L. R.; Goedecke, J. H.; Noakes, T. D.; Hiiloskorpi, H.; Laukkanen, R.; van der Merwe, L. \& Lambert, E. V. (2005). Prediction of energy expenditure from heart rate monitoring during submaximal exercise. Journal of Sports Sciences, 23 (3), 289-297. 
Kieser, W. (2009): Fällen mit Seilwinde leicht gemacht! Forst \& Technik, 5, 18-21.

Koch, B. (2012). Trainingslehre. In C. Graf (Hg.), Lehrbuch Sportmedizin. Basiswissen, präventive, therapeutische und besondere Aspekte (2., völlig überarbeitete und erweiterte Auflage, S. 105-136). Köln: Deutscher Ärzte-Verlag GmbH.

König, D. \& Berg, A. (2010). Ernährung und Substitution. In H.-H. Dickhuth; F. Mayer; K. Röcker \&A. Berg (Hrsg.), Sportmedizin für Ärzte (2. überarbeitete Auflage, S. 479-514). Köln: Deutscher Ärzte-Verlag GmbH.

Koh-Banerjee, P.; Chu, N.; Spiegelman, D.; Rosner, B.; Colditz, G.; Willett, W. \& Rimm, E. (2003). Prospective study of the association of changes in dietary intake, physical activity, alcohol consumption, and smoking with 9-y gain in waist circumference among 16587 US men. American Journal of Clinical Nutrition, 78, 719-727.

Konopka, P. (2009): Sporternährung. Leistungsförderung durch bedarfsangepasste und vollwertige Ernährung (12. Auflage). München: BLV Buchverlag GmbH \& Co. KG (BLV Sportwissen).

Koshimizu, T.; Matsushima, Y.; Yokota, Y.; Yanagisawa, K.; Nagai, S.; Okamura, K.; Komatsu, Y. \& Kawahara, T. (2012). Basal metabolic rate and body composition of elite Japanese male athletes. The Journal of Medical Investigation, 59, 253-260.

Kraut, H. \& Keller, W. (1961). Arbeit und Ernährung. In G. Lehmann (Hrsg.), Arbeitsphysiologie (S. 471-511). Berlin, München, Wien: Urban \& Schwarzenberg.

Kreymann, K. G. (2006). Bestimmung des Energieumsatzes und Energiebedarfs. In P. Schauder \& G. Ollenschläger (Hrsg.), Ernährungsmedizin. Prävention und Therapie (3., völlig überarbeitete und erweiterte Auflage, S. 17-532). München: Elsevier GmbH.

Krink, A. \& Kolschewski, C. (2011). Ernährung, Gesundheit, Sport. In L. Vogt (Hrsg.), Sport in der Prävention. Handbuch für Übungsleiter, Sportlehrer, Physiotherapeuten und Trainer (3. vollständig überarbeitete und erweiterte Auflage, S. 199-226). Köln: Deutscher ÄrzteVerlag GmbH.

Kroidl, R. F.; Schwarz, S. \& Lehnigk, B. (2010). Kursbuch Spiroergometrie. Technik und Befundung verständlich gemacht (2., aktualisierte und erweiterte Auflage). Stuttgart; New York: Georg Thieme Verlag.

Kurumatani, N.; Yamaguchi, B.; Dejima, M.; Enomoto, Y. \& Moriyama, T. (1992). Aerobic capacity of forestry workers and physical demands of forestry operations. European Journal of Applied Physiology, 64, 546-551.

Küster, A. \& Bombosch, F. (1993): Ergonomische Beurteilung neuer Handpflanzverfahren. Allgemeine Forstzeitschrift für Waldwirtschaft und Umweltvorsorge, 25, 128-131.

Kukkonen-Harjula, K. \& Rauramaa, R. (1984). Oxygen consumption of lumberjacks in logging with a power-saw. Ergonomics, 27, 59-65. 
Kuratorium für Waldarbeit und Forsttechnik (2000). Tarifvertrag über die Entlohnung von Holzerntearbeiten. Zugriff am 29.06.2013 unter: http://www.kwfonline.org/fileadmin/dokumente/Mensch_Arbeit/TdL/Lohnentwicklung/EST/est_tvo.pdf.

Kuratorium für Waldarbeit und Forsttechnik (2013). Zuschläge für standorts- und bestandesbezogene Hiebsmerkmale. Zugriff am 29.06.2013 unter: http://www.kwfonli-

ne.org/fileadmin/dokumente/Mensch_Arbeit/TdL/Lohnentwicklung/EST/Anlage_4/tabellea.p df.

Laquale, K. M. (2007). Energy in - energy out: A balanced equation? Athletic Therapy Today, $12(5), 34-37$.

Laube, W. (2009a). Diagnostik der Leistungen des sensomotorischen Systems: Koordination Ausdauer - Kraft. In W. Laube (Hrsg.), Sensomotorisches System. Physiologisches Detailwissen für Physiotherapeuten (S. 228-274). Stuttgart; New York: Georg Thieme Verlag.

Laurent, C. M.; Meyers, M. C.; Robinson, C. A.; Strong, L. R.; Chase, C. \& Goodwin, B. (2008). Validity of the VmaxST portable metabolic measurement system. Journal of Sports Sciences, 26 (7), 709-716.

Lehmann, G. (1961a): Das physische Leistungsvermögen des Menschen. In G. Lehmann (Hrsg.), Arbeitsphysiologie (S. 320-361). Berlin, München, Wien: Urban \& Schwarzenberg.

Lehmann, G. (1961b). Die Verbesserung des Leistungsvermögens. In G. Lehmann (Hrsg.), Arbeitsphysiologie (S. 362-404). Berlin, München, Wien: Urban \& Schwarzenberg.

Lehmann, G. (1961c). Energetik des arbeitenden Menschen. In G. Lehmann (Hrsg.), Arbeitsphysiologie (S. 66-121). Berlin, München, Wien: Urban \& Schwarzenberg.

Lehmann, G. \& Schmidtke, H. (1961). Probleme der Lohnfindung. In G. Lehmann (Hrsg.), Arbeitsphysiologie (S. 857-894). Berlin, München, Wien: Urban \& Schwarzenberg.

Lemon, P. W. R. (1991). Effect of exercise on protein requirements. Journal of Sports Sciences, 9, 53-70.

Lemon, P. W. R. (1998). Effects of Exercise of Dietary Protein Requirements. In: International Journal of Sport Nutrition, 8, 426-447.

Levine, J. A.; Lanningham-Foster, L. M.; McCrady, S. K.; Krizan, A. C.; Olson, L. R.; Kane, P. H. et al. (2005). Interindividual Variation in Posture Allocation: Possible Role in Human Obesity. Science, 307, 584-586.

Levine, J. A. \& Miller, J. M. (2007). The energy expenditure of using a "walk-and-work" desk for office workers with obesity. In: British Journal of Sports Medicine, 41, 558-561.

Liebman, M.; Pelican, S.; Moore, S. A.; Holmes, B.; Wardlaw, M. K.; Melcher, L. M. et al. (2003). Dietary intake, eating behaviour, and physical activity-related determinants of high body mass index in rural communities in Wyoming, Montana, and Idaho. International Journal of Obesity, 27, 684-92. 
Lilley, R.; Feyer, A.-M.; Kirk, P. \& Gander, P. (2002). A survey of forest workers in New Zealand: Do hours of work, rest, and recovery play a role in accidents and injury? Journal of Safety Research, 33 (1), 3-71.

Lippke, S.\& Vögele, C. (2006). Sport und körperliche Aktivität. In B. Renneberg \& P. Hammelstein (Hrsg.), Gesundheitspsychologie (S. 195-216). Heidelberg: Springer.

Livingstone, M. B. E. (1997). Heart-rate monitoring: the answer for assessing energy expenditure and physical activity in population studies? British Journal of Nutrition, 78, 869871.

LWF (2004). Holzernte in steilen Hanglagen. Zugriff am 19.05.2013 unter: http://www.lwf.bayern.de/veroeffentlichungen/lwf-merkblaetter/mb-13-holzerntehanglagen.pdf.

Ma, Y.; Olendzki, B.; Chiriboga, D.; Hebert, J. R.; Li, Y.; Li, W. et al. (2005). Association between Dietary Carbohydrates and Body Weight. American Journal of Epidemiology, 161 (4), 359-367.

Ma, Y.; Olendzki, B. C.; Li, W.; Hafner, A. R.; Chiriboga, D.; Hebert, J. R. et al. (2006). Seasonal variation in food intake, physical activity, and body weight in a predominantly overweight population. European Journal of Clinical Nutrition, 60, 519-528.

Maagh, P. (2006). Determinanten des Sauerstoffverbrauchs in Allgemeinanästhesie : ein Vergleich von Körperoberfläche und Körperzellmasse. Dissertation: Universität Bochum. Zugriff am 22.05.2012 unter: http://deposit.d-nb.de/cgi-bin/dokserv?idn=981390013.

Macfarlane, D. J. \& Wong, P. (2012). Validity, reliability and stability of the portable Cortex Metamax 3B gas analysis system. European Journal of Applied Physiology, 112 (7), 25392547.

Manore, M. M. (2004). Nutrition and Physical Activity: Fueling the Active Individual. President's Council on Physical Fitness and Sports Research Digest, 5 (1), 1-8.

Manore, M.; Meyer, N. L. \& Thompson, J. (2009). Sport Nutrition for Health and Performance (2. Auflage). Champaign IL: Human Kinetics.

Matthews, C. E.; Chen, K. Y.; Freedson, P. S.; Buchowski, M. S.; Beech, B. M.; Pate, R. R. \& Troiano, R. P. (2008). Amount of Time Spent in Sedentary Behaviors in the United States, 2003-2004. American Journal of Epidemiology, 167 (7), 875-881.

McArdle, W. D.; Katch, F. I. \& Katch, V. L. (2000). Essentials of Exercise Physiology (2nd Edition). Philadelphia: Lippincott Williams \& Wilkins.

McCarthy, S. (2014). Weekly patterns, diet quality and energy balance. Physiology \& Behavior, 134, 55-59.

Melanson, E. L.; Gozansky, W. S.; Barry, D. W.; Maclean, P. S.; Grunwald, G. K. \& Hill, J. O. (2009). When energy balance is maintained, exercise does not induce negative fat balance in lean sedentary, obese sedentary, or lean endurance-trained individuals. Journal of Applied Physiology, 107 (6), 1847-1856. 
Mellerowicz, H. (1979). Ergometrie. Grundriß der medizinischen Leistungsmessung (3. überarbeitete und erweiterte Auflage). München, Wien; Baltimore: Urban \& Schwarzenberg.

Meyer, T.; Davison, R. C. \& Kindermann, W. (2005). Ambulatory gas exchange measurements - current status and future options. International Journal of Sports Medicine, 26 (1), $19-27$.

Mifflin, M. D.; St. Jeor, S. T.; Hill, L. A.; Scott, B. J.; Daugherty, S. A. \& Koh, Y. O. (1990). A new predictive equation for resting energy expenditure in healthy individuals. American Journal of Clinical Nutrition, 51 (2), 241-247.

Ministerium Ländlicher Raum Baden-Württemberg (1997). Richtlinien zur Jungbestandspflege. Stuttgart: Ministerium ländlicher Raum Baden-Württemberg.

Ministerium Ländlicher Raum Baden-Württemberg (2007). Arbeitssicherheit auf den Punkt gebracht. Waldarbeit: Staatsforstbetrieb.

Montgomery, P.; Green, D. J.; Etxebarria, N.; Pyne, D. B.; Saunders, P. U. \& Minahan, C. L. (2009). Validation of heart rate monitor-based predictions of oxygen uptake and energy expenditure. Journal of Strength \& Conditioning Research, 23 (5), 1489-1495.

Montoye, H. J.; Kemper, H. D. G.; Saris, W. H. M. \& Washburn, R. A. (1996). Measuring Physical Activity and Energy Expenditure. Champaign IL: Human Kinetics.

Nelson, Miriam E.; Rejeski, W. Jack; Blair, Steven N.; Duncan, Pamela W.; Judge, James O.; King, Abby C. et al. (2007). Physical Activity and Public Health in Older Adults. Recommendation From the American College of Sports Medicine and the American Heart Association. Circulation, 116, 1094-1105.

Niermann, C. (2011). Vom Wollen und Handeln. Selbststeuerung, sportliche Aktivität und gesundheitsrelevantes Verhalten. (Schriften der Deutschen Vereinigung für Sportwissenschaft, 209). Hamburg: Feldhaus Verlag GmbH \& Co. KG.

Nieß, A. (2010). Thermoregulation und körperliche Aktivität. In H.-H. Dickhuth; F. Mayer; K. Röcker \& A. Berg (Hrsg.), Sportmedizin für Ärzte (2. überarbeitete Auflage, S. 529-538). Köln: Deutscher Ärzte-Verlag GmbH.

Nowak, D. (2003). Arbeitsmedizin. Zum Lernzielkatalog nach der neuen Approbationsordnung (1. Auflage). München, Jena: Elsevier GmbH.

Oberriter, H. (2006). Prinzipien vollwertiger Ernährung. In P. Schauder \& G. Ollenschläger (Hrsg.), Ernährungsmedizin. Prävention und Therapie (3., völlig überarbeitete und erweiterte Auflage, S. 201-220). München: Elsevier GmbH.

O'Dwyer, N. J.; Neilson, P. D. (2000): Metabolic Energy Expenditure and Accuracy in Movement: Relation to Levels of Muscle and Cardiorespiratory Activation and the Sense of Effort. In W. A. Sparrow (Ed.), Energetics of Human Activity (pp. 1-42).Champaign, Il: Human Kinetics. 
Olivier, N. \& Rockmann, U. (2003). Grundlagen der Bewegungswissenschaft und -lehre . Grundlagen der Sportmedizin. Schorndorf: Verlag Karl Hofmann.

Payne, P. R.; Wheeler, E. F. \& Salvosa, C. B. (1971). Prediction of daily energy expenditure from average pulse rate. American Journal of Clinical Nutrition, 24, 1164-1170.

Perkins, C. D.; Pivarnik, J. M. \& Green M. R. (2004). Reliability and validity of the VmaxST portable metabolic analyzer. Journal of Physical Activity end Health, 1 (4), 413-422.

Persson, P. B. (2007). Energie- und Wärmehaushalt, Thermoregulation. In R. F. Schmidt \& F. Lang (Hrsg.), Physiologie des Menschen mit Pathophysiologie (30., neu bearbeitete und aktualisierte Auflage). Heidelberg: Springer Medizin Verlag.

Peterson, K. E.; Dubowitz, T.; Stoddard, A. M.; Troped, P. J.; Sorensen, G. \& Emmons, K. M. (2007). Social Context of Physical Activity and Weight Status in Working-Class Populations. Journal of Physical Activity and Health, 4, 381-396.

Pettitt, R. W.; Pettitt, C. D.; Cabrera, C. A.; Murray, S. R. (2007). A Theoretical Method of Using Heart Rate to Estimate Energy Expenditure During Exercise. International Journal of Sports Science \& Coaching, 2 (3), 319-327.

Pietrowsky, R.(2006). Ernährung. In: B. Renneberg \& P. Hammelstein (Hrsg.), Gesundheitspsychologie, Heidelberg: Springer, S. 173-194.

Prieur, F.; Castells, J. \& Denis, C. (2003). A methodology to assess the accuracy of a portable metabolic system (VmaxST). Medicine \& Science in Sports \& Exercise, 35 (5), 879-885.

Pronk, N. P.; Martinson, B.; Kessler, R. C.; Beck, A. L.; Simon, G. E. \& Wang P. (2004). The Association Between Wark Performance and Physical Activity, Cardiorespiratory Fitness, and Obesity. Journal of Occupational \& Environmental Medicine, 46, 19-25.

Pudel, V. \& Westenhöfer, J. (2003). Ernährungspsychologie - Eine Einführung (3., unveränderte Auflage). Göttingen: Hogrefe.

Pfeiffer, A. F. H. (2006). Regulation des Essverhaltens. In P. Schauder \& G. Ollenschläger (Hrsg.), Ernährungsmedizin. Prävention und Therapie (3., völlig überarbeitete und erweiterte Auflage, S. 45-50). München: Elsevier GmbH.

Platen, P. (2012). Energiebereitstellung. In C. Graf (Hrsg.), Lehrbuch Sportmedizin. Basiswissen, präventive, therapeutische und besondere Aspekte (2., völlig überarbeitete und erweiterte Auflage, S. 19-42). Köln: Deutscher Ärzte-Verlag GmbH.

Platen, P. (2001). Beurteilung der körperlichen Leistungsfähigkeit. In R. Rost (Hrsg.), Lehr buch der Sportmedizin (S. 48-66). Köln: Deutscher Ärzte-Verlag GmbH.

Raab, R. (Hrsg.) (1981). Jungbestandspflege und Wertastung. München: Bayerisches Staatsministerium für Ernährung, Landwirtschaft und Forsten.

Raab, S.; Feller, S.; Uhl, E.; Schäfer, A.; Ohrner, G. (2002). Aktuelle Holzernteverfahren am Hang. Freising: Bayerische Landesanstalt für Wald und Forstwirtschaft (LWF). 
Redding, E.; Wyon, M.; Shearman, J. \& Doggart, L. (2004). Validity of Using Heart Rate as a Predictor of Oxygen Consumption in Dance. Journal of Dance Medicine \& Science, 8 (3), 6972.

REFA-Fachausschuss Forstwirtschaft (1991). Anleitung für forstliche Arbeitsstudien: Datenermittlung, Arbeitsgestaltung (3. Auflage). Darmstadt: REFA.

REFA-Fachausschuss Forstwirtschaft (Hrsg.). (2004). Organisation in der Forstwirtschaft: mit REFA-Methoden zu effizienten Arbeitsprozessen (2. überarbeitete Auflage). Darmstadt: Verband für Arbeitsgestaltung, Betriebsorganisation und Unternehmensentwicklung.

REFA-Verband für Arbeitsstudien und Betriebsorganisation (1987). Methodenlehre der Betriebsorganisation: Anforderungsermittlung (Arbeitsbewertung). München: Hanser.

REFA- Verband für Arbeitsstudien und Betriebsorganisation (1991). Methodenlehre der Betriebsorganisation: Grundlagen der Arbeitsgestaltung. München: Hanser.

Röcker, K. (2010): Leistungsdiagnostische Verfahren. In H.-H. Dickhuth; F. Mayer; K. Röcker \& A. Berg (Hrsg.), Sportmedizin für Ärzte (2. überarbeitete Auflage; S. 65-72). Köln: Deutscher Ärzte-Verlag GmbH.

Rost, R. (2001). Sporternährung. In R. Rost (Hrsg.), Lehrbuch der Sportmedizin (S. 84-133). Köln: Deutscher Ärzte-Verlag GmbH.

Rost, R. \& Hollmann, W. (1982). Belastungsuntersuchungen in der Praxis. Grundlagen, Technik und Interpretation ergometrischer Untersuchungsverfahren. Georg Thieme Verlag: Stuttgart, New York.

Roth, K. \& Willimczik, K. (1999). Bewegungswissenschaft. Reinbek: Rowohlt Taschenbuch Verlag GmbH.

Rothkegel, W.; Ruppert, O. \& Peter, J. (2012). Jungbestandspflege - wichtige Weichenstellung für zukünftige Wälder. LWF aktuell, 19, 1, 4-7.

Rudolph, S. (2013). „Fit im Forst“ - Eine bewegungsbezogene Intervention für Forstwirte. Dissertation, Georg-August Universität Göttingen: Universitätsverlag Göttingen.

Santos, M. P.; Matos, M. \& Mota, J. (2005). Seasonal Variations in Portuguese Adolescents` Organized and Nonorganized Physical Activities. Pediatric Exercise Science, 17, 390-398.

Saris, W. H. M.; Blair, S. N.; van Baak, M. A.; Eaton, S. B.; Davies, P. S. W.; Di Pietro, L. et al. (2003). How much physical activity is enough to prevent unhealthy weight gain? Outcome of the IASO 1st Stock Conference and consensus statement. International Association for the Study of Obesity, 4, 101-114.

Scharhag-Rosenberger, F. \& Schommer, K. (2013). Die Spiroergometrie in der Sportmedizin. Deutsche Zeitschrift für Sportmedizin, 64, 362-366.

Schauder, P. (2006). Makronährstoffe und Ballaststoffe. In P. Schauder \& G. Ollenschläger (Hrsg.), Ernährungsmedizin. Prävention und Therapie (3., völlig überarbeitete und erweiterte Auflage, S. 84-94). München: Elsevier GmbH. 
Schauder, P. \& Arends, J. (2006). Erfassung und Beurteilung des Ernährungszustands. In P. Schauder \& G. Ollenschläger (Hrsg.), Ernährungsmedizin. Prävention und Therapie (3., völlig überarbeitete und erweiterte Auflage, S. 477-4997). München: Elsevier GmbH.

Schauder, P. \& Ollenschläger, G. (Hg.) (2006). Ernährungsmedizin. Prävention und Therapie (3., völlig überarbeitete und erweiterte Auflage). München: Elsevier GmbH.

Scherer, H.-G (1996). Normierte Arbeitsablaufabschnitte als Orientierungshilfen für die Leistungsgradbeurteilung. Dissertation, Georg-August Universität Göttingen: Cuvillier Verlag.

Schlicht, W. \& Brand, R. (2007). Körperliche Aktivität, Sport und Gesundheit. Eine interdisziplinäre Einführung. Weinheim: Juventa.

Schneider, R. \& Heseker, H. (2006). Erfassung von Ernährungsgewohnheiten. In P. Schauder \& G. Ollenschläger (Hrsg.), Ernährungsmedizin. Prävention und Therapie (3., völlig überarbeitete und erweiterte Auflage, S. 498-505). München: Elsevier GmbH.

Schommer, K. \&Bärtsch, P. (2012). Körperliche Belastung unter speziellen Bedingungen: Höhe. In C. Graf (Hrsg.), Lehrbuch Sportmedizin. Basiswissen, präventive, therapeutische und besondere Aspekte (2., völlig überarbeitete und erweiterte Auflage, S. 695-707). Köln: Deutscher Ärzte-Verlag GmbH.

Schusdziarra, V. (2003). Adipositas. Moderne Konzepte für ein Langzeitproblem (2. Auflage). Bremen: UNI-MED Verlag AG.

Schutz, Y.; Weinsier, R. L.\& Hunter, G. R. (2001).Assessment of Free-Living Physical Activity in Humans: An Overview of Currently Available and Proposed New Measures. Obesity Research, 9 (6), 368-379.

Schwarzer, R. (2004). Psychologie des Gesundheitsverhaltens. Einführung in die Gesundheitspsychologie (3. überarbeitete Auflage). Göttingen: Hogrefe.

Semenick, D. M. (1994 a). Selecting appropriate Tests. In T. R. Baechle (Ed.), Essentials of strength training and conditioning (pp. 250-253).Champaign, IL: Human Kinetics.

Semenick, D. M. (1994 b). Testing protocols and procedures. In T. R. Baechle (Ed.), Essentials of strength training and conditioning (pp. 258-273).Champaign, IL: Human Kinetics.

Shephard, R. J. \& Aoyagi, Y. (2009). Seasonal variations in physical activity and implications for human health. European Journal of Applied Physiology, 107, 251-271.

Silva, P.; Santos, R.; Welk, G. \& Mota, J. (2011). Seasonal differences in physical activity and sedentary patterns: The relevance of the PA context. Journal of Sports Science and Medicine, 10, 66-72.

Sluik, D.; de Graaf, K.; de Groot, L. \& Cavelaars, A. (2008). Recommendations for nutrient composition of combat rations for the NATO Response Force. Zugriff am 29.07.2015 unter: http://ftp.rta.nato.int/public/PubFullText/RTO/TR/RTO-TR-HFM-154/TR-HFM-154-ANNJ.pdf 
Sohns, H. (2012): Moderne Holzernte. Stuttgart: Eugen Ulmer KG.

Sparrow, W. A.; Hughes, K. M.; Russell, A. P. \&Le Rossignol, P. F. (2000). Movement Economy, Preferred Modes, and Pacing. In W. A. Sparrow (Ed.), Energetics of Human Activity, (pp. 96-123).Champaign IL: Human Kinetics.

Spendlove, J.; Mitchell, L.; Gifford, J.; Hackett, D.; Slater, G.; Cobley, S. \& O’Connor, H. (2015). Dietary Intake of Competitive Bodybuilders. Sports Medicine, 45 (7), 1041-1063.

Spiegelhalter, J. (2012). Abschlussbericht zum Projekt: Ergonomie und Produktivität bei der Containerpflanzung.

Spierer, D. K. \& Hagins, M. (2011). A comparison of energy expenditure estimates from the Actiheart and Actical physical activity monitors during low intensity activities, walking, and jogging. European Journal of Applied Physiology, 111, 659-667.

Spurr, G. B.; Prentice, A. M.; Murgatroyd, P. R.; Goldberg, G. R.; Reina, J. C. \& Christman, N. T. (1988). Energy expenditure from minute-by-minute heart-rate recording: comparison with indirect calorimetry. American Journal of Clinical Nutrition, 48, 552-559.

Stensel, D. (2008). Obesity and diabetes. In J. Buckley (Ed.), Exercise Physiology on Special Populations (Advances in Sport and Exercise Science series, pp. 21-49). Edinburgh: Churchill Livingstone.

Stihl, N. (1997): Untersuchungen zur komplexen Optimierung des Arbeitssystems "motormanuelle Waldarbeit". Chemnitz: Institut für Betriebswissenschaften und Fabriksysteme.

Stone, M. H. (1994a): Nutritional factors in performance and health. In T. R. Baechle (Ed.), Essentials of strength training and conditioning (pp. 210-230). Champaign, IL: Human Kinetics.

Stone, M. H. (1994b). Weight gain and weight loss. In T. R. Baechle (Ed.), Essentials of strength training and conditioning (pp. 231-237).Champaign, IL: Human Kinetics.

Straker, L. \& Mathiassen, S. E. (2009). Increased physical work loads in modern work - a necessity for better health and performance? Ergonomics, 52 (10), 1215-1225.

Sullman, M. J. H. \& Byers, J. (2000). An Ergonomic Assessment of Manual Planting Pinus Radiata Seedling. Journal of Forest Engineering, 11 (1), 53-62.

Thiel, C. (2011). Bewegung zur Prävention chronischer Erkrankungen. In L. Vogt (Hrsg.), Sport in der Prävention. Handbuch für Übungsleiter, Sportlehrer, Physiotherapeuten und Trainer (3. vollständig überarbeitete und erweiterte Auflage, S. 35-50). Köln: Deutscher Ärzte-Verlag GmbH.

Thompson, J. \& Manore, M. (1996). Predicted and measured resting metabolic rate of male and female endurance athletes. Journal of American Diet Association, 96 (1), 30-34. 
Thompson, B. J.; Ryan, E. D.; Sobolewski, E. J. \& Smith-Ryan, A. E. (2015). Dietary protein intake is associated with maximal and explosive strength of the leg flexors in young and older blue collar workers. Nutrition Research, 35 (4), 280-286.

Tipton, K. D. \& Wolfe, R. R. (2004). Protein and amino acids for athletes. Journal of Sports and Sciences, 22, 65-79.

Tittel, K. \& Wutscherk, H. (1972). Sportanthropometrie: Aufgaben, Bedeutung, Methodik und Ergebnisse biotypologischer Erhebungen. Leipzig: Barth.

Tommlinson, R. W. \& Manenica, I. (1977). A study of physiological and work study indices of forestry work. Applied Ergonomics, 8 (3), 165-172.

Tremblay, A. \& Chaput, J. P. (2008). About unsuspected potential determinants of obesity. Applied Physiology, Nutrition, and Metabolism, 33, 791-796.

Tremblay, A. \& Therrien, F. (2006). Physical activity and body functionality: implications for obesity prevention and treatment. Canadian Journal Physiological Pharmacology, 84, 149156.

Tucker, P. \& Gilliland, J. (2007). The effect of season and weather on physical activity: A systematic review. Public Health, 121, 909-922.

Vaclavik, V. A. \& Christian, E. W. (2008). Essentials of Food Science (3. ed.). New York: Springer.

Van Hees, V.; Gorzelniak, L.; Dean León, E. C.; Eder, M.; Pias, M. et al. (2013). Separating Movement and Gravity Components in an Acceleration Signal and Implications for the Assessment of Human Daily Physical Activity. PLOS ONE, 8 (4), e61691. Zugriff am 21.05.2013 unter: http://www.ncbi.nlm.nih.gov/pmc/articles/PMC3634007/.

Versuchs- und Lehrbetrieb für Waldarbeit und Forsttechnik beim Hessischen Forstamt Diemelstadt (Hrsg.) (1996). Aktuelle Pflanzverfahren: Handpflanzung und motormanuelle Pflanzung. Groß-Umstadt: KWF.

Vogl, R. (2004). Der Einfluss der Atemmasken auf die Herzfrequenz bei Fahrradergometrie und die mögliche Konsequenz auf berechnete Leistungsparameter. Dissertation: Universität Frankfurt am Main.

Vogler, A. J.; Rice, A. J. \& Gore, C. J. (2010). Validity and reliability of the Cortex MetaMax3B portable metabolic system. Journal of Sports Sciences, 28 (7), 733-742.

Vogt, L. \& Töpper, A. (Hrsg.) (2011). Sport in der Prävention. Handbuch für Übungsleiter, Sportlehrer, Physiotherapeuten und Trainer (3. vollständig überarbeitete und erweiterte Auflage). Köln: Deutscher Ärzte-Verlag GmbH.

Wästerlund, D. S. (1998). A review of heat stress research with application to for-estry. Applied Ergonomics, 29, 3, 179-183. 
Wagner, P.\& Brehm, W. (2008). Körperlich-sportliche Aktivität und Gesundheit. In J. Beckmann \& M. Kellmann (Hrsg.), Enzyklopädie der Psychologie. Serie V. Anwendungen der Sportpsychologie (S. 543-608). Göttingen: Hogrefe.

Wareham, N. (2007). Physical activity and obesity prevention. Obesity Reviews, 8, 1, 109114.

Wenzel, H. G. (1961): Die Wirkung des Klimas auf den arbeitenden Menschen. In G. Lehmann (Hrsg.), Arbeitsphysiologie. Berlin, München, Wien: Urban \& Schwarzenberg.

Wenzel, P. \& Stratmann, J. (2006). Technik bei der Jungwuchs- und Jungbestands-pflege. Groß-Umstadt: Kuratorium für Waldarbeit und Forsttechnik.

Westerterp, K. R.; Plasqui, G. \& Goris, A. H. C. (2005). Water loss as a function of energy intake, physical activity and season. British Journal of Nutrition, 93, 199-203.

Winkler, G.; Döring, A. \& Keil, U. (1991). Unterschiede im Ernährungsverhalten zwischen Wochenenden und Werktagen: Ergebnisse der Ernährungserhebung 1984/85 der MONICAProjektes Augsburg. Zeitschrift für Ernährungswissenschaft, 30, 313-317.

Winterhoff, B. (1991). Einfluß des Alterns auf die Leistung der Waldarbeiter. Dissertation: Georg-August Universität Göttingen.

Wirth, A. (2003). Adipositas-Fibel (2., vollständig überarbeitete Auflage). Berlin: Springer.

Wirth, A. (2008). Therapie. In A. Wirth (Hrsg.), Adipositas: Ätiologie, Folgekrankheiten, Diagnose, Therapie; mit 60 Tabellen (3., vollständig überarbeitete und erweiterte Auflage, S. 263-372). Heidelberg: Springer Medizin.

Wirtschafts- und Forstverlag Euting (Hrsg.) (1996). Arbeitsschutz in der Forstwirtschaft - ein dunkles Kapitel. Die Waldarbeit, 47 (12), 163.

World Health Organisation (2014). Global status report on alcohol and health 2014. Luxembourg: World Health Organisation.

Wuorinen, E. C. (2014). The Psychophysical Connection Between Exercise, Hunger, and Energy Intake. American Journal of Lifestyle \& Medicine, 8 (3), 159-163.

Yoshimura, T. \& Acar, H. H. (2004). Occupational safety and health conditions of forestry workers in Turkey. Journal of Forest Research, 9 (3), 225-232.

Zalpour, C. (Hrsg.) (2002). Anatomie. Physiologie (1. Auflage). München, Jena: Urban \& Fischer Verlag.

Zhang, K.; Werner, P.; Sun, M.; Pi-Sunyer, F. X. \& Boozer, C. (2003). Measurement of Human Daily Physical Activity. Obesity Research, 11 (1), 33-40.

Zimmer, Florian (2010). Vergleichende Arbeitszeitstudie mit ergänzender Ergospirometrie zwischen dem Neheimer und Rhodener Pflanzverfahren sowie der Winkelpflanzung. Masterarbeit: Georg-August Universität Göttingen. 


\section{Eidesstattliche Erklärung:}

„Ich versichere, dass ich die eingereichte Dissertation „Energiebilanz bei Forstwirten Diskrepanz zwischen Energieumsatz und Nährstoffaufnahme bei unterschiedlichen Anforderungssituationen" selbstständig und ohne unerlaubte Hilfsmittel verfasst habe. Anderer als der von mir angegebenen Hilfsmittel und Schriften habe ich mich nicht bedient. Alle wörtlich oder sinngemäß den Schriften anderer Autoren entnommenen Stellen habe ich kenntlich gemacht."

Göttingen, den 20.08.2015

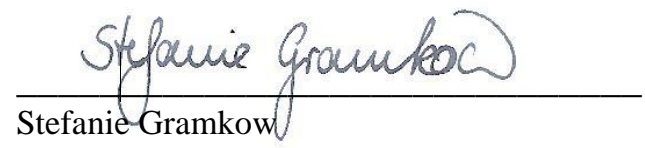

